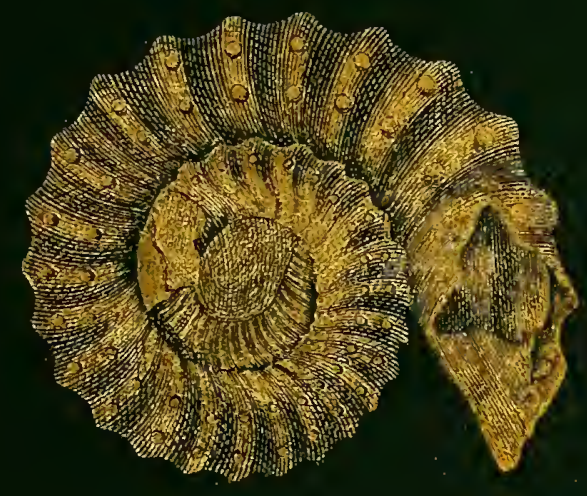





R. S. Bassler.

\title{
PALAONTOLOGY OF CALIFORNIA.
}

\author{
V O L. I I.
}





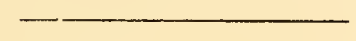

\title{
P A L A O TOLOGY.
}

\author{
V O L U ME II.
}

CRETACEOUS AND TERTIARY FOSSILS.

BY W. M. GABB.

PUBLISHED BY AUTHORITY OF THE LEGISLATURE OF CALIFORNIA.

1869. 



\section{TABLE OF CONTENTS.}

Preface (bx J. D. Whithey), • • • • • • • • vii-xiv

SECTION T.

Part. I.-Description of the Tertiart Invertebrate Fossils, . 1-38

II.-Tertiarx Invertebrate Fossils-Continued, . . . 39-63

III.-Synopsis of the Tertiary Inyertebrate Fossils of Cal-

IFORNIA, • • • • • • • • . . 67-124

\section{SECTION II.}

Part I.-Description of New, and Reyision of Prefiodsly DeScribed, Cretaceous Fossils, . . • • . . . 127-205

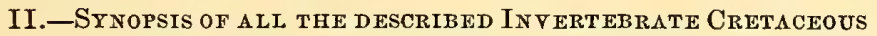

Fossils of California and the Adjacent States, . 209-254

\section{SECTION III.}

Description of Cretaceous Fossils, collected b A. Remond, at Arivechi, Sonora, Mexico, . . . . . . . 257-276

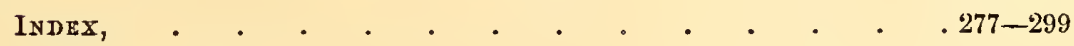





\section{P R E F A C E.}

Volume I of the Palæontology of California was issued in 1864, the preface bearing date September of that year. It contained descriptions of the Carboniferous and Jurassic fossils by Mr. Meek, and of the Triassic and Cretaceous by Mr. Gabb, and is illustrated by thirty-two plates. The present volume is devoted to the Tertiary and Cretaceous palæontology, and is entirely the work of Mr. Gabb. At the time the first volume of the Palæontology was published-which was also the first of our Report-it was thought that the whole of our material in this department of the Survey would be comprised in two volumes. Events have proved, however, as might perhaps have been foreseen, that at least three volumes will. be required for the proper exposition of our palæontological results. The Cretaceous and Tertiary invertebrate material alone requires a volume by itself, and for a third, and possibly a fourth, we have still such additions as have been made to the Jurassic and Triassic, as well as all the fossil plants of various ages, and the vertebrate remains, chiefly of freshwater and land animals, and of Tertiary age; besides these, are the microscopic fossil organisms, both marine and freshwater, which are interesting, and will require considerable space for their illustration.

In the preface of Volume I of the Palæontology, a brief ${ }^{\text {. }}$ 
synopsis of the field-work of the Survey was given, up to the close of the year 1863. In Gcology Vol. I, a synopsis of the operations of the Survey was given up to the datc of November, 1865, which is that of the prefacc of the volume. For the convenience of those who may possess the palæontological volumes of the Report only, the résumć of our work given in Volume I of that series, will here be continued, in as concise a manner as possible, up to the date of the suspension of the field-work in 1867, the failure of the Legislature of 1867-68 to makc an appropriation for the continuance of the Survey, having compelled us to bring our active operations to a close. The question of the resumption of the field-work is one to be decided by a future Legislature.

The party under the dircetion of Professor Brewer, mentioned in the Prefice of the Palæontology, Vol. I, as being in the field at the time of its publication, and which commenced operations in May, proceeded across the plains of the San Joaquin to Visalia, from which point they cntered the Sicrra, ascending King's River to its source, and cxploring the whole region about the hcadwaters of that and Kcrn River. Thence thcy made their way across the rangc by a pass over 12,000 feet high; passed up Owen's Valley, ascended the west branch of Owen's River, crossing the Sicrra again at an altitude of 12,400 feet, and thence descending to the head of the San Joaquin River. The exploration was continued through the region of the headwaters of that stream and the Merced, connecting the reconnoissance with that of 1863 around the sources of the Tuolumne. The wholc expedition occupied about thrce months, during which time the gcography and gcology of a district including an area of over 10,000 square milcs were, for the first time, cxplored, the wholc region having previously been entirely unknown. The results 
proved to be of the greatest interest, disclosing the fact that this was the highest part of the Sierra Nevada, and that it embraced the loftiest mountains and the grandest scenery yet discovered within the territory of the United States. For the details of this reconnoissance, reference may be made to Chapter X of Volume I of the Geology.

Mr. Gabb was in the field in Northern California, Southern Oregon and Idaho, from June 1st, 1864, to about the middle of October. During that expedition he obtained information and specimens proving the existence of the Cretaceous formation on Crooked River-a branch of the Des Chutes-the first discovery of rocks of this age anywhere to the east of the Cascade Range, as noticed in this volume, page 181 .

Very little field-work was done during this year, or the next (in 1865), in regions where fossiliferous rocks occur. Mr. Rémond, during his explorations between the Merced and Stanislaus Rivers, in 1865, made for the purpose of working up the detailed geology of that region, discovered several new localities of Jurassic fossils, along the line of outcrop of the fossiliferous belt, previously noticed by different members of the Survey, as occurring on the Mariposa Estate. Several new species were found, which still remain to be worked up. During a portion of the year 1865 Mr. Gabb was employed in revising the palæontological materials of the Survey; but, owing to the limited appropriation by the Legislature of 1863-64, the field-work was necessarily on a very small scale.

A larger amount of money ( $\$ 15,000$ a year, for two years) having been granted by the Legislature of 1865-66, the work was taken up again nore vigorously at the beginning of 1866 . Mr. Gabb, assisted by Mr. F. E. Brown, commenced early in PAL. VOL. II.-B 
the year an exploration of the southern Coast Ranges, partly with the view of completing the palæontological collections in the Tertiary groups, and partly with the intention of working up the geology of the region, in which, at that time, a large anount of money was being expended for the purpose of ascertaining the value of the bituminous materials occurring there. This party continued in the field from January to June, adding largely to our knowledge of the geology of Los Angeles, Santa Barbara, and San Luis Obispo counties, and to our collection of Tertiary fossils.

Mr. Gabb continued his work northward from the Bay of San Francisco, accompanied by Mr. F. Coffee, during the months from July to November, and explored a large portion of Sonoma, Mendocino, and Iumboldt counties, a region of sedimentary rocks, chiefly of Cretaceous age, but not rich in fossils.

A party was at work during this summer in the High Sierra, in Mariposa and Tuolumne counties, under the charge of Mr. King; but their operations were exclusively confined to a region of granitic and volcanic rocks, and no additions were made by them to our palæontological materials.

Another party, under my own charge, in Plumas County, during the same summer, made a pretty careful exploration of the fossiliferous localities around Genesee Valley, and collected some new species. They were not successful, however, in finding, in Plumas County, any new localities of importance, although the region was pretty carefully examined. A party, under Mr. D'Heureuse, was also in the field during the summer of 1866, in Kern County, but not in a fossiliferous region.

In 1867 there was no work done in the fossiliferous forma- 
tions of California. The field-work was continued in Kern, Tulare, and Inyo Counties, by Mr. D'Heureuse and party; by Mr. Hoffmann and party in Mariposa and Tuolumne, and by Mr. Wackenreuder in the High Sierra, between Alpine and Plumas. Mr. Gabb, accompanied by Messrs. Wilson, Lölir, and Poston, explored the White Mountain Range, on the borders of California and Nevada. They continued their work east into the latter State (not at the expense of California), carrying their topographical and geological reconnoissance as far as the 116th meridian, including a large portion of the area between the 37 th and 39th parallels. They continued in the field until the end of October, as long as the season would permit. Their collections embraced a considerable number of fossils of various geological ages, from Lowier Silurian to Triassic.

A part of these was unfortunately lost in transitu; of the remainder, a portion has been worked up by $\mathrm{Mr}$. Gabb, and the results will be published in the Conchological Journal. All the Palæozoic fossils collected by the Survey, together with a considerable amount of palæontological material from rocks of this age, collected by different members of the corps, and a considerable number of interesting specimens contributed by others, have been placed in Mr. Meek's hands for examination, with a view to future publication of the results by the Survey, or otherwise. We are especially indebted to Mr. J. E. Clayton for an interesting collection from Silver Peak and vicinity, and to Mr. S. S. Lyon for one from the neighborhood of Pahranagat. Mr. Gorham Blake and Mr. A. Blatchly have also contributed valuable Palæozoic fossils (as well as Triassic and Jurassic), from various localities in Nevada.

The occurrence of rocks of Upper and Lower Silurian 
age in Nevada was first made known by the Geologieal Survey, in the Proceedings of the California Aeademy (see Vol. III, p. 307) in 1866. Previous to that, in May of the same year, a synopsis of what was known in regard to the age of the stratified roeks of Nevada, was communieated to the same Acadenin (see Proeedings, Vol. III, p. 266). The colleetions reeeived from Nevada at various times, together with the results obtained by myself in three visits to the State, and the important work of Mr. Gabb and party in 1867, have given us a pretty good idea of the geologieal strueture of Western and Southwestern Nevada. The "Survey of the 40th Parallel," organized by the U. S. Engineer Offiee in 1868, and plaeed in charge of Mr. C. King, formerly of the California Survey, will give us a large amount of detailed and reliable information in regard to the northern and eastern portions of the State, and it only remains to work up the southeastern eorner, a very interesting region, but one diffieult of exploration. To this part of the State I had hoped to be able to send a party during the summer of 1868 ; but cireumstanees have rendered it impossible for me to earry out my plans.

At the meeting of the National Aeadeny of Sciences, held at Northampton, in August last, Mr. Gabb read a paper giving the results obtained by himself in working over the eollections of the Geological Survey, in referenee to the subdivisions aud palæontologieal relations of the Cretaeeous and Tertiary roeks of California. In this paper Mr. Gabb proposes a new division of the Cretaceous of the Paeific eoast, whieh is referred to in the body of the present volume, and the leading features of whieh may here be sileeinetly stated.

The Cretaceous formation is found eovering large areas on the West Coast, from Vaneouver and the adjacent islands of 
the San Juan Archipelago, on the north, tlırough Washington Territory and Oregon to Southern California, as well as isolated patches in Eastern Oregon and in Mexico. Except that in Mexico, which seems to be an extension of one of the best-known members of the Texan Group, it is divided into four groups, as follows:

1st. The Tejon Grounp, the most modern member, the Division B. of Palæontology, Vol. I, is peculiar to California. It is found most extensively developed in the vicinity of Fort Tejon and about Martinez. From the latter locality it forms an almost continuous belt in the Coast Ranges to Marsh's, fifteen miles east of Monte Diablo, where it sinks under the San Joaquin plain. It was also discovered, by the different members of the Survey, at various points on the eastern face of the same range, as far south as New Idria, and, in the summer of 1866, by Mr. Gabb, in Mendocino County, near Round Valley, the latter locality being the most nortliern point at which it is as yet known. It is the only coal-producing formation in California.

This group contains a large and highly characteristic series of fossils, the larger part peculiar to itself, while a considerable percentage is found extending below into the next group, and several species still further down into the Chico Group. $\mathrm{Mr}$. Gabb considers it as the probable equivalent of the Maestricht beds of Europe.

$2 d$. The Martinez Group is proposed provisionally, to include a series of beds, of small geographical extent, found at Martinez and on the northern flank of Monte Diablo. It may eventually prove to be worthy of ranking only as a subdivision of the Chico Group.

3d. The Chico Group is one of the most extensive and important nembers of the Pacific coast Cretaceous. Its exact 
relations with the formation in Europe have not yet been fully determined, though it is on the horizon of either the Upper or Lower Chalk, and may probably prove to be the equivalent of both. It is extensively represented in Shasta and Butte counties, and in the foot-hills of the Sierra Nevada as far south as Folsom, occurring also on the eastern face of the Coast Ranges bordering the Sacramento Valley, at Martinez, and again in Orestimba Cañon, in Stanislaus County. It includes all of the known Cretaceous of Oregon and of the extreme northern portion of California, and is the coalbearing formation of Vancouver's Island.

4th. The Shasta Group is a provisional name, proposed to include a series of beds of different ages, but which, from our imperfect knowledge of the subject, cannot yet be separated; it includes all below the Chico Group. It contains fossils, seemingly representing ages from the Gault to the Neocomien, inclusive, and is found principally in the mountains west and northwest of the Sacramento Valley. Two or three of its characteristic fossils have been found in the vicinity of Monte Diablo, and one of the same species has been sent from Washington Territory, east of Puget Sound. Few, or none, of its fossils are known to extend upwards into the Chico Group.

\section{J. D. WHITNEY.}

Cambridge, Mass., Februiry, 1869.

Note.-Section 1, Part 1, was issued in February, 1866; the remainder of this volume was laid before the Philadelphia Academy, in a printed form, in December, 1868. 


\title{
PALEONTOLOGY OF CALIFORNIA.
}

\author{
V 0 I. I I.
}

SECTION I.

TERTIARY INVERTEBRATE FOS SILS.

$$
\text { P A R T I. }
$$

DESCRIPTION OF NEW SPECIES. 



\section{TERTIARY INVERTEBRATE FOSSILS.}

\section{CANCER L.}

C. Brewerir, n. s.

Pl. 1, Fig. 1.

HAND shorter, more robust and broader proportionally than that of C. magister, Dana; upper margin flattened and bordered by two well-defined ridges, each bearing about four tubercles; outer surface marked by five nearly obsolete ridges minutely tuberculated; fingers shorter than in C. magister, the movable one more curved and both with the denticles nearly obsolete.

Figure, natural size.

Locality: Near Santa Barbara. Pliocenc. Collected by Professor Brewer.

A single well-preserved hand, and some fragments were found by Professor Brewer, all in one locality, on the Cayeguas Ranch. The hand resembles closely that of the common coast species, in its principal features; but its broader and more robust form, the absencc of the marked serration on the back, and the curved finger, sufficiently distinguish it.

TRIPTERA, Quoy and Gaimard.

T. cLavata, n. s.

Pl. 1, Fig. 2.

SHELL small, subangulated on the sides, compressed and slightly constricted in the upper part, nearly elliptical in section below, pointed at the tip. Surface smooth or marked by a few very faint transverse undulations. Length about .3 inch.

P $\triangle$ L. VOL. II. -2 
Locality and position: From the Miocenc, in a boulder near Griswold's, in the Coast Range, on the road to New Idria.

But a single specimen was found in a boulder, associated with Pecten caurinus, Area microdonta, \&c., and somc other species, mostly indeterminate. I could detect no trace of the terminal septum, but this may be due to the extremc thinness of the shell and a portion of the interior being filled with crystals.

\section{TROPHON, Montf.}

\section{T. PONDEROSUM, n. $\mathrm{s}$.}

\section{Pl. 1, Fig. 3.}

Shell moderate in size, robust, thick; spire sub-elevated; whorls five, angulated in the middle, sloping above; suture irregularly impressed. Surface marked by eight or nine blunt varices, prominent and acute on the angle of the whorl, and commonly becoming more or less obsolete above and below; in the upper whorls the varices are replaced by mere elevated ribs, which become smaller until in the first three volutions they barely exist as undulations. Aperture broad; canal open, short, slightly recurved; columellar lip heavily incrusted; outer lip entire, subacute. Umbilicus well marked, but closed.

Figurc, natural size.

Localities and positions: From the Pliocenc of Kirker's Pass; also from the Miocene at Walnut Creek, Contra Costa County; and Griswold's, Monterey County, on the road to New Idria.

This finc species resembles somewhat T. Belcherii, Hinds, now living on the Coast, but can be at once distinguished by its smaller size, lower spirc, broader and more robust form, and by the number and character of the varices. Some very much mutilated specimens from Griswold's, probably referable to the present species, have a row of promincnt tubercles on the body whorl about half an inch below the angle. 


\title{
NEPTUNEA, Bolten.
}

Chrysodomus, Swains.

\author{
N. RECURVA, n. s.
}

Pl. 1, Fig. 4.

Shel of moderate size, fusiform; spire elevated, about as long as the mouth; whorls seven or eight (?) rounded on the sides, suture impressed. Surface marked by numerous, regular, rounded revolving ribs, with acute interspaces; these ribs sometimes show a tendency to alternation in size; the upper whorls sometimes, though not in all specimens, show a few faint longitudinal ribs. Aperture broad in the middle, narrowed in advance; lips simple; canal long, narrow, and very strongly recurved.

Figure, natural size.

Localities: Abundant in the Miocenc at the Arroyo San Antonio, near Tomales Bay. Also found in the same formation at Foxin's Ranch, in Santa Barbara County, on the El Toro Ranch, and near Griswold's, in Monterey County; and in Morgan Valley, Lake County.

This strongly-marked species seems to be peculiar to the middle and upper portions of the Miocene formation.

It is allied to $N$. (Fusus) exilis, Con., of the Virginia Miocene (see Foss. Tert. Form. pl. 49, fig. 6); but the mouth is proportionally larger, the canal is more recurved, and it wants the "longitudinal rounded ribs" of that species. The rcvolving ribs in this species also are markedly rounded, while in the Eastern shcll they are described as acute.

METULA, H. and A. Adams.

? M. REMONDII, n. s.

Pl. 1, Fig. 5.

SHelr broadly fusiform, spire elevated; whorls four or five (?) subangulated; suture impressed. Surface marked by numerous small revolving riks, rounded with acute interspaces, and more 
or less regularly alternating in size; these ribs are crossed by strong lines of growth, so as to present in some specimens an approach to a regular reticulation. Body whorl three-fourths of the whole length of the shell, broadly and regularly convex in the middle, with usually a revolving depression just below the suture; sometimes this groove or depression is replaced by a flat space. Mouth long and narrow; inner lip slightly incrusted; outer lip unknown; canal straight.

Figure, natural size.

Locality and position: From the Mioeene of Arroyo San Antonio, near Tomales Bay.

The spccimens are all preserved in a very hard gray sandstonc, and I have been unable to expose the outer lip, so as to ascertain whether the characters exist on which the authors have mainly depended in instituting the genus. The form of the shell, howcver, and all the other characters are so nearly in accordanee with thcir typc, that I have ventured to place the species under their generie name provisionally.

\section{CLAVELLA, Swains.}

\section{Gravida, n. s.}

Pl. 1, Fig. 6.

SHELL short, robust; spire moderately elevated, whorls five, suture channelled; body whorl bordered above by a strongly marked rim, adjoining the suture, and which extends to the upper whorls, though less distinctly marked; below this band is a slight depression of variable depth, and from that the volution swells rapidly. Surface marked by two or three rows of nodes on the middle of the whorl, and by fine revolving lines. Aperture broad in the middle, narrowing in advance; canal moderate, slightly twisted; inner lip slightly incrusted.

Figure, natural size.

Localities: From the Mioeene; abundant south of Martinez, where it was collected by Dr. Fish and Mr. Mathewson. Also figured in the Paeific Railroad 
Report, Vol. 5, Pl. 7, Fig. 63, from Ocoyo or Pose Creek, Tulare County, where casts were found.

It is possible that the speeimens figured as No. 67 , on the same plate, under the name of Natica geniculata, Con., may belong to this spccies, though the drawing is too imperfect for a satisfaetory determination.

\section{C. sinuata, 1. s.}

P1. 1, Fig. 7.

Shell elongated, rather slender; spire low, convex; whorls four; suture deeply channelled, bordered by a thickened rim; body whorl convex in the middle, broadly grooved above, and excavated below. Surface marked by numerous fine revolving lines, and in the upper whorls by numerous radiating ribs. Aperture long and narrow; columellar lip sinuous, slightly incrusted; outer lip simple; canal slightly recurved.

Figure, natural size.

Locality: From the Miocene of Walnut Creck, Contra Costa County. Rare.

But two specimens of this curious shell have bcen found; one of which is of the size figured, the other not more than half as large.

\section{PLEUROTOMA, Lam.}

Turris, Bolt., not Humph.

\section{P. (Surcula) Carpenteriana.}

Pl. 1, Fig. 8.

(P. (S.) Carpenteriana, Gabb; Proc. Cal. Acad. Nat. Sci., 1865, p. 188.)

SHeLl large, fusiform; spire about as long as the mouth; whorls eight, subflattened on the sides, slightly concave near the suture, and very slightly convex below; suture impressed Surface marked by numerous fine revolving ribs, rounded and sometimes alternating in size, especially on the lower part of the body whorl. Aperture narrow; inner lip faintly incrusted; outer 
lip acute; sinus very broad and shallow; canal moderate, slightly twisted.

Figure, natural size.

Locality: From the Post-Pliocene, Santa Barbara.

This beautiful spccies is also found living on the coast of California, a beach specimen having been found at San Dicgo, and another dead specimen was dredged by Dr. Cooper, at a depth of 120 fathoms, in the samc vicinity. The colors, when living, are a brownish-orange, with broken revolving bands of a light reddish-brown; thesc bands usually occur on the larger ribs, and are most closely placed on the middic of the shell.

In my description, above quoted, I inadvertently mentioned two fossil specimens. Only one has yet been found, but that is the most perfect specimen yet known of the species, exccpt in color.

\section{P. (S.) Tryoniana, n. s.}

Pl. 1, Fig. 9, and $9 a$.

Sinell large, fusiform; spire about as long as the mouth; whorls about seven or eight, angulated in the middle, sloping concarely above, and very slightly convex below. Surface ornamented by a row of nodes on the angle of the whorl, and by numerous fine revolving ribs. Aperture narrow; inner lip slightly sinuous and incrusted; posterior sinus of outer lip broad and shallow; canal moderate, nearly straight.

Figures, natural size.

Locality: From the Post-Pliocene of San Pedro

This shell is closely allied to the preceding; but can be at once separated from it by the angulation of the whorls and the presencc of the tubcrcles. The outline drawing, fig. $9 \alpha$, is restored from the lincs of growth for the purpose of showing the form of the lip. The spccics has not, as yct, been found living.

\section{P. (S.) PERVERSA.}

Pl. 1, Fig. 10.

(P. (S.) perversa, Gabb; Proc. Cal. Acrd. Nat. Sci., 1865, p. 183.)

Shell small, sinistral, elongate, slender; spire high, apex usually bent to one side; whorls eleven to twelve, convex, 
two nuclear whorls rather loosely twisted and more convex than the others; suture deep; body whorl nearly half of the entire length of the shell. Aperture broadest in the middle, narrowing regularly in advance; inner lip moderately incrusted; outer lip simple; siuus rather broad, shallow, rounded, and adjoining the suture; canal of variable length, often much larger than in the specimen figured. Color, in living specimens, a light reddishbrown.

Figure, natural size.

Locality: San Pedro, from the Post-Pliocene; not rare. Also found living, by Dr. Cooper, at a depth of sixty fathoms, off Santa Catalina Island.

\section{P. VoYI, n. s.}

Pl. 1, Fig. 11.

Shell somewhat resembling the preceding species, but dextral and much broadcr; spire elevated; whorls perhaps seven or eight (apex broken), very convex; suture decp. Surface smooth, or only marked by lincs of growth. Aperture broad; inner lip incrusted; outcr lip acute; posterior sinus deep, romded at the base, placed a little distance from the suture; canal slightly bent.

Figure, very slightly magnified; about onc-fourth inch longer than the specimen.

Locality: Found by Mr. Voy near Humboldt Bay, below Bear River, Humboldt County: Miocene or Pliocene (?).

\section{CLATHURELLA, Carpenter.}

Defrancia, Millet, non Müll.

\section{Conradiana, n. s.}

Pl. 1, Fig. 12.

SHeLl small, robust, broadly fusiform; spire hign; whorls seven and a half, the first smooth and round, the remainder an- 
gulated; concave above, convex on the side; suture very indistinct. Surface marked by ten or twelve large, rounded, radiating ribs, crossed by square elevated ribs, with sometimes smaller ones interposed; on the upper or concave portion of the whorl are six or seven fine linear revolving ribs; of the large revolving ribs, three or four only appear on the upper whorls, the body volution showing about fourteen. Aperture narrow; inner lip thinly incrusted; outer lip acute on the edge, thickened behind; posterior sinus narrow, deep, oblique, bordered by a thickened rim and almost adjoining the suture.

Figure, nearly four times the length of the speeimen.

Locality: Not eommon, in the Post-Plioeene of Santa Barbara.

Speeimens resembling this, but differing a little in the minute details of seulF ture, were found by Dr. Cooper, in dredging off the shores of Santa Catalina Island. They should probably be plaeed under the same speeifie name.

\section{RANELLA, Lam.}

\section{R. Mathewsonit, n. s.}

Pl. 2, Fig. 13.

Shell moderate in size, robust, sub-compressed; spire high; number of whorls unknown; suture strongly impressed, bordered by a small, though abrupt truncation of the succeeding whorl; varices prominent, rounded, continuous. Mouth small; inner lip heavily incrusted; canal abruptly recurved. Surface marked by numerous, rounded, longitudinal ribs, crossed by square revolving ribs, with smaller ones interposed, and with flat interspaces.

\section{Figure, natural size.}

Locality and position: From the Mioeene, south of Martinez; a single speeimen found by Mr. Mathewson.

This shell can be at onee distinguished by its eompaet form and the alternating revolving ribs. No allied speeies, either fossil or reent, has been found in California. 


\section{CUMA, Humph.}

C. Biplicata, n. s.

Pl. 2, Fig. 14.

SHewL small, thick, angular; spire elcvated; sides ncarly straight, about as long as the mouth, in very old specimens a little longer; whorls five and a half to six, the first two smooth and round, the others concave and sloping above; suture faint, covered by a thin layer from the succeeding whorl; body whor prominent and subangulated in the middle, bordered by a marked. angular rib at the top, which passes up on the preceding whorls; ornamented on the sides by about twelve short longitudinal nodose ribs, and by about eight or nine smaller revolving ribs, the uppermost of which crosses the top of the longitudinal ribs; these revolving ribs become fainter in front, until on the anterior fourth of the shell they are nearly obsolete. Aperture broad, angular above; columellar lip heavily incrusted; outer lip simple; canal slightly bent. Umbilicus broad, imperforate, bordered by two very strong oblique revolving ridges; anterior sinus deep, narrow, and oblique.

Figure, natural size.

Locality and position: Not rare in the Miocene, south of Martinez, where it has becn collected by Mr. Mathewson and Mr. Voy.

\section{ANCILLARIA, Lam.}

\section{A. Fishir, 11. s.}

Pl. 2, Fig. 15.

SHeLu small, broad; spire high; whorls six; suture covered. Aperture narrow, acute posteriorly, broad in advance; inner lip moderately incrusted, with a single small fold in advance; outer lip acute, anterior sinus decp.

PAL. VOL. II. -3 
Figure, natural size.

Locality and position: From the Miocene, south of Martinez. Collected by Dr. Fish, of Martiñez, and Mr. Mathewson.

This shell has few tangible charactcrs except the high, acute spire, and the general outline. The small mouth will at once distinguish it from Olivella biplicata, and its size, from O. Pedroana (batica of Carpenter).

\title{
COLUMBELLA, Lam.
}

\author{
S. G. Alia, H. and A. Ad. \\ C. (A.) Richthofeni, n. $s$.
}

Pl. 2, Fig. 16.

Shell clongated, slender, subfusiform; spire high; whorls five and a half to six, slightly convex on the sides; suture deep. Surface smooth, or marked only by irregular, stray lines of growth, except on the anterior part, where there are a few oblique revolving lines. Aperture subquadrate; inner lip incrusted and bearing a few linear folds or teeth anteriorly; outer lip thickcned and crenulate within; canal narrow and slightly curved.

Figure, magnified; length of largest specimen about .5 inch.

Localities and position: From the Pliocene, on the Russian River, collected by Baron F. Von Richthofen, and from the same formation in San Francisco County, in the bluff's on the coast, by M. Rémond.

NEVERITA, Risso.

N. CALLOSA, n. s.

Pl. 2, Fig. 17, $17 a, 17 b$.

SheLL obliquely subglobosc, compact; spire very small; whorls four and a half, almost completely hidden; suture sharp; body whorl broadly convex. Aperture narrow, very oblique, acute behind, round in advance; outer lip simple; inner lip covered by an unusually heavy callus which covers the umbilicus, is cmar- 
giuate above the umbilical region, and nea: the lower edge is marked by an oblique groove.

Figures, 17 and $17 a$, natural size; $17 b$, magnified view from above.

Locality: Walnut Creek, from the Mioeene. $\boldsymbol{\Lambda}$ form somewhat resembling this has been found by Dr. Cooper at San Pedro; but it differs in the spire being more developed, and in the body whorl being less oblique and more expanded laterally.

\section{CANCELLARIA, Lam.}

S. Gen. Euclia, H. and A. Ad.

C. (E.) Tritonidea, n. s.

Pl. 2, Fig. 18.

Shell large, robust; spire elevated, nearly as long as the mouth; whorls five, the first two and a half round and smooth, others bluntly angulated and descending straight, or with a slightly convex slope above; suture irregular, nearly obliterated by small lamellæ passing up from the succeeding whorls, in continuation of the lines of growth. Surface marked by a series of blunt nodes on the angles of the whorls, from which ribs or ridges, very irregularly dispersed, run to the suture above and downwards almost to the base of the shell; these are crossed by a large number of irregular revolving ribs of small size. Aperture suboval, broadest towards the base; inner lip heavily incrusted, bearing two large and one or two smaller folds. Umbilicus covered. Canal almost obsolete, slightly bent backwards.

Figure, natural size.

Locality: Pieked up on the beach near San Pedro, by Dr. Ccoper, and supposed to have washed out of the Post-Plioeene beds of that locality. This opinion is strengthened by the appearanee of the speeimen, it resembling in cvery respeet a fossil, rather than a mere beaeh specimen of a living shell; and, in addition to that, no sueh shell, or even a fragment, has ever been found to indicate that the speeies may still be living. 
C. (E.) vetusta, n. s.

Pl. 2, Fig. 19.

SHELi small, spire not so long as the mouth; whorls five or five and a half, increasing rapidly in size, angulated and truncated above, sloping convexly inwards below; suture moderately well marked, undulated. Surface marked by longitudinal ribs commencing at the suture, passing obliquely backwards to the angle, and then downwards to the anterior end; these are crossed by finc revolving square ribs, with broad, flat interspaces, and with sometimes smaller oncs intercalated. Aperture long, biangular above, and narrowing in advance; inner lip heavily incrusted, bearing two heavy folds, and two or three fine ones above. Umbilicus narrow, covered by a very thick expansion of the inner lip, and in one specimen showing a slight perforation.

Figure, magnified. Length about one inch.

Locality: Found thus far only in the Miocene, south of Martiñez. Two or three specimens collected by Mr. Mathewson.

It is possible that a further study of the species would prove that the perforated umbilieus mentioned above is not a constant eharacter. It scems to be, in this instance, rather the result of accident than a specific charaeter.

\section{BITTIUM, Leach.}

B. ASPERUM.

Pl. 2, Fig. 20.

(Turbonilla aspera, Gabb; Proc. Philada. Acad. Nat. Sci., 1861, p. 368.)

Shenc long, slender; whorls twclve or thirteen; nuclear whorls smooth, others marked by ten or twelve strong, longitudinal ribs, crossed by four or five sharp, filiform, revolving ribs with broad interspaces; suture deep. Under surface smooth, or marked by one or two ribs near the angle. Mouth longer than broad, produced below and slightly expanded at the columellar angle; lips acute. 
Figure, magnified. Length about .35 inch.

Locality: From a Post-Pliocene Marl, Santa Barbara Collected by Dr. A. L. Hecrmann and others.

\section{MELANIA, Lam.}

\section{TAYLORI, n. s.}

Pl. 2, Fig. 21.

Sheld slcuder, elongate, many-whorled; whorls about twelve, flattened on the sides; suture impressed. Surface marked by three or four sharp revolving ribs, crossed by numerous curved longitudinal ribs, forming a prominent flattened tubercle at each intersection; there are about twenty-five of these longitudinal ribs to each volution; under side of the body whorl ornamented by four or five sharp revolving ribs. Aperture suboval; produced in advance; inner lip incrusted; outer lip simple.

Figure, natural size.

Locality: From a fresh-water Tertiary deposit on Snake River, Idaho Territory, on the road from Fort Boisé to the Owyhee mining country. Collected by Mr. A. Taylor.

\section{LITHASIA, Lea.}

L. ANTIQUA, n. s.

PI. 2, Fig. 22.

Suelu subglobose; spire moderately elevated; whorls five, rounded; suture strongly impressed. Surface smooth. Aperture oblique, suboval, subacute above, rounded and slightly expanded below; outer lip simple; inner lip heavily incrusted. Umbilical region flattened, bordered by an angular carina, and covered by an expansion of the inner lip.

Figure, slightly magnified.

Locality: With the preceding. Associated with these two shclls is a little bivalve, perhaps a species of Sphorium, not in a sufficiently good state of preservation for description. 


\section{LITTORINA, Fer.}

\section{Rémondi, n. s.}

P1. 2, Fig. 23, $23 a$.

Sireld small, eonieal, thiek; spire elevated; whorls five, nearly flat on the sides, subangulated at the base; suture impressed, under side obliquely flattened. Aperture subtriangular; outer lip simple; inner lip somewhat flattened, thiekened, bordered by a more or less marked rim, behind whieh is generally a faint umbilical depression. Surface smooth, or marked by a few faint spiral lines. The lower angle of the whorls is variable; sometimes it is roundly subangulated, and at others it is bordered by a prominent thickened rib or band.

Figures, magnified views of the two extreme forms. Length about half an ineh. Locality and position: From the Pliocene of Kirker's Pass. Found only in a single bed, and eollected by Mr. Rémond.

\section{TURRITELLA, Lam.}

\section{T. Hoffmanni, n. $\mathrm{s}$.}

Pl. 2, Fig. 24.

SHeLl large, many-whorled, elevated; suture deep; whorls inereasing very gradually in size, flattened on the sides, slightly bevelled above and below the suture, and a little depressed in the middle; under surfaee nearly flat, or a very little eonvex. Surface marked sometimes by a few faint revolving lines. Aperture subquadrate.

Figure, natural size.

Locality: From a white Miocene limestone in the extreme southern eorner of the Maxima Martiñez Raneho, Santa Clara County.

This is the largest Turritella yet found in the State, and bears a elose resemblance to $T$. Saffordii, nub., of the Cretaceous. It differs mainily in the suture not being so elosely impressed as in that species. 


\section{TROCHITA, Schum.}

T. FILOSA, n. s.

Pl. 2, Fig. 25, 25 a.

SHELL irregularly conical, variable in height and outline, some times half as high as broad, sometimes height and width equal, in outline sometimes circular, in other specimens very oblique; volutions about two and a half; suture obsolete; internal plate concave; outer edge regularly rounded. Surface marked by numerous fine radiating lines, often dichotomous.

Figures, natural size, from a very regular average spccimen.

Locality: From the Miocene of Walnut Creek, Contra Costa County.

A single specimen was found with these, mucl highcr than the average spccimens, with a strongly marked spiral depression, and without the radiating lines It will probably prove to be a distinct species, but in the absence of more mate rial I do not feel warranted in naming it.

\section{PACHYPOMA, Gray.}

\section{?P. BIANGulata, n. s.}

Pl. 3, Fig. 26.

SHeLt large, conical; spire high; whorls five or more, sloping above, flattened on the sides; suture small. Surface oruamented by revolving rows of small tubercles; angles of the whorls marked by a larger series than on the rest of the shell; under surface nearly flat, marked by four or five revolving ribs with marked flat interspaces. Aperture large, subquadrate; outer lip simple; inner lip and umbilical region unknown.

Figure, natural size.

Locality and position: A single, somewhat injured speeimcn, from the Miocene, south of Martinez; collected by Mr. Mathewson.

Owing to the crystalline charactcr of the shcll, and the toughness of the matrix, I have not been able to expose all of the important parts of the surface of this 
shell; the generic relations must therefore be left in doubt until further specimens are found exhibiting more of the details of the under surface. In some of its details, the present species resembles $P$. gibberosum living on the coast, but its strongly biangular form will serve to distinguish it.

\section{TURCICA, H. and A. Ad.}

Subgen. PTYCIIOSTYLis, Gabb.

(Id., Gabb; Proc. Cal. Acad. Nat. Sci., Jan. 1865, p. 187.)

Animal unknown. Shell resembling Thalotia and Calliostoma; conieal, not umbilicated; spire elevated; aperture subquadrate, internally pearly; columella truneated, bearing an oblique fold on the anterior end and one above; outer lip and base aente, slightly thickened behind; umbilieal region slightly excavated, and covered by a thin expansion of the inner lip.

T. (P.) COFFEA.

Pl. 3, Fig. 27.

(P. coffea, Gabb ; Proc. Cal. Acad. Nat. Sci., Jan. 1865, p. 187.)

Shell eonieal; spire elevated; sides flattened; whorls seren, first one smooth and round, suceeeding ones subangulated at the lower margin, obliquely flattened above; body whorl convexly subtruncated below; suture ehannelled. Surfaee marked by a row of comparatively large tubercles on the upper margin of the whorl, adjoining the suture, and by a variable number of revolving, finely tubereular ribs on the sides; there are usually from six to ten on the side, and about the same number on the under surfaee of the body whorl; these tubercles are very small, and are generally arranged in an irregular quincunx; the under surlaee of the body whorl, just below the angle, is marked by a deep revolving groove. Aperture subquadrate; outer lip and base acute; inner lip narrowly emarginate beyond the truneated end of the eolumella. 
Figure, slightly magnified.

Locality: From the Post-Pliocene of San Pcdro and Santa Barbara. Also found living at Monterey. The epidermis in the living specimen is of a rich coffecbrown. The living specimen, from which the original description was written, was immature, having a full whorl less than the mature fossils now under consideration; the shell was also much thinner.

CALLIOSTOMA, Swains.

\title{
Zizyphinus, Gray.
}

C. TRICOLOR.

\author{
Pl. 3, Fig. 28.
}

(C. tricolor, Gabb ; Proc. Cal. Acad. Nat. Sci., Jan. 1865, p. 186.)

SHeLl small, conical; spire elevated, whorls seven; first whorl smooth, others concavely to convexly sloping above, more or less distinctly biangular on the margin; suture small, impressed. Surface closely and minutely marked with fine revolving granular ribs. Aperture subquadrate, internally nacreous; inner lip thick; outer lip and base acute.

Figure, a magnified view of a fresh specimen. Length about .5 inch.

Locality: Post-Pliocene, San Pedro.

Dr. Cooper has collected this shell, living, along the coast from San Diego to Half Moon Bay. Its peculiar colors suggested the specific name. It is a lightbrown, banded by spiral lines of purple interrupted by white spots.

Figures 29 and 30 represent two of the many forms which havc been discovcred in the California Miocene, of which sufficient material has not yet been accumulated for satisfactory determination. Both of these specimens were found in the San Emidio Cañon, twenty miles west of Fort Téjon, Los Angelos County, in a hard Miocene sandstone.

PAL. YOL. II. -4 


\title{
ZIRPHÆA, Leach.
}

\author{
Z. DENTATA, n. S. \\ Pl. 3, Fig. 31, $31 a$.
}

SheLt large, subcylindrical, thin; ends broadly gaping; beaks anterior to the middle; covered by the dorsal plate; posterior dorsal margin of valves thin and sharply reflexed. Surface of anterior third of shell marked by serrated, squamose plates, as in Z. crispata; a faint line or rib passes between the middle and posterior third of the width, from the beaks obliquely down to the base; dorsal plate heavy, compressed, and divided into two concave surfaces by a sharp, angular ridge, commencing at the posterior end, and running forwards, slightly curved, ending in a tooth at a point about a third of the length of the plate from the anterior extremity.

Figures, natural size; fig. $31 a$, represents the dorsal plate.

Locality: A single speeimen from the Plioeene beds at the east end of Kirker's Pass, Contra Costa County.

This shell is closely allied to $Z$. crispata; but differs in its more reguiarly eylindrieal form, the marked reflection of the posterior dorsal margin, and the angular dentated dorsal plate.

\section{PANDORA, Brug.}

P. SCAPHA, n. s.

Pl. 4, Fig. 32.

SheLL small, irregularly subquadrate; right valve flat; left valve slightly convex; beaks small, about a third of the width from the anterior end, which is prominent and regularly convex; cardinal margin slightly concave near the beaks, straight behind; base broadly and regularly curved; posterior end narrow and prominent. Surface marked by a few lines of growth.

Figure, magnified about a third. 
Locality: From the Mioeene, west of Martiñez; colleetion of Mr. Rémond. The species appears to be rare. I have never seen it exeept in two small slabs, in whieh, however, a large number of speeimens are closely packed.

\section{HEMIMACTRA, Swains.}

$$
\text { Spisula, Gray. }
$$

H. LENTICULARIS, n. $\mathrm{s}$.

Pl. 4, Fig. 33.

Shell large, flattened, thin, inequilateral, irregularly subquadrate; beaks eccentric, large, slightly curved forwards and inwards; anterior end broadly concave below the beaks, and convex below; base and posterior end regularly convex; cardinal margin sloping, nearly straight. Hinge teeth slender. Pallial sinus moderately deep, round at the base. Surface marked by irregular lines of growth.

Figure, natural size of an average sized speeimen, with a transverse thiekness of about 1.3 ineh. One speeimen is 3.8 inehes long and 4.2 wide.

Localities and position: From the Mioeene, south of Martiñez, collected by Dr. Fish; also, west of Martinez, on the south shore of the Straits of Carquinez, and at Griswold's, near New Idria, Monterey County.

\section{MULINIA, Gray.}

\section{? M. Densata.}

Pl. 5, Fig. 35 .

(M. densata, Conrad, pars; P. R. R. Report, vol. vi, p. 71, pl. 3. fig. 12.)

SHell thin, equivalve, inequilateral; beaks large, nearly central; anterior cardinal margin broadly concave, posteriorly sloping with a slight convexity; base broadly and regularly convex; anterior and posterior ends slightly truncated. Hinge small; teeth slender.

Figure, natural size, from a speeimen rather smaller than the average. 
Localities: Common in the Miocene, south of San Pablo Bay; south of Martiñez; near Walnut Creek, and in the hills cast of Oakland, Contra Costa County; San Emidio Ranch; west of Fort Tejon (and Santa Barbara, Con.).

From its extreme thinness, this shell is almost always found very much distorted. The specimen figured by Mr. Conrad had been pressed out of its natural shape, and probably, having bcen misled by this inconstant form, he has confounded two species, widely different in character, under the same name.

\title{
SCHIZODESMA, Gray.
}

\author{
S. ABSCISSA, n. s.
}

Pl. 4, Fig. 34, $34 a$.

Shell large, thick, irregularly subquadrate; beaks large, prominent, pointed forwards, nearly central; anterior end obliquely truncated; base broadly rounded; posterior end very strongly and abruptly truncated, the truneation ending at an angular ridge which passes from the beaks to the posterior basal angle. Surface marked by rough, irregular lines of growth. Hinge composed of large, robust tecth; lateral teeth long and thick.

Figures, natural size. Fig. $34 \alpha$, represents the postcrior end.

Localitics: South of Martiñez; ncar San Pablo Bay and Walnut Creek, Contra Costa County; Miocenc.

From the character of the hinge, and the fact that this and the preceding species are often found associated, it is probable that this is the species which Mr. Conrad confounded with his Mulinia densata; although the shape of the shell precludes the possibility that this is the form to which he intended to apply the name.

\section{PSEUDOCARDIUM, Gabb. N. Gen.}

Sneld thick, heavy, cquivalve, resembling Lawicardium externally; ligament internal. Lunale cordate, deeply impressed. Hinge composed of a large cartilage pit and in the left valve, a $\mathrm{V}$-shaped tooth articulating in a corresponding depression on the opposite valve; two lateral teeth in each valve, very large and prominent; these teeth are comparatively narrow in the young 
shelis, but in adult specimens attain an unusually large size; pallial line unknown.

From all the charaeters of the hinge, and the internal position of the ligament, this shell is evidently one of the Mactride. Its external form, which induced Mr. Rémond to plaee it in the genus Cardium, has also suggested the generic name.

\section{P. GabBit.}

Pl. 6, Fig. 45, $a, b, c$, and $d$.

(Cardium Gabbii, Rémond; Proc. Cal. Academy, 1863, vol. 3, p. 13.)

ShELL large, thick, ventricose, elongated cordate; beaks very large, strongly incurved and lightly pointed forwards; anterior end sloping, rounded below, slightly concave above; posterior more or less distinctly truncated; base regularly convex. Lunule broadly cordate, very deeply impressed. Surface irregularly roughened by strong lines of growth.

Figures, natural size.

Localities: From the Pliocene, near Kirker's Pass, and rare in the Miocene, south of Martiñez; also in the Sierra Bonita, Monterey County.

\section{GARI, Schum.}

Subgen. PSAMMOCOLA, Blainv.

$$
\text { G. (P.) ALATA, n. s. }
$$

Pl. 5, Fig. 36.

Shell oval, thin, compressed, equivalve; anterior end a little the narrowest; beaks small, central; anterior and posterior ends regularly rounded; base broadly convex; anterior cardinal margin expanded into a long narrow car, marked by one or two radiating grooves. Hinge composed of two prominent oblique teeth in cach valve. Surface marked by a few irregular lines of growth.

Figure, natural size.

Locality: From the Pliocene beds near the cast ond of Kirker's Pass 


\section{VENUS, L. \\ V. Kennerlyi, Rve.?}

Pl. 5, Fig. 37.

SHELL thin, subcompressed, rounded-subtriangular; beaks small, prominent, anterior; anterior and posterior ends about equal; base broadly rounded; posterior eardinal margin sloping convexly. Lunule small, elongate eordiform, flat. Surface marked by numerous irregular coneentrie undulations and fine striæ; internal margin entire, not crenulated. Pallial sinus deep, oblique, and narrow at the base.

Figure, natural size.

Locality and position: From the Miocene, at Griswold's, on the road to New Idria, Monterey County.

I have not had an opportunity of examining Rceve's figure or description of his species, and from the very meagre note on the species in Carpenter's last Brit. Ass'n Catalogue, I am unable to determine whether this should be referred to $\boldsymbol{V}$. Fennerlyi, or not. Should it prove distinct, I propose the name of V.pertenuis.

\section{MERCENARIA, Schum.}

\section{M. perlaminosa, Con.}

Pl. 5, Fig. 38.

(M. perlaminosa, Con.; Proc. Phila. Acad. Nat. Sci., 1855, p. 267.)

SiIELL, large, thiek, rounded subquadrate, very inequilateral; beaks anterior; anterior end exeavated under the beaks, rounded below ; hase broadly rounded; posterior end eonvexly truneated; cardinal margin slightly eonvex. Surface closely marked by numerous prominent, reeurved, lamelliform coneentrie ribs. Lunule marked by an impressed line. Hinge robust. Pallial line strong, siuus small, oblique, aeute at the base; museular sears nearly 
equal in size; margin minutely crenulated; the purple color between the pallial line and the base is very persistent.

Figure, natural size.

Locality: From the Post-Pliocene beds on the beaeh at Santa Barbara.

Allied to V. (Mercenaria) Ducatelli, Conrad, of the New Jersey Miocene, but much broader posteriorly, and the ribs are more numerous than are represented in the figure of that speeies.

\section{CHIONE, Megerle.}

\section{Mathewsonit, n. s.}

Pl. 5, Fig. 39.

SHELL very thick, obliquely cordate, very inequilateral; beaks anterior; anterior end sinuous; base broadly rounded; posterior end prominent, narrow. Lunule lanceolate, impressed. Surface marked by numerous irregular, concentric ribs; adjoining the cardinal edge and posterior to the beaks, is a narrow flat space running almost to the posterior end of the shell; inner margin entire, not crenulated. Pallial sinus deep; lower side curved, upper side straight.

Figure, natural size.

Locality and position: Not rare in the Mioeene, south of Martiñez; collected by Mr. Mathewson and Dr. Fish.

This shell is quite variable in outline, though all of the other characters are very constant. It seems relatcd to $C$. succincta $=$ Nuttallii and Californica, but differs in the entire absenee of radiating ribs.

\section{Whitneyi, n. s.}

Pl. 5, Fig. 40.

Suelt thin, oblique, rounded subcordate; beaks small, produced, pointing forwards; anterior end rounded, prominent, excavated above; base regularly convex; posterior cardinal margin rapidly descending, convex. Surface marked by numerous fine 
concentric ribs, abrupt on the upper side, sloping towards the base.

Figure, natural size.

Locality and position: A single specimen collected by Prof. Whitney, near Martiñez. Appears to be from the Miocene formation, from the eharacter of the matrix.

Related to C. foveolata, Sby.; but differs in not being so produced behind, and in being more regularly striate.

\title{
CALLISTA, Poli.
}

\author{
C. Voyi, n. s.
}

Pl. 5, Fig. 41.

SHeLl broad, inequilateral; beaks in advance of the middle: cardinal margin nearly straight and sloping; anterior end very much produced, rounded and narrow; posterior end obliquely truncated; base broadly and regularly rounded. Surface marked by numerous irregular and rather strong lines of growth.

Figure, natural size.

Locality and position: From the Mioeene or Plioeene near Humboldt Bay, below Bear River, Humboldt County; eolleeted by Mr. Voy.

This fine shell belongs to the type of C.gigantea, and is the only species of this group yet found in California. From gigantea it is easily distinguished by its broader posterior end, by the anterior end being shorter, more sloping above and narrower. The eharacter of the surface is also different.

\section{DOSINIA, Scopoli.}

D. Staleyr, n. $\mathrm{s}$.

$$
\text { Pl. 7, Fig. } 42 .
$$

SreLl subcircular, thick, very inequilateral; beaks large, prominent, anterior. Lunule faint, rather large, slightly sunken. Surface smooth, marked by a few lines of growth near the base. Hinge robust. 
Figure, natural size.

Locality and position: From the Pliocene on Mark West Creek, a braneh of Russian River, Sonoma County; discovered by Mr. V. S. Staley, after whom it is named.

This shell ean be at once distinguished from $D$. ponderosa, whieh is quite enmmon in the Tertiary of California, by its less eireular outlinc, the beaks being plaeed more in advance, by its being more eonvex, by its less strongly marked lunule, a differently shaped hinge, and its smooth surfaee.

\section{Conradi, n. s.}

Pl. 5, Fig. 43.

(D. alta, Con.; Proe. Philada. Aeademy Nat. Sei., 1856, p. 315; not D. alla, Dkr.; nor D. alta, Con.; P. R. R. Rep., V. 5, p. 320, pl. 2, f. 2: D. alta, Con.; P. R. R. Rep., Vol. 6, p. 71, pl. 3, fig. 13, 13 a.)

SHELl rather small, thin, very elongated; beaks prominent, margins rounded. Lunule deep. Surface marked by concentric lines of growth.

Figure, natural size.

Localities: San Enidio Raneh, near Fort Tejon; also "Monterey" (Dr. Newberry). From the Mioeene.

The original of Mr. Conrad's figure having been a distorted speeimen, I have prepared the present drawing for the purpose of showing the true outline of the speeies. Unfortunately his name was preoeeupied, and I have therefore dedieated the species to the pioneer of Tertiary Palæontology, not only for California but of the Atlantic States as well.

TAPES, Megerle.

? T. TRUnCATA, n. s.

- Pl. 7, Fig. 44.

SHewl large, thin, transverse; beaks in advance of the middle, pointed forwards; anterior and posterior ends nearly equal, rounded; base broadly and regularly convex. Surface marked by numerous small, radiating ribs, nearly obsolete posteriorly. PAL. VOL. II.- 5 
and erossed by fine concentrie, subimbrieated lines; a sharp, angular ridge runs from the beaks baekwards, nearly parallel with the cardinal edge, between whieh and the ridge is a flat spaee sloping inwards; internal margin finely erenulated.

Figure, natural size.

Locality and position: From the Miocene, at Griswold's, Monterey County.

But a single specimen was found, and $I$ have becn unable to expose the hinge. $I$ have thercfore not been able to determinc positively the genus.

\section{CYRENA, Lam.}

\section{Californica, n. s.}

Pl. 7, Fig. 45.

Shell very variable in outline, suboval or subquadrate, more or less oblique; beaks prominent, varying in position from almost median to nearly submarginal, the variation being prineipally due to the greater or less prominenee of the anterior end, and the greater or less obliquity of the shell; anterior end usually produced and most prominent above; posterior end more or less distinetly truneated, the truneation being oblique. Surfaee marked by numerous, irregularly disposed eoneentric lines. Ilinge teeth sharply prominent; hinge plate slightly expanded on the ligament margin, extending a little beyond the general outline of the shell. Pallial margin strongly impressed.

Figure, natural size.

Locality and position: Very abundant in the Pliocene, near the east end of Kirker's Pass.

This species is the most variable in outlinc of any fossil $I$ have seen in California, except the oysters and similar shells. No two specimens have the same shape, and by selerting a suitable series, differences cnough could be found to make three or four species, sufficiently distinct, on paper. The large number of specimens, however, at my disposal, show conclusively that all belong to but one speeies. I have never seen the shell from any but the above quoted locality. 


\section{CARDIUM, L.}

\section{Meeisianum, n. $\mathrm{s}$.}

Pl. 7, Fig. 46.

SHELL resembling C. corbis (Californianum and Nuttallii), but oblique; beaks large, strongly incurved and pointed forwards; anterior end prominent and broadly rounded; posterior end abruptly truncated and very oblique. Surface marked by twenty-two large radiating ribs; these ribs, in the young shell, are acute, becoming rounded as it increases in size, and ultimately becoming distinctly flattened on top; they are crossed by irregular, curved, subsquamose plates, which towards the beaks lose their lamellar character, and are represented by little tubercles; the posterior face of the shell is not costate, or the ribs are represented by only a few indistinct radiating lines; the interspaces between the ribs are narrow and flat, or concave.

Figure, natural size.

Locality: Humboldt County, associated with Callista Voyi, \&c.; collected by Mr. Voy.

From C.corbis, Mart. (which, according to Carpenter, is the same as Californianum and Nuttallii of Conrad), this shell can be distinguished by its form, which is more oblique than the most inequilateral varicties of that species. The ribs are proportionately larger and less numerous, being only about two-thirds as many. The abrupt posterior truncation, and the absence of ribs on that part, are also well-marked characters.

I take pleasure in dedicating this fine species to my friend, Mr. F. B. Meek, of Washington, D. C.

\section{CONCHOCELE. $N$. Gen.}

SHell irregularly quadrate, very inequilateral; a sharp angular ridge passes from the beaks to the posterior end, between which ridge and the cardinal margin the surface is suddenly sunken, presenting the appearance of an offset, the truncation being at nearly a right angle, beyond which the surface retains the same 
general curve as the rest of the shell; ligament cxtcrnal. Hinge composed of a single, long, sharp tooth, running from the beaks, parallel with the cardinal margin, almost to the posterior end. Pallial margin simple.

This shell appears to belong to the family of Isocardiida, presenting some analogies to the genera Edmondia, Unicardium, and Cardiomorpha.

\section{DISJUNCTA, n. s.}

Pl. 7, Fig. 48, $48 a, 48 b$.

Shell subquadrate; beaks terminal, anterior; anterior end abruptly and angularly truncated; base broadly rounded; cardinal margin arched, sloping downwards towards the posterior end. Surface marked only by lines of growth, except near the postcrior part where the peculiar truncation takes place, the surface suddenly descending at a right angle to the curve of the shell, for a short distance, and then resuming its former direction.

Figures, natural size; $48 a$, an outline from above; $48 b$, hinge.

Locality: From Dead Man's Island, near San Pedro Bay; from a hard sandstone assoeiated with two or three previously known Tertiary Fossils. Probably Mioeene.

I found a shell, abundant at Astoria, probably belonging to this genus. It was deseribed by Mr. Conrad in the Wilkes' Expedition Report, p. 724, pl. 17, fig. 10, as Yenus bisecta, and in the Ameriean Journal of Conchology, vol. 1, p. 153, as Cyprina bisecta.

\section{LUCINA, Brug.}

\section{Subgen. HERE, Gabb.}

SHell having all of the usual characters of Lucina, except that the lunule is very decply excavated, penctrating the hinge-plate, and almost perforating it; bounded anteriorly by the anterior lateral tooth, and posteriorly by the cardinal teeth.

This form is very strongly charaeterized, and seems to form a well-marked group in the genus. The following speeies exhibits the character more strongly 
than any prcriously known. Two other specics, both living on the Pacific coast, may also be included in the sub-genus; viz., $L$. excavata, Carpenter, Mazatlan Catalogue, p. 98, and a small subglobose species, with five or six large, rounded, somewhat curved ribs; found in the Gulf of California.

\title{
L. (H.) Richthofeni, n. s.
}

Pl. 8, Fig. 49, $a, b$.

SheLL subglobose, nearly equilateral; beaks small, inclined forwards; margins regularly rounded; a more or less distinctly marked groove passes from the beaks to the posterior margin. Surface marked by numerous, more or less regular, distinct, rounded ribs.

Figure 49, magnified; $49 a$, natural size, view of anterior end ; $49 b$, magnified view of the hinge, left valve.

Locality: San Fernando Valley, north of Los Angeles; Pliocene. Collected by Baron Richthofen.

This species can be at once distinguished by its thick shcll and nearly globular form, from all of the other California Lucinidce. Externally, it is almost identical with $L$. Domingensis in some of its varieties.

\section{CRASSATELLA, Lam.}

\author{
C. Collina. \\ Pl. 8, Fig. 50. \\ $\therefore$ collina, Con.; Pacific R. R. Rep., V. 7, p. 193, pl. 6, fig. 1, 2.)
}

SHELL large, compressed, variably subtriangular; beaks a little in advance of the centre; prominent; anterior end broadly and very slightly excavated above, prominent and rounded below; posterior end sloping sinuously, subangular below; base deeply convex; surface marked by irregular lines of growth; lunule deeply impressed; hinge robust.

Figure, natural size.

Locality and position: Miocenc, from the Santa Iñez Mountains.

The figure in the Pacific Railroad Report is scarcely recognizable, but with the 
assistance of the description, and from the fset that my specimens came from the original loeality from which the first spccimens were obtained, and this is the only species with which I am acquainted that is found there, therc is but little doubt but that my reference of this form to Mr. Conrad's name, is correct.

\title{
MYTILUS, L.
}

\section{Mathewsonir, n. s.}

\author{
Pl. 8, Fig. 51 .
}

SHELl very large, thiek, curved, width and thickness about equal in the centre, flatter towards the base; bcaks terminal, blunt. Surface marked only by lines of growth and irregular concentric undulations.

Figure, natural size. Some speeimens have been found an inch or two longer.

Locality and position: From the Mioeene, south of Martiñez, where it was found, not rare, by Mr. Mathewson and Dr. Fish.

Resembling $M I$. Californiana in its unusually large size, it differs in being less flabellate, more eurved, more convex, and in having nonc of the radiating ribs eharactcristic of that species.

\section{MODIOLA, Lam.}

M. multiradiata, n. s.

P1. 8, Fig. 52.

Snelu moderate in size, variable in form, more or less curved; cardinal margin arched; posterior curved and deseending more or less abruptly; anterior margin sinuated, more or less deeply excavated; beaks very small, subterminal. Surfaee marked by numerous fine radiating lines cxcept on the anterior fourth, whieh is only marked by lines of growth.

Figure, natural size.

Localitzes and position: From the Miocenc at San Emidio, west of Fort Tejon; and at Walnut Creek and Martiñez; Contra Costa County. 


\title{
ARCA, Lam.
}

A. sulcicosta, n.. .

Pl. 9, Fig. 53, 53 a.

SHELL thin, broad; beaks prominent, incarved, approximate, slightly twisted anteriorly; hinge line short; ends and base pretty regularly rounded, posterior basal portion a little the most prominent; area very narrow, slightly sunken. Surface marked by about twenty-five prominent square ribs, with flat, equal interspaces; these ribs are each marked by a more or less distinct median groove, and crossed by pretty strong concentric lines of growth, breaking up the surface into a beautiful beading. Hinge straight, composed of numerous fine teeth, very small and irregular in the middle, larger and slightly oblique towards the ends.

Figure 53, natural size; fig. $53 a$, a magnified view of a few ribs on the anterior portion, to show the details of sculpture.

Locality and position: Found with Dosinia Staleyi, on Mark West Creek, Sonoma County, by Mr. V. S. Staley.

This species differs from $A$. canalis, Con., in being less ventricose, less prominent posteriorly, more produced in front, deeper from beaks to base, in being much thinner, and in the beading of the ribs.

\section{YOLDIA, Möller.}

\author{
Y. COOPERII. \\ Pl. 9, Fig. 54.
}

(Y. Cooperii, Gabb; Proc. Cal. Academy, 1865, p. 189.)

SHELl thin, subcompressed, very inequilateral; beaks minutely placed in advance of the middle, becoming more anterior as the shell increases in size; anterior end narrow, subacuminate; posterior end вгоadly rounded; base most prominent behind the middle, curving upwards to the anterior end. Surface sculptured 
by numerous small concentric ribs, rarely dichotomous, abrupt on the upper side, and sloping downwards on the side towards the base. Muscular scar's large, the posterior a third the largest, broadly suboval, anterior triangular.

Figure, natural size.

Localities: From the Miocene, south of Martiñez, and north of Walnut Creek, Contra Costa County, and a specimen in the collection of the Academy of Natural Sciences, labelled "Oregon," probably Astoria. This species has not been found in the more recent Tertiary formation, though a fresh valve was picked up by Dr. Cooper on the beach at Santa Cruz. In this specimen the epidermis is of a bright olive-green and shining.

The shell varies considerably in outline according to its age. In young shells the beaks are about central; but in the adults, the posterior end increases so much more rapidly than the anterior, that the beaks arc thrown forward so as to be barely more than a third of the width from the anterior extremity.

\section{PECTEN, Brug.}

\section{P. Cerrosensis, n. s.}

Pl. 9, Fig. 55, 55 a.

Shell equivalve, subcircular, broader than long, convex; beaks small; sides sloping concavcly above, rounded below; ears small, subequal, roughened and irregular, sinus very small. Surface marked by eighteen or twenty flat ribs, with flat or slightly concave interspaces; margins undulated, the ends of the ribs being deeply emarginated, and the interspaces being prolonged into tongue-like processes.

Figure, one-half natural sịze (lincar).

Locality: Cerros Island, off the coast of Lower California: probably Miocene. Collected by Dr. J. A. Veateh.

\section{P. Veatchil, n. s.}

Pl. 10, Fig. 56.

Suelu large, equivalve, a very little broader than long; base regularly rounded; sides sloping abovc, with a slight concavity; 
ears unequal; sinus in the right ear of the lower valve moderately deep; surface of ears covered by small radiating ribs and fine imbricating lines of growth; surface of valves ornamented by about eight broad, flat, subnodose ribs, arranged in pairs, and a few small linear ribs on the side; besides the ribs, the whole surface is covered by small radiating lines, and crossed by very irregular lines of growth, and very fine imbricating concentric lines.

Figure, natural size.

Locality: With the preceding.

\section{OSTREA, Linn.}

O. BourgeoISII.

Pl. 11, Fig. 57, 57 a.

(O. Bourgeoisï, Rémond; Proc. Cal. Acad., 1863, p. 13.)

SHell thin, irregularly oval, compressed and contorted near the beaks. Surface marked by a few irregularly squamose lines and faint radiating ridges. Hinge long oblique; ligament pit deep; muscular scar large, not very strongly marked.

Figures, natural size.

Locality and position: Near Kirker's Pass, Contra Costa County; from the Pliocene.

\section{O. ATwOodr, n. 8.}

Pl. 10, Fig. 58, $58 a$, and Pl. 11, Fig. $58 b$.

ShelL broad, irregular, thin; partly attached, sometimes by nearly the whole of the lower surface; free surface of lower valve marked by numerous irregular radiating ribs crossed by lines of growth; upper valves more squamose and not radiated (in the only specimen I have seen). Hinge broad at the base, PAL. VOL. II. -6 
triangular, not deep, and sometimes slightly oblique; inner margin of the shell not denticulated. Muscular scar broad.

Figures, natural size. 58 and $58 a$, lower valves; $58 b$, an upper valve found associated with the others, and apparently bclonging to the same species.

Locality: On San Lorenzo Crcek, Monterey County; cither Miocene or Pliosene. Collected by Mr. M. Atwood.

This shell rcsembles sonewhat the $O$. conchaphila from the coast, but differs in the style of sculpture, the shape of the hinge, and the absence of the internal denticulations.

\section{O. Taylorlana, n. s.}

Pl. 12, Fig. 60, $60 a$.

SHeLl large, thick, irregularly subtriangular, resembling somewhat some of the more massive forms of $O$. Virginica; not attached. Surface covered by heavy, irregular, subsquamose plates, showing more or less of a faint undulation on their edges. Interior unknown. Hinge broad and short(?).

Figures, natural size.

Locality and position: From the Miocene; from San Marcos Pass, near Santa Barbara. Collected by $\Lambda$. S. Taylor.

\section{O. Veatchit, n. s.}

Pl. 11, Fig. 59.

Shelc large, subequivalve, varying from nearly equilateral to very oblique, the obliquity being always to the left side. Surface marked by ten or a dozen large, angular, radiating ribs, some of which arise at or near the beaks, the others branching from the first, or interpolated between them; the interspaces are angular, and the ribs are marked by more or less squamose plates, and occasionally these plates assume almost the character of spines; internally the ribs show only towards the margins. Hinge short, very broad and shallow; no crenulations or denticu- 
lations near the margin. Muscular scar large, suboval to subquadrate.

Figure, natural size of a small and very equilateral specimen. The largest specimen found is about six inches long.

Locality: Cerros Island, associated with Pecten Veatchii, P. Cerrosensis, and the following species. Dr. J. A. Veatch.

O. Cerrosensis, n. s.

Pl. 11, Fig. 61.

SHeLl small, subequivalve, very oblique, strongly falcate, variable in form, upper edge entire, concave; lower margin very strongly undulated, each valve bearing four or five rounded, tongue-like processes, which alternate in the two valves; the margin, on each valve, between these processes, is very much thickened, and presents the squamose edges of successive layers of growth. Surface smooth, or marked only by rather indistinct lines of growth. Hinge long and very oblique, slightly curved. Muscular scars small, rounded subtriangular.

Figure, natural size.

Locality: With the preceding species.

\section{TEREBRATELLA, d'Orb.}

\section{T. Whithey, $\mathrm{n} . \mathrm{s}$.}

Pl. 12, Fig. 62, $62 a$.

SHeLL subcircular, convexity of the two valves about equal, moderate. Lower valve, beak high, area narrow, high, and flat; foramen large, triangular. Upper margin of upper valve nearly straight, sloping slightly from the beak; margins of the shell regularly rounded. Surface marked by about thirty to thirtyfive small, angular, radiating ribs, with angular interspaces; no median sulcus. 
Figures, natural size.

Localities and position: From the Miocene, one mile east of the Excelsior Mine, Napa County, Prof. W.hitney ; and twenty miles east of Clear Lake, on the road from Colusa to the Hot Sulphur Springs; Dr. George.

\title{
MORRISIA, Davidson.
}

\author{
M. Hornit.
}

Pl. 12, Fig. 63.

(M. Hornii, Gabb; Proc. Phila. Academy, 1861, p. 371.)

SHell minute, subcircular, flattened; beak acute; area narrow, long; a faint sinus usually occupies the middle of the lower portion of the upper valve; foramen large, acute above, and rounded or subaugular in the upper valve. Surface, under a lens, presents a minutely granular appearance.

Figure, highly magnified. Length about .11 inch.

Locality and position: From the Post-Pliocene marl at Santa Barbara; collected by Dr. A. L. Heermann. This shell seemed to be abundant in the marl collected by Dr. Heermann; but I have never seen it among the shells collected by the Survey in that vicinity or elsewhere, and failed to find it myself, on subsequently visiting the locality.

\section{ECHINODERMATA.}

\section{CLYPEASTER, Lam.}

\author{
C. GabBII.
}

Pl. 12, Fig. 64, 64 a.

(C. Gabbii, Rémond; Proc. Cal. Acad., 1863, p. 53.)

\section{ECHINARACHNIUS, Van Phels.}

\author{
E. Brewerianus. \\ Pl. 12, Fig. 65, 65 a.
}

(E. Brewerianus, Rémond ; Proc. Cal. Acad., 1863, p. 53.) 


\title{
SCUTELLA, Lam.
}

S. Gibisur.

Pl. 13, Fig. 66, 66 a.

(S. Gibbsii, Rémond; Proc. Cal. Acad., 1863, p. 13.)

\section{ASTRODAPSIS, Con.}

\author{
A. Whitneyi. \\ Pl. 13, Fig. 67, $67 a$. \\ (A. Whitneyi, Rémond ; Proc. Cal. Acad., 1863, p. 52.) \\ A. TUMIDUs. \\ Pl. 13, Fig. 68, 68 a. \\ (A. tumidus, Rémond; Proc. Cal. Acad., 1863, p. 52.)
}

\section{A S T E R I A D A.}

\section{ASTERIAS, L.}

\section{A. Rémondi, n. s.}

Pl. 13, Fig. 69.

Arimal large, robust, five rayed; rays more than twice as long as the diameter of the disk, thick on the margin. Upper surface covered by short, club-shaped spines, narrow towards the base, and bluntly rounded above; on the sides these same spines are more closely placed, and on the under surface they are so close 
that they are in contact; ambulacræ broad, bearing numerous small, very slender spines.

Fig. 67, a view of an average sized ray, natural size.

Locality and position: Found abundantly at "Star Fish Point," on the Pinole Ranch; south of the Straits of Carquinez, about five miles from Martiñez; Miocene.

This species was first discovered by M. Rémond, and is the only fossil star fish yet found in California. At the one locality above it is very abundant, but has never been found elsewhere. It resembles, in its general appearance, $A$. ochracea, Brandt, but the rays are more slender, it wants cntirely the reticulated disposition of the spines; on the upper surfacc, the spincs are larger, of a different form, and much mcre numerous. I have not seen a specimen retaining the disk and a whole ray so as to show the cxact proportion of the two to eaeh other; but by a comparison of a large series, the relative sizes of the parts given above seem to be, at least, very neurlv correct. 


\title{
PALEONTOLOGY OF CALIFORNIA.
}

\author{
V 0 L. II.
}

SECTION I.

TERTIARY IN VERTEBRATE FOSSILS.

P A R T I I.

DESCRIPTION OF NEW SPECIES.

(CONTINUED.) 

The Tertiaries of California, although extensively developed, and usually highly fossiliferous, are of such a character that the included organic remains are rarely well preserved. No Eocene has ever been detected in the State; the Miocene fossils are alnost invariably in bad condition; and it is only from the later deposits that good specimens have been obtained, unless in a few exceptional cases.

As a consequence of this state of things, but few new species have been found in these formations. Among about three hundred recognized species, less than a fifth are new. The majority of them are referable to living forms, while but a few can be with certainty recognized as having been previously described in the reports of Wilkes' Exploring Expedition, or in those of the Pacific Railroad Explorations. The following are all that have been collected since the preparation of the manuscript of the Palæontology of California, Vol. 2, Section 1, Part 1. Appended is a list of the species recognized during the progress of the Geological Survey, with the necessary synonymy. 



\title{
TERTIARY INVERTEBRATE FOSSILS.
}

\author{
( CONTINUED.)
}

MURICIDEA, Swains.

M. (? Phyllonotus) paucivaricata, n. s.

Pl. 14, Fig. 1.

SHell moderate in size, robust, fusiform, spire nearly as long as the aperture; whorls seven, subangulated, sloping above and bearing eight or nine large longitudinal ribs which develop into prominent nodes on the angle; on the body whorl these ribs disappear on the middle or towards the anterior part of the shell. A perture subovate above, narrowed anteriorly, canal slightly deflected and often closed by a union of the two lips; labrum thickened behind, acute on the margin, coarsely dentate internally, and bearing a small compressed tooth a little below the middle, inner lip incrusted by a smooth plate; umbilicus subperforate. Surface marked by two or three varices, acute on the margins, never prominent, and not infrequently entirely obsolete; these varices are usually coincident with the longitudinal ribs; crossing them are from five to seven revolving ribs, between each pair of which are six to eight smaller rounded ribs with acute interspaces, giving the whole shell $a$ closely costate surface; in well-preserved specimens the revolving ribs are crossed by small squamose plates, the remains of the lines of growth.

Length $1.8 \mathrm{inch}$, width $1.05 \mathrm{inch}$, length of aperture $1.1 \mathrm{inch}$.

Locality: Post-Pliocene; Santa Barbara, San Pedro, and San Diego. Not known in a recent state. 
In this shell there is a combination of characters which allies it to two of the genera of the Murices. The nearly obsolete varices, and their small number, with the closed, tubular canal, would place it in Muricidea, but the tooth on the lip, still preserved on the varices, is the character on which Conrad founded Cerastoma, antedated, however, by Phyllonotus of Swainson, who used the numerous varices as the leading character of his genus.

\section{TROPHON, Montf.}

T. squamulifer, Cpr. (in lit.) n. s.

"T. satis turritâ, pallide rufofuscâ; anfr. nucl.? norm. iv tabulatis, tumentioribus, suturis ad angulum $80^{\circ}-100^{\circ}$ acute impressis; costis rad. $\nabla i-\nabla i i$ vix varicosis, angustis, ad angulum posticum interdum spinosis, ad basim continuis; liris spiralibus primum iii fenestratis dein creberrimis, asperis, squamulatis, interstitiis angustis, plus minusve incisis, canali longiore, rectiore, duabus trientibus aperturae aequante, vix apertâ; apertura ovali, labro incrassato, intus circ. v dentati; labio lævi, crassiore.

“Long. .9, long. spir. .35, lat. .51, div. 66.

"Hab. Living; Catalina Isd.; Post-Pliocene, Sta. Barbara.

"With the general aspect of $T$. tenuisculptus, it is at once recognized by the difference in sculpture. The fossil (unique) specimen is much finer than the immature living one sent by Dr. Cooper. In this the varices are not spinous at the angle, and the spiral sculpture is stronger. The difference, however, does not appear to be specific."

The above description and notes were sent me by Dr. Carpenter, in a lettcr, and were bascd on a unique fossil specimen found by myself at Santa Barbara, and an immature recent one from Catalina Island, found by Dr. Cooper.

\section{NEPTUNEA, Bolt.}

N. altispira, n. s.

Pl. 14, Fig. 2.

Sueli allied to $N$. decemcostata, Say, sp., but more slender, and with a much higher spire; whorls angular, the upper surface 
sloping concavely upwards to the suture at a considerably broader angle with the side than in Say's species. Surface bearing several large revolving ribs, one on the angle of the whorl; on the anterior part of the shell these ribs degenerate by becoming less elevated, but retain their width; between the larger ribs, are a few very faint revolving lines; no similar sculpture exists on the top of the whorls; the whole surface is crossed by pretty distinct striæ of growth. Aperture as in decemcostata, except that it is narrower.

Length, about 2.8 inch; width, 1.4 inch.

From the Pliocene of Eagle Prairie, Humboldt County.

This shell is closcly allied to the Eastern decemcostata, and another form recently brought by Mr. Harford from Alaska, the Middendorfii, Coopcr=liratus, Mart. Judge Cooper named his species, after a minute comparison of one specimen with 130 of the Atlantic decemcostata; subsequently Dr. Carpenter, after a similar investigation, pronounced them identical. I have compared several of the Alaska shells with a fine suite of Say's species in the Philadelphia Academy's collection, and believc I can see a constant difference, though in minute characters only. The present fossil, however, is much longer, more slender, with a higher spire and narrower mouth than any of the recent shells. The number of revolving ribs is perhaps about the same as in the living forms, but beyond the first two, they lose their elevation, and the interspace is marked by a smaller rib instead of minute lines. Unfortunately the anterior portion of my single specimen is broken so as to prevent as full a comparison of the characters as I could desire.

\section{N. HUMERosa, n. s.}

Pl. 14, Fig. 3.

Shell large, broadly fusiform; spire high; whorls seven or eight convex; body whorl with a rounded shoulder, above which the shell slopes concavely upwards to the suture, which is small and indistinctly marked; no longitudinal ribs, the first two or three whorls show faint nodes, and in some cases the later volutions are wrinkled by irregular growth. Entire surface covered by numerous small, rounded, revolving ribs, with acute interspaces, and sometimes exhibiting a tendency to alternation in 
size. Aperture broad, subovate, acute behind, narrowing regularly in front; canal short, and slightly deflected; outer lip striate internally; inner lip rather heavily incrusted. Umbilicus well marked but closed.

Length about 5 inches. A specimen, with all of the body whorl, hut having lost the spire, measures, from suture to end of canal, $3.5 \mathrm{inch}$, width of hody $2.4 \mathrm{inch}$, length of aperture 2.7 inch. A smaller specimen shows that the total length of the shell is about twice the length of the mouth.

From the Pliocene of San Fernando, near Wiley's, on the San Fernando Pass, Los Angeles County.

A well characterized spccies, having no close relatives among the known fossil or recent forms of this region.

\section{AGASOMA, Gabb. N. Gen.}

Subfusiform, spire low, body whorl long; canal moderately produced and slightly deflected; aperture elongate, labrum simple, labium incrusted with a thin smooth plate; suture bordered as in Clavella.

This genus resembles Clavella, Swainson, in its general form, and in the bordering of the suture by an elevated portion of the succecding whorl. It differs, however, in the very short spire and in the short and slightly curved canal. It is proposed to receive two specics from the California Miocene, hoth of which, unlike the typical Clavellas, are ornamented by revolving ribs and by tubercles.

$$
\begin{gathered}
\text { A. Gravida, G. } \\
\text { (Clavella gravida, Gabb, Pal. Cal., Vol. 2, p. 4, pl. 1, fig. 6.) }
\end{gathered}
$$

The figure quoted ahove, does not exhihit all of the characters of the pillar lip. In all the specimens I then had, this part was covered by matrix which $I$ was unable to remove. Further specimens show that it is curved, though to a less extent than that of $A$. sinuata figured on the same plate.

$$
\text { A. Sinuata, G. }
$$

(Clavella sinuata, Gabb, Pal. Cal., Vol. 2, p. 5, pl. 1, fig. 7.)

A rare shell, only found in the lowcr portion of the Miooene, associated with Mulinea densata, Ostrea Titan, \&c. 
SURCULA, H. \& A. Ad.

S. Truoniana, G.

(Pleurotoma (Surcula) Tryoniana, G., Pal. Cal., V. 2, p. 6, pl. 1, fig. 9.)

This very rare shell is known by hut two specimens. One is that figured as above, from the Post-Pliocene of San Pedro; the other is a dead and very much decayed shell, picked up by me on the beach near San Pedro, but undoubtedly recent.

\section{NASSA, Lam.}

\section{S. G. CESIA, H. \& A. Adams, 1857.}

\section{Schizopyga, Con., 1857.}

In "Genera of Recent Mollusca," H. and A. Adams describe a large number of subgenera of Nassa, among which is the present one. The description reads, "Spire elevated, whorl rugose or cancellated, rounded; inner lip with the callus defined; outer lip thin, simple." This description covers all of the subgeneric characters which belong to either Nassa perpinguis, Hinds, or Buccinum fossatum, Gld. The former species was placed by them correctly, under this head; while the latter, a closely allied species, and one which, in some of its varieties, is difficult to distinguish from some of the varieties of perpinguis, is placed by them under the subgenus Tritia. To add to the confusion, N. Cooperii, Fbs., is also included in Tritia, while mendica, Gld., is called a Niotha. The last two species belong, without question, to the same group, and are so nearly related that their specific difference has been denied more than once, by excellent authorities. With this portion of the question, however, we have nothing to do at present.

The Adams's have defined clearly all of the important characters of the group to which they have given the name; unless we add that, in both the specics in question, the callus on the inner lip is transversely wrinkled. The same year, in Vol. 6, Pacific Railroad Reports, Mr. Conrad described a genus, of which he said, "the above genus is probably related to Cancellaria," and which he defined as follows: "Buccinifornı; columella concave, plicatc; lower part of body volution deeply channelled, the channel emarginating the columella."

It will be seen that the description is based principally on the deep depression formed by the twisting of the anterior part of the shell, a character of common occurrence in a majority of the Nassas, and visible to a greater or less extent in 
every speeies. The most important charaeter, that of the eallus, was entirely lost sight of from the faet that the speeimens werc mutilated, and the author was not even aware of its existence. His name Schizopyga will therefore have to be dropped, and Coesia adopted in its plaee.

For the leading points of synonomy of these two species, see appended list.

\section{FICUS, Bolt.}

F. PYRIFormis, 11. s.

Pl. 14, Fig. 4.

Shell pyriform, body whorls rounded, slightly flattened on the sides, shouldered, the top sloping upward, with nearly a straight line to the suture; whorls four to four and a half, spire low, suture indistinct. Aperture rather narrow, canal moderate, and slightly curved. Surface ornamented by about forty small, distinct revolving ribs, with occasional smallcr ones interposed, all crossed by prominent lines of growth, more closely placed, and not so large as the revolving sculpture.

Length, 2.3 ineh (end of eanal broken); width, 1.2 ineh; height of apex from posterior angle of aperture, .35 ineh.

From the Mioeene, Martinez. Colleeted by Mr. Mathewson.

From the reeent $F$. Dussumieri, its nearest ally, this speeies differs by its slightly higher spire and less slender, regular form, narrowing more suddenly in advance. From the lines of growth, the eanal, whieh is broken in the present specimen, seems to have been shorter than in the living shell.

\section{F. NODIFERUS, n. s.}

Pl. 14, Fig. 5,

SHELL subpyriform, short, robust, subangulated, side and top of body whorl flattened, upper surface nearly straight, and sloping directly upwards to the suture; upper angle of the whorl carrying a series of about ten flattened nodes, in some cases double; below these, about the middle of the whorl, is another series, less 
prominent, each a little more in advance than the corresponding one above, and connected with it by a faint rib, or rather an undulation of the surface; spire low, whorls five, apical angle a little more acute than the superior slope of the last whorl. Surface marked by numerous small revolving ribs, with broad, flat interspaces, in which are usually one or more additional linear ribs, or fine elevated lines, the whole crossed by minute lines of growth. Aperture broad, canal short.

Length (about) 1.9 inch; width, 1.4 inch; height of spirc from internal angle of mouth, . 3 inch.

From the Miocene at Griswold's, betwcen San Juan and New Idria, Monterey County; Pliocene, Wiley's, San Fernando Pass, Los Angeles County.

A very strongly characterized and peculiar specics, quitc rarc. It has never yet been found as recent as the Post-Pliocene. The best prescrved specimen yet obtained, was found by Mr. Frank E. Brown, in the rich fossiliferous Pliocene Sandstones of the San Fernando Pass, at the west end of San Gabriel Mountains.

SINUM, Bolt. 1798.

Sigaretus, Lam. 1799.

\section{S. PLANicostum, n. s.}

Pl. 14, Fig. 6.

SHell oblique, thin, semiglobose; spire small, somewhat elevated; whorls three and a half to four, rapidly increasing in size; suture small but distinct; body whorl very oblique, ornamented by numerous square revolving ribs with flat interspaces of about equal size; umbilical margin acutely rounded; umbilicus imperforate. The under surface, inside of the margin, not costate, but marked by strong lines of growth. Aperture very large, subelliptical, slightly emarginate by the encroachment of the body whorl; outer lip acute, inner lip slightly thickened.

Length, 1.2 inch; width, 1.3 inch; grentest diameter of mouth, 1 inch; lesser diameter of mouth, .8 inch.

PAL. VOL. II. -8 
Not rare in the Pliocene of San Fernando; collectcd by Mcssrs. Brown, Peckham, and others.

Aceording to the common usage, that of adopting the oldest name since the date of Linn. Syst. Nat. 10 Ed., the name Sinum of Bolton, will have to be used for this genus, having a year of precedence over Sigaretus. It is unfortunate that we are obliged to drop names so familiar as the latter, though, if we do not follow some such rigid rule, it is impossible to tell wherc the diversity of opinions will take authors in the ehoiee of generic names. Klein's name of Catinus, which has been used by Adams, Conrad, and others, dates back to 1753, but there arc other older names, and a growing tendency is devcloping itself to usc the oldest name that can be ferreted out, even if the author was not strietly binominal.

\section{CANCELLARIA, Lam.}

C. gracilior, Cpr. (in lit.) n. s.

"C. t. elongatâ, gracili, clegantissimâ; anfi. nucleosis ii, majoribus, valde declivibus, lavibus, apice immerso; norm. v tumentibus, superne vix subangulatis, suturis valde impressis; costis radiantibus $\mathrm{x}$ rotundatis, ad basim obsoletis, interstitiis undatis, majoribus; lirulis spiralibus exiguis, subdistantibus, supra costes et interstitia eleganter transeuntibus, postice ii minoribus, in medio primum iii, postea vi crebrioribus, circa basim attenuatam vi lineis distantibus; apcrturâ subquadratâ, anticc dilatatâ; labro acuto; labio inconspicuo; columello plicis ii minoribus, declivibus, antice tortâ, plicæ tertiæ simulante, sed vix cmarginata."

"Long. .35, long. spir. .21, lat. .16, div. 38०."

"Hab. Santa Barbara, Post-Pliocene. Of this extremely elegant species, two Epecimens were found by Mr. Gabb, of which one is very perfect. It greatly resembles C. modesta from Neeah Bay, but is mueh more slender. The nucleus is chrysodermoid, very slanting, with sunken spire."

\section{Altispira, n. s.}

Pl. 14, Fig. 7.

SHeLl thick, robust, broadly fusiform; spire high, nearly as long as the aperture; whorls six or seven (?,-apex broken), angu- 
lated, sides flattened, upper surface sloping; suture distinet, impressed. Surface ornamented by about a dozen small angular nodes on the upper angle of the whorl, from which faint longitudinal ribs descend over the body, becoming obsolete about the middle; above similar ribs continue to near the suture; on the earlier whorls these ribs are proportionately much larger; crossing these are three or four elevated revolving striae above the angle, and about twenty below; these alternate pretty regularly in size, eleven or twelve of them being large, the others much smaller; lines of growth faint in the middle, more distinct towards the two ends of the body volution. Aperture moderate in size, broadest in the middle; outer lip simple; inner lip rather heavily incrusted, bearing two strong, rather distant folds.

Length, 2.3 inch ; width, 1.2 inch; length of aperture, $1.3 \mathrm{inch}$; width, .55 inch.

From the Pliocene of San Fernando Pass. Coll. by Mr. Frank Brown-a single specimen.

\section{TROCHITA, Schum.}

T. INORNATA, n. s.

Pl. 14, Fig. 8, 8 a.

Shell low, irregular, slightly oblique, nearly circular; volutions about two to two and a half; suture linear, indistinet; surface entirely without ornament, bearing only a few lines of growth; margin of internal plate sinuous.

Diameter, 1.1 inch; height, .6 inch.

From the Upper Miocene, near Half Moon Bay.

\section{ACMAA, Esch.}

A. RUDIS, n. s.

Pl. 14, Fig. 9, 9a.

Shell moderately large, subelliptical, outline slightly undulated, not distinctly emarginate; apex subcentral, prominent, 
very slightly produced and pointed to one side in the axis of the shorter diameter of the shell. Surface irregularly rugose, the plications or undulations showing a tendency to radiation, though faintly.

Longest diamcter, 1.6 inch; shortest, 1.3 inch; height, . 35 inch.

A single specimen from the Pliocene of San Fernando, near Wiley's.

Although I have but a single specimen of this shell, and st belonging to a genus in which specific characters are usually difficult to definc, I tecl warranted in naming it on account of its marked dissimilarity from all the other known species on the coast. It approaches most nearly in size Lottia gigantea, but the central and more clevated apcx will at once distinguish it ; from Acmoa patina, pelta and scabra, it can be known by the more elliptical form, undulated surface, more clevated apex and irregular outlines, while from $A$. persona and spectrum, its central apex, thrown to one side, instead of in advance, the absence of the strongly marked regular radiating ribs, and its larger sizc, separate it. How far the specific value of all the claracters given in the description will extend, cannot be definitely ascertained cxcept by an examination of more specimens. It is not improbable that the irregular outline and the undulating ribs on the surface may be due to a great extent to the form of the surface to which the shcll was attached during life. It is well known that these shells, like most other forms which attach themselves to one spot, often borrow the jattern of the surface on which they grow. This is often shown by barnacles, cryptas, and anomias; the present genus rarely going beyond a mere distortion of its normal form, or the assumption of a few abnormal undulations. When the surface to which the young animal fixes itself is too rough, it not unfrequently smooths a spot corresponding in size witl the exposed portion of the animal. This I have observed in the case of Acmox persona and A. spectrum at Santa Barbara, where, growing on a coarsc-grained metamorphic sandstonc, almost every shcll fits into a little pit, barely more than a line in depth, level at the bottom, and corresponding cxactly to the form of the shell.

\section{ZIRPHÆA, Leach.}

\section{Z. Gabbir, Tryon.}

\section{Pl. 15, Fig. 10.}

(Zirphaca Gabbii, T., Proc. Phil. Aead., 1866, p. 144, pl. 1, fig. 1.)

This shell was described by Mr. Tryon from a single dead valve, sent to lim by me from San Francisco. I obtained it from a miscellaneous collection of shclls 
from Japan, and have no reason to doubt but that the speeimen eame from Japan with the eolleetion. Mr. Tryon has long held the belief that it was mixed with the otliers by aeeident, and that it was a California speeimen. However that may be, I eannot tell; and we have, at present, no means of settling the question. The speeies is found in California in both a reeent and fossil state, large valves being quite eommon in the Post-Plioeene of San Pedro; nevertheless I do not eonsider this any proof that it may not, likewise, be found living in Japan.

The individual figured is quite young, and is reeent. It was kindly given to me by Mr. R. E. C. Stearns of San Franeiseo.

\section{SILIQUARIA, Schum.}

\section{? S. EDENTULA, n. S.}

Pl. 15, Fig. 11.

Shell moderately large, thin, flattened, elongated sub-elliptical, nearly equilateral; beaks minute, a little posterior to the middle, projecting almost insensibly beyond the cardinal line; cardinal margin sloping slightly and perfectly straight towards the two ends; anterior end convexly and very obliquely subtruncate above, produced and rounded below; posterior end broadly and regularly rounded, a little less prominent below than above the middle; basal margin nearly straight. Surface nearly smooth in the middle, marked by pretty distinct lines of growth towards the ends, especially above.

Length from beak to base, 1.3 ineh; width, 2.6 ineh; beak to anterior end, 1.45 ineh. From the Plioeene of San Fernando.

On exposing the hinge of this shell, I was unable to get at the eharaeters in a perfeetly satisfaetory manner, owing to its extreme thinness and delieaey. The teeth, if they exist, are very small, and seem to be almost entirely obsolete. It is possible that further examination will develop some eharaeters of the hinge whieh will remove it entirely from the above genus, but I preferred plaeing it here provisionally, where it seems most nearly related, to proposing a new generie name on insuffieient grounds. 


\title{
CLIDOPHORA, Cpr.
}

\author{
C. punctata, Con. sp.
}

Pl. 15, Fig. 12.

(Pandora punctata, Con. Jour. Phil. Acad., 1 S. Vol. 7, p. 228, pl. 17, fig. 1.) (Clidophora punctata, Cpr. Proc. Zool. Soe., Lond., 1864, p. 598.)

A shell having all the characters of this species in size, form, \&c., exeept the punctations, was found by Mr. Samuel Peckham in the Upper Miocene bituminous shale, in the "Wheeler Cañon," near San Buenaventura. The valves are in contact, but the inner layer is in part exposed. The closest scrutiny on the outside of this layer fails to disclose the pits considered so characteristic of Mr. Conrad's species; nor do they show themselves on the marginal one-quarter of an inch of the inner surface which $I$ have succeeded in uncovering.

\section{IIEMIMACTRA, Swains.}

\section{? II. occidentalis, n. s.}

Pl. 15, Fig. 13, $13 a$.

SiIELL thin, subovate, inequilateral; beaks small, elosely approximating, plaeed about two-fifths of the length from the anterior end, which is broadly exeavated above, and prominently, though narrowly rounded below; posterior end eonvexly and obliquely sub-truneated; eardinal margin sloping and nearly straight; base broadly and regularly eonvex; a moderately distinet angle runs from the beaks to the posterior basal margin. Surface corered by small but moderately prominent and pretty regularly placed lines of growth.

Length, 2.7 inch; width, 2.2 inch; diametcr, 1.2 inch.

From the Miocene south of Martinez. Rare. A single specimen in my collection, obtaincd by Mr. Mathewson.

This shell rescmbles none of the West Coast Mactroids in form. It has not the cxtremely inequilateral and subcircular outline of the Miocene lenticularis, Nob., nor the trigonal shape of all the Standellas. I can only refer it provisionally to 
Hemimactra, since, from the nature of the matrix, I have found it impossible to expose the hinge.

\title{
PSEUDOCARDIUM, Gabb.
}

By an unfortunate coincidence, Mr. Conrad indicated a genus Pseudocardia, of the family Cardiide almost simultaneously with my publication of the above name. My genus was published February, 1866, while Mr. Conrad's appeared in the Journal of Conchology for April, 1866. It is questionable whether a name should stand when it only differs from an older one by the terminal letter, though Mr. C. assures me that he sees no reason for changing it. I merely wish to call attention to the matter here, in order to guard against confusion, which might possibly arise in the future. In this case $\mathrm{Mr}$. Conrad publishes no semblance of a diagnosis, mercly giving a list of species belonging to the proposed genus.

\section{VENUS, L.}

\author{
V. Pertenuis, Gabb. \\ (V. pertenuis, G. Pal. Cal., Vol. 2, p. 22, pl. 5, fig. 37. Note.) \\ (V. Kennerleyi, Rve. ? G. loe. cit.)
}

When first studying this species, I was unacquainted with Reeve's $V$. Kennerleyi, and had not access to his monograph in Icon. Conch. The short notes by Carpenter in the Report of the British Association, were not inapplicable to the shell before me, but were not sufficiently explicit to enable me to satisfy myself as to its difference from Kennerleyi. On looking at the figure in Icon. Conch. I find that there are strong points of difference. $\quad \boldsymbol{V}$. (Mercenaria) Kennerleyi is an elongate, oval, almost quadrate shell, with heavy ribs, pretty regularly placed, and remarkably prominent. It resembles somewhat a Saxidomus in outline, while the prescnt species is a sub-triangular shell, the surface undulated and striate, but without ribs, properly speaking. It is very probable that pertenuis may prove to be a Chione. I have never yet been able to expose the hinge. A large specimen of Merccnaria perlaminosa, Con., now before me, resembles so ncarly Reeve's figure in botll outline and the charactcr of the ribs, that I strongly suspect them of being identical. In the present specimen (see pl. 15, fig. 14), the ribs are not more than half as numerous as in the one figured in Pal. Cal, Vol. 2, fig. 38; the shell is more clongate in its antero-posterior diameter, and the base is much less rounded. On collecting a series of this species at Santa Barbara, where it is not rare, $I$ found that the pres- 
ent form is much more common than the short rounded one previously figured by me. It has a strongly characterized Mercenaria hinge, and is the only species of that genus yet known on the Pacific coast, unless Reeve's and Conrad's species should prove distinct.

CARYATIS, Roem.

C. Barbarensis, n. s.

Pl. 15, Fig. 15, 15 a.

SHeLl rather small, transverse, gibbous, thin, inequilateral; beaks subcentral, small, strongly incurved, umbones prominent; outline broadly excavated under the beaks, the anterior end produced and narrowly rounded; posterior end narrow; basc irregularly roundcd, strongly curving upwards anteriorly; posterior cardinal margin sloping with a slight, but regular curve. Hinge delicate; teeth small. Surface covered by irregular striæ of growtl. Lunule very large, occupying all the space from the beaks to the anterior end, and bordered by an impressed line.

Length, $1.5 \mathrm{inch}$; width, 1.1 inch; height of a single ralve, .4 inch.

The only shell on the coast, to which this has the most remote resemblance is the young of Amiantis callosa (Cytherea id. Con.) It can, however, be at once distinguished by its longer form, narrower at both ends, its greater convexity, its being much more deeply excavated under the bcaks, and by the surface being marked only by lines of growth, instead of concentric ribs.

$\Lambda$ single specimen was found in the Pliocene of Santa Barbara.

\section{MERETRIX, Lam.}

M. Traskit, Con. sp.

(Lutraria Traskii, Con., P. R. R. Rep., Vol. 5, p. 324, pl. 3, fig. 23.)

This shell is peculiar to the white or Bituminous Shales of the Upper Miocene. It was described by Mr. Conrad from casts in this rock. I havc been fortunate enough to see the cast of the hinge of both valves, and find that it is a Venerid, and appears to belong to the above gcnus. It is rather rare, but seems to be most abundant in the immediate vicinity of Monterey. 


\section{DOSINIA, Scopoli.}

\section{Mathewsonit, n. s.}

Pl. 15, Fig. 16.

Shell nearly circular, excavated under the beaks, about the size of $D$. Dunckerii, but of nearly the form of $D$. ponderosa, convex, thin. Beaks small, pointing strongly forward. Surface nearly smooth in the middle, marked by lines of growth which become strongly developed towards the two ends. Lunule large, lanceolate and slightly impressed.

Length, 1.9 inch; width, 1.8 inch; height of one valve, .25 inch.

From the Miocene of Martinez; and at Griswold's, on the road from San Juan to New Idria.

With the outline of Dosinia ponderosa, and the peculiarity of its surface, this shell has about the average size of $D$. Dunckerii. It is more convex than either, much thinner than the first, and wants the rounded anterior prolongation and radiating lines of the othcr. The lunule is proportionally longer and more slender than either. It seems to be peculiar to the Miocene; D. ponderosa appearing, of full size, and for the first time, in the Pliocene. The other species has not been found fossil in California.

TAPES, Megerle.

\section{T. Staleyi, Gabb.}

Pl. 16, Fig. 17, 17 a.

(Dosinia id., G. Pal. Cal., Vol. 2, p. 24.)

(Tapes id., G. Pal. Cal., Vol. 2, pl. 7, fig. 42.)

A single worn specimen, from which this species was originally described, deceived me as to some of its characters. It has a true Tapes hinge, and the surfacc is erossed by very numerous, minute radiating ribs. It is not unfrequently a little larger than the specimen figured; the pallial sinus is very deep, and rather narrow.

The ribs are of the same character as those of $T$. straminea, but are at least four times as numerous as the most finely costate varieties of that species that $I$ have ever seen.

PAL. VOL. II. -9 


\section{SAXIDOMUS, Con.}

S. gibBosus, n. s.

Pl. 16, Fig. 18, $a, b$.

SHell thin, subquadrate, very inequilateral; beaks anterior, nearly terminal; anterior end exeavated above, narrowly rounded below; posterior end eonvexly subtruncated, sometimes a little oblique; base broadly rounded; eardinal margin gently sloping and slightly eonvex. Surfaee marked by small but distinet and somewhat irregular lines of growth. Lunule large, bordered by a very fine impressed line. Hinge, teeth slender.

Dimensions of two cxtreme specimens: 1st. Lengtll, 2.3 inch; width, 1.7 ineh; diameter of two valves, 1.3 inch; $2 d$. Length, 2 inch; width, 1.7 inch; diameter, 1.2 incls.

Common in the Pliocene of Eagle Prairie, Humboldt County.

Resembles in outline $S$. Nuttallii and $S$. gracilis (=aratus), but can be at once distinguished by its smaller size, more gibbous form, smoother surfaee, and thinner shell.

\section{YOLDIA, Möll.}

Y. Nasuta, Gabb.

(Yoldia nasuta, G. Pal. Cal., Vol. 1, p. 216, pl. 32, fig. 287.)

This shell, deseribed from a single speeimen in the Museum of the Cal. Aead. Nat. Sci., has since proven to be undoubtedly Tertiary; and although the matrix is not unlike some of the Miocenc Shalc, underlying the City of Los Angeles, as cut by artesian borings, it scems to me more like the Post-Pliocene deposit near San Pedro. The specimen is labelled "Los Angeles," and its history is entirely unknown. Its lithological eharaeters plaee it outside of all the known Crctaeeous, so that $I$ harc little hesitation in making the unqualified statement above. 


\section{Y. IMPRESSA, Con., sp.}

(Nucula impressa, Con.; Wilkes's Exped., p. 726, pl. 18, fig. 7.)

(Yoldia id., Meek; Check List, Miocene.)

(Y. Cooperii, G.; Proc. Cal. Acad., 1865, p. 189.)

(Id., G.; Pal. Cal., Vol. 2, p. 31, pl. 9, fig. 54.)

(Id., Cooper and Carpenter; Enumerations of W. C. Moll.)

I deseribed this magnifieent species from a single rccent valve, and numerous fossils in all the deposits down to the Miocene; and in doing so I was supported in my opinion of its being a nondescript by no less authorities than Cooper and Carpenter, who had been working for years in the mollusea of California, and by Hanley, who had just completed a monograph of the family. More recently, while preparing the following tablc of synonymy, I have been convinced that Conrad's impressa is merely a young of the same species. My reasons are: first, there is nothing incompatible in the form; second, the sculpture of the surface, so far as figured in the Wilkes's Exped. Report, is the same as that of Cooperii; and third, $I$ have seen an undoubted specimen of my species from the Miocene of Astoria.

\section{PECTEN, Brug.}

\section{P. Peckhami, n. $\mathrm{s}$.}

Pl. 16, Fig. $19 a$.

Sheld small, thin, subcircular, equivalve, or nearly so, slightly inequilateral; ears nearly equal in size. Upper valve, right ear not separated by any distinct line from the remainder of the surface; the corresponding ear of the lower valve has the same character; left ear flat, the swell of the shell commencing with nearly a right angle with its surface; corresponding ear of lower valves produced, separated from the body of the shell by a marked groove and a deep, narrow sinus. Surface marked by numerous small, irregular, concentric undulations, crossed by obsolete wavy radiating lines, which are most distinct on the ears.

From the bituminous shales of the Upper Miocene, on the Ojai Ranch, Santa Barbara County, where numerous specimens were eollected by Mr. S. F. Perkham. 
Also found in the same formation on the shores of San Pablo Bay, west of Murtinez, and elsewhere.

Dimensions of various specimens: Length, .55 inch; width, .52 inch.

$$
\begin{array}{rrr}
\text { “ } & .49 \mathrm{inch} ; \quad \text { " } & .47 \mathrm{inch} \text {. } \\
\text { " } & .6 \mathrm{inch} ; \quad \text { " } & .57 \text { inch. }
\end{array}
$$

A verage depth of valve, .06 to $.07 \mathrm{inch}$.

This shell belongs to the group (genus ?) Camptonectes, Agas., characterized by a lenticular form, closed all round, edentulous hinge, deep byssal sinus, and the minute radiating sculpture. The species is named after Mr. Peckham, in recognition of his valuable services rendered in collecting the fossils found in the vicinity of the Ojai Ranch.

\section{P. Pedroanus, Trask, sp.}

(Plagiostoma Pedroana, Trask ; Proc. Cal. Acad., 1856, p. 86, pl. 3, fig. 1.)

(P. annulata, Trask; loc. cit., p. 86, pl. 3, fig. 2.)

(P. truncata, Trask; loc. cit., p. 86 , pl. 3, fig. 3.)

In 1856, Dr. Trask named threc species of Plagiostoma, giving full descriptions and well-executed figures as above. His specimens are casts of a number of distorted Pectens, perhaps normally a little oblique, but in most cases with the obliquity exaggerated by pressure. They belong, without question, to but one species, and the first name given will have to be retained. I have carefully studied the specimens, and have visited the locality from which they were obtained. The deposit belongs to the bituminous shale of the Upper Miocene, which underlies the wholo or the greater part of the Los Angeles plain, crops out on the beach at San Pedro, under the Post-Pliocene, and has been reached by artesian borings under the city itself.

The species is about an inch in diameter, ears subequal, shell thin, perhaps normally a little oblique, and the shell-substance contains a few concentric undulations or ribs, which are retained in the cast. No specimens show any shell preserved; so that, if there was any surface sculpture, it is, as yet, unknown.

\section{OSTREA, Linn.}

\section{O. Veatchir, Gabb.}

\section{Pl. 17, Fig. 21, $21 a$.}

(O. Veatchii, Gabb ; Pal. Cal., Vol. 2, p. 34, pl. 11, fig. 59.)

This shell was originally described frcm Cerros Islard, whence it was brought by Dr. Veatch. I have since found it, forming whole beds of Post-Pliocenc age, 
near the port of San Bruno and vicinity, north of Loreto, on the east side of the Peninsula of Lower California. At this loeality, most of the speeimens are six inches or more in diameter, very thick, and quite flat, the ribs being much more numerous, and somewhat smaller than in the speeimens from which my original description and figure were taken.

O. Cerrosensis was found associated with it at San Bruno, and I have reeently received from Prof. Raimondi, of Lima, a shell from the Post-Plioeene of Peru, which seems to be identical with the latter species.

\section{TAMIOSOMA, Conrad.}

\section{T. gregaria, Con.}

Pl. 18, Fig. 22, $a, b, c, d$.

(Tamiosoma gregaria, Con.; Proc. Phil. Aead., 1856, p. 315.)

(Tamiosoma gregaria, Con.; P. R. R. Rep., Vol. 6, p. 72, pl. 4, fig. 18.)

(Balanus Estrellanus, Con.; P. R. R. Rep., Vol. 7, p. 195, pl. 8, fig. 1.)

(Radiolites gregamin. Con.; Proc. Phil. Acad., 1864, p. 214.)

This problematical fossil was described by Mr. Conrad almost simultaneously in the Proceedings of the Philada. Aead. Nat. Scienees, and in the six th volume of the Pacific Railroad Reports, as follows: "An elongated tube, apparently entire, porous and cellular throughout its substance; interior filled with numerous regularly disposed vaulted cells, connected by longitudinal slender tubes, funnel-shaped beneath ; aperture rescmbling that of Balanus."

"T. gregaria. Suhquadrangular, elongated, longitudinally furrowed and striate, and having fine, undulated, transverse lines; mouth small, ohlique; upper part of the tube oblique, dceply indented or Balaniform, and coarsely striated longitudinally. Length, eight inches." He further rcmarks : "Growing in clusters, like Balanus. No sutures indicating separate valves; cells very thin plates, convex surface downwards."

From the ahove, it seems eithcr that Mr. Conrad had no opinion as to the relations of this shell ; or from his repeated comparisons with Balanus, that he appearcd to have suspected it of heing a Cirrhipede; and he accidentally redescrihed it as such, in the seventh volume of the P. R. R. Reports.

In 1864 , however, in the course of some "rectifications," he quotes it as a $R a$ diolite, without other rcmarks than that it is characteristic of the Cretaceous of California.

Though since found at a number of localities in the State, it seems to be always rare. I had not the good fortune to encounter it, until in the summer of 1866 , 
when I obtained speeimens from Estrella, whenee Mr. Conrad described his "Balanus Estrellanus," and later, Mr. Gibbes, a surveyor, presented me with a remarkably fine example from the hills north of Buena Vista Lake. The original specimen, figured in Vol. 6, Pacifie R. R. Rep., came from the Santa Margarita Ranch, northeast of San Luis Obispo. These loealities serve to fix its geologieal age beyond question as belonging in the Bituminous Shale, the best-marked member of our Upper Mioeene. This being ascertained, the next point is to determine what are its relations.

To better understand the shell, it may be well to premise by remarking that $\mathrm{Mr}$. Conrad mistook the two ends of his speeimen, and ealled the base, the aperture. The following deseription, in the absenee of more than one species, will have to serve for both generie and specifie purposes, since in so exeeptional a ease it is diffeult to determine which of the minor eharaeters are of only speeifie value.

Shcll (? lower valve) elongated, eup-shaped, growing in elusters, eemented throughout the entire length wherever in eontaet; eross-seetions irregularly rounded or angulated; lower end of eaeh shell eurved outwards, to aeeommodate the inereased diameter of its neighbors; walls of the eup eomposed of (two, or) three layers. (I have not been able to determine finally, whether an outer impervious layer exists, though I think I have detected it in the Estrella speeimens.) The mass of the wall is eomposed of a series of sub-parallel, longitudinal hollow tubes, rudcly and irregularly divided by minute septa; inner layer entire, homo. geneous, and thin. The interior contains a "body ehamber," or large unoccupied spaec, at its widest end, below whieh the cavity is filled up by an indefinite number of very irregular septa, undulated, and presenting a mammillated surfaee upwards. These septa are produeed by lateral prolongations of the inner wall, the "funnelshaped tubes," mentioned in Mr. Conrad's diagnosis, being the portions last formed as the mantle was retractcd. Some of these "tubes" reaeh to the surfaee of the preceding septum, while many of them are merely little stalaetite-like points, and it is doubtful whether any of them are perforate. In the speeimen from which the first description was taken, these ehambers and even the tubes of the outer walls are all hollow. In the others they are filled with erystalline earbunate of lime; and in these latter, the outcr, or "body chambers," are filled with an indurated sand. The outer surface is rudely and longitudinally striate; the striæ are sometimes crossed by abrupt transverse breaks or subsquamose lines.

The thickness of the outer wall is a quartcr of an inch or less, the tubules eomposing it being about .04 ineh in diameter; the inner stratum of the wall is about .01 to .02 ineh in thiekness.

I have used the term "lower valve," beeause I believe this to be a bivalve shell, for the reasons given below. The upper valve, if it existed, has never yct been detected; but, if my view is correet, this need not be wondered at, espeeially sinee fossils are rare in the deposit in whieh the shell belongs; and with all the faeilities 
of the Gcological Survey of the State to help me, I was four years before I could obtain a single speeimen.

I am convinced that Tamiosoma is a genus of Hippuritida, for the following reasons:

1st. The form of the shell, the septate lower portion of its cavity, the longitudinally tubular walls, and the chamber at the top, for the rcception of the animal, are eharacters which arc found combincd only in this family.

$2 d$. Its mode of growth is exactly analogous to that of the gencra IIippurites and Radiolites.

3d. The structure of its walls is identieal with that of Radiolites; differing only in the relative thickness, as compared with the size of the interior cavity. This analogy is carried out even to the extent of the prisms or columns, whieh constitute the greater bulk of the walls, and arc longitudinal and normally hollow, instead of being transverse and solid as in Inoceramus and Pinnc.

4 th. The septate arrangement of the lower part of the interior cavity is analogous to that existing in Radiolites, as demonstrated by Woodward in the ease of R. Mortoni; the septa in that species differing only in being much less numerous, and a little more symmetrieal than in our fossil. (See Woodward, Quart. Jour. Geol. Soc., Lond., Vol. XI, pl. 5, fig. 2.)

The only arguments that $I$ can find against my position are : 1st. This shell is Tertiary, and the Hippuritidce are all Cretaccous. Although this would be doubtless the first objection urged, I can only see in it a reason against a priori arguments on negative grounds, too common in scienee. Because the family has never beretofore been found outside of the Cretaceous formation, is no reason why its vertieal range may not be extended by discoveries now or hereafter.

2d. The thinness of the walls of the shell, as compared with Radiolites, which I have called its nearest ally.

So long as the details of the structure are preserved, this cannot be a valid objection.

3d. No trace has yet been found of ligament insertions, nor of the enormous hinge teeth charaeteristic of the family. But, as yct, no speeimen has been found showing the entire shell, and the thinness of the walls warrants us, by analogy, in predicating a delicate hinge when that portion shall be discovered. 



\title{
PALEONTOLOGY OF CALIFORNIA.
}

\author{
V 0 L. I I.
}

SECTION I.

TERTIARY INVERTEBRATE FOSSILS.

\author{
P A R T I I I. \\ SYNOPSIS OF THE TERTIARY INVERTEBRATE FOSEILS \\ OF CALIFORNIA.
}

PAL. VOL. XY. -10 



\title{
SY N OPSIS
}

OF

\section{THE IN VERTEBRATE FOSSILS}

OF THE

\author{
TERTIARY FORMATIONS OF CALIFORNIA.
}

IN the following synopsis I have included all the species of Tertiary fossils described from the West Coast, which have been encountered during the progress of the Geological Survey, either in California or in the adjoining State of Oregon. This is indispensable, on account of the intimate relations existing between the Tertiaries of the two States.

The species which have been named, but not recognized or rediscovered, are appended as a separate list at the end. Of the latter, some will doubtless yet be encountered, though the great majority seem to be hopelessly lost. In many cases I have been able to discover what was neant by a description in the Pacific Railroad Reports, only by going to the typical locality, and by that familiarity with the forms, which can only be acquired by years of intimate acquaintance and the study of series of specimens such as amateurs rarely have the good fortune to accumulate. With the double advantage of all the facilities that a government commission could afford, and of half a lifetime of acquaintance with the peculiarities of the anthor's method of working, I have failed in many cases to make out what was intended by the meagre descriptions, and illustrations bad beyond precedent, which are found in the Reports of the Pacific Railroad explorations. 
From the length of time that has elapsed sinee the publication of these rolumes, the author of the Tertiary Palæontology, which they eontain, has in most eases forgotten his own speeies, and has repeatedly declared his inability to assist me; the originals of the descriptions are inaeeessible, packed away with the great bulk of the goverument eollections in the ecllars of the Smithsonian Institution, and I bave been obliged to trust almost entirely to my own aequaintanee with the subject, iu unravelling the tangled skein of the synonymy of the West Coast Tertiaries.

Under these eircumstanees, therefore, it will be surprising if I have sueeceded, in every instanee, in eorrecting the names of the species; and, doubtless, differences of opinion will always exist in many eases. I have, however, endeavored eonseientiously to work out the problem before me, and, should my labors provoke criticism, my only reply sliall be-Go thou and do better. In determinatiou of the living forms, I must aeknowledge partieular indebtedness to Dr. J. G. Cooper, of the Survey, and Dr. P. P. Carpenter, of Montreal. The latter gentleman has gone over nearly all of the reeent mollusea eolleeted by the Survey; and West Coast conehologists depend to a great extent on his opinions, based as they are on a most extensive aequaintance with the subjeet. 


\section{CRUSTACEA.}

CATCER, Linn. C. BreWERII, Gabb.

C. Brcwerii, Gabb ; Pal. Cal., Vol. 2, p. 1, pl. 1, fig. 1.

Pliocene, Santa Barbara.

\section{MOLLUSCA.}

ATURIA, Bronn. A. zICZAC, Sby. sp.

A. ziczac, Con.; Jour. Conchology, 1865. No. 1.

Nautilus ziczac, Sby.; Min. Conch., Vol. 1, p. 12, pl. 1.

N. angustatus, Con.; Wilkes's Exped., p. 728, pl. 20, fig. 56.

Pelagus Vanuxemi, Con.; Jour. Acad. Phila., 2d Ser., Vol. 1, p. 130, pl. 14, fig. 5.

Aturia Vanuxemi, Con.; Smithsonian Check List, No. 639.

Miocene, Astoria.

TRIPTERA, Quoy and Gaim. T. clavata, Gabb.

T. clavata, Gabb; Pal. Cal., Vol. 2, p. 1, pl. 1, fig. 2.

Miocenc, near Griswold's, on the road from San Juan to the New Idria mincs, associated with Dosinia Mathewsonii, Arca microdonta, Acila Castrensis, and various other characteristic species.

HURICIDEA, Swains. M. Californica, Hds. sp.

Murex Californicus, Hds.; Proc. Zool. Soc., 1863, p. 128.

Muricidea id., Cpr.; Brit. Assn. Rep., 1863, p. 663.

Post-Pliocenc, Santa Barbara. Living.

M. (Phyllonotus?) paucivaricata, Gabb.

Muricidea (Phyllonotus?) paucivaricata, Gabb ; Pal. Cal., Vol. 2, p. 43, pl. 14, fig. 1.

Post-Pliocene, S. Barbara, S. Pedro, S. Diego.

\section{Barbarensis, Gabb.}

M. Barbarensis, Gabb; Proc. Cal. A. N. S

Post-Pliocene, Santa Barbara. Living. 
PTERonotus, Swains. P. FEstrvus, Mds. sp.

Murex festivus, Iinds; Proe. Zool. Soc., 1843, p. 127.

$I d .$, Hds.; Voyage of the Sulphur, p. 9, pl. 3, fig. 13, 14.

Pteronotus festivus, Cpr.; Brit. Assn. Rep., 1863, p. 663.

Post-Pliocene, S. Diego. Living.

CCIMERRA, Leach. O. interfossa, Cpr.

Ocinebra interfossa, Cpr.; Brit. Assn. Rep., 1863, p. 663.

Post-Pliocene, Santa Barbara. Living.

O. LURIda, Midd. sp.

Tritonium luridum, Midd. ; Mal. Ross., pt. 2, p. 150, pl. 4, fig. 4 and 5.

Ocincbra lurida, Cpr.; Drit. Assn. Rep., 1863, p. 663.

Post-Pliocenc, S. Pedro. Living.

TROPHON, Montf. T. ronderosum, Gabb.

T. ponderosum, Gabb; Pal. Cal., Vol. 2, p. 2, pl. 1, fig. 3.

Miocene, Griswold's and Walnut Creek, Contra Costa Co.; Pliocene, Kirker's

Pass.

T. squamulifer, Cpr.

Id., Cpr.; Pal. Cal., Vol. 2, p. 44.

Post-Plioeene, Sta. Barbara. Living.

T. multicostatus, Esch. sp.

Trophon multicostatus, H. and A. Ad.; Gen. R. Moll., Vol. 1, p. 77.

Murex multicostatus, Esch.; Zool. Atl., p. 11.

Polyplex gracilis, Perry; Conchology, pl. 9, fig. 4.

Post-Pliocene, Santa Barbara. Living.

T. Orpneus, Gld. sp.

Fusus Orpheus, Gld.; Proc. Boston Nat. Hist. Soe., 1849, p. 142.

Id., Gld. ; Wilkes's Lxped., p. 234, pl. 16, fig. 285.

Trophon Orpheus, Cpr.; Brit. Assn. Rep., 1863, p. 663.

Post-Pliocene, Santa Barbara. Living.

T. tenuisculptus, Cpr.

Trophon tenuiseulptus, Cpr.; An. \& Mạg. Naț. Hist., 1866, p. 227.

Post-Pliocene, Santa Barbara. Living, 
FUSUS. F. AmbUstus, Gld.

F. ambustus, Gld.; Mex. and Cal. Shells, p. 12, pl. 14, fig. 18.

Id., Cpr.; Proc. Zool. Soc., 1856, p. 208.

Post-Pliocene, Santa Barbara, S. Pedro. Living.

NEPTUNEA, Bolt. N. recurva, Gabb.

N. reeurra, Gabb ; Pal. Cal., Vol. 2, p. 3, pl. 1, fig. 4.

This shell belongs apparently to the more recent members of the Mioeene, being found near Tomales Bay, Griswold's, El Toro Rancho, and at Foxin's, in Santa Barbara County. At the latter place the beds overlie the bituminous shale, and appear to be almost a connecting link with the Pliocene.

$$
\text { N. rabuxata, Baird, sp. }
$$

Chrysodomus tabulatus, Baird; Proc. Zool. Soc., 1863, p. 66. Chrysodomus tabulatus, Cpr.; Brit. Assn. Rep., 1863, p. 663.

C. tabulatus, Cooper; Survcy Check List.

Pliocene, Santa Barbara, San Fcrnando, and at the Twelvc-mile House, below San Francisco; Post-Pliocene, Santa Barbara, and San Pedro. Living.

$$
\text { N. Altispira, Gabb. }
$$

Id., Gabb ; Pal. Cal., Vol. 2, p. 44, pl. 14, fig. 2. Pliocene, Eagle Prairie, Humboldt County

\section{N. HUMerosa, Gabb.}

Id., Gabb ; Pal. Cal., Vol. 2, p. 45, pl. 14, fig. 3. Pliocene, S. Fernando.

\section{PRISCOFUSUS, Conrad.}

This genus was merely indicated by $\mathbf{M r}$. Conrad in the Journal of Conchology, to accommodate the following species. No diagnosis has ever been published.

\section{P. Geniculus, Con.}

Fusus geniculus, Con.; Wilkes's Exped., p. 728, pl. 20, fig. 3.

Priscofusus id., Con.; Jour. Conch., 1865, p. 150.

Miocene, Astoria. Living.

Note.-Several other species will be found in the list of unrecognized forms 
IMTULA, II. \& A. Ad. M. Remond, Gabb.

M. Remondi, Gabb; Pal. Cal., Vol. 2, p. 3, pl. 1, fig. 5.

Mioeene, Tomales.

agasoma, Gabb. A. Gravida, Gabb.

Clavella gravida, Gabb; Pal. Cal., Vol. 2, p. 4, pl. 1, fig. 6.

Indet, Con.; P. R. R. Rep., Vol. 5, pl. 7, fig. 63.

Agasoma gravida, Gabb; supra, p. 46.

Miocene, near Martinez, and at Ocoya Creek.

\section{A. sinuata, Gabb.}

Clavella sinuata, Gabb; Pal. Cal., Vol. 2, p. 5, pl. 1, fig. 7. Agasoma sinuata; Gabb; supra, p. 46.

Miocene, Walnut Creek, Contra Costa County.

PISANIA, Bivona. P. Fortis, Cpr.

Pisania fortis, Cpr.; An. \& Mag. Nat. Hist., 1866, p. 227.

Post-Pliocene, Santa Barbara.

SURCUlA, II. and A. Ad. S. Carpenteriana, Gabb.

Pleurotoma (Surcula) Carpenteriana, Gabb; Proc. Cal. A. N. S., 1865, p. 183 Id., Gabb ; Pal. Cal., Vol. 2, p. 5, pl. 1, fig. 8.

Plioeene, S. Fernando, Santa Rosa ; Post-Pliocene, Santa Barbara, San Pedro Living.

\section{S. Tryoniana, Gabb.}

P. (S.) Tryoniana, Gabb ; Pal. Cal., Vol. 2, p. 6, pl. 1, fig. 9.

Post-Pliocene, S. Pedro. Living.

\section{DRILLIA, Gray. D. INermis, Hds. Sp.}

Pleurotoma inermis, Hds.; Proc. Zool. Soc., 1843, p. 37.

Id., Hds.; Voy. Sulphur, p. 16, pl. 5, fig. 8.

Drillia inermis, Cpr.; Brit. Assn. Rep., 1863, p. 657.

Post-Pliocene, S. Pedro. Living. 
D. Perversa, Gabb.

Pleurotoma perversa, Gabb; Proc. Cal. A. N. S., 1865, p. 183.

Id., Gabb ; Pal. Cal., vol. 2, p. 6, pl. 1, fig. 10.

Post-Pliocene, Sta. Barbara, S. Pedro. Living.

\section{Voyi, Gabb.}

D. Voyi, Gabb; Pal. Cal., vol. 2, p. 7, pl. 1, fig. 11.

Pliocene, Humboldt County, at Eagle Prairic and Bear R.

Clathurella, Cpr. C. Conradiana, Gabb.

C. Conradiana, Gabb ; Pal. Cal., vol. 2, p. 7, pl. 1, fig. 12.

Post-Pliocene, Santa Barbara. Living.

IANGELIA, Leach. M. VARIEgata, Cpr.

Mangelia variegata, Cpr.; Brit. Assn. Rep., 1863, p. 658

Post-Pliocene, Santa Barbara. Living.

TRITONIUM, Link. (S. G. Priene, H. and A. Ad.) T. (P.) OregONENSIS, Redf. sp.

Triton Oregonensis, Rcdficlã ; A. N. Y. Lyc., vol. 4, p. 165.

Id., Gld.; Wilkes's Exped., p. 241.

Fusus Oregonensis, Rve.; Icon. Conch., No. 61.

F. cancellatus, Rve.; Loc. cit., No. 62.

T. (Priene) Oregonensis, Cpr.; Rep. W. C. Moll., Brit. Assn., p. 583.

Post-Pliocene, Santa Barbara. Living.

RANELLA, Lam. ? R. TRiquetra, Cpr.

R. triquetra, Cpi. ; ("teste Nutt. MSS."), P. Zool. Soc., 1856, p. 227.

Post-Pliocene, San Diego. Living.

R. Californica, Hds.

R. Californica, Hdș.; Ann. Mag. Nat. Hist., vol. 2, p. 255.

R. Californica, Hds.; Voy. Sulphur, p. 12, pl. 2, fig. 4 and 5.

Pliocene, Kirker's Pass ; Post-Pliocene, Santa Barbara, San Pedro. Living. PAL. VOL. II.-11 


\section{R. MathewsoniI, Gabb.}

R. Mathewsonii, Gabb ; Pal. Cal., vol. 2, p. 8, pl. 2, fig. 13. Miocene, near Martinez.

COMINELlA, Gray. (S. G. Amphissa, H. and A. Ad.) C. (A.) corrugata, Rve. sp.

Buccinum corrugatum, Rve.; Icon. Conch., pl. 14, fig. 110.

Truncaria corrugata, Cpr.; Brit. Assn. Rep., 1863, p. 662.

Cominella (Amphissa) cormegata, Cpr.; Brit. Assn. Rep., 1866.

Post-1'liocene, San Pedro, Santa Barbara. Living.

NASSA, Lam. N. Tegula, Rve.

Nassa tegula, Rve.; Icon. Conch., Nassa, No. 98, pl. 15.

Post-Pliocene, Santa Barbara, San Pedro. Living.

\section{N. MeNdiCA, Gld.}

N. mendica, Gld. ; Proc. Bost. N. H. Soc., 1850, p. 155.

N. mendica, Gld.; Wilkes's Exped., p. 263, pl. 19, fig. 331.

Post-Pliveene, Santa Barbara, San Pedro. Living

\section{N. CooperiI, Fbs.}

N. Cooperii, Forbes; Zool. Proc., Lond., 1850, p. 273, pl. 11, fig. 4.

Post-Pliocene, Santa Barbara, San Pedro. Living. Erroneously deseribed as from the Sandwich Islands.

\section{N. Fossata, Gld. sp.}

Buccinum fossatum, Gld.; Proc. B. N. H. Soc., 1850, p. 152.

Nassa id., H. and A. Ad.; Gen. Recent Mollusca.

B. elegans, Rve.; Proc Zool. Soc., 1842.

Schizopyga Californica, Con.; P. R. R. Rep., vol. 6, p. 69, pl. 2, fig. 1.

Mioeene, Martinez, Griswold's, Walnut Creek, Foxin's, Maxima Martinez

Rancho; Pliocene, San Fernando, near San Franeisco, Santa Rosa, and Russian R. Valley; Post-Pliocene, Santa Barbara, San Pedro, and San Diego. Living. 


\section{N. Perpinguis, Hds.}

N. perpinguis, Hds. ; Voy. Sulphur, p. 36, pl. 9, fig. 12, 13.

N. interstriata, Con.; P. R. R. Rep., Vol. 5, p. 327, pl. 6, fig. 49.

Plioeene, San Fernando; Post-Plioeene, San Pedro, Santa Barbara. Living.

PURPURA, Brug. P. saxicola, Val.

P. saxicola, Val.; Voy. Venus, pl. 8, fig. 4.

P. canaliculata, Duel. ; An. N. Sc., 1832, p. 104, pl. 1, fig. 1.

P. ostrina, Gld.; Otia, p. 225.

P. lapillus, Coop.; (not Linn. sp., Lam.), N. Hist. Washn. Terr., p. 372.

P. attenuata, Rive.; Ieon. Coneh., pl. 10, fig. 49.

P. emarginata, Desh.; (not Rve.), Mag. de Zool., 1841, pl. 25.

Plioeene, Santa Rosa, Kirker's Pass, San Fernando; Post-Plioeene, Santa

Barbara. Living.

MONOCERAS, Lam. M. LUGubris, Sby.

Monoceras lugubris, Sby.; Genera, No. 5, fig. 3.

Post-Plioeene, Santa Barbara Island. Living.

\section{M. engonatum, Con.}

M. engonatum, Con.; Jour. Phil. Aead., 1 Ser., Vol. 7, p. 264, pl. 20, fig. 17.

M. unicarinatum, Rve.; Ieon. Coneh., sp. 1.

Post-Plioeene, San Pedro. Living.

CUMA, Humph. C. Biplicata, Gabb.

C. biplicata, Gabb ; Pal. Cal., Vol. 2, p. 9, pl. 2, fig. 14.

Mioeene, Martinez.

OLIVELla, Swains. O. Biplicata, Sby. sp.

Oliva biplicata, S'by.; Tank. Cat., App., p. 33.

Olivella biplicata, Cpr.; B. A. Rep., 1863, p. 661; H. \& A. Ad.; Gen., p. 146.

Post-Plioeene, Santa Barbara, San Pedro, San Diego. Living.

\section{O. Pedroana, Con. sp.}

Strephona Pedroana, Con.; P. R. R. Rep., Vol. 5, p. 327, pl. 6, fig. 51.

Olivella boetica, Cpr.; B. A. Rep., 1863, p. 661.

Post-Plioeene, Santa Barbara, San Pedro. Living. 
APCILLARIA, Lam. A. Fishit, Gabb.

A. Fishii, Gabb ; Pal. Cal., Vol. 2, p. 9, pl. 2, fig. 15.

Miocene, Martinez

VOLUTILITHES, Swains. "V. indurata," Con.

Rostellaria indurata, Con.; Wilkes's Exped., p. 727, pl. 19, fig. 12.

Volutilithes id., Con.; Jour. Coneh., 1865, p. 151.

Miocene, Oregon, Tomales Bay, Cal.; Pliocene, Half-Moon Bay.

This shell is found occasionally, though rarely. I have seen several speeimens, but never in a sufficiently good eondition to determine fully its generic relations. That it is not a Rostellaria, nor in faet one of the Strombida, is sufficiently evident from its form. I lave never becn able to deteet even the rudiments of folds or its eolumella, which fact, together with its noncancellate surface, carry it out of the other genus in whieh Mr. Conrad has placed it. It will probably be found, when we obtain better specimens, to belong to the Fusino.

Columbelia, Lam. C. (Alia) Richthofeni, Gabb.

C. (.1lia) Tiehthofeni, Gabb; Pal. Cal., Vol. 2, p. 10, pl. 2, fig. 16.

Pliocene, Russ. R., San Fernando, and San Franciseo.

AifYCLA, II. \& A. Ad. A. gausapata, Glil. sp.

Columbeila gausapata, frld.; Proc. Bost. N. H. Soc., 1850, p. 170.

Il., Gld.; Wilkes's Exped., p. 267, pl. 19, fig. 337.

1 myela ill., Clr.; Brit. Asn. Rep., 1868, p. 662.

Nassa Pedroanu, Con.; P. R. R. Rep., Vol. 5, p. 327, pl. 6, fig. 48.

Pust-Plioene, Santa Barbara, San Pedro. Living.

A. cartinata, Ids. sp.

Columbella carinata, IIds.; Toy. Sulphur, p. 89, pl 10, fig. 15, 16.

Columbella carinata, Cpr.; ("perhaps Amycla,") Br. A. Rep. 1863, p. 662.

Post-Plioecne, Santa Barbara. Living.

MARGIHELLA, Lam. M. JewetTi, Cpr.

M. Jeuctti, Cpr.; P. Zool. Soc., 1856, p. 207.

Post-Plioeene, San Pedro. Living. 
FICUS, Bolt. F. PYriformis, Gabb.

F. pyriformis, Gabb; Pal. Cal., Vol. 2, p. 48, pl. 14, fig. 4.

Mioeene, Martinez.

\section{F. NODiferus, Gabb.}

F. nodiferus, Gabb ; Pal. Cal., Vol. 2, p. 48, pl. 14, fig. 5.

Mioeene, Griswold's; Plioeene, San Fernando.

NATica, Brug. N. clausi, Brod. \& Sby.

N. clausa, Brod. \& Sby.; Zonl. Beeehey's Voy., p. 136, pl. 34, fig. 3 ; and pl. 37, tig. 6.

Post-Plioeene, Santa Barbara and San Pedro. Living.

LUNATIA, Gray. L. Lewisi, Gld., sp.

Natica Lewisii, Gld.; Proe. Bost. N. Hist. Soe., 1847, p. 239.

N. Lewisii, Gld.; Wilkes's Exped., p. 211, pl. 15, fig. 253.

Natica Herculea, Midd.; Mal. Ross., Part 2, p. 96.

Lunatia Lewisii, Cpr.; B. A. Rep., 1863, p. 661.

Plioeene, Santa Barbara, San Fernando, Kirker's Pass; Post-Plioeene, Santa

Barbara, San Pedro, San Nieholas Isd. Living.

INEVERTA, Risso. N. saxea, Con.

Neverita saxea, Con.; Jour. Coneh., 1865, p. 151.

Natica srixea, Con.; Wilkes's Exped., p. 727, pl. 19, fig. 7.

Mioeene, Oregon.

\section{N. Callosa, Gabb.}

N. callosa, Gabb; Pal. Cal., Vol. 2, p. 10, pl. 2, fig. 17, a, b, e.

Mioeene, Walnut Creek

\section{N. Recluziana, Desh., sp.}

Natica Recluziana; Desh.; Mag. de Zool., 1841, Mollusea, pl. 37.

Neverita Rechuziana, H. \& A. Ad.; Gen. Ree. Moll., Vol. 1, p. 208.

Natica Inezana, Con. ; P. R. R. Rep., Vol. 7, p. 195, pl. 10, fig. 5, 6.

Natica Ocoyana, Con.; P. R. R. Rep., Vol. 5, p. 328, pl. 7, fig. 57.

Mioeene, Santa Inez Mts., Walnut Creek, Pose Creek, Martinez, Death Valley; Plioeene, Santa Barbara, San Fernando; Post-Plioeene, Sunta Barbara, San Pedro. Living. 
SINUM, Bolt. Sigaretus, Lam. S. PLAnicostum, Gabb.

S. planicostum, Gabb. ; Pal. Cal., Vol. 2, p. 49, pl. 14, fig. 6. Plioeene, San Fernando.

SCALARIA, Lam. S. subcoronata, Cpr.

Scalaria subcoronata, Cpr.; B. A. Rep., 1863, p. 660.

Post-Plioeene, Santa Barbara. Living.

S. crebricostata, Cpr.

Scalaria crebricostata, Cpr. ; Loc. cit., p. 660.

Post-Plioeene, San Pedro. Living.

$$
\text { S. Bellastriata, Cpr. }
$$

Scalaria bcllastriata, Cpr. ; Loc. cit., p. 660.

Post-Pliocene, Santa Barbara. Living.

opalia, H. \& A. Ad. O. (crenatoldes, var.) insculpta, Cpr.

Opalia (crenatoides, var.) insculpta, Cpr.; An. Mag. Nat. Hist., 1866, p. 277. Post-Pliocene, Santa Barbara.

MYURELLA, Hds. M. SIMPLEX, Cpr.

Myurella simplex, Cpr.; B. A. Rep., West Coast Moll., 1863, p. 657.

Post-Plioeene, Santa Barbara, San Pedro. Living.

Conus, Linn. C. Californicus, Hds.

C. Californicus, Hinds; Voy. Sulphur, p. 7, pl. 1, fig. 3, 4, 5.

C. ravus, Gld.; Shells of Mex. and Cal., p. 13, pl. 14, fig. 20.

C. ravus, Cpr.; Proc. Zool. Soe., Lond., 1856, p. 206.

Pliocene, San Fernando; Post-Plioeene, San Diego, San Pedro, and Santa Barbara. Living.

CYPREA, Linn. (S. Gen. Luponia, Gray.) C. (L.) spadicea, Swains. Cyproxa spadicea, Swainson; Phil. Mag., Vol. 61, p. 370 ; Zool. Ill., pl. 182. Luponia spadiceu, Gray; Cpr.; B. A. Rep., p. 657.

Post-Plioeene, Santa Barbara Isd. Living. 
CANCELLaria, Lam. C. (Euchia) tritonidea, Gabb.

C. (Euclia) tritonidea, Gabb ; Pal. Cal., Vol. 2, p. 11, pl. 2, fig. 18.

? Pust-Pliocene, San Pcdro.

$$
\text { C. vetusta, Gabb. }
$$

C. vetustr, Gabb ; Pal. Cal., Vol. 2, p. 12, pl. 2, fig. 19.

Miocene, Martincz.

$$
\text { C. GRACilior, Cpr. }
$$

C. gracilior, Cpr.; Pal. Cal., Vol. 2, p. 50.

Post-Pliocene, Santa Barbara.

$$
\text { C. Altispira, Gabb. }
$$

C. altispira, Gabb; Pal. Cal., Vol. 2, p. 50, pl. 14, fig. 7.

Pliocene, San Fernando.

BITTIUI, Leach. B. ASPERUM, Gabb.

Turbonilla aspera, Gabb ; Proc. Phil. Acad., 1861, p. 368.

Bittium asperum, Gabb; Pal. Cal. (1866), Vol. 2, p. 12, pl. 2, fig. 20.

B. asperum, Cpr.; A. Mag. N. Hist., 1866, p. 276.

B. rugatum, Cpr.; Brit. Assn. Rep., 1866, p. 539.

Post-Pliocene, Santa Barbara, San Diego, San Pedro. Living.

B. armiluatum, Cpr.

Bittium armillatum, Cpr.; A. Mag. Nat. Hist., 1866, p. 276.

Post-Pliocene, Santa Barbara. Living.

\section{CERITHIdEA, Swains. C. Californica, Hald.}

Cerithium Californicum, Hald, 1840, Fr. W. Univ. Moll., cover of No. 1.

Cerithidea Californica, Hald, Ms

Cerithium (Potamis) sacratum, Gld.; 1849, Proc. Bost. N. Hist. Soc., Vol. 3, p. 118.

Id., Gld. ; Wilkcs's Exped. Rep., p. 114, pl. 10, fig. 116.

Potamis pullatus, Gld.; P. R. R. Rcp., Vol. 5, p. 333, pl. 11, fig. 23, 24.

Cerithidea sucrata, Cpr. ; 1856, Proc. Zool. Soc., Lond., p. 226.

Mincene, Santa Monica Mis.; Post-Pliocene, San Pedro. Living. 
IFELANIA, Lam. M. TAYLORI, Gabb.

M. Taylori, Gabb ; Pal. Cal., Vol. 2, p. 13, pl. 2, fig. 21.

From a Fresh-water Lertiary Deposit on Snake River, Idaho.

IITHASIA, Hald. l. ANTIQUa, Gabb.

L. antiqua, Gabb; Pal. Cal., Vol. 2, p. 13, pl. 2, fig. 22.

With the preceding.

IITTORINA, Ferr. L. planaxis, Phil.

L. planaxis, Phil.; teste Cpr.; Proc Zool. Soc., Lond., 1856, p. 226.

L. patula, Gld.; 1846, Proc. Bost. N. Hist. Soc., p. 52.

Pliocene, Kirker's Pass ; Post-Pliocene, San Diego, San Nicholas Isd. Living

\section{Rémondi, Gabb.}

L. Rémondii, Gabb; Pal. Cal., Vol. 2, p. 14, pl. 2, fig. 23.

Pliocene, Kirker's Pass.

IACUNA, Turton. L. sOLidula, Loven.

Lacuna solidula, Loven; teste Cpr. ; Brit. Assn. Rep., 1863, p. 656.

L. carinata, Gld.; Proc. Bost. N. H. Soc., 1848, p. 75.

Littorina Pedroana, Con.; P. R. R. Rep., Vol. 5, p. 327, pl. 6, fig. 50.

Modelia striata, Gabb. ; Proc. Phil. Acad., 1861, p. 368.

Post-Pliocene, Santa Barbara, San Pedro. Living.

TURRITELLA, Lam. T. Cooperit, Cpr.

T. Cooperii, Cpr.; Brit. Assn. Rep., 1863, p. 655.

Pliocene, San Fernando; Post-Pliocene, Santa Barbara, San Pedro. Living.

T. Jewettir, Cpr.

Turritella Jewettii, Cpr.; A. Mag. N. H., 1866, 276.

Post-Pliocene, San Diego, Santa Barbara. Living.

\section{T. Hoffmannit, Gabb.}

T. Hoffmannii, Gabb; Pal. Cal., Vcl. 2, p. 14, pl. 2, fig. 24.

Miocene, Santa Clara County; Sespi Cañon, Santa Barbara County. 


\section{T. Inezana, Con.}

T. Inezana, Con.; P. R. R. Rep., Vol. 7, p. 195, pl. 8, fig. 4. Miocene, Santa Inez and Santa Monica Mts.

T. variata, Con.

T. variata, Con.; P. R. R. Rep., Vol. 7, p. 195, pl. 8, fig. 5 .

Miocene, Santa Monica Mts.

\section{CECUM, Fleming. C. CooperiI, Cpr.}

C. Cooperii, Cpr.; Brit. Assn. Rep., 1863, p. 655.

Post-Pliocene, San Diego. Living.

\section{CRUCIBULUM. Schum. C. spinosum, Sby.}

Calyptrcea spinosa, Sby; Gencra of Shells, Sp. 6. Crucibulum spinosum, Rve.; Icon. Conch., Sp. 10.

Crucibulum spinosum, Con.; P. R. R. Rep., Vol. 5, p. 327, pl. 5, flg. 46. Post-Pliocene, Santa Barbara. Living.

TROChita, Schum. T. costellata, Con.

T. costellata, Con.; P. R. R. Rep., Vol. 6, p. 195, pl. 7, fig. 3. Miocene, Sauta Monica and Santa Inez Mts.

\section{T. Filosa, Gabb.}

T. filosa, Gabb; Pal. Cal., Vol. 2, p. 15, pl. 2, fig. 25.

Miocene, Walnut Creek.

T. inornata, Gabb.

T. inornata, Gabb; Pal. Cal., Vol. 2, p. 51, pl. 14, fig. 8, 8 a. Miocene, Half-Moon Bay.

\section{CRYPTA, Humph. C. Grandis, Midd.}

C. grandis, Midd.; Mal. Ross., pt. 2, p. 101, pl. 11, fig. 8, 9, 10.

Crepidula praerupta, Con.; Wilkes's Exped., p. 727, pl. 19, fig. 9, 10.

Crypta praerupta, Con.; Jour. Conch., 1865, p. 151.

PAL. VOL. II. -12 
Crypta princeps, Con.; P. R. R. Rep., Vol. 5, p. 326, pl. 6, fig. 52.

Miocene, Walnut Creek, Martinez, Foxin's, Astoria, Tomales, Kirker's Pass, and elsewhere; Plioeene, San Fernando, Santa Barbara, Half-Moon Bay, Kirker's Pass, Santa Rosa, Russian River; Post-Plioeene, Santa Barbara, San Pedro. Living.

$$
\text { C. lingulata, Gld., sp. }
$$

Crepidula lingulata, Gld.; Otia, p. 15.

Crypta lingulata, H. \& A. Ad.; Gen. Ree. Moll., Vol. 1, p. 369.

Post-Plioeene, Santa Barbara. Living.

$$
\text { C. ADUNCA, Sby., sp. }
$$

Crepidula adunca, Sby.; 'Tank. Cat., app., p. 7.

Crypta adınca, II. \& A. Ad.; Gen. Ree. Moll, Vol. 1, p. 369.

Plioeene, San Fernando; Post-Plioeene, San Pedro. Living.

If adunca, Sby. = rostriformis, Gld., as believed by Carpenter, then this speeies will probably inelude rostralis, Con., from the Oregon Mioeene.

$$
\text { C. Navicelloides, Nutt., sp. }
$$

Crepidula navicelloides, Nutt.; Jay's Cat., 3035.

Crypta id., Cpr.; Brit. Assn. Rep.

Post-Plioeene, Santa Barbara. Living.

$$
\text { C. DoRsata, Brod., sp. }
$$

Crepidula dorsata, Brod.; Zool. Proe., 1834, p. 38.

Crypta dorsata, H. \& A. Ad.; Gen. Ree. Moll., Vol. 1, p. 369.

Plioeene, San Fernando. Living.

HIPPONYX, Defr. H. Grayands, Mke.

H. Grayanus, Mke. ; Proe. Zool. Soe., Lond., 1856, p. 4.

Post-Plioeene, San Pedro. Living.

Phasianella, Lam. P. сомpta, Gld.

P. compta, Gld.; P. R. R. Rep., Vol. 5, p. 333, pl. 11, fig. 25, 26.

P. perforata, Phil.; Zeitseh. f. Mal. 1848, p. 164, (teste Rve.)

Post-Plioeene, San Pedro, San Diego. Living.

Carpenter denies the eorreetness of Reeve's opinion in this ease. 
PACHYPOMA, Gray. P. BIANGULATA, Gabb.

P. biangulata, Gabb: Pal. Cal., Vol. 2, p. 15, pl. 3, fig. 26.

Miocene, Martinez.

$$
\text { P. gibberosum, Chemn, sp. }
$$

Trochus gibberosus, Chemn.; Conch. Cab., Vol. 10, p. 278, vignette 23.

T. inequalis, Gmel. ; (not Martyn), p. 3585.

T. ochraceus, Phil.; Zeits. f. Mal., 1846, p. 101.

Pachypoma gibberosum, Cpr.; Brit. Assn. Rep., 1863, p. 651.

Post-Pliocene, San Pedro. Living.

POMAULAX, Gray. P. undosus, Wood, sp.

Trochus undosus, Wood; Suppl., p. 16, pl. 5, fig. 1.

T. balcenarum, Val.; Voy. Venus, pl. 3, fig. 1.

Pomaulax undosus, Cpr.; B. A. Rep., 1863, p. 651.

Post-Pliocene, San Pedro and San Diego. Living.

Calliostoma, Swains. C. annulatum, Mart., sp.

Trochus annulatus, Mart.; Univ. Conch., Vol. 1, fig. 33.

Zizyphinus annulatus, A. Ad.; Zool. Proc., 1851, p. 164.

Zizyphinus annulatus, Gray; Dieffenbach's N. Zealand, p. 237, No. 72.

Calliostoma annulatum, Cpr.; Brit: Assn. Rep., p. 652.

Post-Pliocene, San Pedro. Living.

$$
\text { C. costatum, Mart., sp. }
$$

Trochus costatus, Mart.; Univ. Conch., pl. 10, fig. 3.

Calliostoma costatum, Cpr.; Brit. Assn. Rep., p. 652.

Pliocene, San Fernando; Post-Pliocene, San Pedro. Living.

\section{C. canaliculatum, Mart., sp.}

Trochus canaliculatus, Mart.; Univ. Conch., Vol. 1, fig. 32.

Zizyphinus canaliculatus, Gray; Travels in New Zealand, p. 237.

Calliostoma canaliculatum, Cpr.; Brit. Assn. Rep., p. 652.

Post-Pliocene, San Pedro. Living. 
C. TRICOLOR, Gabb.

Calliostoma, tricolor, Gabb; Proc. Cal. Acad., 1865, p. 186.

Id., Gabb ; Pal. Cal., Vol. 2, p. 17, pl. 3, fig. 28.

Post-Pliocene, San Pedro, Santa Barbara. Living.

PTYCHOSTYLIS, Gabb. P. CAFFEA, Gabb.

Turcica (P.) caffea, Gabb; Proe. Cal., A. N. S., 1865, p. 187.

$I d .$, Gabb; Pal. Cal., Vol. 2, p. 16, pl. 3, fig. 27.

Thalotia caffea, Coop.; Geog. Cat. W. C. Moll., 1867, p. 26.

Compare Trochus Adamsianus, Schrenck, 1867, Reisen im Amur-Lande, Moll., p. 358 , pl. 16, fig. 5 .

Post-Plioeene, San Pcdro. Living.

Chlorostoma, Swains. C. Aureotinctum, Fbs., sp.

Trochus (Monodonta) aureotinctum, Fbs.; Zool. Proc., 1850, p. 271, pl. 11, fig. 7.

Chlorostoma aureotinctum, Cpr.; Brit. Assn. Rep., 1863, p. 652.

Post-Pliocenc, Santa Barbara. Living.

\section{Pfeifferit, Phil., sp.}

Trochus Pfeifferii, Phil.; Zeits. f. Mal., 1846, p. 104; Monog. Troch., p. 152, pl. 25 , fig. 2.

Trochus marcidus, Gld.; Mex. and Cal. Shells, p. 8, pl. 14, fig. 11.

Chlorostoma Pfeifferii, Cpr.; B. A. Rep., 1863, p. 652.

Post-Pliocene, Santa Barbara Island. Living.

$$
\text { C. Brunneum, Phil., sp. }
$$

Trochus brunneus, Phil.; Zeits., 1848, p. 189 ; Monog. Troch., p. 300, pl. 43, fig. 19.

Chlorostoma brunneum, Cpr.; B A. Rep., 1863, p. 652.

Post-Pliocene, Santa Barbara Island. Living.

$$
\text { C. funebralie, A. Ad. }
$$

Chlorostoma funebrale, A. Ad.; Proc. Zool. Soc., 1854, p. 316.

Chlorostoma funebrale, Cpr.; B. A. Rep., 1863, p. 652.

Post-Pliocene, Santa Barbara, San Pedro. Living. 
TrochISCUS, Sby. T. Norrisil, Sby.

Trochiscus Norrisii, Sby.; Mag. N. Hist., 2 Scr.

T. convexus, Cpr.; Brit. Assn. Rep., 1863, p. 652 (juv.).

Post-Pliocene, Santa Barbara. Living.

LEPTOTHYRA, Cpr. L. BACUla, Cpr.

Leptonyx bacula, Cpr.; Brit. Assn. Rep., 1863, p. 652.

Leptothyra bacula, Cpr.; Brit. Assn. Rep., 1866.

Post-Pliocene, Santa Barbara. Living.

L. sanguinea, Cpr.

Leptonyx sanguinea, Cpr.; Brit. Assn. Rcp , 1863, p. 652. Leptothyra sanguinca, Cpr.; Brit. Assn. Rep., 1866. Post-Pliocene, Santa Barbara. Living.

MARGARITA, Leach. M. ACUTicostata, Cpr.

Margarita acuticostata, Cpr.; Brit. Assn. Rep., 1863, p. 653.

Post-Pliocene, San Pedro. Living.

M. Salmonea, Cpr.

M. salmonea, Cpr.; Brit. Assn. Rep., 1863, p. 653.

Post-Pliocene, Santa Barbara. Living.

HALIOTIS, Linn. H. RuFescens, Sby.

Maliotis rufescens, Sby.; App. to Bligh. Cat., p. 2.

Id., Reeve ; Icon. Conch., pl. 2, sp. 6.

Post-Plioccne, San Pedro and San Nicholas Island. Living.

IUCAPINA, Gray, L. CRenulata, Sby., sp.

Fissurella crenulata, Sby.; Conch. Mll., No. 19, fig. 31, 38.

Lucapina crenulata, Cpr.; Zool. Proc., 1856, p. 223.

Post-Pliocene, San Pedro. Living.

GLYPHIS, Cpr. G. ASPERA, Esch., sp.

Fissurella aspera, Esch.; Zool. Atl., pt. 5, p. 21, pl. 23, fig. 5.

Glyphis aspera, Cpr.; Zool. Proc., 1856, p. 223.

Post-Pliocenc, San Pedro. Living. 
FISSURELLA, Brug. F. volcano, Rve.

Fissurella volcano, Rve.; Ieon. Conch., pl. 4, sp. 2.

Post-Pliocene, San Pedro. Living.

CLYPIDELla, Swains. C. Bimaculata, Dall.

Clypidella bimaculata, Dall. ; (Mss.) in Coop.; Geog. Cat. W. C. Moll., No. 470.

Post-Plioeene, San Pedro. Living.

\section{C. callomarginata, Cpr.}

C. callomarginata, Cpr.; Brit. Assn. Rep., 1866.

Post-Pliocene, San Pedro. Living.

DENTALIUM, Linn. D. heXagonUM, Sby.

D. hexagonum, Sby.; Thes. Coneh., Vol. 3, p. 103, fig. 10.

Post-Plioecne, San Pedro. Living.

ACRAA, Esch. A. PATina, Esch.

Acmaca patina, Eseh ; Zool. Atl., 1831, p. 19, pl. 24, fig. 7-9.

Post-Pliocene, Santa Barbara. Living.

A. persona, Esch.

A. persona, Esch.; loc. cit., p. 21, pl. 24, fig. 1, 2.

Post-Plioeene, San Nieholas Island. Living.

$$
\text { A. scabra, Nutt., sp. }
$$

Lottia scabra, Nutt.; Jay's Cat., No. 2907.

Patella (Lottia) scabra, Gld.; Bost. N. II. Soc. Proe., 1846, p. 152.

$I d$., Gld.; Wilkes's Exped., p. 348, pl. 32, tig. 456.

Acmoea scabra, Cpr.; l'roc. Zool. Soe., 1856, p. 222.

Post-Plioeene, Santa Barbara. Living.

$$
\text { A. spectrum, Nutt., sp. }
$$

Lottia spectrum, Nutt.; Jay's Cat., No. 2877.

Patella (Lottia) spectrum; Gld. ; W. C. shells, p. 10.

Acmoea spectrum, Cpr.; Brit. Assn. Rep., 1866.

Post-Plioeene, San Pedro, San Nicholas Island. Living. 
A. Rudis, Gabb.

A. rudis, Gabb ; Pal. Cal., Vol. 2, p. 51, p.. 14, fig. 9, 9 a.

Pliocene, San Fernando.

SCURRIA, M. Edw. S. Mitra, Esch., sp.

Acmcea mitra, Esch.; Zool. Atlas, 1833, p. 18, pl. 23, fig. 4.

A. mamillata, Esch.; loc. cit., p. 18.

A. marmorea, Esch.; loc. cit., p. 19.

Patella scurra, Less.; Voyage Coquille, p. 421.

Acmøa scurra, d'Orb.; Am. Mer., p. 478.

Patella (Acmaea) scurra, Midd.; Mal. Ross., Pt. 2, p. 34.

Scurria mitra, Gray; Genera, 1856.

Lottia pallida, Gray ; Zool. Beechey's Voy., p. 147, pl. 39, tig. 1.

Post-Pliocene, San Pedro. Living.

NACELLA, Gray. N. Insessa, Hds., sp.

Patella insessa, Hds.; An. Mag. Nat. Hist., Vol. 10, p. 82.

Nacella insessa, Cpr.; Brit. Assn. Rep., 1863, p. 650.

Post-Pliocene, San Pedro. Living.

\section{CRYPTOCHITON, Gray. C. Stelderir., Midd.}

Chiton Stellerii, Midd.; Bull. Acad. S. Pet., Vol. 6, p. 116.

Chiton (Cryptochiton) Stellerii, Midd.; Mal. Ross., pt. 1, p. 93, pl. 1, fig. 1, 2.

C. amiculatus, Sby.; Conch. Ill., Chitons, fig. 80.

C Sitkensis, Rve.; Icon. Conch., pl. 10, sp. 55.

? C. chlamys, Rve., loc. cit., sp. 60.

C. Californicus, Prescott; Am. Jour. Sci., 1864, p. 185.

Post-Pliocene, San Diego. Living.

BULla, Linn. B. nebulosa, Gld.

Bulla nebulosa, Gld.; in A. Ad.; Thes. Conch., Vol. 2, p. 578, pl. 123, fig. 79,80 .

Pliocene, San Fernando. Living.

B. Adamsi, Mke.

Bulla Adamsi, Mke.; Zeitsch., 1850, p. 162.

Post-Pliocene, San Pedro. Living. 
TORNATINA, A. Ad. T. culcitella, Gld., sp.

Bulla (Akera) culcitella, Gld.; Mex. \& Cal. Shells, p. 14, pl. 14, fig. 8.

Tornatina culcitella, Cpr.; Proe. Zool. Soc., Lond., 1856, p. 227.

Post-Pliocene, Santa Barbara. Living.

Besides the above Marino Univalves, several Helices have been found in a fossil state, usually in isolated deposits of doubtful age. The following are mentioned in my memoranda:

II. Mormonum, Pfr.

Plioeene, with human skull, near Angels' Camp, Calaveras County.

II. tudiculata, Binney.

Post-Pliocene, Marinc deposits of San Pedro.

H. Hillebrandi, Newe.

Ncar Mariposa.

II. Tryoni and II. facta, Newc.; curious varieties from the islands off the southern coast. The former is thiek and, in some eases, subangulated; the latter is much larger than the living forms, and very solid.

\section{ACEPHALA.}

ZIRPH

Z. Gabbii, Tryon; Proc. Phil. Acad. Nat. Sei., 1866, p. 144, pl. 1, fig. 1.

Z. Gabbii, Gabb ; Pal. Cal., Vol. 2, p. 52, pl. 15, fig. 10.

Z. crispata, Cpr., Coop., Auct. (non Linn.).

Post-Pliocenc, San Pedro. Living.

Penitella, Val. P. penita, Con., sp.

Pholas penita, Con.; Jour. A. N. S., 1 Ser., Vol. 7, p. 237, pl. 18, fig. 7.

Penitella Conradi, Val.; Voy. Venus, pl. 24, tig. 1.

Parapholas penita, Cpr.; Zool. Proc., 1856, p. 210.

Pholadidea penita, Cpr. ; Brit. Assn. Rep.

Penitella speloca, Con.; P. R. R. Rep., Vol. 5, p. 326, pl. 5, fig. 43.

Penitella penita, Tryon; Proe. Phil. A. N. S., 1861, Monog. Pholad., p. 87

Post-Pliocene, Santa Barbara. Living.

SOLEN, Linn. S. Rosaceus, Cpr.

Solen ? var. rosaceus, Cpr.; Brit. Assn. Rep., W. C. Moll., 1863, p. 638.

Plioeene, San Fernando, Santa Rosa. Living. 
Sub-Genus HYPogella, Gray. Solena, Browne (Pre-Lin.).

S. (H.) PRoteXta, Con., sp.

Donax protexta, Con.; Wilkes's Exped., p. 728, pl. 17, fig. 9.

Solena protexta, Con. ; Jour. Conch., 1865, p. 152.

Plectosolen protexta, Con.; Smithsonian Check List, No. 242.

Miocene, Astoria.

SILIQUARIA, Schum. ? S. EDENTULA, Gabb.

3 S. edentula, Gabb, Pal. Cal., Vol. 2, p. 53, pl. 15, fig. 11.

Pliocenc, San Fcrnando.

IACH ERA, Gld. M. PATULA, Dixon, sp.

Solen patulus, Dixon; Voyage Around the World, \&c., p. 355, fig. 2.

Machara patula, Cpr.; Rep. W. C. Moll., 1863, p. 638.

Solen maxima, Wood.; Gen. Conch., pl. 31, fig. 3.

Solecurtus Nuttallii, Con.; Jour. Phil. A. N. S., 1 S., Vol. 7, p. 232, pl. 17, fig. 9.

Solemya ventricosa, Con.; Wilkes's Exped., p. 723, pl. 17, fig. 7, 8 .

Id., Con.; Jour. Conch., 1865, p. 153.

Siliqua patula, Con.; Jour. Conch., 1867, app., Cat. Solenidæ, p. 25.

Siliqua Californica, Con.; Jour. Conch., 1867, p. 193.*

Miocene, Astoria, Martinez, Tomales, and Max. Martinez Ranch, Santa Clara County; Pliocenc, Santa Rosa. Living.

SOLECURTUS, Blainv. S. subteres, Con.

S. subteres, Con.; Jour. A. N.S. Phil., 1 Scr., Vol. 7, p. 233, pl. 17, fig. 10. Post-Pliocene, Santa Barbara. Living.

GLYCIMERIS, Lam. G. generosa, Gld., sp.

Panopaea generosa, Gld.; Proc. Bost. N. H. Soc., 1850, p. 215.

Id., Gld.; Wilkes's Exped., Moll., p. 385, pl. 34, fig. 507.

* Described from a young specimen, differing only in being a little straighter than usual on its basal margin. It seems incomprehensible to a Californian conchologist, how Mr. Conrad could have redescribed this familiar form, for which he had already made threcsynonyms, and with the fineseries in the Academy's museum for comparison. "Body Bay," its locality, should read Bodega Bay. "Nuttallii" has been persistently referred by Mr. Conrad to the Sandwich Islands. It does not occur there, but is one of the commonest of our Californian shclls.

PAL. VOL. II. -13 
Glycimeris generosa, H. \& A. Ad.; Gen. Rec. Moll., p. 350.

Mya abrupta, Con.; Wilkes's Exped. Foss., p. 723, pl. 17, fig. 5.

Glycimeris abmupta, Con. ; Jour. Conch., 1865, p. 152.

G. Estrellanus, Con.; P. R. R. Rep., Vol. 7, p. 194, pl. 7, fig. 5.

Miocene, Oregon, Martinez, Walnut Creek, near Mount Diablo, Esirella, Foxin's; Plioeene, San Fernando, Santa Barbara; Post-Pliocene, Santa Barbara. Living.

CRYptomya, Con. C. Califonnica, Con.

C. Californica, Con.; Proc. Phil. Aead., 1849, p. 121.

Sphania Californica, Con.; Jour. A. N. S., 1 Ser., Vol. 7, p. 234, pl. 17, fig. 11. Sphania Californica, Cpr.; Zool. Proe., 1856, p. 210.

Cryptomya ovalis, Con.; P. R. R. Rep., Vol. 6, p. 69, pl. 2, fig. 2.

Miocene, Griswold's; Pliocene, San Fernando, Santa Rosa; Post-Plioeene, Santa Barbara. Living.

MYtilimeria, Con. M. Nuttallit, Con.

Mytilimeria Nuttallii, Con.; Jour. A. N. S., 1 Ser., Vol. 7, p. 247.

Mioeene, Tomales; Post-Plioeene, San Pedro. Living.

THRACIA, Leach. 'T. TrapezoIdes, Con.

T. trapezoides, Con.; Wilkes's Exped., p. 723, pl. 17, fig. 6.

Mioeene, Astoria ; Plioeene, Eagle Prairie.

PANDORA, Brug. P. SCAPHA, Gabb.

Pandora scapha, Gabb ; Pal. Cal., Vol. 2, p. 18, pl. 4, fig. 32.

Miocene, Martinez.

Clidophora, Cpr. C. punctata, Con., sp.

Pandora punctata, Con.; Jour. Phil. Aead., 1 S., Vol. 7, p. 288, pl. 17, fig. 1. Clidophora punctata, Cpr.; Proc. Zool. Soe., 1864, p. 598.

Miocene, near San Buenaventura. Living.

HEMIMACTRA, Swains. H. Lenticularis, Gabb.

H. lenticularis, Gabr ; Pal. Cal., Vol. 2, p. 19, pl. 4, fig. 33.

Miocene, Martinez. 
? H. occidentalis, Gabb.

? Hemimactra occidentalis, Gabb ; Pal. Cal., Vol. 1, p. 54, pl. 15, fig. 18, $13 a$. Miocene, Martinez.

SCHIZODESMA, Gray. S. ABscissa, Gabb.

Schizodesma abscissa, Gabb ; Pal. Cal., Vol. 2, p. 20, pl. 4, tig. 34. ${ }^{\natural}$

Mulinea densata, Con.; pars, P. R. R. Rep., Vol. 6, p. 71.

Miocene, Martinez, San Pablo, Walnut Creek.

mUlinea, Gray. M. Densata, Con.

Mulinea densata, Con. ; pars, P. R. R. Rep., Vol. 6, p. 71, pl. 3, fig. 12.

Mulinea densata, Gabb ; Pal. Cal., Vol. 2, p. 19, pl. 5, fig. 35.

Miocene, Santa Barbara, San Pablo, Martinez, Walnut Creek, hills back of Oakland.

SCHIZOTHERUS, Con. S. Nuttalli, Con.

Lutraria (Cryptodon) Nuttalli, Con.; Jour. A. N. S., Vol. 7, 1 S., p. 235, pl. 18, fig. 1.

Schizothoerus Nuttalli, Con.; P. R. R. Rep., Vol. 5, p. 324, pl. 4, fig. 33.

Lutraria maxima, Midd. ; Mal. Ross., 1849, p. 66, pl. 19, fig. 1, 4.

Mactra maxima, Rve.; Icon. Conch., pl. 1, sp. 4.

Lutraria capax, Gld.; Proc. Bost. N. H. Soc., 1850, p. 217.

Lutraria maxima, Gld.; Wilkes's Exped., p. 395, pl. 34, fig. 508.

Tresus maximus, H. \& A. Ad.; Gen. Rec. Moll.

Pliocene, Santa Barbara; Post-Pliocene, Santa Barbara, San Diego, San Pedro. Living.

STANDELLA, Gray. S. PlanUlata, Con., sp.

Mactra planulata, Con.; Jour. Phil. Acad., 1 Ser., Vol. 7, p. 240.

M. Diegoana, Con.; P. R. R. Rep., Vol. 5, p. 325, pl. 5, fig. 45.

M. albaria, Con. ; Am. Jour. Sci., 1848, p. 432, fig. 4.

Standella planulata, Cpr.; Brit. Assn. Rep., 1863, p. 640.

Miocene, Oregon, Max. Martinez Ranch, Martinez; Pliocene, San Fernando;

Post-Pliocene, San Diego. Living. 
S. FAlCATA, Gld. sp.

Mactra falcata, Gld.; Otia, p. 76.

Standella falcata, Cpr.; Brit. Assn. Rep., 1863, p. 640.

Pliocene, Kirkcr's Pass. Living.

PSEUDOCARDIUM, Gabb. P. Gabii, Rém., sp.

Cardium Gabbii, Rémond ; Proc. Cal. Acad., 1863, p. 13.

Pseudocardium id., Gabb; Pal. Cal., Vol. 2, p. 21, pl. 6.

Mioccne, Martincz and Celro Bonito, Monterey County; Pliocenc, Kirker's Pass.

GaRI, Sehum. G. (Psammocola) alata, Gabb.

G. (Psammocola) alata, Gabb; Pal. Cal., Vol. 2, p. 21, pl. 5, fig. 36. Plioccne, Kirker's Pass.

TELIINA, Linn. T. Congesta, Con.

Tellina congesta, Con.; P. R. R. Rep., Vol. 5, p. 323, pl. 3, fig. 14, 18-21.

Miocenc, Montcrey, San Pablo, San Diego, etc.

T. Bodegensis, Hds.

Tellina Bodegensis, Hds.; Voy. Sulphur, p. 67, pl. 21, fig. 2.

T. emacerata, Con.; Wilkes's Exped., p. 725, pl. 18, fig. 4.

Id., Con.; Sm. Check List, No. 206; and Jour. Conch, 1865, p. 152.

Miocene, Astoria; Post-Pliocene, San Pedro. Living.

LUTRICOLA, Blainv. I. Alta, Con., sp.

Tellina alta, Con.; Jour. Phil. Acad., Vol. 7, p. 258.

Lutricola alta, Cpr.; Brit. Assn. Rep., p. 639.

Arcopagia medialis, Con.; P. R. R. Rep., Vol. 7, p. 72, pl. 2 , fig. 6 .

Miocene, Monterey ; Plioccne, San Fernando, Santa Barbara ; .Post-Pliocene, San Pedro. Living.

L. viridotincta, Cpr.

Lutricola viridotincta, Cpr.; Brit. Assn. Rep., 1866.

Scrobicularia viridotincta, Cpr.; Proc. Zool. Soc., 1856, p. 160.

Post-Pliocene, Santa Barbara. Living. 
ANGULUS, Muhlf. A. Gouldi, Hanley.

Tellina Gouldii, Hanley; Thes. Conch., Vol. 1, p. 272, pl. 56, fig. 26.

Angulus Gouldii, Hanley; Ms. (Cpr.), Brit. Assn. Rep., 1863, p. 639.

Post-Pliocene, San Diego. Living.

MACOMA, Leach. M. SECTA, Con., sp.

Tellina secta, Con.; Jour. Phil. Acad., 1 scr., Vol. 7, p. 257.

Macoma secta, II. \& A. Ad.; Genera, p. 401.

Tellina ligamentina, Desh.; Guer. Mag., 1843, pl. 8.

Post-Pliocene, Santa Barbara. Living.

\section{EDuris, Nutt. sp.}

Macoma var. edulis, Nutt.; Cpr.; Brit. Assn. Rep., 1863, p. 639.

Pliocene, San Fernando, Santa Barbara ; Post-Plioeene, San Pedro. Living.

\section{YoLdiformis, Cpr.}

Macoma yoldiformis, Brit. A. Rep., 1863, p. 639.

Post-Pliocene, Santa Barbara. Living.

\section{M. nasuta, Con., sp.}

Tellina nasuta, Con.; Jour. Phil. Acad., Vol. 7, p. 258.

? T. Oregonensis, Con.; Am. Jour. Sci., 1848, p. 432, fig. 5.

T. tersa, Gld.; Mex. and Cal. Shells, p. 25, pl. 16, fig. 2.

Macoma nasuta, Cpr.; Brit. Assn. Rep., 1863, p. 639.

Upper Miocenc, Foxin's; Pliocene, San Fernando, Santa Rosa; Post-Pliocene, San Pedro. Living.

M. Inquinata, Desh., sp.

Tellina inquinata, Desh.; Proc. Zool. Soc., 1854, p. 357.

Macoma inquinata, Cpr.; Rep. W. C. Moll., Brit. Assn., 1863, p. 639.

Post-Pliocene, San Dicgo. Living.

\section{M. expansa, Cpr.}

M. expansa, Cpr. ; Brit. Assn. Rep., 1863, p. 639.

Post-Pliocene, San Pedro. Living. 


\section{Pebroana, Con., sp.}

Tellina Pedroana, Con.; P. R. R. Rep., Vol. 5, p. 323, pl. 3, fig. 17.

Post-Pliocene, San Pedro. Living.

M. Inconspicua, Brod. \& Sby., sp.

Tellina inconspicua, B. \& S.; Zool. Jour., 1829, p. 363; Beech ey's Voy., p. 153, pl. 41 , fig. 6 .

Sanguinolaria Californica, Con.; Jour. Phil. Acad., Vol. 7, p. 231, pl. 17, fig. 11. Macoma inconspicua, Cpr.; Brit. Assn. Rep., 1863.

Post-Pliocene, Santa Barbara. Living.

\section{SEMELE, Schum. S. DECISA, Con., sp.}

Amphidesma decisa, Con.; Jour. Phil. Acad., 1 ser., Vol. 7, p. 234, pl. 17, fig. 12. Semcle decisa, Cpr. ; Proc. Zool. Soc., 1856, p. 213.

Post-Pliocene, San Pcdro. Living.

$$
\text { S. pulchra, Sby., sp. }
$$

Amphidesma pulchra, Sby.; Conch. Ill., No. 2, fig. 2.

Semele pulchra, Cpr. ; Rep. W. C. Moll., Brit. Assn., 1856, 1863.

Post-Pliocene, San Pedro. Living.

CUmingia, Sby. C. Californica, Con.

Cumingia Californica, Con.; Jour. Phil. Acad., 1 ser., Vol. 7, p. 234, pl. 17, fig. 12.

Post-Pliocenc, Santa Barbara. Living.

MERCENARIA, Schum. M. Perlaminosa, Con.

M. perlaminosa, Con.; Proc. Phil. Acad., 1855, p. 267.

Id., Gabb; Pal. Cal., Vol. 2, p. 22, pl. 5, fig. 38; p. 55, pl. 15, fig. 14.

Venus Kennerleyi, Rve.; Icon. Conch., Venus, pl. 12, fig. 41.

Mercenaria Kennerleyi, Cpr.; Rep. on W. C. Moll., 1866.

Post-Pliocene, Santa Barbara. Living.

Chione, Megerle. C. succincta, Val., sp.

Venus succincta, Val.; Humb. \& Bonpl., Obs. sur. Zool., p. 219, pl. 48, fig. 1. Chione succincta, Cpr.; Rep. W. C. Moll., Brit. Assn., 1863, p. 641.

Venas Californica, Con.; Jour. Phil. Acad., Vol. 7, p. 251. 
V. Nuttallii, Con. loc. cit., p. 250, pl. 19, fig. 14 .

V. lamellifera, Con.; Wilkes's Exped., Geology, p. 724, pl. 17, fig. 12.

V. lamellifera, Con.; Jour. Conch., 1865, p. 152.

Not V. lamellifera, Con. Jour. Phil. Acad., Vol. 7, p. 251, pl. 19, fig. 19, (=

Tapes straminea var. ruderata.)

V. brevilineata, Con.; Wilkes's Exped., p. 724, pl. 17, fig. 13.

Dione brevilineata, Con.; Jour. Conch., 1865, p. 152.

Venus securis, Shum.; Trans. St. Louis Acad., Vol. 1, p. 122.

Miocene, Martinez, Griswold's, Foxin's, San Pablo; Oregon, Coose Bay, Astoria, Eugene City ; Pliocene, San Fernando, Santa Rosa ; Post-Pliocene, San Pedro, Santa Barbara, San Diego. Living.

\section{Mathewsonit, Gabb.}

C. Mathewsonii, Gabb; Pal. Cal., Vol. 2, p. 23, pl. 5, fig. 39.

Miocene, Martinez.

\section{Whitneyi, Gabb.}

C. Whitneyi, Gabb ; Pal. Cal., Vol. 2, p. 23, pl. 5, fig. 40.

Miocene, Martinez.

\section{Pertenuis, Gabb.}

Venus Kennerleyi ? Gabb, not Reeve ; Pal. Cal., Vol. 2, p. 22, pl. 5, fig. 37.

$V$. pertenuis, Gabb; loc. cit., note.

Miocene, Martinez.

\section{Oregona, Con., sp.}

Cytherea Oregona, Con.; Am. Jour. Sci., 1848, p. 432, fig. 8.

Dione Oregona, Con.; Jour. Conch., 1865, p. 152.

Miocene, Astoria.

\section{CARYATIS, Roem. C. Barbarensis, Gabb.}

C. Barbarensis, Gabb ; Pal. Cal., Vol. 2, p. 56, pl. 15, fig. 15, 15 a. Pliocene, Santa Barbara.

\section{HERETRIX, Lam. M. Traskit, Con. sp.}

Lutraria Traskir, Con.; P. R. R. Rep., Vol. 5, p. 324, pl. 3, fig. 23.

Meretrix Traskii, Gabb; Pal. Cal., Vol. 2, p. 56.

Miocene, Monterey County. 
PSEPHIS, Cpr. P. Tantilla, Gld., sp.

Venus tantillus, Gld.; Shclls of Cal. and Mex., p. 33, pl. 15, fig. 10.

Trigona tantilla, Cpr.; Proc. Zool. Soc., 1856, p. 201.

Venus rhysomia, Gabb; Proc. Phil. Acad., 1861, p. 371.

Psephis tantilla, Cpr.; Rep. W. C. Moll., 1863, p. 640.

Post-Pliocene, Santa Barbara. Living.

\section{P. Lord, Baird, sp.}

Chione Lordi, Baird ; Proc. Zool. Soc., 1863, p. 69.

Psephis Lordi, Cpr.; Rep. W. C. Moll., 1863, p. 641.

Post-Pliocene, Santa Barbara. Living.

PACHYDESMa, Con. P. crassatelloides, Con.

Cytherea (Trigonella) crassatclloides, Con.; Jour. Phil. Acad., Vol. 7, p. 253, pl. 19 , fig. 17.

Trigona crassatelloides, Desh. ; Cat. Ven., p. 46.

Pachydesma crassatelloides, Con.; Proc. Phil. Acad., 1854, p. 121.

Donax stultorum, Mawe; Coneh., pl. 9, fig. 7.

Trigona stultorum, Gray, Analyst; and Ianley; Sp. Shclls, p. 105.

Cytherea solidissima, Phil.; Zcits. fur. Mal., 1851, p. 74, No. 100.

Post-Pliocene, San Diego. Living.

Callista, Poli. C. (? Tapes) Voyi, Gabb.

Callista Voyi, Gabb; Pal. Cal., Vol. 2, p. 24, pl. 5, fig. 41.

Pliocene, Eagle Prairic, Humboldt County.

LIOCONCHA, Mörch. L. Newcombiana, Gabb.

Linconcha Newcombiana, Gabb; Proc. Cal. Acad., 1865.

Post-Pliocene, San Diego. Living.

amiantis, Cpr. A. callosa, Con., sp.

Cytherea callosa, Con.; Jour. Acad., Vol. 7, p. 252.

Venus callosa, Sby.; Thes. Conch., Vol. 2, p. 712, pl. 154, fig. 44, 45.

Dosinia callosa, Cpr.; Zool. Proc., 1856, p. 216.

Amiantis callosa, Cpr.; Rep. W. C. Moll., Brit. Assn., p. 640.

Post-Pliocenc, Santa Barbara. Living. 
DosinIa, Scopoli. D. Conradi, Gabb.

D. Conradi, Gabb; Pal. Cal., Vol. 2, p. 25, pl. 5, fig. 43.

D. alta, Con.; (not Dkr.), Proc. Phil. Acad.; 1856, p. 315.

Not D. alta, Con.; P. R. R. Rep., Vol. 5, p. 320, pl. 2, fig. 2, (= D. elevata G.)

D. alta, Con.; P. R. R. Rep., Vol. 6, p. 71, pl. 3, fig. 13.

D. alta, Con.; P. R. R. Rep., Vol. 7, p. 193.

Miocene, San Emidio, Montercy.

\section{D. ponderosa, Gray, sp.}

Artemis ponderosa, Gray; Analyst, 1838, Vol. 8, p. 309.

Cylherea gigantea, Sby.; Phil. Abbild., pl. 7, fig. 1.

Dosinia ponderosa, H. \& A. Ad.; Gen. Rec. Moll., Vol. 2, p. 432.

Post-Pliocene, Kirker's Pass, Santa Barbara. Living.

D. Mathewsonit, Gabb.

Dosinia Mathewsonii, Gabb ; Pal. Cal., Vol. 2, p. 57, pl. 15, fig. 16.

Miocene, Martinez, Griswold's.

TAPES, Megerle. T. Staminea, Con. sp.

Venus staminea, Con.; Jour. Phil. Acad., Vol. 7, p. 250, pl. 19, fig. 15.

Venus lamellifera, Con.; loc. cit., p. 251, pl. 19, fig. 19, (= var. ruderata.)

Not V. lamellifera, Con.; Wilkes's Exped., p. 724, pl. 17, fig. 12, (= Chione succincta.)

Tapes diversum, Con.; P. R. R. Rep., Vol. 5, p. 324, pl. 4, fig. 31.

T. lineatum, Con. ; P. R. R. Rep., Vol. 6, p. 72, pl. 2, fig. 7.

Venus rigida, Gld.; Proc. Bost. N. Hist. Soc., 1850, p. 277.

Id., Gld ; Wilkcs's Exped., p. 420, pl. 37, fig. 538.

Tapes staminea, Cpr.; Brit. Assn. Rep., W. C. Moll., 1863, p. 641.

Miocenc, Santa Clara County, Foxin's; Pliocene, Kirker's Pass, San Fernando, Santa Rosa; Post-Pliocene, Santa Barbara, San Pedro. Living.

T. tenerrima, Cpr.

Tapes tenerrima, Cpr.; Zool. Proc. 1856, p. 200.

Pliocene, Santa Barbara. Living.

PAL. VOL. II. -14 


\section{T. Staleyi, Gabb.}

Dosinia Staleyi, Gabb; Pal. Cal., Vol. 2, p. 24, pl. 7, fig. 42.

Tapes id., Gabb; Index to platr, loc. cit.

Tapes id., Gabb; Pal. Cal., Vol. 2, p. 57, pl. 16, fig. 17, 17 a.

Plioeene, Santa Rosa.

\section{?'T. TRUncata, Gabb.}

Tapes? truncata, Gabb; Pal. Cal., Vol. 2, p. 25, pl. 7, fig. 44.

Mioeene, Griswold's.

SAXIDOMUS, Con. S. gracilis, Gld., sp.

Tapes gracilis, Gld.; P. R. R. Rep., Vol. 5, p. 333, pl. 19, fig. 20.

Suxidomus aratus, Gld.; 1861, Otia, p. 168.

Venus maxima, Phil.; Abbild., Vol. 2, pl. 6, fig. 1.

Saxidomus gracilis, Cpr. ; Brit. Assn. Rep., 1866.

Mioeene, Santa Cruz, Walnut Creek, Martinez, Santa Inez; Plioeene, San

Fernando, Santa Barbara; Post-Plioeene, Santa Barbara. Living.

\section{S. Nuttalli, Con.}

Saxidomus Nuttalli, Con.; Jour. Phil. Aead., Vol. 7, p. 249, pl. 19, fig. 12. Post-Plioeene, Santa Barbara, San Pedro. 'Living.

\section{S. gibbosus, Gabb.}

Saxidomus gibbosus, Gabb; Pal. Cal., Vol. 2, p. 58, pl. 16, fig. 18, a, b. Plioeene, Eagle Prairie, Humboldt County.

cyrena, Lam. C. Californica, Gabb.

Cyrena Californica, Gabb; Pal. Cal., Vol. 2, p. 26, pl. 7, fig. 45.

Plioeene, Kirker's Pass.

CARDIUM, Linn. C. CoRBIs, Martyn, sp.

Pectunculus corbis, Mart.; Univ. Coneh., pl. 28, fig. 2.

C. corbis, (Desh. Mss.) Cpr.; Brit. Assn. Rep., 1863, pp. 527, 642.

C. Californianum, Con.; Jour. A ead. 1'hil., Vol. 7, p. 229, pl. 17, fig. 4.

C. Nuttalli, Con.; loc. cit., p. 229, pl. 17, fig. 3.

Plioeene, Twelve-mile House, below San Franeiseo, San Fernando; Post-

Pliocene, Santa Barbara, San Pedro, San Franeiseo. Living. 


\section{Meekianum, Gabb.}

C. Meckianum, Gabb ; Pal. Cal., Vol. 2, p. 27, pl. 7, fig. 46. Plioeene, Eagle Prairie.

\section{C. centifilosum, Cpr.}

Cardium centifilosum, Cpr.; Rep. W. C. Moll., 1863, p. 642. Post-Plioeene, San Pedro. Living.

\section{Panamense, Sby.}

Cardium Panamense, Sby.; Proe. Zool. Soe, 1833, p. 85.

Post-Plioeene, San Pedro. Living.

\section{Sub-Genus LEVICARDIUM, Swains. C. (L.) ELATUM, Sby.}

Cardium elatum, Sby., Rve.; Icon. Coneh., pl. 8, fig. 41.

Id., Val.; Voy. Venus, pl. 17, fig. 1.

Liocardium elatum, Cpr. ; B. Assn. Rep., 1863, p. 642.

Post-Plioeene, San Diego. Living.

\section{C. (L.) substriatum, Con.}

Cardium substriatum, Con.; Jour. Phil. Aead., Vol. 7, p. 228, pl. 17, fig. 2. Liocardium substriatum, Cpr.; Br. Assn. Rep., W. C. Moll., 1863, p. 642.

Post-Plioeene, San Pedro. Living.

\section{CONCHOCELE, Gabb. C. DISJuncta, Gabb.}

Conchocele disjuncta, Gabb ; Pal. Cal., Vol. 2, p. 28, pl. 7, fig. 48.

Post-Plioeene, Dead Man's Island, off San Pedro.

This is a small detached mass of very recent Tcrtiary, rich in fossils, all of living species, except the present one. I quoted it as "probably mioeene," in deseribing the speeies, but have sinee obtained more definite information by a personal visit to the loeality.

\section{Bisecta, Con., sp.}

Venus bisecta, Con.; Wilkes's Exped., p. 724, pl. 17, fig. 10.

Cyprina bisecta, Con.; Jour. Coneh., 1865, p. 153.

Id., Con.; Cheek List, No. 160.

Mioeene, Astoria. 
IUCINA, Brug.' L. BoREALIS, Linn., sp.

L. borealis, Linn.; Syst. Nat., Ed. 12, p. 1413.

L. acutilineata, Con.; Wilkes's Exped., p. 725, pl. 18, fig. 2.

Cyclas acutilineatus, Con.; Jour. Coneh., 1865, p. 153.

Pectunculus patulus, Con.; Jour. Conch., 1865, p. 153.

Not P. patulus, Con.; Wilkes's Exped., p. 726, pl. 18, fig. 8, (= Axincea id.)

Lucina tetrica, Con. ; Proe. Phil. Acad., 1856, p. 314.

Mioeene, Oregon, Foxin's, Martinez, Griswold's, Orestimba Cañon; Pliocene, San Fernando, Santa Rosa; Post-Plioeene, Santa Barbara, San Pedro, Dead Man's Island, San Diego, ete. Living.

\section{Nuttalli, Con.}

L. Nuttalli, Con.; Jour. Phil. Acad., Vol. 7, p. 255, pl. 20, fig. 2. Post-Pliocene, San Pedro, Santa Barbara. Living.

\section{Californica, Con.}

L. Californica, Con.; Jour. Phil. Aead, Vol. 7, p. 255, pl. 20, fig. 1. Post-Plioeene, San Pedro, Santa Barbara, Living.

Sub-Genus HeRE, Gabb. L. (H.) Richthofeni, Gabb.

L. (II.) Richthofcni, Gabb; Pal. Cal., Vol. 2, p. 29, pl. 8, fig. 49 Pliocene, San Fernando.

IFYSIA, Leach. II. PARILIS, Con.

Loripes parilis, Con.; Am. Jour. Sei., 1848, p. 433, fig. 7.

Mysia parilis, Con.; Jour. Coneh., 1865, 1. 153.

Mlioeene, Oregon.

Crassatella, Lam. C. collina, Con.

Crassatella cullina, Con.; P. R. R. Rep., Vol. 7, p. 193, pl. 6, fig. 1, 2. Id., Gabb; Pal. Cal., Vol. 2, p. 29, pl. 8, fig. 50.

Miveene, Santa Inez MIts., Ojai Raneh, Santa Barbara County.

\section{CARDita, Lam. C. ventricosa, Gld.}

Cardita ventricosa, Gld.; Proe. Bost. N. H. Soe., 1850, p. 276.

Id., Gld.; Wilkes's Exped., p. 417, pl. 36, fig. 532. 
C. subtenta, Con.; Wilkes's Exped., p. 726, pl. 18, fig. 12.

Cardium subtentum, Con. ; Jour. Concl., 1865, p. 153.

Cardita monilicosta, Gabb; Proc. Phil. Acad., 1861, p. 371.

Cardita occidentalis, Con.; Proc. Phil. Acad., 1855, p. 267.

Cardita occidentalis, Con.; P. R. Tr. Rep., Vol. 6, p. 73, pl. 5, fig. 24.

Venericardia borcalis var. ventricasa, Cpr.; B. A. Rep., W. C. Moll., p. 642.

Miocene, Astoria, Santa Monica, Foxin's; Pliocene, Santa Barbara, San

Fernando ; Post-Plioccne, Santa Barbara, San Pedro. Living.

\section{IrYTiLUS, Linn. M. Californianus, Con.}

Nytilus Californianus, Con.; Jour. Phil. Acad, Vol. 7, p. 242.

Pliocene, San Fernando; Pust-Plioccne, Santa Barbara. Living.

\section{MathewsoniI, Gabb.}

M. Mathewsonii, Gabb; Pal. Cal., Vol. 2, p. 30, c․ 8, fig. 51.

Miocene, Martinez, Santa Monica, San Luis Obispo.

\section{MODIOLA, Lam. M. CAPax, Con.}

Modiola capax, Con.; Jour. Phil. Acad., 1 Ser., Vol. 7, p. 262.

Post-Plioccne, Santa Barbara. Living.

M. RECTA, Con.

Modiola recta, Con.; Jour. Phil. Acad., 1 Scr., Vol. 7, p. 245, pl. 19, fig. 1.

Miocene, El Toro Ranch; Pliocene, Santa Rosa, San Fernando. Living.

\section{Ftabeldata, Gld.}

Modiola flabellata, Gld.; Otia, p. 93.

Pliocene, San Fernando. Living.

M. hultiradiata, Gabb.

Mocliola multiradiata, Gabb ; Pal. Cal., Vol. 2, p. 30, pl. 8, fig. 52.

Miocene, Martinez, San Emidio, Walnut Creek.

SEPTIFER, Recluz. S. BIFURCATUs, Rve., sp.

Mytilus bifurcatus, Rve.; Icon. Conch., pl. 9, fig. 41.

Septifer bifurcatus, Cpr.; Brit. Assn. Rep., 1866.

Post-Pliocene, San Diego. Living. 
ARCA, Linn. A. sulcicosta, Gabb.

Arca sulcicosta, Gahh; Pal. Cal., Vol. 2, p. 31, pl. 9, fig. 53.

Pliocene, Santa Rosa.

\section{A. merodonta, Con.}

Arca microdonta, Con.; P. R. R. Rep., Vol. 5, p. 323, pl. 3, fig. 29.

A. canalis, Con.; P. R. R. Rep., Vol. 6, p. 72, pl. 2, fig. 8.

A. trilineata, Con.; P. R. R. Rep., Vol. 6, p. 72, pl. 2, fig. 9.

? A. dcvincta, Con.; q. v. Should these prove to he the same, devincta being the earliest name given, will have to stand as the name of the species.

Mioeene, Martinez, Griswold's, Santa Barbara, near Buena Vista Lake, El Toro and Najohui Ranehes (Uregon ?-devincta); Pliocene, San Fernando.

AXINEA, Poli. A. patula, Con., sp.

Pcctunculus patulus, Con.; Wilkes's Exped., p. 726, pl. 18, fig. 8.

Not P. patulus, Con.; Jour. Conch, 1865, p. 153 (= Lucina borealis).

Axinca Barbarcnsis, Con.; P. R. R. Rep., Vol. 6, p. 73, pl. 3, fig. 11.

Id., Con. ; P. R. R. Rep., Vol. 7, p. 194, pl. 6, fig. 3.

Mioeene, Astoria, San Pablo, Santa Barbara, Martinez; Plioeene, Santa Rosa.

NUCUla, Lam. S. Gen. Acila, H. \& A. Ad. N. (A.) Castrensis, Hinds, sp.**

Nucula Castrcnsis, Hinds; Proc. Zool. Soc., 1843, p. 98.

Id., Hds.; Voy. Sulphur, p. 61, pl. 17, fig. 5.

N. (Acila) Castrensis, H. \& A. Ad., Genera. Ree. Moll., Vol. 2, p. 545.

Nucula diraricata, Con.; Wilkes's Exped., p. 725, pl. 18, fig. 6.

Nucula dicaricata, Con. Am. Jour. Sci., 1848, p. 432, fig. 1.

Nucula decisa, Con.; P. R. R. Rep., Vol. 5, p. 322, pl. 3, fig. 19.

N. Conradi, Meek; Smithsonian Cheek List.

Mioeene, Astoria, Martinez; Plioeene, San Fernando; Post-Pliocene, Sarta

Barbara, San Pedro, San Diego. Living.

* It has been elaimed that this is the same as an English erag speeies, $N$. Cobbollice. I hardly think it neessary to attempt a serious refutation of the idea. It would he eontrary to all reeeived notions of geographieal distribution, to find here an isolated species from an entirely distinet fauna, and out of another ocean. The mistake has arisen from the elose resemblanee that exists between all the species of this sub-genus; a similar eonfusion having been ereated by Mr. Conrad between the present shell, and an allied form from the eretaceous beds of California. 
LEDA, Schum. L. CALATA, IIds., sp.

Nucula calata, Hds. ; Proc. Zool. Soc., 1843, p. 99 ; Voy. Sulph., p. 64, pl. 18, fig. 13.

Leda calata, Hanley; Thes. Conch., Vol. 3, No. 42, fig. 95, 96.

Pliocene, San Fernando; Post-Pliocene, Santa Barbara, San Pedro. Living.

L. cuneata, Sby.

Leda cuntata, Sby.; Thes. Conch., Vol. 3, p. 128, figs. 92, 93.

Post-Pliocene, Santa Barbara, San Pedro. Living.

YOLDIA, Möll. Y. Nasuta, Gabb.

Voldia nasuta, Gabb; Pal. Cal., Vol. 1, p. 216, pl. 32, fig. 287.

Tertiary (horizon?) "Los Angeles."

Y. IMPressa, Con., sp.

Nucula impressa, Con.; Wilkes's Exped., p. 726, pl. 18, fig. 7.

Yoldia impressa, Meek; Sm. Check List, Miocene.

Y. Cooperii, Gabb ; Proc. Cal. Acad., 1865, p. 189.

Y. Cooperii, Gabb; Pal. Cal, Vol. 2, p. 31, pl. 9, fig. 54.

Y. Cooperii, Cpr., Cooper; Enumerations West Coast Mollusca.

Miocene, between Martincz and Monte Diablo, Astoria; Plioccnc, San Fernando; Post-Pliocene, San Pedro. Living.

PeCten, Linn. P. Cerrosensis, Gabb.

P. Cerrosensis, Gabb; Pal. Cal., Vol. 2, p. 32, pl. 9, fig. 55.

Post-Pliocene, Cerros Island, off Lower California.

P. propatulus, Con.

P. propatulus, Con.; Wilkes's Exped., p. 726, pl. 18, fig. 13.

Mioccne, Oregon; Griswold's, Tomales Bay.

P. catilliformis, Con.

P. catilliformis, Con. ; P. R. R. Rep., Vol. 5, p. 329, pl. 9, fig. 83.

Miocene, Ocoya Creek.

P. Pabloensis, Con.

P. Pabloensis, Con.; P. R. R. Rep., Vol. 6, p. 71, pl. 3, fig. 14.

Miocene, San Pablo, Martincz, Max Martinez Ranch. 


\section{P. Peckinam, Gabb.}

P. Peckhami, Gabb; Pal. Cal., Vol. 2, p. 59, pl. 16, fig. 19.

Miocene, San Pablo, Monterey County, Ojai Ranch.

\section{P. Pedroanus, Trask, sp.}

Plagiostoma Pedroana, Trask ; Proc. Cal. Acad., Vol. 1, p. 86, pl. 3, fig. 1. Plag. annulata, Trask; loc. cit., p. 86, pl. 3, fig. 2. Plag. truncata, Trask; loc. cit., p. 86, pl. 3, fig. 3. Pecten Pedrorinus, Gabb; Pal. Cal., Vol. 2, p. 60. Miocene, San l'edro.

\section{P. Hastatus, Sby.}

P. hastatus, Sby.; Thes. Conch., 1847, p. 72, pl. 22, fig. 236.

P. hericeus, Gld.; Proc. Bost. N. Hist. Soc., 1850, p. 236.

Id., Gld.; Wilkes's Exped., p. 457, pl. 42, fig. 570.

P. altiplicatus, Con.; P. R. R. Rep., Vol. 7, p. 191, pl. 3, fig. 2.

Post-Plioeene, Santa Barbara. Living.

\section{P. Islandicus, Müll.}

P. Icelandicus, Müll.; Zool. Dan., p. 248.

P. Islandicus, Chemn.; Coneh. Cab., Vol. 7, p. 65, fig. 615, 616.

P. Fabricii, Phil.; Abbild., p. 3, (101) tab. 1, fig. 5.

P. Pealii, Con. ; Amer. Marine Conch., p. 12, pl. 2, flg. 3.

Post-Pliocene, Santa Barbara. Living.

\section{P. ventricosus, Sby.}

Pecten ventrieosus, Sby. ; Thes. Conch, p. 51, pl. 12, fig. 18, 19, and 26. P. tumidus, Sby.; Proc. Zool. Soc., 1835, p. 109.

Post-Pliocene, San Pedro. Living.

JAHIRA, Schum. J. Dentata, Sby., sp.

Pecten dentatus, Sby.; Thes. Conch., Vol. 1, p. 49, pl. 15, fig. 105, 106 Janira dentata, Cpr.; Brit. Assn. Rep., W. C. Moll., 1863, p. 645.

Vola dentata, H. \& A. Ad.; Gen. R. Moll.

Pecten excrvatus, Val.; Voy. Venus, pl. 19, fig. 1.

Post-Pliocene, Santa Barbara. Living. 


\section{J. BELLA, Con.}

Janira bella, Con. ; P. R. R. Rep., Vol. 6, p. 71, pl. 3, fig. 16.

Pliocene, Santa Barbara.

\section{Liropecten, Con. L. Estrellanus, Con.}

Pallium Estrellanum, Con.; P. R. R. Rep., Vol. 6, p. 71, pl. 3, fig. 15.

Liropecten Estrellanus, Con. ; Proc. Phil. Acad., 1862, p. 291.

Miocene, Estrella.

\section{CRASSICARDO, Con.}

Pallium crassicardo, Con.; Proc. Phil. Acad., 1856, p. 313.

Liropecten crassicardo, Con. ; Proc. Phil. Acad., 1862, p. 291.

Spondylus Estrellanus, Con.; P. R. R. Rep., Vol. 7, p. 191, pl. 1, fig. 3.

Miocene, Estrella; Pliocene, Kirker's Pass, Santa Barbara, San Buenarentura, Ojai Ranch.

\section{VOLAFORMIS, Con.}

Pallium Estrellanum, Con.; P. R. R. Rep., Vol. 7, p. 191, pl. 3, fig. 3, 4. Not P. Estrellanum, Con.; P. R. R. Rep., Vol. 6, p. 71, pl. 3, fig. 15.

Liropecten voloformis, Con.; Proc. Phil. Acad., 1862, p. 291.

Miocene, Estrella, and Valley of Nacismiento River.

\section{Veatchir, Gabb.}

Pecten Veatchii, Gabb; Pal. Cal., Vol. 2, p. 32, pl. 10, fig. 56.

Pliocene, Ojai Ranch; Post-Pliocene, Cerros Island.

\section{HINNITES, Defr. H. GIganteus, Gray.}

Hinnites giganteus, Gray; A. Phil., 1826, p. 103.

Hinnita Poulsoni, Con.; Jour. Phil. Acad., 1834, p. 152, pl. 14.

Hinnites crassa, Con. ; P. R. R. Rep., Vol. 7, p. 190, pl. 2, fig. 1, 2.

Post-Pliocene, Santa Barbara. Living.

\section{anomia, Linn. A. subcostata, Con.}

Anomia subcostata, Con.; Proc. Phil. Acad., 1855, p. 267.

Id., Con. ; P. R. R. Rep., Vol. 5, p. 325, pl. 5, fig. 34.

Pliocene, Colorado Desert.

PAL. VOL. II. - 15 


\section{A. LAMPE, Gray.}

Anomia lampe, Gray; Proc. Zool. Soc., 1849, p. 115.

Post-Pliocene, San Pedro. Living.

OSTREA, O. Bourgeoisir, Rem.

Ostrea Bourgeoisii, Rém. ; Proc. Cal. Acad., 1863, p. 13.

Ill., Gabb; Pal. Cal., Vol. 2, p. 33, pl. 11, fig. 57.

Pliocene, Kirker's Pass.

\section{O. Aтtwoodi, Gabb.}

Ostrea Attwoodii, Gabb; Pal. Cal., Vol. 2, p. 33, pl. 10, fig. 58 ; pl. 11, fig. $58 b$. Miocene or Pliocene, (?) San Lorenzo Valley, Montercy County.

\section{O. Tayloriana, Gabb.}

Ostrea Tayloriana, Gabb; Pal. Cal., Vol. 2, p. 34, pl. 12, fig. 60. Miocene, Santa Inez Mts., near Santa Barbara.

\section{O. Veatchi, Gabb.}

Ostrea Veatchii, Gabb; Pal. Cal., Vol. 2, p. 34, pl. 11, fig. 59.

Post-Pliocene, Cerros Island, and near Lorcto, Lower California.

\section{O. Cerrosensis, Gabb.}

Ostrea Cerrosensis, Gabb; Pal. Cal., Vol. 2, p. 35, pl. 11, fig. 61.

Compare O. gallus, Val.; Voy. Venus.

Post-Pliocene, Cerros Island.

I have received from Prof. Raimondi, of Lima, Peru, an oystcr from the late Tertiary deposits of Payta, which leads me to suspect the specific identity of $\mathrm{Cer}$ rosensis with the form figured by Valenciennes in the Voyage of the Venus, under the name of 0 .gallus. The only important difference is in size. The Peruvian fossil agrees in size with the figure of the recent form ; while my fossil is not more than half as large. Should no other characters show themselves, gallus will have to stand as the name of the species.

$$
\text { O. lurida, Cpr. }
$$

O. lurida, Cpr.; Rep. on W est Coast Mollusca, 1863, p. 645.

Post-Pliocene, Benicia. Living. 
O. vespertina, Con.

Ostrea vespertina, Con.; Jour. Phil. Acad., 2 Ser., Vol. 2, p. 300.

O. vespertina, Con.; P. R. R. Rep., Vol. 5, p. 325, pl. 5, fig. $36,38$.

Pliocene, Colorado Descrt, Santa Barbara.

\section{O. Heermanni, Con.}

Ostrea Heermanni, Con.; Proc. Phil. Acad., 1855, p. 267.

O. Heermanni, Con.; P. R. R. Rep., Vol. 5, p. 326.

Pliocene, Colorado Desert.

\section{O. Titan, Con.}

Ostrea Titan, Con. ; P. R. R. Rep., Vol. 6, p. 72, pl. 4, fig. 17 ; pl. 5, fig. 17 a. Miocene, Kirker's Pass, South of Mount Diablo, Walnut Creek, Estrella, San Luis Obispo, Ojai Ranch, Cuyama R. Cañon, Tulare Valley, and throughout the Coast Range wherever the Upper Miocene is found.

TAMIOSOMA, Con. T. GRegaria, Con.

Tamiosoma gregaria, Con.; Proc. Phil. Acad., 1856, p. 315.

Tamiosoma gregaria, Con.; P. R. R. Rep., Vol. 6, p. 72, pl. 4, fig. 18.

Balanus Estrellanus, Con.; P. R. R. Rep., Vol. 7, p. 195, pl. 8, fig. 1.

Radiolites gregaria, Con.; Proc. Phil. Acad., 1864, p. 214.

Tamiosoma gregaria, Gabb; Pal. Cal., Vol. 2, p. 61, pl. 18, fig. 22, a, b, c, $d$ Miocene, Estrella, Santa Marguerita Ranch, Tulare Valley.

\section{POLYZOA.}

CELLEP0RA, Fabr., Auct. C. Californiensis, G. \&. H.

Cellepora Californiensis, Gabb \& Horn.; Jour. Phil. Acad., 1862, p. 130, fig. 12. Post-Pliocene, Santa Barbara. Living.*

* All the Polyzoa in this list are from the Post-Pliocene murl of Santa Barbara. Most of them, if not all, have bcen found living on the coast, and I have made extensive collections of the recent Pacific forms, which, to my great regret, I have not becn ablc to study, partly for want of time, and partly bccause my eyes will not permit the use of the microscope. My friend, Dr. S. B. Howell, Secretary of the Philadelphia Academy of Natural Sciences, has kindly undertaken the work, and I trust that his results will be as useful as the subject is interesting. I have retained here the Orbignyan generic names and classification, simply as a matter 
C. BELLERopHoN, G. \& H.

C. bellerophon, G. \& H., op. cit., p. 132, fig. 13.

REPTESChaRELLA, d'Orb. R. Heermannit, G. \& H.

R. Heermannii, G. \& H. ; op. cit., p. 137, fig. 20.

R. Plana, G. \& H.

R. plana, G. \& H. ; op. cit., p. 137, fig. 21.

PHIDOLOPORA, G. \& II. P. LABIATA, G. \& H.

Phidolopora labiata, G. \& H. ; op. cit., p. 138, fig. 21.

REPTOPORINA; d'Orb. R. eustomata, G. \& H.

R. customata, G. \& H. ; op. cit., p. 144, fig. 26.

REPTESCHARELLINA, d'Orb. R. DISPARILIS, G. \&. H.

R. disparilis, G. \& H. ; op. cit., p. 147, fig. 29.

? R. Heermannit, G. \& H.

? R. Heermannii, G. \& H.; op. cit., p. 147, fig. 30 .

R. Cornuta, G. \& H.

R. cornuta, G. \& H. ; op. cit., p. 147, fig. 31 .

SIPHONELLA, Hagen. S. MULTIPORA, G. \& H.

S. multipora, G. \& H.; op. cit., p. 154, tig. 38 .

MEMBRANIPoRA, Blainv. M. CaLifornica, G. \& H.

M. Californica, G. \& H.; op. cit., p. 160, fig. 46.

of convenience; though I am satisfied that that author's divisions are eminently artificial. A more extensive acquaintance with the subject, based on a study of living as well as fossil forms, has satisfied me of the fallacy of his system, founded on partial characters derived from the skeletons alone. 
M. Barbarensis, G. \& H.

M. Barbarensis, G. \& H.; op. cit., p. 160, fig. 47.

idMONEA, Lamx. I. Californica, Con.

Idmonea Californica, Con.; Proc. Phil. Acad., 1855, p. 441.

Id., G. \& H. ; op. cit., p. 168, fig. 56.

SEMITUBIGERA, d'Orb. S. TUBA, G. \& H.

S. $t u b a$, G. \& H.; op. cit., p. 169, fig. 57.

ENTALOPHORA, Lamx. E. punctulata, G. \& H.

E. punctulata, G. \& H. ; op. cit., p. 171, fig. 61 .

LICHENOPORA, Defr. L. CaLifornica, Con.

L. Californica, Con.; Proc. Phil. Acad., 1855, p. 441.

Id., G. \& H. ; op. cit., p. 176, fig. 68.

\section{RADIATA.}

CLYPEASTER, Lam. C. GABBIr, Rém.

Clypeaster Gabbii, Rém.; Proc. Cal. Acad., 1863, p. 63.

Id., Gabb; Pal. Cal., Vol. 2, p. 12, fig. 64, $64 a$.

Miocene, San Pablo, Martinez, Walnut Creek, and vicinity of Mount Diablo.

echinarachivius, Van Phels. E. Brewertanus, Rém.

E. Brewerianus, Rém. ; loc. cit., 1863, p. 53.

Id., Gabb; Pal. Cal., Vol. 2, pl. 12, fig. 65.

Miocene, Walnut Creek.

SCUTELLA, Lam. S. Gibisir, Rém.

Scutella Gibbsii, Rém. ; loc. cit., p. 13.

Id., Gabb; Pal. Cal., Vol. 2, pl. 13, fig. 66.

Miocene, near Buena Vista Lake. 


\section{S. STRIATULA.}

S. striatula, P. R. R. Rep., Vol. 7, pl. 19, fig. 1.

Post-Plioeene, Santa Barbara, San Pedro, Seven Mile Beaeh, near San Fran. ciseo. Living.

S. interlineata, Stimpson.

Scutella interlineata, St. ; P. R. R. Rep., Vol. 5, p. 153, pl. 4, fig. 30.

Plioeene, Seven Mile Beaeh, near San Franeiseo.

\section{ASTRODAPSIS, Con. A. ANTISELLI, Con.}

Astrollapsis antiselli, Con.; P. R. R. Rep., Vol. 7, p. 196, pl. 10, fig. 1, 2. Miocene, near Buena Vista Lake.

\section{A. Whitneyi, Rém.}

Astrodapsis Whitneyi, Rém.; Proc. Cal. Aead., 1863, p. 52.

Id., Gabb; Pal. Cal., Vol. 2, pl. 13, fig. 67.

- Plioeene, Kirker's Pass.

\section{A. тumidus, Réın.}

A. tumidus, Rém.; Proe. Cal. Aead., 1863, p. 52.

Id., Gabb; Pal. Cal., Vol. 2, pl. 13, fig. 68.

\section{ASTERIAS, Linn. A. Rémondr, Gabb.}

Asterias Rémondi, Gabb; Pal. Cal., Vol. 2, p. 37, pl. 13, fig. 69.

Miocene, Star Fish Point, West of Martinez. 


\section{SPECIES}

NOT YET ENCOUNTERED OR RECOGNIZED.

Callianassa, Leach. C. Oregonensis, Dana.

Callianassa Oregonensis, Dana; Wilkes's Exped., p. 722, pl. 17, fig. 3.

Miocene, Oregon.

MUREX, Linn. M. Fragilis, Trask.

Id., Trask; Proc. Cal. Acad. Nat. Sci., 1855, p. 42.

Post-Pliocene, Sunta Barbara.

FUSUS, Lam. F. arctatus, Con., sp.

Colus arctatus, Con.; P. R. R. Rep., Vol. 5, p. 329, pl. 8, fig. 76.

Fusus arctatus, Meek; Smithsonian Check List, No. 749.

F. Barbarknsis, Trask.

Id., Trask; Proc. Cal., Acad. Nat. Sci., 1855, p. 42.

Post-Pliocene, Santa Barbara.

F. Robustus, Trask.

Id., Trask; loc. cit.

Post-Pliocene, Santa Barbara.

F. Rugosus, Trask.

Id., Trask; loc. cit.

Post-Pliocene, Santa Barbara.

BUSYCON. ? B. Oregonensis, Con., sp.

Fusus id, Con.; Amer. Jour. Sci., 1848, p. 433, fig. 13.

Sycotypus id., Con. ; Jour. Conch., 1865, p. 151.

Busycon? id., Meek; Sm. Check List, No. 743.

Miocene, Astoria. 
PRISCOFUSUS, Con. P. corpulentus, Con.

Fusus corpulentus, Con.; Wilkes's Exped., p. 728, pl. 20, fig. 4. Priscofusus id., Con.; Jour. Conch., 1865, p. 150.

Miocene, Astoria.

\section{P. Devinctus, Con.}

Buccinum devinctum, Con.; Wilkes's Exped., p. 728, pl. 20, fig. 2. Priscofusus id., Con. ; Jour. Conch., 1865, p. 150.

Miocene, Astoria.

$$
\text { P. medialis, Con. }
$$

Cerithium mediale, Con.; Wilkes's Expcd., p. 728, pl. 20, fig. 1.

P. medialis, Con.; Jour. Conch., 1865, p. 150.

Miocene, Astoria.

$$
\text { P. nodiferus, Con. }
$$

Indet., Con.; Wilkes's Exped., pl. 20, fig. 12.

P. nodiferus, Con.; Jour. Conch., 1865, p. 150.

Miocene, Astoria.

\section{P. Oregonensis, Con.}

Indet., Con. ; Wilkes's Exped., pl. 20, fig. 10, 11. P. Oregonensis, Con.; Jour. Conch, 1865, p. 150. Miocene, Astoria.

Pledrotoma, Lam. P. transmontana, Con.

P. transmontana, Con.; P. R. R. Rep., Vol. 5, p. 328, pl. 7, fig. 69. Turris id., Meek; Smithsonian Check List, No. 693.

Miocene, Ocoya Creek.

PURPURA, Brug. P. petrosa, Con. sp.

Stramonita petrosa, Con.; P. R. R. Rep., Vol. 5, p. 327, pl. 6, fig. 47 Purpura id., Meek; Smithsonian Check List, No. 663.

Galeodea, Link. G. petrosa, Con.

G. petrosa, Con.; Smithsonian Eocene Check List, No. 471.

"Oregon." 
The above list gives no means of determining where this species was described; and I can find no form to which to refer it, unless it be one of the succeeding, both of which have been placed under the same specific name.

\section{CASSIDEA, Swains. C. ? Bilirata, Con., sp.}

Semicassis? biliratus, Con.; Sm. Check List (1866), No. 472.

Doliopsis id., Con.; Jour. Conch., 1865, p. 150.

Dolium petrosum, Con.; (pars) Wilkes's Exped., p. 727, pl. 19, fig. 4.

Miocene, Oregon.

\section{C. ? Petrosa, Con., sp.}

Semicassis petrosus, Con.; Sm. Check List, No. 474.

Doliopsis id., Con.; Jour. Conch., 1865, p. 150.

Dolium id., Con.; Wilkes's Exped. (pars), p. 727, pl. 19, fig. 3, 5.

Miocene, Oregon.

FICOPSIS, Con. F. modesta, Con., sp.

Pymula modesta, Con.; Am. Jour. Sci., 1848, p. 433, fig. 12.

Sycotypus id., Con.; Jour. Conch., 1865, p. 151.

Ficus id., Meek; Sm. Check List, Miocene, No. 637.

Ficopsis id., Con.; Sm. Check List, Eocene, No, 479.

Miocene, Oregon.

FICUS, Bolt. F. Ocoyanus, Con., sp.

Sycotypus Ocoyanus, Con.; P. R. R. Rep., Vol. 5, p. 329, pl. 7, fig. 72.

Ficus id., Meek; Sm. Check List, Miocene, No. 635.

Miocene, Ocoya Creek.

NATICA, Brug. N. Geniculata, Con.

Id., Con. ; P. R. R. Rep., Vol. 5, p. 328, pl. 7, fig. 67.

Miocene, Ocoya Creek.

It is not impossible that this may prove to be identical with Agasoma gravida, but the figure in the Pacific Railroad Report is so very uncharacteristic, that I have been unable to arrive at a satisfactory opinion on the subject.

PAL. VOL. IT. -16 
LUnatia, Gray. L. Oregonensis, Con.

L. Oregonensis, Con.; Jour. Conch., 1865, p. 151.

Sigaretus scopulosus, Con.; (pars) Wilkes's Exped., pl. 19, fig. 6, b, c. Miocene, Oregon.

SINUM, Bolt. S. scopulosum, Con., sp.

Sigaretus scopulosus, Con.; Wilkes's Exped., p. 727, pl. 19, fig. 6 (exclus. b,c).

Catinus scopulosus, Con.; Jour. Conch., 1865, p. 151.

Catinus scopulosus, Con.; Sm. Check List, No. 469.

Catinus scopulosus, Con.; (in Meek), Miocene Chcck List.

Mr. Meek calls this shell Catinus, by request of Mr. Conrad, but under protest, and quotes Sinum as the more correct name.

Miocenc, Oregon.

TURBONILla, Risso. T. Papillosa, Trask, sp.

Chemnitzia papillosa, Trask; Proc. Cal. Acad, 1855, p. 41.

Turbonilla papillosa, Mcek; Sm. Check List, No. 592.

Post-Pliocene, Santa Barbara.

Cancellaria, Lam. C. ? Oregonensis, Con.

? C. Oregonensis, Con.; Jour. Conch., 1865, p. 151.

Indet., Con.; Wilkes's Exped., pl. 20, fig. 8.

Miocene, Astoria.

CERITHIOFsis, F'bs. \& Hanley. ? C. Oregonensis, Con.

Cerithiopsis? Oregonensis, Con.; Jour. Conch., 1865, p. 151.

Indet., Con.; Wilkes's Exped, pl. 20, figs. 13, 14.

Miocene, Astoria.

TURRITELLA, Lam. T. Ocoyana, Con.

T. Ocoyana, Cor.; P. R. R. Rep., Vol. 5, p. 329, pl. 8, fig. 72.

Miocene, Ocoya Creek.

Mesalia, Gray. M. arenicola, Con.

M. arenicola, Con.; Sm. Check List, Eocene, No. 326.

I know nothing of this shell, except that I find it quoted as above and referred to "Oregon." 
Trochita, Schum. T. Dregoana, Con.

T. Diegoana, Con.; P. R. R. Rep., Vol. 5, p. 327, pl. 5, fig. 42.

Miocene, San Diego.

CRYPTA, Humph. C. rostralis, Con.

C. rostralis, Con.; Jour. Conch., 1865, p. 151.

Indet, Con.; Wilkes's Exped., 1850, pl. 19, fig. 11.

Probably the same as C. rostriformis, Gld.; Proc. Bost. N. Hist. Soc., 1846, p. 160, and C. rostriformis, Gld. ; Wilkes's Exped., Mollusca, p. 375, pl. 32, fig. 482.

Miocene, Astoria.

VANIK0R0, Quoy \& Gaim. ? V. Diegoana, Con., sp.

Narica Diegoana, Con. ; P. R. R. Rep., Vol. 5, p. 326, pl. 5, fig. 39.

Vanikoro Diegoana, Meek; Check List, Miocene, No. 498.

Miocene, San Diego.

CEMORIA, Leach. C. crucibuliformis, Con.

Diadora crucibuliformis, Con.; Proc. Phil. Acad., 1855, p. 267.

Id., Con.; P. R. R. Rep., Vol. 6, p. 73, pl. 5, fig. 23.

Cemoria id., Con.; in Meek's Check List, No. 467.

Post-Pliocene, Santa Barbara. Living.

DENTALIUM, Linn. D. substriatum, Con., sp.

Teredo substriata, Con.; Wilkes's Exped., 1850, p. 728, pl. 20, fig. 7.

Dentalium substriatum, Woodw.; Brit. Assn. Rep., 1856.

Dentalium substriatum, Con.; Jour. Conch., 1865, p. 151.

Miocene, Astoria, Oregon.

ACTE0N, Montf. A. ELLIPTICUs, Trask, sp.

Tornatella elliptica, Trask; Proc. Cal. Acad., 1855, p. 42.

Actcoon elliptica, Meek; Check List, No. 434.

Post-Pliocenc, Santa Barbara.

CYLICHNa, Loven. C. Oregona, Con.

C. Oregona, Con.; Jour. Conch., 1865, p. 151.

Bulla petrosa, Con.; Wilkes's Exped., p. 727, pl. 19, fig. 8.

Miocene, Astoria. 


\section{C. petrosa, Con.}

C. petrosa, Con. ; Jour. Conch., 1865, p. 151.

Bulina petrosa, Con.; Am. Jour. Sci., 1848, p. 433, fig. 11.

Miocene, Oregon.

Bulla, Linn. B. Jugularis, Con.

B. jugularis, Con.; P. R. R. Rep., Vol. 5, p. 328, pl. 7, fig. 62.

Miocene, Ocoya Creek.

\section{ACEPHALA.}

ENSIS, Schum. E. curtus, Con.

Solen curtus, Con.; Am. Jour. Sci., 1848, p. 433, fig. 13.

Ensis curtus, Con.; Jour. Conch., 1865, p. 152.

Plectosolen curtus, Con.; Sm. Check List, No. 239.

Miocene, Oregon.

SAXICAVA, Fleur. S. ABRUPta, Con.

S. abrupta, P. R. R. Rep., Vol. 5, p. 324, pl. 3, fig. 25.

Post-Pliocene, San Pedro.

MYA, Linn. M. Montereyana, Con.

Mya Montereyana, Con.; P. R. R. Rep., Vol. 6, p. 70, pl. 2, fig. 4 Probably = Cryptomya Californica.

M. subsinuata, Con.

Mya subsinuata, Con.; P. R. R. Rep., Vol. 7, p. 72, pl. 2, fig. 5. Probably also $=$ Cryptomya Californica .

Corbula, Brug. C. Diegoana, Con

Corbula Diegoana, Con.; P. R. R. Rep., Vol. 5, p. 322, pl. 3, fig 16. Post-Pliocene, San Diego. 


\section{TERTIARY FOSSILS. \\ C. Evansana, Shum.}

Corbula Evansana, Shumn.; Tr. St. Louis Acad., Vol. 1, p. 120.

Miocene, Port Orford, Oregon.

THRACIA, Leach. T. mactropsis, Con.

Thracia mactropsis, Con.; P. R. R. Rep., Vol. 6, p. 69, pl. 2, fig. 3.

"Monterey County, California."

PANDORA, Brug. P. Bilirata, Con.

P. bilirata, Con.; P. R. R. Rep., Vol. 6, p. 73, pl. 5, fig. 25.

Santa Barbara.

maCTRA, Linn. M. Gabiotensis, Con.

Mactra Gabiotensis, Con.; P. R. R. Rep., Vol. 7, p. 194, pl. 7, fig. 3.

Miocene, Gaviota Pass.

LUtraria, Lam. L. transmontana, Con.

Lutraria transmontana, Con.; P. R. R. Rep., Vol. 7, p. 194, pl. 5, fig. 6.

Los Angeles County.

TELlina, linn. T. Diegoana, Con.

Tellina Diegoana, Con.; P. R. R. Rep., Vol. 5, p. 323, pl. 3, fig. 28.

San Diego.

T. obruta, Con.

Tellina obruta, Con.; Am. Jour. Sci., 1848, p. 436, fig. 6.

Miocene, Oregon.

T. albaria, Con.

Tellina albaria, Con. ; Wilkes's Exped., p. 725, pl. 18, fig. 5.

Miocene, Oregon.

T. Bitruncata, Con.

Tellina bitruncata, Con.; Wilkes's Exped., p. 725.

Oregon. 
ARCOPAGIA, Leach. A. Unda, Con.

Arcopagia unda, Con.; P. R. R. Rep., Vol. 7, p. 192, pl. 4, fig. 34.

Miocene, Santa Barbara, Estrella.

MACOMA, Leach. M. Ocoyana, Con., sp.

Tellina Ocoyana, Con.; P. R. R. Rep., Vol. 5, p. 329, pl. 7, fig. 75. Miocene, Ocoya Creek.

\section{Arctata, Con., sp.}

Tellina arctata, Con.; Wilkes's Exped., p. 725, pl. 18, fig. 3.

Miocene, Orcgon.

VenUs, Linn. V. Pajaroensis, Con.

Venus Pajaroensis, Con.; P. R. R. Rep., Vol. 7, p. 192, pl. 4, fig. 1, 2. Pajaro, near San Juan Bautista.

CHIONE, Megerle. C. Angustifrons, Con.

Venus angustifrons, Con.; Wilkes's Exped, p. 724, pl. 17, fig. 11.

Dione angustifrons, Con.; Jour. Conch., 1865, p. 152.

Miocene, Astoria.

\section{C. vespertina; Con., sp.}

Cytherea vespertina, Con.; Am. Jour. Sci., 1848, p. 432, fig. 9.

Dione vespertina, Con.; Jour. Conch., 1865, p. 152.

Miocene, Oregon.

MERETRIX, Lam. M. UNIOMERIS; Con.

Meretrix uniomeris, Con. ; P. R. R. Rep., Vol. 5, p. 323, pl. 3, fig. 20. Miocene, Tres Pinos, Monterey County.

\section{DECISA, Con.}

Meretrix decisa, Con.; P. R. R. Rep., Vol. 5, p. 323, pl. 3, fig. 27. Miocene, Ocoya Creek. 


\section{Tularana, Con.}

Meretrix Tularana, Con.; P. R. R. Rep., Vol. 5, p. 323, pl. 3, fig. 22. ? Miocene, San Emidio.

PACHYDESMa, Con. P. Inezana, Con.

Pachydesma Inczana, Con.; P. R. R. Rep., Vol. 7, p. 197, pl. 5, fig. 204.

Miocene, Santa Inez Mountains.

DOSINIA, Scop. ? D. LONGUla, Con.

Dosinia longula, Con.; Proc. Phil. Acad., 1856, p. 313.

Id., Con.; P. R. R. Rep., Vol. 6, p. 71.

$I d$., Con. ; P. R. R. Rep., Vol. 7, p. 193, pl. 7, fig. 2.

Miocene, Salinas Valley.

This shell certainly does not look like a Dosinia.

D. montana, Con.

Dosinia montana, Con.; P. R. R. Rep., Vol. 7, p. 194, pl. 6, fig. 4.

Miocene, same locality.

D. subobliqua, Con.

Dosinia subobliqua, Con.; P. R. R. Rep., Vol. 7, p. 194, pl. 6, fig. 5. Miocene, same locality.

TAPES, Megerle. T. Montana, Con.

Tapes montana, Con.; P. R. R. Rep., Vol. 7, p. 192, pl. 5, fig. 3 and 5. San Buenaventura.

T. Inezensis, Con.

T. Inezensis, Con.; P. R. R. Rep., Vol. 7, p. 192, pl. 7, fig. 1 . Miocene, Santa Incz Mountains.

Petricola, Lam. P. Pedroana, Con.

Petricola Perlroana, Con.; P. R. R. Rep., Vol. 5, p. 324, pl. 3, fig. 24.

San Pedro. 
CARDiUM, Linn. C. modestum, Con.

C. modestum, Con.; P. R. R. Rep., Vol. 5, p. 322, pl. 3, fig. 15. San Diego.

LUCINA, Brug. L. FIBRosa, Shum.

Lucina fibrosa, Shum; Tr. St. Louis Ácad., Vol. 1, p. 120.

Cyclas fibrosa, Con. ; Jour. Conch., 1865, p. 153.

Port Orford, Oregon.

L. Permacra, Con.

Cyclas (Lucina) permacra, Con.; P. R. R. Rep., Vol. 7, p. 192, pl. 7, tig. 4. Santa Monica.

L. Estreluana, Con.

Cyclas (L.) Estrellana, Con.; P. R. R. Rep., Vol. 7, p. 192, pl. 6, fig. 6. Miocenc, Estrella.

\section{MYTILUS, Linn. M. Pedroanus, Con.}

M. Pedroanus, Con.; P. R. R. Rep., Vol. 5, p. 325, pl. 5, fig. 40.

? Post-Pliocene, San Pedro.

\section{Inezensis, Con.}

M. Inezensis, Con.; P. R. R. Rep., Vol. 7, p. 194, pl. 8, fig. 2, 3. Miocene, Santa Inez Mountains.

Modiola, Lam. M. contracta, Con.

Modiola contracta, Con.; P. R. R. Rep., Vol. 5, p. 325, pl. 5, fig. 25.

"Sixteen miles south of Tres Pinos, Monterey County," on the San Benito.

PERNA, Brug. P. Montana, Con.

Perna montana, Con.; P. R. R. Rep., Vol. 7, p. 195.

San Buenaventura. 
ARCA, Linn. . A. Devincta, Con.

Arca devincta, Con.; Wilkes's Exped., p. 726, pl. 18, fig. 10.

Anomalocardia id., Con.; Jour. Conch., p. 153.

? A. microdonta, Con.; see note under that species in preceding list.

Miocene, Oregon.

\section{A. Obispoana, Con.}

Arca Obispoana, Con.; P. R. R. Rep., Vol. 7, p. 192, pl. 5, flg. 1. San Louis Obispo.

\section{A. Congesta, Con.}

Arca congesta, Con. ; P. R. R. Rep., Vol. 6, p. 72, pl. 2, fig. 10. "California."

LIMOPSIS, Sassi. L. Nitens, Con,

Pectunculus nitens, Con.; Wilkes's Exped., p. 726, pl. 18, fig. 8.

Limopsis id., Con.; Jour. Conch., 1865, p. 163.

Miocene, Oregon.

\section{Oregonensis, Con.}

Indet., Con. ; Wilkes's Exped., pl. 18, fig. 11.

Limopsis Oregonensis, Con.; Jour. Conch., 1865, p. 153.

Miocene, Oregon.

LEDA, Schum. L. Oregona, Shum.

Leda Oregona, Shum.; Trans. St. Louis Acad., Vol. 1, p. 121.

Neilo id., Con.; Jour. Conch., 1865, p. 153.

Nuculana id., Con.; Check List Eocene, No. 49.

Miocene, Oregon.

L. penita, Con., sp.

Nucula penita, Con.; Am. Jour. Sci., 1848, p. 433, fig. 10.

Neilo penita, Con.; Jour. Conch., 1865, p. 153.

Nuculana penita, Con.; Check List Eocene, No. 51.

Miocenc, Oregon.

PAL. YOL. II. -17 


\section{Willamettensis, Sham.}

Leda Willamettensis, Shum.; Tr. St. Louis Acad., Vol. 1, p. 121. Neilo Willamettensis, Con.; Am. Jour. Conch, 1865, p. 153. Nuculana Willamettensis, Con.; Check List Eocene, No. 59. Miocene, Oregon.

NEILO, II. \& A. Ad. N. ABRUpta, Con.

Nucula abrupta, Con.; Am. Jour. Sci., 1848, p. 433, fig. 3. Ncilo abrupta, Con.; Jour. Conch., 1865, p. 153.

Miocene, Oregon.

PECTEN, Linn. P. Coosensis, Shum.

P. Coosensis, Shum.; Tr. St. Louis Acad., Vol. 1, p. 122.

Miocene, Coos Bay, Oregon.

Compare $P$. propatulus, Con.

\section{P. Deserti, Con.}

P. Deserti, Con. ; P. R. R. Rep., Vol. 5, p. 325, pl. 5, fig. 41. Pliocene, Colorado Desert.

\section{P. Nevadanus, Con.}

P. Neradanus, Con.; P. R. R. Rep., Vol. 5, p. 329, pl. 8, fig. 27. Miocene, Ocoya Creek.

\section{P. Mеeкi, Con.}

P. Meekii, Con.; P. R. T. Rep., Vol. 7, p. 190, pl. 1, fig. 1. San Rafael Hills.

$$
\text { P. Discus, Con. }
$$

P. discus, Con.; P. R. R. Rep., Vol. 7, p. 190, pl. 3, fig. 1. Santa Incz.

\section{P. magnolia, Con.}

P. magnolia, Con.; P. R. R. Rep., Vol. 7, p. 191, pl. 1, fig. 2. Santa Inez. 
ostrea, Linn. O. subjecta, Con.

Ostrea subjecta, Con.; P. R. R. Rep., Vol. 7, p. 193, pl. 2, fig. 3.

Sierra Monica, Miocene?

\section{O. Panzana, Con.}

Ostrea Panzana, Con.; P. R. R. Rep., Vol. 7, p. 193, pl. 2, fig. 4. Panza Valley and Estrella, Miocene.

TEREBRATULA, Llhyd. ? T. nITens, Con.

Terebratula nitens, Con.; Wilkes's Exped., p. 726, pl. 19, fig. 1.

Rhynconella nitens, Con.; Jour. Conch., 1865, p. 154.

Miocene, Astoria.

\section{RADIATA.}

GALERITES. G. Oregonensis, Dana.

Galerites Oregonensis, Dana; Wilkes's Exped., p. 729, p1. 21, fig. 5, 6. Miocene, Oregon.

\section{ADDITIONS AND CORRECTIONS.}

Since the preceding pages were stereotyped, the following additions and corrections have been obtained from my friends Messrs. Tryon and Stearns, who have examined the proof sheets. I have also detected two or thrce omissions, which are here corrected.

Trophon squamulifer, Cpr. Found by Mr. Stearns, living at Monterey and Catalina Island; common.

Neptunea linata, Mart., Alaska; a large series has bcen eompared by Stearns and Morse with $N$. decemcostata, and they eonsider them identical; circumboreal.

Margarita salmonea, Cpr. "Is not worth a variety distinction from pupilla." (Stearns.)

Clypidelua bimaculata, Dall. Is now considered by Stcarns and Carpenter, to be identical with callomarginata, after a comparison of a large suitc of shells and animals. 


\section{ZIRPHÆA, Leach.}

Z. Dentata, Gabb.

Z. dentata, Gabb; Pal. Cal., Vol. 2, p. 18, pl. 3, fig. 31 a.

Pliocene, Kirker's Pass.

\section{MACOMA, Leach.}

M. calcarea, Chemn., sp.

Tcllina calcarea, Chemn.; Conch. Cab., Vul. 6, p. 140, fig. 136.

T. inconspicua, Brod. and Sby ; Zool. Jour., Vol. 4, p. 363.

Sanguinolaria Californica, Con.; Jour. Phil. Acad., 1 Ser., Vol. 7, p. 231, pl. 17, f. 11.

Tellina lata, Gmel.; Syst. Nat., p. 3237.

T. proxima, Brown; Zool. Becchey's Voy., p. 154, pl. 44, fig. 4.

T. Pedroana, Con.; Pacific R. R. Rep., Vol. 5, p. 323, pl. 3, fig. 17.

T. sordida, Couth.; Bost. Jour., Vol. 2, p. 59, pl. 3, fig. 11.

$T$. tcnera, Lcach; Appendix to Ross's Voyage.

Macoma Pcdroana, Gabb; Vol. 2, p. 94.

Mac. inconspicua, Cpr.; Brit. Assn. Rep., 1863.

Circumboreal; fossil in the Post Pliocene of San Pedro.

ARCA, Lam.

A. sulcicosta, Gabb.

Arca sulcicosta, Gabb ; Pal. Cal., Vol. 2, p. 31, pl. 9, fig. 53, $53 \boldsymbol{a}$. Pliocene, Mark West Creek, near Sta. Rosa.

\section{MORRISIA, Davidson.}

M. Hornil, Gabb.

Morrisia Hornii, Gabb; Proc. Phil. Acad., 1861, p. 371.

Id., Gabb ; Pal. Cal., Vol. 2, p. 36, pl. 12, fig. 63.

Post Pliocene, Sta. Barbara. 


\title{
PALEONTOLOGY OF CALIFORNIA.
}

\author{
V 0 I. I I.
}

SECTION II.

CRETACEOUS FOSSILS.

P A R T I.

DESCRIPTION OF NEW SPECIES.

(CONTINUED FROM VOL. I.) 



\section{CRUST A CEA.}

Crostace aN remains are extremely rarc in the California Cretaceous; fragments if but three species having yet been encountered. Pl. 19, fig. 1, represents a carapace of a small crab, a unique specimen from Martinez, discovered by $\mathrm{Mr}$. Mathewson, in a coarse-grained gray sandstone. Figures 2, $a-f$, are three fragments of claws, remarkable for their long, slender form. Dr. Stimpson concur's with me in the belief that they all belong to the same species, though they vary much both in size and shape. They are from a dense gray claystone, associated with Ptiloteuthis foliatus and Diptychoceras lavis, from the vicinity of Cottonwood Creek, Shasta County.

\section{CALLIANASSA, Leach.}

\section{Stimpsonit, Gabb.}

Pl. 19, Fig. 3.

(Callianassa Stimpsonii, Gabb ; Pal. Cal., Vol. 1, p. 57, pl. 9, fig. 1, a, b. c.)

Figure $1 b$, above quoted, was unfortunately so misrepresented by the artist as not only to be unrecognizable, but also to give an entirely incorrect idea of the shape. This was due to the original drawing representing the two upper angles broken away, and the lithographer having substituted a curved line in the place of the broken one in my drawing. The present figure is from the same sketch, with the sole addition of the probable outline being restored. 


\title{
MIOLLUSCA.
}

\section{CEPHALOPODA.}

\author{
Ptiloteuthis, N. Gen.
}

Elongate, subovate, very thin, anterior end broadly angulated, no mid-rib; slipper either minute or wanting. Surface marked by numerous, irregular, small wrinkles, which radiate backwards and outwards, partly from the anterior end and partly from an imaginary median line.

The present anomalous form cannot be confounded with any previously-described genus. Belotenthis and Phylloteuthis are narrow in front, and enlarge more or less behind, and both have a mid-rib. Beloteuthis has straight radiating lines, all divcrging from the anterior end, and these are usually crossed by concentric lines or undulations; Phylloteuthis has a very narrow anterior end, a mid-rib, and wellmarked, straight, rigid, lateral striæ diverging at a broad angle from the mid-rib alone. Coccoteuthis has a thick substance, and the surface is strongly pustulated. Ptiloteuthis is very thin; the lines or wrinkles, about twicc as wide apart as the thickness of the shell-substance, branch and anastomose among themselves, and radiate in part from the anterior end, but in part diverge from a central line occupying the proper position for a mid-rib, but only marked by the change in the direction of the striæ. The anterior end terminates in an angle of about $60^{\circ}$, and the slipper, if it ever existed, was so small as to be barely more than rudimentary.

\section{P. Foliatus, n. s.}

Pl. 19, Fig. 4.

Shell thin, elongated, subovate; anterior end angulated, the sides rounding and rapidly widening, merging into the lateral margins, which are broadly convex, nearly parallel; posterior 
end unknown, the terminal portion being very much broken in the only specimen I have seen. From the median line, the position of the mid-rib in most of these shells, the wrinkles diverge backwards and outwards at an angle of about $25^{\circ}$, curving more outwardly as they approach the margin; these wrinkles are acute on the dorsal face of the shell, and inclose concave interspaces of very variable length; the longest lines, which originate at the anterior end of the shell, reach the margins about midway between the two extrenities.

Length, 3 inehes + ; width, 1.2 inehes in the middle; the total length seems to have been from 3.3 to 3.5 inches. Thiekness apparently not moro than .01 ineli, as exposed by several fraetures.

Locality: Shasta Group,* Cottonwood Creek, Shasta County. A single specimen in my eabinet, found by Mr. Mathewson.

\section{Belemanites impressus, Gabb.}

This is the only eretaeeous Belemnite so far diseovered in California. It is one of the most charaeteristie fossils of the Shasta Group, and besides the localities mentioned in the report of the Survey, Vol. 1, it has been found at various plaees on the east side of the Northern Coast Range, more especially in Colusa County, and elsewhere east of Clear Lake.

* For reasons which will be detailed in their proper plaee, I eonsider the group, for which I liere propose this name, to be the equivalent, or at least the nearest representative, of the $\mathrm{Neoeomien}$. It has been herctofore included, for want of positive grounds of separation, as a provisional member of the "Division A," of the California Cretaceous. I shall, further, in this paper, use the term Martinez Group, for the upper portion of "Division A" of the California Reports; the Chieo Group, for those beds of which Chieo Creek, Penee's Ranch, and Tusean Springs are typieal loealities; and shall eall "Division B" the Tejon Group, these loealitics being the points where the respeetive formations are most strongly developed.

PAI. VOL. II. -18 


\title{
AMMONITES, Brug.
}

\author{
A. Brewerit, Gabb.
}

Pl. 20, Fig. 5; Pl. 19, Fig. $5 a, b, 6,6 a$.

(Ammonites Brewerii, Gabb; Pal. Cal., Vol. 1, p. 62, pl. 10, fig. 7.)

(A. Ncxberryanus, Gabb; (pars) loc. cit., Pl. 10, fig. 6.)

This shell was originally deseribed from several imperfect easts in sandstone, showing nothing but the surfaee eharaeters, and not all of those in a perfeetly satisfaetory manner. Subsequent researeh has plaeed me in possession of abundant material for redescribing the species.

Shell diseoidal, eompressed; whorls inereasing in size with moderate rapidity, eaeh whorl embraeing about one-half of the preceding one; sides flattened, eonverging eonvexly towards the dorsum, whieh is narrowly rounded; umbilical margin of the whorl abruptly truncated at nearly a right angle. Mouth semielliptical, the dorso-ventral diameter twiee the transverse, the ventral emargination, produeed by the encroaehment of the preceding volution, being about one-fifth of the greater diameter. Umbilicus nearly one-fourth the diameter of the shell. Surfaee variable from nearly smooth, or marked only by sin uous strix, to strongly eostate, the striæ assuming the eharaeter of irregular ribs, most marked near, and on the dorsnm.

Septum: Dorsal lobe small, bifureate a little below the middle, and bearing two points in the noteh; each branch earrying two spurs on the outer side; above the bifurcation are a large and a small spur on the main lobe. Dorsal saddle broad, divided into two unequal parts, the lower of whieh is much more deeply subdivided than the upper. Superior lateral lobe longer than the dorsal, trifurcate, the lateral branch on the upper side arising somewhat higher than the eorresponding one on the other side, and being much more subdivided; above the origin of these 
branches are two spurs on each side; these, as throughout the whole septum, are more complex on the dorsal than on the ventral side of the lobe. Lateral saddle similar to the dorsal, but smaller. Inferior lateral lobe about as long as the dorsal, resembling in shape the superior lateral, but not half so complex; the inferior lateral branch having diminished to a mere spur. Below this, to the umbilical angle, are three lobes and three saddles, presenting the fundamental characters of the laterals, but rapidly simplifying.

This description of the septum would apply very nearly to that of A. Hoffman$n i i$, but in that species the lobes are broader, more symmetrical on the upper and lower sides, the saddles are proportionally larger, and the diminution in size and complexity of the lobes from the superior lateral is more marked and rapid.

The illustration given in Pal. Cal., Vol. 1, pl. 10, fig. 6, is of a young specimen from Cottonwood Creek, Shasta County.

- Locality: Shasta Group, Cottonwood Creek.

\section{A. Traskit, Gabb.}

Pl. 19, Fig. 7, $a, b$.

(A. Traskii, Gabb ; Pal. Cal., Vol. 1, p. 63, pl. 11, fig. 10; pl. 12, fig. 11.)

Young specimens of this shell have so many points of difference from the adult that they might be mistaken for a different species. The adult has a mouth nearly as broad as long, the sides of the whorl being strongly arched. A specimen a little over two inches in diameter, shows a section nearly identical with that of A. Hoffmannii, Pl. 11, fig. 13 , while a still smaller one before me, 1.3 inch in diameter, is as flat on the sides as $A$. Brewerii; and the ribs, which are strongly sinuous on the sides, become obsolete at the dorso-lateral margin, the dorsum being very much flattened and smooth.

This species is not rare at Cottonwood Creek, Shasta Group.

\section{A. Hoffmannit, Gabb.}

$$
\text { Pl. 20, Fig. 8, } a \text {. }
$$

(A. Hoffmannii, Gabb; Pal. Cal., Vol. 1, p. 65, pl. 11, 12, fig. 13, a, b, c.)

A variety of this shell was collected by Mr. Mathewson at Cottonwood Creek, in which the whorls are much thicker than in the form described; the aperture 
being exactly as wide transversely, as from the dorsum to the umbilical margin. The periodieal constrictions on the east.figured, are about as numerous on these specimens as on the flattcr forms, the thickening of the shell at those points showing itself as a faint rib on the outside; a specimen before me has eight on one volution. The other ribs, mentioned in the deseription and shown in the figure quoted above, are entirely absent in some cases. No differences exist in the septum.

\section{A. Batesir, Trask.}

Pl. 20, Fig. 9, $a$; Pl. 21, Fig. 10, $\alpha, b$.

(A. Batesii, Trask; Proe. Cal. Acad., 1855, p. 40.)

(Id., Gabb ; Pal. Cal., Vol. 1, p. 67, pl. 13, fig. 16, $16 a$, b.)

'The largest known, most widely diffused, and one of the most variable Ammonites of California.

The form described by Dr. Trask, and used by me as the typical form of the species, is many whorled, the volutions nearly eircular in seetion, increasing very slowly in size, and barely more than in contact. In this form the surface is usually ornamented by simple, linear ribs, with fine revolving lines in the interspaces. Another variety was mentioned, in which the whorls increase much more rapidly in sizc, several specimens having been found of over a foot in diameter. A third variety has since been obtained, in whieh, in the young state, up to an inch, or an inch and a half in diameter, the whorls are broader than high, the dorsum broadly rounded, and the unbilicus occupies more than lialf the diameter of the shell, is funnel-shaped, very deep, and is bordered by a sharp angle, the surface between this angle and the suture being flat. As the shell in this variety grows older the angle disappears, the flattening gradually rounds out, and the older shell assumes the normal form of the species, except that it bears an occasional well-marked, rounded, slightly sinuous rib; the linear ribs are found in the very young shell, but have not been detected in the older forms of this variety. In all of these varieties the septum, whieh is very characteristie of the species, continues the same. The figure of the septum given in the first volume, is from a weathered specimen, and has lost all of its more minute details. Besides the parts figured and deseribed, the ventral lobe seems to he very large, and projects a spur beyond the suture, the points reaching to and intruding between the tips of the lower braneh of the inferior lateral.

\section{A. Temamaensis, Gabb.}

(A. subtricarinatus, Gabb (not d'Orb.); Pal. Cal., Vol. 1, p. 60, pl. 10, fig. 4.)

Further study of this shell leads me to believe that it is distinct from subtricarinatus. It differs from that species in having three distinet, acute dorsal lieels; 
d'Orbigny's shell possessing but onc in the middle, the undulated dorsal ends of the lateral ribs making the other two. The Californian speeics bears a scrics of ribs on the sides, all larger in proportion than in the Frencli shell, and entirc, while in the other they are alternately bifurcate; that is to say, the ribs are altcrnately an entirc one, reaching from the umbilical to the dorsal margin, and a $Y$ sluapcd rib dividing on the middle of the shell, and scnding two branches to the dorsum. 'In sizc, and in proportionate size of volutions, the two shclls are closely allied, as well as in general style of ornament; and so far as we yet know, the geological horizon of the Californian is very ncar that of the European specics. The absolute age of the Cretaccous strata of the northern part of the State has not yet becn fully determined; but, as will be explained in the proper place, it scems not improbable that representatives of both the Gault and Lower Green Sand will be found included in what has here been provisionally tcrmed the Shasta Group. It is apparently from this member that the present speeies is derived.

\section{A. Suctaensis, Meek.}

Pl. 21, Fig. 11, $a, b$.

(A. complexus var. Suciaensis, Meek ; Proc. Phil. Acad., 1861, p. 317.)

A very characteristic, but not very perfeet specimen of this species was found near Monte Diablo. Mr. Meek, to whom I sent sketches, says that he considers it identical with the Sueia Island form; differing in some of the details of the septum, but not sufficiently to indieate a specific differenee.

A. JUGALIS, n. s.

Pl. 22, Fig. 12, $a, b, 13 a$.

Shell small, rounded discoidal, whorls rapidly increasing in size and deeply embracing; body whorl sub-appressed on the sides or, in some cases, regularly arched, dorsum rounded; umbilicus small, deep, margin rounded, or sub-angulated. Surface polished, or marked only by a few faint, sinuous lines, arching forwards, and more distinct on the dorsum; besides these there is a variable number of thickened ribs in the substance of the shell, starting from the umbilical margin, and curving outwards and forwards sinuously, and crossing the dorsum, where they are most strongly marked; these ribs, from three to six in number on a single volution, are more prominent on the internal than on 
the external face of the shell, and leave corresponding grooves on the cast.

Septum composed of a dorsal, suprerior and inferior lateral, and four supplementary lobes. Dorsal lobe long, bifurcate for twofifths of its length, with two small points over the siphuncle; the branches bearing two spurs on the outer side; above the branches are one large and two smaller spurs. Dorsal saddle deeply divided into three irregular branehes. Superior lateral lobe pretty symmetrically trifureate; the upper lateral braneh being a little more complex than the lower; two spurs above on each side. Lateral saddle like the dorsal, but a little smaller. Inferior lateral lobe much smaller than the superior, slender, unsymmetrical, obliquely bifurcate near its end, and bearing a large branch on the upper side near the middle. Supplementary saddles bifurcate; supplementary lobes rapidly diminishing in size to the umbilical angle, and resembling in fundamental structure, the inferior lateral.

Diameters : greater, 1.6 inelı ; lesser, 1.25 inclı; diameter of umbilieus, .2 ineh; height of aperture from umbilieal angle, .9 ineh; width, .65 ineh. $\mathbf{A}$ speeimen from Benicia gives for the greater diameter, 2. ineh; lesser, 1.5 ineh; umbilieus, .28 inch; height of aperture as above, 1 . ineh; width, 1 . inch.

This shell, though one of the most ineonspieuous, is nevertheless one of the most interesting of the California Ammonites, being the only member of the family known to occur at the same time in the Martinez and Tejon Groups. It is found at Martinez and Benieia in the former group, and I have colleeted it in the latter, in place, at the very top of the series at the eoal-mines near Clayton. Plate 10, figs. 5 , and 68 , Cal. Peport, Pal., Vol. 1, are from a speeimen from above the eoalbeds on Coal Mine Hill, near Mount Diablo.

\section{A. Whitneyt, h. s.}

Pl. 22, Fig. 14, $a, b$.

SHELL discoidal, whorls about six, rounded, slightly embracing, increasing gradually in size; sides rounded, slightly converging towards the dorsum; which is regularly arched; umbilical margin abruptly rounded; umbilicus broad, shallow. Aperture sub- 
ovate, considerably wider towards the ventral margin tnan near the dorsal, but slightly emarginated by the encroachment of the preceding volution. Surface marked by simple, straight, rounded ribs, arising on the umbilical margin, and continuing over the dorsum.

Septum composed of a dorsal, a superior and an inferior lateral, and two supplementary lobes to the umbilical margin. Dorsal lobe divided for half its length, deeply notched over the siphuncle, each branch tridentate on the end, carrying one compound and two simple spurs on the outer side; body of the lobe bearing two large spurs and one smaller one above. Dorsal saddle broad and divided into two compound bifurcatc branches. Superior lateral lobe as long as the dorsal, narrow and bifurcate in the middle, each branch being trifurcate on the end, and carrying one large spur above on the onter side; body of the lobe with two or three smaller spurs above. Lateral saddle like the dorsal, but a little more simple. Inferior lateral lobe shorter than the superior, divided a little below the middle into one complex branch on the ventral side, and two more simple oncs towards the dorsal; above this division is a large transverse spur on each side. Inferior lateral saddle small and deeply bifurcatc. First supplementary lobe small, bifurcate, the lower branch tridentate; the remaining lobe consists of but three small spurs.

From the Shasta Group, Cottonwood Creek, Shasta County.

Diameter, 4.25 inch; diameter of umbilicus, 1.6 inch; height of aperture from top of inclosed volution, 1.8 inch; greatest width, 1.8 inch.

\section{A. Stoliczkanus, n. s.}

Pl. 28, Fig. 16, $a$.

SHell thick, robust, heavily costate; whorls five or six, increasing rapidly in size, enveloping nearly one-half of the preceding volution; sides arched, most prominent in the middle, or a little nearer the umbilical than the dorsal margin; back broadly rounded, nearly flat; umbilicus large and deep, the umbilical 
face of the volutions nearly straight, the margin rounded. Surface ornamented by large ribs arising on the margin of the um. bilicus, and crossing the dorsum transversely; these ribs all show a tendency to tuberculation, though alternate ones only carry the tubercles well developed; these are arranged as follows: one small one on the umbilical angle, a larger one, slightly flattened in the direction of the rib, and placed on the middle of the side of the whorl; and a very large one, strongly flattened in the same manner, and placed on the dorsal face between the median line and the dorso-lateral margin; the arrangement of the last pair of tubercles is such that the dorsal face of the shell appears to carry a deep and broad median groove. The alternating, intermediate ribs in some cases are tuberculate, and reach the margin of the umbilicus, while in others, they are nearly obsolete, and occasionally entirely wanting.

The septum seems to be composed of a dorsal and two or three lateral lobes, quite small but complex, and with large saddles.

Diameter, 3.7 ineh; diameter of umbilicus, 1.4 ineh; height of aperture, 1.4 ineh; width, 1.8 ineh.

This is one of the most strongly eharaeterized speeies of the California Cretaeeuus, having no near allies in shape or ornamentation in the State, so far as known. It belongs to the same group as $A$. tubcrculatus, Sby., A. Michelinianus, d'Orb., and A. Archiacianus, d'Orb., three speeies eharaeteristie of the Gault of Europe. The first of these has a deep, narrow groove on the dorsum, and large tubereles, but no transverse ribs; the seeond is eharaeterized by similar tubereles, but in the plaee of single ribs proeeding from the umbilieus aeross the dorsum, the ribs bifureate on tbe umbilieal margin, and on the dorsal free the tubereles nre eonneeted by a series of oblique ribs, presenting a zigzag appearanee. The nearer ally is $A$. Archiacianus; but while this resembles it in both the transverse seetion and in the general arrangement of the tubereles, it has no ribs on either the sides or baek, the spaees between the series of tubereles being, in a measure, ehannelled.

I take pleasure in dedieating it to my friend Dr. Ferd. Stoliezka, Palæontologist of the Geologieal Survey of India, in reeognition of his able and valuable labors among the Cretaeeons fossils of that eountry.

From the Shasta Group, Cottonwood Creek, Shasta County. A single speeimen obtained by Mr. Mathewson. 


\section{A. FRATERNUS, n. s.}

Pl. 23, Fig. 15, $a, b$.

SheLL small, whorls rounded, deeply enveloping; sides rounded and eonverging towards the dorsum, whieh is regularly convex; umbilieus deep, moderate in size; umbilical margin rounded. Surface marked by slightly sinuous ribs, nearly straight, arising on the umbilical margin, and eontinuing over the dorsum; every fourth or fifth of these ribs is a little larger than the others, and bears a prominent flattened tuberele on the border of the umbilicus. An occasional eonstriction exists between the ribs.

Septum composed of a broad dorsal, a large superior lateral, a very small inferior lateral, and two supplementary lobes. Dorsal divided for about a third of its length, earrying two large serrate teeth over the siphunele, each braneh with a large, and two smaller spurs on the outer side; body of the lobe with three large spurs above the division. Dorsal saddle bifureate, eaeh braneh subdivided. Superior lateral lobe trifureate, the branehes nearly equal and trifid at the extremities; a large and a small spur above the fork. Lateral saddle nearly as wide as the dorsal. Inferior lateral lobe not more than half as long as the superior, trifid on the end, with two small spurs on the upper side and one on the lower. Supplementary lobes very small and trifid.

Diameter, 1.4 inch; diameter of umbilicus, .45 inch; height of apcrture, .55 inch; width, .6 inch.

A single specimen, nearly a miniature of $A$. Traskii, in general appearance, but with almost identically the septum of $A$. Hoffmannii, differing only from that of the latter species in some of the details. It cannot be the young of either of these species however, because though its surface resembles Traskii the septum is radically different; while all of its cxternal details, ihe form and ornamentation, arc entircly unlike any of the very large number of Hoffmannii that I have cxamined. No spccimen of that species that $I$ have ever scen shows the slightest trace of tuberculation, and in none are the ribs so marked. The single constriction on the surface of the specimen before mc, is not due to a thickening of the shell-substance, but is as strongly marked on the outside of the shell itself as on the cast. Another point, though of less importance than the above, is that this PAL. VOL. II. -19 
shcll is from the Martinez Group, while both of the other specics are peculiar to the Shasta Group.

Locality: Martinez Group, Benicia ; found by my lamented friend, Mr. Rémond.

\section{TURRILITES, Lam.}

T. Oregonensis, Gabb.

(T.sp. indet., Gabb; Pal. Cal., Vol. 1, p. 73, pl. 20, fig. 201.)

At the above place I indicated the cxistence of the first known spccies of the genus on the West Coast, but did not feel warranted in naming it from so small a fragment. I have since seen several fragmentary specimens from Oregon, the fincst of which is from the Cretaccous deposit on the Crooked Creek of the Des Chutes, the cxistence of which was made known by the Geologieal Survey in 1864 . The shell is very constant in its seulpture, the spire elevated, and the whorls increase slowly in size. The longest spiral I have seen is between two and three inches in length.

\section{ANCYLOCERAS, d'Orb.}

\section{A. Rémondi, Gabb.}

Pl. 23, Fig. 17.

(Crioceras (Ancyloceras ?) Rémondi, Gabb; Pal. Cal., Vol. 1, p. 75, pl. 14, fig. 24,24a.) (Ancyloceras sp.?, Gabb; loc. cit., p. 78, pl. 15, fig. 30.)

An examination of the septum of an undoubted fragment of the spiral part of this species, proves beyond question that the opinions I cxpressed in regard to the straight portion were correct. The speeies will therefore have to stand under the above name.

\section{A. percostatus, Gabb.}

Pl. 24, Fig. 19.

(Crioceras percostatus, Gabb ; Pal. Cal., Vol. 1, p. 77, pl. 16, 17, fig. 26.)

This magnificent species was described from fragments, and in the specimen figured, the outer portion fitted so neatly over the inner volution, that I was misled into bclieving that they were parts of a continuous spiral. Additional material 
has thrown light on the subjeet, and proves that the elosely eostate porions figured on Pl. 16, belong together as the spiral, sueeeded by a long subquadrate arm without ribs, the "outer whorl" with the large ribs being a part of the reeurved portion of a true Ancyloceras. The speeies is enormous, but variable in size. The speeimen figured was about thirty inehes in length, while one very perfeet speeimen in the cabinet of Mr. Voy, now deposited in the museum of the College of California, is but seventeen inehes long.

\section{? A. Lineatus, n. s. \\ Pl. 23, Fig. 18, $a, b, c$.}

This species is only known by two fragments of the straight limb. The section is ovate, a little narrower on the ventral than on the dorsal side; surface marked by numerous, very slightly oblique linear ribs, with broad concave interspaces; each rib bears on its dorso-lateral face a minute tubercle.

Septum composed of a dorsal and ventral lobe, and two laterals on each side, giving three saddles on each side. Dorsal lobe divided a little below the middle, with two teeth over the siphuncle, each branch bearing two spurs on the outer side, one large spur and one very small one on the body of the lobe. Dorsal saddle broad, deeply divided into two bifureate parts. Superior lateral lobe broad, as long as the dorsal, bifurcate in the middle, each branch again bifurcate, and with a large spur on its outer side above the subdivision; the body of the lobe bears a single large spur above the fork. Lateral saddle like the dorsal. Inferior lateral lobe like the superior lateral, but a little smaller. Ventral saddlc like the others, but a little smaller. Ventral lobe shorter than the others, trifurcate, the branches dentate, two small spurs above the branches.

This septum is unlike any other in California, unless it be that of? Hamites quadratus, which it resembles in the number and fundamental plan of structure of the lobes. In that species the saddles are proportionately very much larger, and the lobcs are more compact; the degree of complexity could be accounted for by the difference in size. The two shells differ materially in form. 
Length of the largest fragment, 2.7 inch; greater diameter, .85 ineh; lesser, .7 inch; distance between the ribs, .1 inch.

One specimen from the Shasta Group (?) Cottonwood Creek, another from near Folsom, from "Rock Corral," found by Mr. Gorham Blake.

\section{HELICANCYLUS, N. Gen.}

General form resembling Ancyloceras; shell commencing with a dextral, open, descending spiral, as in Helicocercs, the spiral afterwards opening, the shell becoming straight for a definite distance, and then recurving as in Ancyloceras.

The rclations of this genus are obvious; it is an Ancyloceras in which the spiral portion, instead of forming in the same plane, descends as in Helicoceras.

It may be questioned, how far these aberrations, from the regular spiral form of the more typical Ammonitide, may retain a generic value. It is by no means difficult to take the leading genera Ammonites, Turrilites, Baculites, \&c., and find trenchant characters; but it becomes more difficult to find sharp lines of division when we take all the species of the family and cndeavor to place each in a welldefined genus. Successive discoveries are every year introducing new links into the chain of gencra, until it seems almost impossible to imagine any $n$ əw form, or any combination of characters on which new genera can be founded.

In this aberrant group of Crioceras, Helicoceras, Ancyloceras, Heteroceras, Anisoceras, Tuxoccras, and Helicancylus, it seems inevitable that the addition of any other form must almost unavoidably result in the destruction, or coalescing of some of the at present received genera.

The present genus is founded on a single species, of which I am so fortunate as to possess an almost entire series of fragments of all the parts. Although allied to Pictet's Anisoceras, it differs in the young shell being a rcgular descending spiral, and not sinuous. That author figures, in his "Traité de Palcontologie," a very crooked fragment, such as could by no possibility belong to the present shell, any more than it could be a part of a Helicoceras or a Turrilite.

The figures of various species of Anisoceras, in Pal. Suisse, show a style of ornament very similar to the present one.

In his description, M. Pictet says: "Il paraît charactérisé par une forme plus irrégulière que chez ancune autre céphalopode. La coquille dans le jeune âge est sinueuse, formant une spirale irrégulière héliciforme, à tours disjoints, ayant tous une double courbure, et ne pouvant pas être compris dans un plan. Plus tard elle se redresse ct s'infléchit en crosse comme les Ancyloceras." It will be seen that the above definition cannot apply to the present genus. 
The same author anticipated the occurrence of this genus, as will be seen by consulting the analytical table of the group in Part 3, Palcontologie Suissc. He defines the thrce allicd gencra thus:

"Une spire composée de tours disjoints, ct une crosse. Ancyloceras."

"Portion spiralc très courtc et irrégulière, a tours très-écartés, une très-grande crosse comprise dans unc plan. Anisoceras."

"Spire régulière d'Ilelicoceras terminée par une crosse. Cas possible non encore obscriê."

Therc is one claracter, to which attention has been called, both by this author and by Dr. Stoliczka, in Palmontologia Indica, which, if found to be constant in Anisoceras, will serve as a good means of distinguishing the two genera; I fear, howercr, that a generalization on this point may yet be prematurc. I refer to the style of the septum. Pictet defines his genus as possessing six lobes and six saddles, the two laterals being bifurcated and nearly equal. Stoliczka more properly reduces the number to five, and speaks of the ventral as being rather a lobule than a distinct ventral lobe, on account of its small size and simple ornament in all the species yet examined. The present spccies has six lobes, the ventral being normally large, and the lateral being deeply trifurcate, the inferior latcral not more than half the length of the superior; thus resembling much more the style of Ancyloceras than Anisoceras.

Among the spccies referred to Anisoceras, and yet but imperfectly known, there are some that seem open to tlie suspicion of belonging in the present group. The most marked case is perhaps Hamites tenuisulcatus, Forbes, Ancyloceras id., d'Orb., Anisoceras id., Stol. The figure of the spiral portion in Pal. Ind., Vol. 3, pl. 85, fig. 14, certainly looks like our genus, and there seems to be nothing incompatible with this view, in the figure by Forbes in the Geol. Transactions. Its septum is as yet unknown.

\section{H. AQQuicostatus, Gabb.}

Pl. 25, Fig. $20 a-g$.

(Ptychoceras aquicostatus, Gabb; Pal. Cal., Vol. 1, p. 74, pl. 13, fig. 20.)

SHELL rather small; spiral portion with the spire low, the height being about equal to twice the thickness of the outer volution; whorls increasing very gradually in size, nearly in contact; number of whorls unknown, but from the rate of increase in diameter, about four or five (one and a half preserved); the straight limb was apparently about twice as long as the diameter of the spiral; the larger terminal limb seems to 
have inereased more rapidly in diameter than the others, is not perfectly straight, but bends a little outwards, giving a convex line longitudinally on its ventral face. Surfaee of the spiral portion ornamented by oblique, nodulated ribs; these are small, linear, and arched forwards on the ventral faee, large on the sides and dorsum, and more advanced on the lower than upper side, being oblique on the dorsum; they earry three pairs of nodes, one on each side of the median dorsal line, one on eaeh dorso-lateral margin, and one on the middle of eaeh side. The straight limb retains these ribs, which here lose their obliquity, but retain all the other characters; in addition, on this part of the shell, between each pair of these ribs, is placed a smaller, simple rib. On the return limb, the ribs lose all their tubercles, beeome even in size, aente, and with coneave interspaees; the section of this portion of the shell is rounded subquadrate, a little narrower on the dorsal than on the ventral side.

Septum eomposed of a dorsal and ventral lobe, and two laterals on each side. Dorsal lobe broad, divided for half its length, eaeh branch carrying three equal spurs on the outer side; above this, on the body of the lobe, are one large and three smaller spurs. Dorsal saddle divided into tivo bifureate branehes. Superior lateral lobe longer than the dorsal, broad, and earrying three slender, nearly equal branches; the middle one trifid on the end, the upper lateral mueh more complex than the lower; above these, on the body of the lobe, are two or three spurs, diminishing regularly upwards. Lateral saddle like the dorsal, but a little smaller, and obliquely divided. Inferior lateral lobe not more than half as long as the superior, slender and equally trifurcate. Ventral saddle like the others, but smaller. Ventral lobe nearly as long as the dorsal, slender, trifurcate, eaeh braneh trifid, the laterals bearing two spurs above on the outer side; the body of the lobe earries two spurs above the origin of the branehes.

Total length, 6 inches; diameter of spiral, 2 inches; diameter of volution at termination of spiral, .6 inch; height of apex, .9 inch; length of longest fragment of first straight limb, 4. inches; length of longest fragment of return limb, 2.7 
inches; greater diameter of aperture of same, $1.3 \mathrm{inch}$; lesser diameter, 1.1.5 inch. It is very probable that these fragments do not all belong to the same individual. The specimen figured in Vol. 1, Pal. Cal., seems to be more nearly in proportion to the spiral herein described. That fragment shows the recurve, and on one or two of the ribs of the thinnest part, can be seen tubercles corresponding in character to those described above.

\section{DIPTYCHOCERAS, N. Gen.}

\section{Three straight limbs in contact, partly enveloping.}

This genus bears the same relation to Ptychoceras that Hamites does to Hamulina. It is a Ptychoceras in every respect, execpt that it has an additional limb which incurvcs, enveloping both the preceding to a slight degree only.

Stoliczka and d'Orbigny both notice the occurrence of species of Ptychoceras with more than two limbs, and the former author amends the definition of the genus so as to cover the case. His description is as follows: "Elongated, lanceolate, straight, once, twice (or many times?) reflected; the siphuncle dorsal, the sutures divided into six lobes and six saddles, all of which (excepting the ventral lobe?) are bipartite." With all deference to so high an authority as Dr. Stoliczka, I am not prepared to accept this definition of Ptychoceras, unless, at the same time, we modify the characters of sevcral other genera of the family. If the number of straight limbs is not of gencric value, then why not include Baculites also? In the present state of our knowledge, it seems that there is a well-defined group of species characterized by two straight, parallel limbs, the larger, or newer of which never develops beyond a certain point on the length of the smaller; while another group, including P. Dupinianum, d'Orb., P. Forbesianum, Stol., and the following species, has this larger limb continued and again reflcted. Should it be aseertained that there is a gradation between these two, or that other speeies have more than two reflections, then there will be good grounds for doubting the validity of mY genus; until then, I believe we have sufficient reasons for maintaining it.

Solcnoceras, Conrad, founded on a single specimen of Hamites annulifer, Morton, is a shell that had at least two straight limbs, one partially enveloping the other; the greatcr of these has at its large end, a little offset or deflection, apparently indicating an abrupt bending backwards, or outwards, of the shell, and not an incurving, as in the present form. Whether this was the case, what was the entire form of Solenoceras, or whether it can be at all separated from Ptychoceras, are as yet unknown. All the information we possess on the subject is obtained from a unique specimen, which shows no peculiar generic characters, and which was, in plain truth, separated from Ptychoceras only on suspieion. 


\section{LeVIS, n. s. \\ Pl. 25, Fig. 21, $a, b$.}

SHeLt moderate in size, limbs long and slender, section of the smaller limb sub-elliptical, slightly flattened on the ventral side; second limb subcircular, very slightly emarginate by the encroachment of the preceding branch; last limb suddenly incurved, and extending to (or beyond?) the middle of the shell. Surface plain, polished, and ornamented only by a few extremely faint undulations; the outer or final limb, which is in places very much broken, had one or more constrictions or internal ribs, which do not scem to have been visible on the external surface; one or two such internal ribs seem to have existed, though in a much less degree, on the middle limb. Septum composed of a dorsal, a veutral, superior lateral, and two supplementary lateral lobes; dorsal lobe deeply divided into two curved branches, each branch bearing one very large spur on the outer side; the body of the lobe with one lateral spur above on each side. Dorsal saddle broad, divided into two branches, that on the upper side being again divided. Superior lateral lobe shorter than the dorsal, more robust, and resembling it in pattern. Lateral saddle like the dorsal, but smaller, and both branches simple. First supplementary lobe very oblique, small and trifurcate. Second straight and simple. Ventral lobe longer than the dorsal, slender, trifurcate, the middle branch toothed, the laterals trifid; above these branches, one large spur on each side.

Length of speeimen (less a little of the smaller end), 2.65 ineh; total length, 3. ineh?; length of small limb retained in the speeimen, 1.4 inch $(=1.75$ inels total ?) ; length of larger limb, $1.6 \mathrm{inch}$; (aperture partly destroyed); approximato diameter of aperture, .45 inch; diameter in middle of middle limb, .35 ineh; diameter in middle of small limb, 12 ineh.

From the Shasta Groun, Cottonwood Creek, Shasta County. Not rare; diseovered by Mr. Mathewson.

The present speeies secms very elosely allied to Pty. Forbesianum, Stol.; Pal. Ind., Vol. 3, p. 196, pl. 90, fig. 11, but is twiee the size of that shell, and differs in the 
presence of the faint undulated ribs on the larger limbs, and in the presence of the well-marked internal ribs. Unfortunately no septum was detected in the Indian species, and we cannot be absolutely certain of their specific difference, since the characters on which the two forms are separated, are among those which are most subject to variation.

\title{
BACULITES, Lam.
}

B. occidentalis, Meek.

(B. ovatus? Meek (not Say), Trans. Albany Inst., Vol. 4, p. 48.)

(B. occidentalis, Meek; loc. cit., p. 49.)

(B. sp. indet., Gabb ; Pal. Cal., Vol. 1, p. 81, pl. 17, fig. 28, 28 a; pl. 14, fig. 28 b.)

As yet, only known from Vancouver Island, whence Mr. Meek obtained specimens, which he referred doubtfully to Say's species, poirting out some differences and suggesting the above name, should thesc differences prove constant. More recently in his enumeration of the Crctaceous fossils, in the Proceedings of the Philadelphia Academy, and in the Smithsonian Check List, he has elevated the form to the rank of a distinct species, in which $I$ follow him.

\section{GASTER OPODA.}

\author{
FUSUS, Lam.
}

F. TUMIDUs, n. s,

Pl. 26, Fig. 22,

SHELL elongated fusiform, thick; spire elevated, whorls six or seven, sides swollen, outline sinuous; body whorl concavely sloping above, tumid in the middle, narrowed in advance. Aperture broad above, narrow below, canal produced; outer lip simple (?), inner lip slightly incrusted. Surface marked by about ten short broad longitudinal ribs or undulations on the middle of the whorl, crossed by numerous revolving linear ribs.

PAT. VOL. II. -20 
Length, 1.15 inch + ; width, .6 inch. The total length of a perfect specinen would probably be about 1.4 inch.

Very rare. A single specimen from the Martinez Group, at Martinez; Coll. by Mr. Mathewson.

This shell is allicd to $F$. Martinez, but differs in the more narrowly tumid character of the whorls, and the fewer longitudinal ribs. F. Martinez, so far as known, is characteristic of the Tejon Group.

F. OCCIDENTALIS, n. s.

Pl. 26, Fig. 23.

SneLL small, robustly fusiform; spire moderately elevated, turriculated; whorls five, angulated, sloping straight above, body whorl slightly convex below. Aperture broad, angulated posteriorly, produced in a narrow canal in front; outer lip simple, inner lip incrusted. Surface marked by about thirteen or fourteen small nodes on the angle of the whorls, and by numerous revolving ribs, about six or seven above the angle, and from sixteen to twenty below, two or three of which are larger than the others.

Length of broken specimen, .6 inch; total length, about .7 inch; width of body whorl, .45 inch; width of aperture, .2 inch. With the preceding.

NEPTUNEA, Bolt.

S. G. Tritonofusus, Beck.

(Atractus, Ag. preoc.)

N. (T.) Cretacea, n. s.

Pl. 26, Fig. 24.

SHeLl thin, short, rounded fusiform; spire low, whorls five, rounded. Aperture broad, angulated behind, produced into a curved canal anteriorly; outer lip simple, thin, columella curved, excavated in the middle. Surface smooth, or if sculptured, carrying only very minute markings. 
Length, $1.7 \mathrm{inch}$; width, 1 . inch ; length of aperture, $1.2 \mathrm{inch}$.

The only specimen I have seen is from Martinez, from the Tejon Group, or from the beds intermediate betwcen that and the Martinez Group, wcst of the town. It is so weathered that the sculpture, if any, is obliterated. The only shell with which this could be confounded, even in casts, is Fasciolaria lacviuscula, and from that it can be readily distinguished by its more gibbous form and curved canal.

\section{N. MUCRONATA, n. s.}

Pl. 26, Fig. 25.

SHELL moderate in size, thin, rounded fusiform; spire elevated, acute; whorls seven, rounded; suture sharply defined, linear; body whorl regularly convex, swollen in the middle, excavated in advance. Aperture large, acute behind, continued into a (slightly curved?) canal in advance; outer lip simple, thin; inner lip slightly incrusted; canal (from lines of growth) moderately produced and a little deflected. Surface marked by a few faint striæ of growth, and by regular, small, revolving impressed lines.

Length, $1.1 \mathrm{inch}$; width of body whorl, .6 inch; length of aperture, $.65 \mathrm{inch}$. Not common in the Martinez Group, at Martinez; Mathewson. The shell resembles somewhat Fusus Californicus, but can be distinguished by its more slender acuminate spire, the entirc absence of longitudinal ornament, thinner shell, more convex form, the curved canal, and by the details of sculpture. $F$. Californicus has not, as yet, been found at so low a horizon, stratigraphically, as the present species.

\section{PALAATRACTUS, N. Gen.}

I propose this name for a group of Fusoids of more or less pyriform shape, with a low spire, thick shell, slightly twisted columella, simple outer lip, inner lip incrusted, and with a heavily ribbed or cancellate surface. They cannot with propriety be placed in any of the heretofore described genera of Fusinc; Neptunec has a more or less elevated spire, and its sub-genus, Tritonofusus, with a low spire, is very thin, and the surface smooth, or marked only by fine lines; Cantharus has the outer lip crenu- 
lated or striate internally; Tritonidea has a tooth on the posterior part of the columellar lip, and the other genera are obviously untenable.

\section{P. CRASSUS, n. s.}

Pl. 26, Fig. 26.

SHELl small, pyriform, heavy; spire low, whorls five, rounded; suture impressed. Surface marked by prominent revolving ribs, crossed by irregular longitudinal ribs or lines; these latter are variable in size, number, and disposition, generally having a more or less regular arrangement, being of about the same size as the revolving ribs, and producing nodes or tubercles on the latter where they cross; between the larger markings are very numerous fine lines of growth. Aperture broad in the middle, acute behind; outer lip simple, inner lip incrusted; canal moderate and slightly twisted.

Length, .62 inch; width, .45 inch; length of aperture, .5 inch.

From the Shasta Group, from a cañon in the foot-hills, a mile south of the road from Colusa to the Sulphur Springs, near the eastern margin of the Coast Range, Colusa County. Rare.

\section{ERIPACHYA, N. Gen.}

SHELl short, robust, subovate to subfusiform, spire moderately elevated. Aperture broad, terminating in advance in a very short canal or a mere notch; outer lip simple; inner lip more or less heavily incrusted. Surface marked by longitudinal ribs and revolving lines.

The present genus is proposed to receive three species described in Pal. Cal., Vol. 1, under the name of Neptunea, but which possess a series of characters in common, sufficiently different from the typical species of that genus, to warrant their separation. They are small shells, about an inch in length, robust, thick, and want entirely the recurved canal of Neptunea; and they cannot, with any greater propriety, be placed in any of the other described genera of the family. They are 
all peculiar to the older divisions of our California Cretaceous, and two out of the three species arc represented by but few specimens, and are confined to single localities.

E. ponderosa, Gabb.

(Neptunea ponderosa, Gabb; Pal. Cal., Vol. 1, p. 88, pl. 18, fig. 38.)

The most common of the three species, numerous specimens having been obtained at Tuscan, or Lick Springs, by Dr. Veatch. It has also been found at Pence's Ranch, Butte County.

E. perforata, Gabb.

(Neptunea perforata, Gabb ; Pal. Cal., Vol. 1, p. 89, pl. 18, fig. 39.)

Referred by error to pagc 81 on the index to the plate.

Not rare at Cottonwood Creek, Shasta Group; unknown elsewhcre.

A small shell, half an inch long.

\section{E. Hoffmannit, Gabb.}

(Neptunea Hoffmannii, Gabb ; Pal. Cal., Vol. 1, p. 90, pl. 18, fig. 41.)

Found with the preceding. Rare.

\section{? Neptunea gracilis, Gabb.}

This shell, and $2 N$. supraplicata, were referred to Neptunea, because they agree with that genus, more than with any other. They however possess some characters which render this reference doubtful, and more perfect material than has yet been obtained will perhaps warrant their separation.

\section{PERISSOLAX, Gabb.}

\section{P. Blaker, Con. Sp.}

(Busycon? Blakei, Con.; P. R. R. Rep., Vol. 5, p. 322, pl. 2, fig. 13.)

(P. Blakei, Gabb ; Pal. Cal., Vol. 1, p. 92, pl. 21, fig. 110.)

A specimen before me, of this shell, from Martinez, has three distinct revolving angles on the body whorl, instead of the two on the specimen figured as above, and the spire is not so high. Mr. Conrad denies my determination of the species, saying that his Busycon Blakei is very different. I cannot agree with him, and am perfectly satisfied that this is the shell described by him from Tejon, where it is very abundant, and where no similar shell has ever been found. I would be less 
positive were it not that he holds the samc opinion about several other species, and it is not probable that a single boulder should have contained several species, never afterwards encountered by Professor Brewer on lis visit, by me on the three or four oceasions when I have bcen there, or by Dr. Horn, who collected there assiduously for a number of months!

Mr. Conrad, in the Smithsonian Check List, mentions a P. Gabbii, Con. No such species has ever been deseribed; can this be the shell? Mr. C. says he does not remember, and $I$ have no other clue.

\section{SURCULA, H. \& A. Ad.}

S. preattenuata, n. s.

Pl. 26, Fig. 27.

Shell very long and slender; spire unusually high; whorls angulated, numerous (perhaps ten or morè). Surface marked by a row of small nodes on the angle of the whorls, and by numerous small revolving ribs, crossed by irregular lines of growth, the latter, at times, elevated, so as to form imperfect longitudinal ribs. Aperture short, as compared with the height of the spire; outer lip broadly and shallowly emarginate above the angle of the whorl, produced and regularly convex below.

Length, 2.5 inch (about); length of apcrture, 1 inch; length of body whorl, .7 inch.

From the Tejon Group, San Diego; Dr. Cooper.

This species ean be at once distinguished by its extremely elongate form, and the comparatively short aperturc. $\Lambda$ s is usually the case with very slender fossil shells, it is nearly impossible to obtain an entire specimen, and I havc never been able to see the wiole spire. From the rate of diminution in size of the whorls towards the apex, I believe the species to have had at least ten, and perhaps onc or two more volutions.

\section{S. (Surculites) sindata, Gabb.}

P1. 26, Fig. 28.

(Conus sinuatus, Gabb; Pal. Cal., Vol. 1, p. 123, pl. 29, fig. 227.)

SheLl broadly fusiform, spire shorter than the aperture, turreted; whorls about six to six and a half, angulated, concave 
above, the upper volutions undulated or minutely nodose on the angle; suture channelled. Aperture moderately wide, biangular posteriorly, outer lip deeply notched above the angle, prominently rounded in the middle. Surface marked by lines of growth, and by a few faint revolving lines anteriorly.

Length, 1.9 inch ; width, .9 inch; length of apcrture, 1.2 inch.

From the Tejon Group, Tejon; at Cañada de las Uvas and Arroyo de los Alisos.

The species was originally deseribed from a single broken specimen, which since proves to have been immature. It showed none of the characteristic portion of the lower part of the mouth, and while I noted the peculiar curve in the outline of the body whorl, I was misled into believing it to be a Cone. Having since obtained better specimens, I am fortunately able to refer it to its proper genus. It belongs to a group in the genus that has been indicated by Mr. Conrad, under the sub-generic name of Surculites, and which is characterized by the angulated form of the present species. Mr. Conrad's type is an Eocene fossil.

\section{S. (SURCDLITES) INCONSPICUA, n. s.}

Pl. 26, Fig. 29.

SHeLr small, spire high; whorls six (?), angulated, concave above and on the side; body whorl broad, rapidly tapering in advance. Surface marked by two large revolving ribs, one on the angle and one a little in advance; between these, and on the upper part of the whorl, the surface is covered with minute revolving lines; below the lower of the two ribs, the remainder of the shell is marked by small revolving ribs. Aperture broad, biangular posteriorly; outer lip notched above, produced below; inner lip slightly incrusted; canal moderate.

Length of fragment, .47 inch; total length of shell, about.55 inch; width of body whorl, .3 inch ; length of aperture, .25-.28? inch.

From the Martinez Group, Martinez. Rare; Mathewson.

\section{HETEROTERMA, N. Gen.}

Shell short, robust-fusiform, spire low, body whorl dilated, canal moderately long, straight; outer lip broadly notched or emarginate near the suture; columellar lip incrusted. 
The general form, and the notch at the upper part of the outer lip, place this genus, without question, in the Pleurotomida, but its thick shell, low spire, and very broad body volution, separate it unmistakably from all the known genera of the family.

H. TROCHOIDEA, n. s.

Pl. 26, Fig. 30, $30 a$.

Shell depressed fusiform, spire low, whorls five and a halt, concave above, body whorl bicarinate, each carina bearing a row of large rounded tubercles; suture linear, undulated, the upper margin of each whorl being attached to the upper row of tubercles on the preceding volution; between the two caring on the body whorl, the surface is obliquely concave; anteriorly it is deeply excavated and produced in a long straight canal. Aperture broad above, narrow and straight below; outer lip simple, broadly emarginate on the upper surface, inner lip incrusted. Surface ornamented, besides the tubercles, by minute revolving lines, smaller above than below, and showing a tendency in advance, to alternation in size.

Length, restored from lines of growth, .9 inch; width of body volution, .6 inch; length of aperture, .65 inch.

Rare in the Martinez Group, Martinez; Mathewson.

\section{BELA, Leach.}

B. CLATHRATA, n. s.

PI. 26, Fig. 31.

Shell small, subfusiform; spire elevated, whorls six, the first

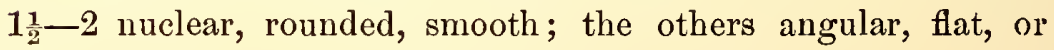
slightly concave on the sides, and sloping above a little concave to plane; body whorl excavated below, bicarinate in the middle. Aperture moderately broad, tapering slightly in advance; outer lip simple, broadly excavated above, and slightly prominent in the middle; inner lip slightly incrusted. Surface marked by two 
revolving angles, between which, and above to the suture, are very minute revolving striæ; in advance of the lower angle, these striæ are larger and alternate in size; besides these the whole surface is covered with longitudinal raised lines corresponding to the striæ of growth.

Lengtl, . .37 inch; width of body whorl, .18 inch; length of apcrture, .2 inch. A single specimen from the Tejon Group, Martincz; Mathewson.

\section{CORDIERA, Rouault.}

\section{MitRAFORMIS, n. s.}

Pl. 26, Fig. 32.

Shels elongate, mitræform, spire comparatively low; number of whorls unknown; body whorl concave, angulated and undulated above, long, slender, and sinuous below. Aperture long and narrow, biangular posteriorly, narrowing gradually in advance; outer lip thin, obliquely emarginate between the angle and the suture, very slightly sinuous, nearly straight below; inner lip rather heavily incrusted, and bearing two (possibly three) oblique folds near the middle; canal produced, straight; suture impressed, linear, slightly irregular. Surface bearing ten or eleven prominent, slightly oblique longitudinal ribs, starting from the angle of the whorl, and becoming obsolete before reaching the middle; crossing these are numerous revolving ribs, small above the angle and large and rounded below.

Length (less the greater part of the spire), .9 inch; length of aperture, .65 inch; width of body whorl, .4 inch.

This beautiful little shell has no near relatives in form, either in the genus, or among its associates in the formation, in other genera. Its discovery is of especial interest, since it is only the second known Cretaceous spccies of the genus, the other being also Californian; and this bcing by far the oldest species yet discovcred. The existence of its congener in the Tejon Group was one of the numerous arguments used by an eminent authority, in support of his view of the Tertiary age of that formation; while we now have the genus carried back to near, if not to the PAL. VOL. II. -21 
very base of the Cretaceous. So much for a priori reasoning, based on negative grounds.

From the Shasta Group, Colusa County, near the Hot Sulphur Springs.

\section{TRITONIUM, Link.}

T. Californicum, il. s.

Pl. 26, Fig. 33.

SHell small, thin; spire elevated, whorls seven and a half, the first three and a half minute, nuelear, rounded and smooth; the others rounded and eostate; suture impressed, variees about two to a volution, prominent. Aperture wide in the middle, aeute posteriorly, and warrowed and produeed into a short eaual anteriorly; outer lip aeute on the margin, bearing a thiekened, rounded varix behind; inner lip slightly inerusted; eanal abruptly defleeted, moderate in length. Surfaee ornamented by about twenty aeute longitudinal ribs, and about a dozen revolving ribs; between eaeh pair of the latter are two or three fine elevated striæ; where the first four of the larger revolving lines eross the longitudinal ribs, at each point of intersection, is a minute tuberele.

Length, .7 inch; length of aperture, .45 inch; width of body whorl, 4 inch.

Rare in the Tejon Group, Tejon. Dr. Horn.

\section{S. Gen. TRACHYTRITON, Meek. \\ T. (T.) Tejonensis, n. s.}

Pl. 26, Fig. 34.

Shelt large, fusiform, thin, spire elevated; whorls four and a half or five, subangulated, nodose. Aperture broad, anterior end?; outer lip (? dentate or) plieate within, inner lip straight anteriorly; body whorl flattened and sloping above, bearing about ten flattened tubereles on the angle, and with two or three larger and numerous smaller revolving lines anteriorly, 
Length of a broken specimen ( 2 cast), 2 inch; width of body whorl, 1.3 inch ; length of aperture, 1 inch + .

I have secn but a single specimen of this species, and that a cast, but I venture to describe it because it is so diffcrent from all the other fusiform shclls in the formation, that it cannot well be mistaken. The impression on the matrix, and traces of ribs on the cast, together with small fragments of shell, show the surface to hare been costate; and some plications at the oral margin of the cast, indicatc either a dentate or plicate labrum. Therc is a trace of but a single varix, and that high up on the spire; this shows traees of numerous, well marked teeth.

Tejon Group, Arroyo de los Alisos, Tejon.

T. (T.) FUSIFORMIS, n. s.

(Pal. Cal., Vol. 1, pl. 18, fig. 45.)

Shell small, short fusiform, spire nearly as long as aperture; whorls five and a half, angulated. Aperture moderately wide, biangular posteriorly, narrowing in advance; inner lip slightly curved and incrusted; upper portion of body whorl flattened. Surface marked by fifteen or sixteen longitudinal ribs, diminishing from the angle towards the suture, and extending anteriorly to near the middle of the shell; these are crossed by numerous revolving ribs; on the external surface can be seen one or two arrests in growth, indicated by a crowding and increase in size of lines of growth, and corresponding to a more strongly marked internal varix.

Figure, above quoted, somewhat enlarged; onc of the varices is shown on the body whorl, near the aperture. Tejon Group, Tejon. Rare.

This shell was overlooked while writing the descriptions for the first volume of the California Report; and I did not detect the mistakc until too late to rectify it.

\section{BRACHYSPHINGUS, $N$. Gen.}

Shell bucciniform, short, robust, thick; spire low; aperture large, notched anteriorly; outer lip simple; inner lip incrusted with a smooth callus; surface longitudinally ribbed or striate.

The nearest allied genus to this, is Cominella; they differ, in the present form wanting the high spire, and the compression of the whorls near the suture, char- 
acteristie of that genus; it also resembles some of the forms of Bullia, especially in the sub-genus Buccinanops, but the form of the aperture will at onee distinguish them.

\section{B. Liratus, Gabb.}

(Buccinum liratum, Gabb; Pal. Cal., Vol. 1, p. 96, pl. 28, fig. 211.)

\section{B. SinUatus, n. s.}

Pl. 26, Fig. 35.

Shell short, thick, subovate; spire low, whorls four to four and a half, almost entirely hidden, except on their upper margin; suture variable, sometimes deep and bordered by a thickening of the succeeding whorl, sometimes almost linear; body whorl swollen in the middle, and marked by sinuous longitudinal lines of growth, and in some specimens by undulations of the same form; on the anterior part of the shell are a few minute revolving lincs. Aperture broad in the middle, acutc behind, narrowed in front, and dceply and narrowly notched; a rib revolves backwards from this noteh around the anterior portion of the shell, occupying the former position of the notch at the successive stages of growth; outer lip simple, slightly sinuous in outline; inner lip heavily incrusted.

Length, 1.4 ineh; width of body whorl, .9 ineh; length of aperture, 1.1 ineh. A very variable shell, both in the height of the spire and in the intensity of the surfaee markings. Some speeimens are perfectly smooth exeept for the revolving lines, while others are strongly eostate by well marked but irregular sinuous longitudinal ribs produced by irregular growth.

From the Tejon Group, Martinez; found over a small area, but apparently common, where they do oeeur; all the speeimens were obtained by Mr. Mathewson.

\section{BULLIA, Gray.}

\section{Molopophorus, N. Sub-Gen.}

Short, robust, spire moderately elevated, suture bordered by a more or less distinct carina. Surface longitudinally ribbed or 
striate. Aperture obtuse behind, and very slightly notehed; outer lip simple, inner lip very slightly inerusted, sinuous; anterior notel small but distinetly defined.

The present form has the carinated, or subangulated posterior portion of the whorl, characteristic of some species of the genus Bullia, but more nearly the shape of the sub-genus Buccinanops, d'Orb.

The species is of the same general style as Bullia (Buccinanops) moniliferum, Val., but its very low spire and small anterior notch, separate it.

B. (M.) STRIATA, n. s.

Pl. 26, Fig. 36.

SHeLl small, eompact; spire moderately elevated, whorls five, eonvex, the first two smooth, the others longitudinally ribbed; suture deep, undulated, and bordered by a rib on the top of the suceeeding whorl. Surface ornamented by irregular longitudinal striæ, some of which are more elevated than the others, and form slightly sinuous ribs. Aperture broad, outer lip simple, very obscurely notehed adjoining the suture; inner lip very sinuous, slightly incrusted; anterior noteh deep, narrow and oblique, bordered by two faint, variable folds, whieh revolve obliquely around the eanal.

Length, .3 inch; width, .2 inch; length of aperture, .21 inch.

Rare in the Tejon Group. Dr. Horn.

A pretty littlc Buccinoid shell, entircly unlike anything else in California.

\section{TURBINELLA, Lam.}

T. CRASSITESTA, n. s.

Pl. 26, Fig. 37.

Shell moderate in size, subfusiform, thiek, spire low; whorls four to four and a half, suture distinet. Surface marked by four or five large revolving ribs on the middle and upper part of the whorl, and smaller ones anteriorly, the larger of these are crossed 
by twelve to fourteen broad longitudinal ribs, each point of intersection being marked by an enlargement of the revolving ribs. Aperture broad in the middle, narrowed and straight in advance; outer lip acute and undulated on the margin, inner lip thickened, flattened, and bearing two distinct oblique folds in the middle; canal produced, straight.

Length, 1.2 inch; length of aperture, 1 inch; width of bods whorl, .6 inch. Rare in the Martinez Group, Martincz. Mr. Mathewson.

\section{MITRA, Lam.}

M. cretacea, Gabb.

(Mitra cretacea, Gabb ; Pal. Cal., Vol. 1, p. 103, pl. 28, fig. 215.)

Since the publieation of the first volume of the Report, another specimen of this rare spccics has been obtained at the same locality, from the Tejon Group. It differs in some respects from the first, showing faint traces of crenulation on the angle of the upper whorls, the body whorl is more robust, and the anterior end, which was absent in the first specimen, is regularly tapering, making the apcrture slightly longer than the spire. All of the whorls are more distinctly carinated on the angle than in the specimen figured.

\section{FICOPSIS, Con.}

Mr. Conrad proposed this name to include a group of thin subfusiform shells, apparcntly allied to Ficus, but with a somewhat higher spire, and a straight, or nearly straight, and somewhat produced canal. The group seems to be a well defined one, and includes numerous species in the newer Cretaceous, and in the Eocene. Ifis type is his Ficus penitus, = Pyrula cancellata, Lea, of the Alabama Eocene.

The following Californian specics belong to this typc:

\section{F. Rémondi, Gabb.}

(Fusus (Hemifusus) Rémondii, Gabb ; Pal. Cal., Vol. 1, p. 87, pl. 18, fig. 36.)

F. Hornir, Gabb. (F. (H.) Hornii, Gabb ; Pal. Cal., Vol. 1, p. 86, pl. 28, fig. 206.) 


\author{
F. COOPERII, Gabb. \\ (F. (H.) Cooperii, Gabb; Pal. Cal., Vol. 1, p. 86, pl. 28, fig. 207.)
}

\title{
UROSYCA, N. Gen.
}

SHeLL thin, pyriform, spire very slightly elevated, body whorl long. Surface transversely striate or cancellate. Aperture broad above, narrow and produced below; outer lip simple, inner lip very slightly incrusted; canal long, slender, and slightly twisted.

This genus seems to be closely allied to Ficopsis, but differs from it in its more pyriform shape, and in the canal bcing more slender, proportionally longer, and contorted instead of being perfectly straight. Mr. Conrad has placed Ficopsis as the next genus to Ficus, and I think correctly. We have thus, at least three gencra of this group, characterized by their general ficoid shape, thin shells, low spires, and more or less delicate sculpture. In the absence of the animals, it is difficult to ascertain the family relations of shells so closely allied in form as these are to some of the Fusince, and it is not improbable that there arc many fossil shells called by the promiscuous appellations of Fusus and Pyrula, which really belong to this family.

\section{U. CAUDATA, n. s.}

Pl. 27, Fig. 38 .

ShELL moderately large, pyriform, spire low, whorls five, the upper rounded, the body whorl subangulated by three nearly equidistant, nodose, revolving carinæ; top of whorl slightly sloping, interspaces between the ribs slightly concave, anterior portion deeply excavated. Surface crossed by numerous small revolving striæ. Aperture broad, outer lip simple, inner lip slightly incrusted, canal long, slender, slightly twisted.

Length, 2.35 inch; length of aperture, 2.15 inch; width of body whorl, 1.3 inch.

Rarc in the Martinez Group, Martinez. Mr. Mathewson.

Resembles somewhat Ficopsis Cooperii in stylc of ornament, but can be distinguished by its greater size, lower spirc, broader body whorl, the presence of three instead of two carinæ, and by the curved canal. 


\section{SYCODES, N. Gen.}

SHELL subpyriform, nearly of the shape of an immature Cyproca; spire low. Aperture simple, narrowing slightly in advance, and with a shallow anterior notch; columella straight in front. Surface plain, or ornamented only by fine lines.

I described the species on which this genus is founded, in the first vol. Pal. Cal., under the generic name of Ficus. Sinee then I have been able to determine that the form is constant, and, as $I$ then supposed, the specimens are adult. So far as I know, it is the oldest known form allied to Ficus, being from the Chico Group. The species is rare, all the specimens coming from a single locality.

The genus is evidently elosely related to Ficus, differing principally in its proportionally thicker shell, in the straight, short columella, and the anterior part of the mouth eontinuing wide to the extremity, instead of narrowing as in all the allied genera.

S. CYPREOIDES, Gabb.

(2 Ficus cyprceoides, Gabb ; Pal. Cal., Vol. 1, p. 58, pl. 19, fig. 105.)

\section{EUSPIRA, Ag.}

An Oolitic genus, possessing all of the leading characteristics of the following shell, the flattened and angulated upper surface of the whorls, the closed umbilicus, and slight thickening of the columellar lip. In all of its peculiarities, except the flattening of the upper portion of the volutions, it seems closely allied to Ampullina and Lunatia.

\section{E. alveata, Con. sp.}

(Natica alveata, Con.; P. R. R. Rep., Vol. 5, p. 321, pl. 2, fig. 8.)

(Amauropsis alveata, Gabb; Pal. Cal., Vol. 1, p. 110, pl. 19, fig. 59 ; pl. 21, fig. 111.)

(Ampullina alveata, Con.; Smithsonian Check List, No. 459.)

Characteristic of the Martinez and Tejon Groups. Common. 


\title{
NEVERITA, Risso.
}

\author{
N. GLOBOSA, n. s.
}

P1. 27, Fig. 39.

Shell subglobose, spire low, acute; whorls rounded, five and a half, rapidly increasing in size, the last sloping above, and slightly flattened on the side; umbilicus small but well marked. Aperture suboval, narrower above than below; outer lip simple, inner lip with a heavy callus, prominent above, slightly flattened on the inner face, the portion over the umbilicus flat, rather thin, and sometimes bearing a faint transverse groove.

Length, .75 inch; width, .72 inch; length of aperture, .68 inch; width of aperture, .32 inch.

From Neverita secta, its nearest ally, this shell ean be distinguished by its much more globose form, the upper part of the whorl being round instead of sloping almost regularly from the apex; it is not so wide at the base; the callus is very large posteriorly, instead of being thin and narrow as in secta, and the portion over the umbilicus is small, rounded and flat, instead of being, as in secta, large, convex, and triangular.

From the Tejon Group, ten miles west of Griswold's, on the road from San Juan to New Idria, and southeast of the "Sheep Well.".

\section{AMPULLINA.}

\section{A. striata, n. s.}

Pl. 27, Fig. 40.

SHELL small, compact, subglobose, whorls five, rounded, suture deep, whorls in some cases rounded, subtruncated above, adjoining the suture, in other specimens regularly rounded. Surface ornamented by numerous, minute, revolving, impressed lines, more or less interrupted by the lines of growth. Aperture subovate, widest in advance; outer lip thin, simple; inner lip simple, terminated anteriorly by a narrow, flattened margin commencing in the umbilical region; umbilicus closed or minutely perforated. 
Dimensions of a small specimen : Length, .68 inch; width of body whorl, .57 inch; length of aperture, .52 inch.

From the Martinez Group, Martinez; Mr. Mathewson.

A variable shell, the greatest variation being in the shape of the body whorl, some specimens being much more expanded and oblique than others.

\section{TEREBRA, Brug.}

T. Californica, n. s.

Pl. 27, Fig. 41.

Shell small, slender, elongated, sides flattened; whorls eleven, flattened on the sides, the body whorl slightly convex a little below the middlc, and excavated below. Surface ornamented by small, slender, and slightly sinuous longitudinal ribs, the upper margin of the whorl bordered by a slightly thickened rim. Aperture elongate, outer lip simple, inner lip incrusted, canal a little deflected and twisted, notch very small.

Length, .52 inch; width of body whorl, .08 inch; length of aperture, .11 inch. From the Tejon Group, Martinez; a single specimen found by Mr. Mathewson.

\section{CHEMNITZIA, d'Orb.}

C. Planulata, Gabb.

(C. Spillmanni, Gabb (not.Con.); Pal. Cal., Vol. 1, p. 115, pl. 19, fig. 70.)

On examining Mr. Conrad's original specimen, I am inclined to agree with him that there are some good points of specific difference, and therefore propose the above name.

\section{PUGNELLUS, Con.}

\section{P. hamulus, Gabb.}

Pl. 27, Fig. 42, $42 a$.

(P. hamulus, Gabb ; Pal. Cal., Vol. 1, p. 81, pl. 20, fig. 124 ; pl. 18, fig. 48.)

This shell is extremely variable from the changes of age. The two figures quoted above give a young shell, and one that has acquired its lip and incrustation. 
In the latter, pl. 20 , fig. 124, there is shown an angular notch on the outer lip, also mentioned in the description as one of the specific characters. The shcll is a common one, and the discovery of numerous specimens shows that this notch is by no means constant. A very old, but small individual before me, is so heavily incrusted that its original form is entircly lost, and several expert conchologists have been, on first sight, at a loss to recognize at all, even its family relations. It shows no trace of a spire or of volutions, and the outcr lip is almost as large as the body whorl, while the canal is very short, and incurves at almost a right angle to the axis of the shell. Were I less familiar with the species, I should hesitate in calling this specimen by the same specific name, but $I$ have seen so many variations in its form, that $I$ have no doubts whatever on the subject.

\title{
S. Gen. Gymnarus, Gabb.
}

P. (G.) Manubriatus, Gabb.

(Pugnellus manubriatus, Gabb; Pal. Cal., Vol. 1, p. 125, pl. 29, fig. 229, a.)

(Pugnellus (Gymnarus) manubriatus, Gabb; Amer. Jour. Conch., 1868, p. 139, pl. 13, fig. 4, 5.)

In Palæontologia Indica, Gasteropoda, p. 21, Stoliczka suggests that this species may be identical with $P$. contortus, Sby. sp., which it closely resembles in sculpture. It differs in being a much more slender shell, the callosity never heavy, the outer lip more regular in form, and the anterior canal straight, instead of being incurved as in contortus. In the Journal of Conchology, I recently suggested a subgeneric separation, mainly on the peculiar form of the canal. I have examined a large series of specimens, and cannot agrec with Dr. Stoliczka, that the thinness of the callosity is due to injury, since the same characters obtain in all the individuals. Nor is the canal broken in the specimen figured. I have never detected the slightest indication of a curve in this portion of the shell, where the specimens were perfect, or nearly so.

\author{
CYPRAEA, Linn. \\ S. Gen. Luponia, Gray. \\ C. (L.) Bayerquer, Gabb. \\ Pl. 27, Fig. 43, $a, b, c$. \\ (? Cypraea Bayerquei, Gabb; Pal. Cal., Vol. 1, p. 129.) \\ Shell small, ovoid, convex, broadest near the upper end, \\ gradually tapering convexly anteriorly; spire entirely hidden even
}


in the young shell; base sub-flattened. Aperture narrow, outer lip considerably produced posteriorly, thickened and strongly incurved, slightly carinated near the extreme anterior end, both lips bearing numerous small teeth, placed deep in the mouth, and not continued downwards to the margins. Surface smooth, or marked by indistinct longitudinal lines of growth. The young shell is thin, the lines of growth pretty distinct, the aperture broadly expanded in the middle, and apex is submerged.

A perfect specimen has been obtained in the Tejon Group at Martinez, and Dr. Horn sent me two immature ones from Tejon.

\section{S. Gen. Epona, H. \& A. Ad. \\ C. (E.) Mathewsoni, n. s. \\ Pl. 27, Fig. 44, $a, b$.}

Shell small, thick, very convex, inequilateral, most convex in the middle and a little above; the outer lip straighter than the opposite side; ends but slightly produced, anterior end more than the posterior; basc convex. Aperture linear, outer lip produced behind more than the inner, thickened by a callus, expanded laterally, especially towards the ends, where the margin is thinner and subacute; inner lip regularly incrusted, carinated in advance; both lips bearing a few large teeth, those on the inner lip being continued as raised lines, to a considerable distance on the facc of the body whorl. Surface smooth, the limits of the thickening of the outer lip being pretty well defined.

Length, .43 inch; width, .28 inch; height, .22 inch.

This beautiful little shell has no relations in the formation. It approaches most nearly the sub-genus Epona of $\mathrm{H}$. and A. Adams, but differs from the more typical species of that group, in the very small degree to which the ends are produced. I have never seen but a single specimen, which I obtained from Mr. Mathewson, who found it in the Tejon Group at Martinez. 


\section{ANCHURA, Con.}

A. Falciformis, Gabb.

(Aporrhais falciformis, Gabb ; Pal. Cal., Vol. 1, p. 127, pl. 20, fig. 83.)

Subsequent discoveries show that the canal of this shell is very long and straight. From being cxtremely slender, it is rarely preserved. One specimen from Chico Creek has an aperture, including the canal, as long as the spire. All of the species described in the above work as Aporrhais, belong to the genus Anchura.

Following the custom of nearly all writers on the subject, I have there and elsewhere, heretofore quoted Aporrhais as a genus of Petiver, Dacosta, \&c. Recently having had my attention specially called to the subject, I investigated the history of the generic name anew for myself, and have published the result of my study in the American Journal of Conchology, 1868, page 143. Neither Aldrovandi, Petiver, nor Dacosta, ever knew the name as connected with the true Aporrhais. They all applied it to various species of Pterocera; a good opportunity for some conchological revolutionist to make another "restoration," and quote Pterocera as the genus Aporrhais of Aristotle, Aldrovandi, et id omne genus.

\section{A. transversa, n. s.}

Pl. 27, Fig. 45.

SHell small, fusiform, spire elevated; whorls rounded, their number unknown, suture deep. Surface of upper whorls, and upper part of body whorl, strongly cancellated by longitudinal and transverse ribs; body whorl strongly carinated near the lip, the carina running out, and forming a sort of midrib or keel to the lip. Lip long, transverse, curved upwards, the upper margin regularly concave, tip acute, lower margin broken; canal long, straight.

Length unknown; about .7 inch; width of body whorl, .2 inch; including length of lip, .5 inch.

From the Martinez Group, Martinez. 


\section{?. A. CARINIFERA, n. s.}

Pl. 28, Fig. 46.

I propose this name for a minute shell, of which I have but a single rather imperfect cast. It is short, robust fusiform, the whorls rounded, the body whorl tapering gradually in advance, instead of ending in a slcnder canal, as in the typical Anchuras. The lower whorls carry a single prominent rib on the middle, and the margin of the mouth is broken where (if it is an Anchura) the lip process should take its rise; the whole surface is ornamented by numerous revolving strix, visible both on the shell and, to a less degrce, on the cast, becoming strongly marked on the inner margin of the outer lip.

Length of the fragment, less two or three volutions of the apex, .32 ineh.

From the Martinez Group, Martinez.

I have referred this little shell to the genus Anchura, with eonsiderable hesitation, beeause I have no proof that it possessed the expanded lip, and beeause the anterior canal, whieh in all the known speeies is slender, and more or less produeed, is, in this ease, short and robust. Further speeimens will be neeessary before its true generie relations ean be definitely settled.

\section{HELICAULAX, Gabb.}

\section{BICARINATA, n. s.}

Pl. 27, Fig. 47.

SheLL robust fusiform, spire elevated; whorls six ; sides regularly sloping, body whorl bicarinate, the upper edge of the whorl joining the upper carina of the preceding volution, so as to produce a continuous slope to the apex. Surface marked by numerous, minute revolving lines, one or two of which, below the lower angle of the body whorl, are larger than the others; a series of small nodes on each carina of the last whorl. Aperture broad, continued posteriorly by a long, slender canal running up the spire to near the apex, and then curving outwards and backwards; 
outer lip expanded, biangular, bordered by a thickened margin, the posterior and lateral margins concave, a digitate process at the posterior angle; anterior angle (not produced?); inner lip expanded over the front of the body whorl, and continued up the spire as high as the canal, very much thickened and longitudinally striate on the outer margin; canal and anterior portions of the shell unknown.

Length, less all of the anterior canal, 1. inch; width of body whorl, .45 inch ; width, including outer lip, .9 inch.

From the Shasta Group, Cottonwood Creek, Shasta County'

A peculiar shell, easily distinguished even in fragments by the regular slope of the spire, the biangular form of the body whorl, the heavy incrustation of the inner lip, and the long, solid posterior canal. The genus, proposed by me in Amer. Jour. Conchology, 1868, page 145, is a strongly characterized member of the Aporrhaidce, and seems to be the nearest ally of Aporrhais in the Cretaceous, having many of the characters of that genus, and forming the link between it and Anchura; the lip being much more like the latter than the former genus, unless perfect specimens of the present species should prove, by being bi-digitate, that this is not a constant character.

\section{H. costata, n. s.}

Pl. 28, Fig. 48.

Shell small, broadly fusiform, spire elevated; whorls six or seven, angulated in the middle, sloping, and nearly straight above, slightly convex below; above the angle are numerous fine striæ, below are five or six subacute revolving ribs, with broad concave interspaces. Aperture broad above, narrowed in front, posterior canal well marked, and curving backwards; outer lip and anterior canal unkuown; inner lip thinly incrusted.

Length, less the anterior canal, .47 inch; width of body whorl, .3 inch.

From the Martinez Group, Martinez.

A little shell, strongly characterized by its revolving ribs, and very angular whorls. The posterior canal, in the specimen, reacles to the second whorl above the aperture, and seems to have been attached to the spire still further, beforc being deflected; the groove is unusually deep, and the specimen was probably not entirely mature, judging both from this character, and from the thinness of the inner lip, which, in all the other species, is heavily incrusted. 


\title{
LOXOTREMA, Gabb.
}

\author{
L. TURRITA, n. s.
}

Pl. 28, Fig. 49.

Shell elongated, turreted, spire elevated, nearly twice the length of the aperture; whorls about six to six and a balf, slightly convex on the sides, abruptly truncated and flat on the upper margin. Body whorl marked by eight or ten revolving lines on the anterior half, crossed by sinuous lines of growth; both sets of markings being very variable in distinctness in different specimens. Aperture obliquely subquadrate, bordered on the inner side by a raised lip, the top retreating upwards, and very obliquely backwards; outer lip thick above and below, very thin in the middle, and with a strongly sinuous margin, most prominent near the anterior end; inner lip thick, its margin somewhat raised above the suface of the body whorl; anterior extremity of aperture not notched, but produced, and slightly channelled.

Length, 1.65 inch; width of body whorl, .8 inch; length of aperture (internal measure), .6 inch; width, .45 inch.

Common in the Tejon Group, ten miles west of Griswold's, between San Juan and New Idria.

This very pcculiar shell is evidently closely allied to Struthiolaria, but differs in the shape of its volutions, the elcvated spire, and the retreating backwards of the posterior margin of the aperture. It has the characteristic lip-like anterior end of that genus, and its sinuous outer lip. A marked character is the extrente thinness of the middle of the whorl, and the equally unusual thickness of the walls both above and below this point, rendering it almost impossible to obtain a specimen in which the middle of the outer lip is not broken away.

\section{ATRESIUS, N. Gen.}

SHELL elongate, spire elevated; whorls rounded, aperture ovate, slightly produced in front, outer lip entire, thin; columella not incrusted, imperforate; surface marked by revolving ribs. 
A non-pearly shell, resembling Tuba, Lea, and Spironema, Meek, but differing from both in being entirely imperforate, showing no trace even, of an umbilieal depression. It is most probably a member of the Littorinida, and should be placed near Spironema.

\section{A. Liratus, n. s.}

Pl. 28, Fig. 50 .

Shell small, thin, elongate; spire elevated, number of whorls unknown, whorls regularly convex, suture linear. Surface marked by about fourteen or fifteen subacute revolving ribs with concave interspaces, the space between the upper rib and the suture twice as broad as those below, first rib minutely crenulated, the whole surface crossed by fine but distinct lines of growth. Aperture subovate, a little produced and subangular in front, outer lip simple, thin, and showing faint traces internally of the ribs on the surface, columella curved.

Length (broken), .43 ineh; total length, .55 inch ?; width of body whorl, .37 inch; length of aperture, .28 inch.

From the Shasta Group, Colusa County, southeast of the Hot Sulphur Springs.

\section{TURRITELLA, Lam.}

\section{T. Martinezensis, $n$. s.}

P1. 28, Fig. 51 .

Shell robust, apical angle broad; whorls twelve, angulated, flat to concave above and below the angle; body whorl with two angles, the lower less prominent than the upper; in the spire but one angle is visible, the upper edge of the whorl being applied to the lower angle of its predecessor. Surface marked by two or three strong lines on the upper face, one between the revolving angles, and sometimes one or two below; besides there are numerous fine revolving lines, all crossed by faint striæ of growth. Aperture broad, outer lip slightly sinuous, inner lip flattened and somewhat twisted.

PAL. VOL. II. -23 
Length, 2.3 inches; width of body whorl, $.95 \mathrm{inch}$; length of aperture, .55 inch. From beds intermediate between the Martinez and Tejon Groups, Martinez. Numcrous specimens werc obtained at a single locality by Mr. Mathewson.

This shell is entirely unlike any other Californian Cretaceous Turritella, and resembles in its style of ornament some of the Eastern Miocenc species. It can be ensily identificd by its prominent single anglc on each whorl of the spirc, and by its broadly expanding form, approaching in this character to $T$. robusta of the Chico Group.

\section{NERITA, Linn.}

S. Gen. Theliostylu, Mörch.

$$
\begin{gathered}
\text { N. (T.) triangulata, n. s. } \\
\text { Pl. 28, Fig. } 52,52 a .
\end{gathered}
$$

Sheld small, thick, semi-ovoid, spire flattened; whorls two or two and a half, rapidly increasing in size. Surface divided by three angles into four uearly equal revolving planes, each plane marked by four or five rounded ribs with equal interspaces. Mouth rounded subquadrate, outer lip subacute on the margin, very thick bchind, and internally toothed; inner lip broadly expanded, nearly straight on its internal edge, and bearing a few large teeth, its surface coarsely pustulated.

Length, .28 inch; width, .38 inch; height, .25 inch.

Not common in the Tejon Group at New Idria, where I found but two specimens. It has never been encountered elsewhere.

\section{CALLIOSTOMA, Swains. Zizyphinus, Gray. \\ C. radiatum, n. s.} Pl. 28, Fig. 53.

Shent minute, broadly conical; whorls four (or more), sides flat, sloping; suture well marked. Surface ornamented by fourteen or fifteen large radiating ribs, which produce an undulation 
of the upper margin of the whorl, and abut against a strongly marked revolving rib bordering the lower angle; these are crossed by six or seven smaller revolving elevated lines; under surface marked by numerous, minute, revolving striæ. Aperture subquadrate; inner lip straight, slightly oblique and thickened.

Length, .16 inch; width, .18 inch.

This species is known by but a single specimen from the Chico Group of Texas Flat, Placer County. It is not improbably a young shell, though it already possesses four volutions. It has so well-marked a scries of characters that $I$ have ventured to describe it, since it cannot be confounded with any known species.

\section{ATAPHRUS, N. Gen.}

Shell thick, flattened subglobose. Surface smooth. Aperture subovate, oblique; outer lip simple, inner lip thick, rounded, uniting with the outer lip by a regular curve; umbilicus entirely hidden.

The present genus seems to occupy an intermediate position between the recent genera Oxystele, Phil., and Photinula, H. and A. Adams. From the former it differs in having a rounded columella, instcad of flattened; while from the latter, it can be distinguished by its smooth surface and the junction between the labrum and labium being by a regular curve in front, instead of the columella terminating in a point near the external edge, as in Photinula.

A. crassus, n. s.

Pl. 28, Fig. 54.

Shell small, thick, flattened subglobose, spire low; whorls four, rounded, revolving a little obliquely; body whorl slightly flattened below. Surface marked only by lines of growth. Aperture oblique, subcircular, columellar lip thick and rounded; umbilical region covcred by a smooth callus, which merges insensibly into the general surface.

Length, .18 inch; width, .28 inch.

Rare in tho Martinez Group (?) at Martinez. Specimens were sent me by Mr. Mathewson, but not so labelled that I can determine thcir horizon, and they are so carefully trimmed out of the matrix that $I$ have no associated spccies to assist me. 


\section{MARGARITELLA, M. \& H. \\ M. Angulata, n. s.}

Pl. 28, Fig. 55.

Shell small, thin, highly polished, spire elevated; whorls four and a half, convexly flattened on the sides, more or less truncated above; body whorl very slightly convex on the base. Surface bearing one, two, or three strongly impressed lines near the suture, crossed by oblique impressed lines forming variable series of crenulations; these oblique lines rarely extend far down on the sides, but hold the same direction as the lines of growth, which can occasionally be traced their entire leugth; besides these, there are sometimes faint revolving lines visible over the entire surface, more constant on the base; umbilicus moderate, its margin cvenly crenulated, its internal surface marked by strong revolving and radiating lines. Aperture subquadrate, margins simple.

Length, .25 inch; width, .23 ineh.

From the Martinez, or Chico Group, Martinez. There is a small deposit of the latter group near Martinez, and judging from the lithologieal eharaeter of the specimens, they seem to have comc from it; they were sent me by Mr. Mathewson.

This speeies ean be distinguished from $M$. crenulata by its higher spire and narrower umbilieus; they approach each other in the erenulation of the top of the whorl and the umbilical margin.

ACM AA, Esch.

\section{? A. Tejonensis, n. s.}

Pl. 28, Fig. 56.

Suelu small, low, ends unequal; anterior end broad, sides converging posteriorly; posterior end narrow, rounded; apex subcentral, slightly curved forwards. Surface marked by a few concentric lines, and six or eight faint radiating undulations.

Length, . 35 ineh; greatest width, .25 ineh; height, .1 ineh. 
A small inconspicuous species from the Tejon Group, Tejon; Dr. Horn. It can be distinguislicd from? A. (Patella) Traskii, of the Chico Group, by its ovate instead of elliptical outline, and by the beak curving markedly in advance; the radiating ornaments are also much less marked than in the Northern shell.

\section{ACT ÆONINA, d'Orb.}

\section{A. PUPOIDES, Gabb.}

Pl. 28, Fig. 57.

(2 A. pupoides, Gabb ; Pal. Cal., Vol. 1, p. 67, pl. 19, fig. 113.)

This shell was described from a single specimen in which the anterior half was broken away. I have since been able to verify my determination of the genus, and to ascertain the remaining characters of the species. The apex is variable, the form figured being the most common; in some cases it is higher, in others more blunt. The aperture is small, narrowed behind, rounded in advance; outer lip acute, slightly sinuous; inner lip heavily incrusted in advance by a broad, nonplicate callus, which extends around the anterior margin of the mouth, becoming regularly smaller, and disappearing at the anterior end of the outer lip.

The figure is natural size.

From the Shasta Group, Cottonwood Creek.

\section{ACT EONELLA, d'Orb.}

\section{A. OVIFORMis, n. s.}

Pl. 28, Fig. 58.

Sheld elongate ovoid, sides nearly parallel in the middle; spire unknown, whorls five or more, nearly straight on the sides, tapering, rounded in advance. Aperture narrow, straight, a little wider in front, inner lip heavily incrusted in advance, and with two moderate folds. Surface plain.

Length (less the spire, broken away), 1.8 inch; width of body whorl, .85 inch. A single broken specimen from the Chico Group, (?) Cotton wood Creek, Shasta County ; easily distinguished by its slender form and subparallel sides. 


\section{LIOCIUM, $N$. Gen.}

Sinel elongate, spire high, apex dextral. Surface polished and ornamented by striæ or punctate markings. Aperture ovate, not emarginate anteriorly; outer lip slightly sill uous, and bordered by a thickened rim; inner lip incrusted and smooth, bearing no folds.

The thickened lip, incrusted columella, shell texture, and sculpture of the surface of this shell, point to an alliance with Cinulia (Avellana) and Ringinella; while its elcvated spire, entire anterior end of the aperture, and the absence of teeth or folds, sufficiently separate it from all known genera of the family.

\section{L. punctatum, n. s.}

Pl. 28, Fig. 59.

Shel minute, elongate, slender, spire elevated; whorls seven, very slightly convex, suture faint, extreme apex rather blunt. Surface highly polished, and marked anteriorly by a few faint revolving lines of punctations. Aperture elongate oval, acute posteriorly; outer lip slightly sinuous, bordered by a thickened rim, about twice as thick as the shell walls, and longitudinally striate: this thickening continues uninterruptedly around the anterior end of the mouth; inner lip incrusted, smooth.

Length, .18 inch; width of body whorl, .06 inch; length of aperture, .06 inch.

Rare in the Shasta Group, south of the road from Colusa to the Hot Sulphur Springs in the first range of Foot Hills, Colusa County, associated with Cordiera mitroformis, Atresius liratus, and the following species. It is a beautiful little shell, and can be at once recognized by its minute size, slender form, polished surface, and by the characteristic punctate sculpture.

\section{RINGINELLA, d'Orb.}

R. POLITA, n. s.

Pl. 28, Fig. 60 .

SHELl small, subovate, spire prominent; whorls six, rounded, body whorl slightly flattened on the sides, narrowing in advance, 
suture linear, impressed. Surface marked by a few, distant, impressed, revolving lines, variable in number. Aperture long, rounded in advance, inner lip bearing one large and one smaller and less prominent fold in advance.

Length, .4 inch; width of body whorl, .24 inch; length of aperture, .27 inch. Not rare, with the preceding species.

This shell can be distinguished from all previously described members of the family in California, by the simple style of its sculpture, consisting of simple impressed lines; R. (Cinulia) pinguis having elevated ribs, Ringicula varia having grooves crossed by minute elevated lines, and the others being too different in shape to permit of a confusion.

R. PINGUIS, Gabb.

(Cinulia pinguis, Gabb ; Pal. Cal., Vol. 1, p. 112, pl. 29, fig. 221.)

\title{
A C E P H A L A.
}

\section{MARTESIA, Leach.}

\author{
M. clausa, Gabb.
}

(M. clausa, Gabb; Pal. Cal., Vol. 1, p. 145, pl. 22, fig. 115.)

This species was described from the Chico Group. During the last two or three years several specimens have been obtained from the Martinez Group, at Martinez, and from the Tejon Group at Martinez, ten miles west of Griswold's and Tejon. In these more recent forms I can find no charactcrs different from the original type; and Mr. Tryon, whose great experience in this family renders his opinion of especial weight, agrees with me in believing them all to belong to the same species.

\section{SOLEN, Linn.}

\section{S. Gen. Hypogella, Gray.}

S. (H.) CUNEATUS, n. s.

Pl. 29, Fig. 61 .

SHELL small, narrowed anteriorly, wider behind; hinge margin straight; beaks anterior, subterminal; margin slightly excavated 
under the beaks; anterior end narrowly rounded; posterior end broadly convex; base slightly sloping, and nearly straight; surface faintly marked by lines of growth.

Length, 1.25 inch; width under the beaks, . 3 inch; width near the posterior end, .4 inch.

A rarc shell from the Martinez Group, Martinez.

It can be distinguished from the young of Solen parallelus, by its widening posteriorly, and by the prominent beaks. From S. (Hypogella) Diegoensis, it differs in all the details of form.

\section{S. (II.) Diegoensis, Gabb.}

(S. (Solena) Diegoensis, Gabb; Pal. Cal., Vol. 1, p. 213, pl. 32, fig. 280.)

(Plectosolen Diegoensis, Con.; Smithsonian Check List, No. 240.)

When I used Browne's name for this subgenus, I did it without knowing the date of its publication, having been misled by H. and A. Adams. Scientific research in a ncw country, away from large libraries, is attended with great difficulties, such as only those who have had the experience, can understand. As I have already intimated elsewhere, I strongly disapprove of the usc of preLinnæan names in science, believing that the only way to arrive at a settled nomenclature, is to fix some definite date, behind which we cannot go, as has been done by the British Association. Gray's name of Hypogella is the oldest that we can use according to this rule. The name Plectosolen, was proposed by Mr. Conrad, without a description; and he has since informed me that he has abandoned it, finding it to be founded on untenable characters

\section{CORBULA, Brug.}

\section{Hornir, Gabb.}

Pl. 29, Fig. 62, $a, b$.

(C. Hornii, Gabb ; Pal. Cal., Vol. 1, p. 149, pl. 29, fig. 128.

This species was described from a cast of a left valve and a broken right value, Subsequently Dr. Horn sent me four other specimens, which enable me to correct some points in the diagnosis. The shell is almost exactly equivalve, the anterior end not usually so produced as in the figure quoted above; the left valve is strongly angulated posteriorly, the right valve not so much so; the surface is marked by fine and pretty regular concentric lines. 


\section{ALEFORMIS, n. s.}

Pl. 29, Fig. 63.

Shell large, broadly rounded in advance, narrow, produced, and truncated behind; beaks about a third of the length from the anterior end, high; posterior cardinal margin nearly straight, bordered by a broad, deep groove, extending from the beaks to the posterior end; base prominently and broadly rounded in the middle, sinuous behind. Surface marked by small, regular, concentric ribs.

Figure, natural size.

From a bed.intermediate in age between the Martinez and Tejon Groups, near Lower Lake village, Lake County.

The largest of our Californian Corbulas, and characterized by its narrow produced posterior end.

\section{ANATINA, Lam.}

\section{A. Quadrata, n. s.}

Pl. 29, Fig. 64 .

SHELL moderate in size, subquadrate; beaks nearly central; length and width about as eight to five; anterior end gaping, truncated, and with the corners rounded; posterior end convexly subtruncated, or rounded; base nearly straight, being a very slight curve, most prominent in the middle; the cardinal margin is slightly excavated in advance of the beaks, and sloping and nearly straight posteriorly. Surface marked by a few lines of growth.

Length, 2. inches; width, 1.25 inch.

From the greenish rock on the north shore of Departure Bay, Nanaimo, Vancouver Island; associated with Pecten Traskii, Trigonia Evansana, and other specics characteristic of the Chico Group in California.

Of somewhat the style of $A$. lata, but longer and more regularly quadrate; from $A$. Tryoniana it can be distinguished by being shorter, the ends being more distinctly truncated, and by its wanting the angular ridge descending from the bcaks; from $A$. incequilateralis it can be known by the more central position of the beaks, and the less rounded ends.

PAL. VOL. II. -24 


\section{PHOLADOMYA, Sby.}

P. Oregonensts, n. $\mathrm{s}$.

Pl. 29, Fig. 65.

SHeLl small; beaks large, prominent, anterior; anterior end sloping outwards and downwards from the beaks, nearly straiglit above, narrowly rounded below; posterior end prominently rounded, a little the most prominent above the middle; cardinal margin nearly straight behind the beaks; base broadly and regularly convex. Surface marked by small but pretty regular coneentric lines, crossed by a few faint radiating ridges, three or four of which, on the middle and anterior end, are distinct, the others obsolete.

Length, 1.2 inch; width, 7 inch.

Rare in the Chico Group, near the Toll House at the summit of the Siskiyou Mountains, near the southern boundary of Oregon.

From $P$. nasuta, this shell can be distinguished by its flatter form, the absence of the anterior truncation, its regularly rounded posterior end, and its obsolete radiating ribs. There is no other species on the West Coast, with which it could be confounded. P. subelongata, Meek, from Vancouver Island, has many more ribs, and they are most marked on the posterior portions of the surface.

\section{PLEUROMYA, Agas.}

\section{P. PAPYRACEA, n. s.}

Pl. 29, Fig. 66.

Shell very thin, beaks prominent, and placed about' a third of the length from the anterior end; cardinal margin sloping slightly, and nearly straight posteriorly, anterior end excavated under the beaks, and abruptly truncated below, very slightly gaping; posterior end narrowly rounded, gaping more than the anterior; base broadly convex. Surface ornamented by irregular concentric ribs. Posterior muscular scar small; pallial sinus broad and deep. 
Length, 1.7 inch; width, 1.2 inch; thickncss, .75 inch.

From Cotton wood Creek, probably from the Shasta Group. A number of specimens were collected by Mr. Mathewson in 1865, from a dark-colored rock, in which he does not seem to have found any other fossil; and beyond the meagre label, "Cottonwood," I have no information.

\title{
ARCOMYA, Agas.
}

\section{A. undulata, n. s.}

Pl. 29, Fig. 67.

SheLL very inequilateral, beaks prominent, strongly incurved, and placed about a third of the length from the anterior end; cardinal line nearly straight; base slightly convex; ends rounded, subtruncated, and nearly equal; both ends slightly gaping. Surface unknown, surface of the cast marked by a few large, concentric undulations.

Length, 2.25 inches; width, 1.3 inch; transverse diameter, .9 inch.

A single specimen, from Indian Creek, Butte County, belonging to Mr. Voy, of Oakland, who kindly loaned it to me for description.

Easily distinguished by its peculiar arcæform shape, from any other known fossil of California.

\author{
HOMOMYA, Agas. \\ H. concentrica, Gabb. \\ (Panopcea id., Gabb; Pal. Cal., Vol. 1, p. 148, pl. 22, fig. 119.)
}

From the Chico Group, of Tuscan Springs, Cottonwood Creek, and Martinez.

\section{MACTRA, Linn.}

? M. TENUISSIMA, n. s.

Pl. 29, Fig. 68.

SHeLl small, extremely thin, subequilateral; beaks cenitral, small, pointed slightly in advance; anterior end broadly and regularly rounded; posterior end obliquely and convexly sub- 
truncated; base regularly convex. Surface marked by minute, concentric striæ of growth; an angulated ridge runs from the umbones to the posterior basal angle. Pallial sinus deep and narrow.

Length, 1.1 inch; width, .8 inch; depth of one valve, .15 inch

A single spccimen, from thc Martinez Group, Martinez.

I have had this specimen a number of years, and have heretofore hesitated to describe it, hoping to obtain others, which would settle its exact gencric relations. That it belongs to the Linnæan genus Mactra, and even to that genus as restricted by Lamarck, I have little doubt; but from the nature of the matrix, and the extreme tenuity of the shell, it is impossible to expose the hinge, and $I$ am therefore unable to assign it at present, with any degree of certainty, to its proper place among the numerous closely allied genera of the family.

\section{CYMBOPHORA, N. Gen.}

Pl. 29, Fig. 69.

I propose this genus for one of the most common fossils of the Californian Cretaceons, Mactra Ashburnerii. The hinge is composed of a rather heavy hinge-plate, bearing a cartilage-pit, not sunk into its substance, as in the others of the Mactridoe, but, as it were, built up on its surface; a small, delicate, spoon-shaped process, laid obliquely under the beaks, its base being on, or slightly above the level of the hiuge-plate; in the right valve the cardinal tooth is single, very delicate, and nearly at a right angle with the anterior wall of the cartilage-pit; in the left valve the tooth is V-shaped, entirely separated from the pit, very slender, and articulates between the tooth and the pit of the opposite side; the lateral teeth are large and comparatively very robust.

This genus seems to be most nearly allied to Trigonella, H. and A. Ad., as figured in Gencra of Recent Mollusca, but differs in the character of the cartilage-pit, in the laterals being more robust, and in the cardinal teeth of the left valve being more removed from the pit. 


\section{Ashburnerit, Gabb.}

(Mactra Ashburnerii, Gabb ; Pal. Cal., Vol. 1, p. 153, pl. 22, fig. 127.)

Very characteristic of the Chico Group, and extending as high up as the Tejon Group, being found at all of the typical localities of both of these, as well as of the included Martinez Group.

\section{ASAPHIS, Modeer.}

\section{A. multicostata, n. s.}

Pl. 29, Fig. 70.

SHELl small, elongate subquadrate, inequilateral; beaks nearly central; cardinal margin sloping towards both ends, concavely in advance, convexly behind; base broadly and regularly convex; anterior end rounded; posterior obsoletely and obliquely subtruncated, convex; a broad, rounded angle running from the beaks to the posterior basal margin. Surface marked by about sixty small radiating ribs with somewhat wider interspaces, and crossed by a few lines of growth. Muscular scars large; pallial line unknown; an internal thickening runs obliquely downwards and forwards from the beaks to the anterior muscular scar, forming a marked depression on the cast.

Length, .68 inch; width, .4 inch; height of single valve, .1 inch.

A single mould and an internal cast, from the Crooked Crcek of the Des Chutes River, Central Oregon, east of the Cascade Mountains; associated with Trigonia Evansana, Turrilites Oregonensis, and other species, eharacteristic of the Chico Group. Thesc speeimens, beyond a doubt of the samc age as the fossils near Jacksonville and on the Siskiyou Mountains, in Southern Oregon, were obtained by me in 1864, while in Eastern Oregon. The occurrence of the formation east of the Caseade rangc, as proved by these fossils, was published in January, 186 $\bar{i}$, by Professor Whitney, in the Proceedings of the California Academy of Natural Scienees, Vol. 3, page 309. In July, 1867, Professor Blake announced the same faet, as a new discovery, in the American Journal of Scienee, page 118. I merely call attention to the circumstance here, to forestall any diseussion on the claim to priority of discovery, that might otherwise arise in the future; profiting by our experience in similar cases in the past. 


\title{
TELLINA, Linn.
}

\section{'T. RÉmondir, Gabb.}

\author{
Pl. 29, Fig. 71.
}

(T. Rémondii, Gabb ; Pal. Cal., Vol. 1, p. 156, pl. 22, fig. 132.)

Sincc publishing the original description and figure of this species, other and better specimens have been obtained, and $I$ am enabled to illustrate a more perfeet and older individual than in the first instanee. A slight difference will be observed in the line running posteriorly from the beak in the two figures, and the concentric ribs also seem to vary in size.

The present specimen, from iny own cabinet, was obtained by Dr. Horn at Tejon.

\section{T. Hoffmanniana, Gabb.}

Pl. 30, Fig. 72.

(T. Hoffmanniana, Gabb ; Pal. Cal., Vol. 1, p. 156, pl. 22, fig. 133, 133 a.)

This rather variable species is extremely common in the Martinez Group at Martinez, and has been found in the Chico Group at Penee's Ranch, as well as in the Tejon Group at Griswold's. I have now a single speeimen from the latter group from Martinez, associated with Turritella Uvasana, and other eharacteristic speeies. The present figure illustrates a common, and one of the most marked forms of the Martinez Group, Martincz, from the same bed with Pugnellus hamulus.

\section{T. RQUALIS, n. s.}

Pl. 29, Fig. 73.

SHeLL moderate in size, nearly elliptical, thin, flattened; narrow in its young state, proportionally broader as it grows older; beaks very slightly in advance of the centre, small; cardinal margins slightly convex; base broadly and regularly curved; ends rounded, the anterior a little the narrowest. Surface marked by lines of growtl, most prominent near the extremities.

Figure, natural size.

Rare in the Martinez Group at Martinez.

From T. ooides it ean be distinguished by its central beaks and nearly equal ends; 
from $T$. Mathewsonii by its more symmetrieal form, the outline forming a nearly regular ellipse; and from $T$. Hornii by that speeies being very mueh longer in proportion to its width.

\section{T. UNDULIFERA, n. s.}

Pl. 30, Fig. 74.

SHELL moderately large, thin, slightly convex, very inequilateral, broadly rounded in advance, tapering behind; beaks twofifths of the length fiom the anterior end, slightly pointed in advance; cardinal margin sloping rapidly and with a slight convexity to the posterior end, which is narrowly rounded; base prominent, convex. Surface marked by concentric ribs, forming large undulations towards the beaks, and gradually diminishing towards the base.

Length, 1.65 ineh; width, 1.4 ineh; height of single valve, .2 ineh.

From a soft, gray sandstone, west of Martinez, probably belonging near the upper portion of the Martinez Group.

The shell is, in all eases, so softened by decomposition that I am unable, in any of the speeimens, to expose the hinge, and therefore refer the speeies to the above genus with some hesitation. Judging from the imperfeet impressions left on some internal easts, the hinge has been very delieate, and seems more like that of a Tellina than of any other genus, though the external form and general appearanee render this determination somewhat doubtful.

In outline the shell is not unlike $T$. coides, but the posterior end is narrower, the beaks are more prominent, the surfaee is more convex, and the style of ornament will serve at once to distinguish the two speeies.

\section{DONAX, Linn.}

D. LATUS, n. s.

Pl. 30, Fig. 75.

SHELL broad, triangular, thin, very inequilateral ; beaks high, placed about two-fifths of the length from the anterior end; sides sloping, nearly straight, a rounded angle running from the beaks to the posterior basal margin, leaving a narrow area parallel with 
the posterior side; base broadly and pretty regularly convex, joining the other two sides of the triangle by narrowly rounded angles. Internal basal margin minutely dentate, the dentations corresponding with the external ribs. Surface ornamented by numerous fine, regular, radiating ribs, flattened on top, and with the interspaces somewhat narrower than the ribs.

Length, 1.1 inch ; width, .8 inch; height of single valve, .18 inch.

Rare in the Tejon Group, ten miles west of Griswold's, southeast of the Sheep Well, on the road from San Juan to New Idria.

This shell is remarkable for its thinness, and as much of the hinge as I have been ablc to cxpose, shares the delicate character of the shell itself.

\section{VENUS, Linn.}

\section{V. aquilateralis, n. s.}

Pl. 30, Fig. 76.

Shell small, triangular, the three sides forming a nearly equilateral triangle, with eurved sides; beaks small, pointed forwards, and placed very slightly in advance of the middle; cardinal margin sloping rapidly and slightly convex; base broadly and not very prominently convex, rounding up in advance, and uniting by a short curve with the anterior end; the posterior basal angle is much more acute than the anterior; anterior margin slightly excavatcd under the beaks, nearly straight below. Surface marked by small concentric ribs. Hinge composed of short, robust teeth; muscular sears large, the anterior being the deepest and most strongly marked; pallial sinus shallow; internal margin plain.

Length, .85 inch; width, .75 inch; height of single valve, .2 inch.

From the Tejon Group, from San Diego.

The nearest ally of this spccies is V. lenticularis, G., from Benicia, but the two shells can be easily distinguished by the more marked triangular form, and smaller size of the present one, and by the absence of a lunule, or, if it does exist, by its being large and very faintly marked. In $V$. lenticularis the lunule is small but distinct. 


\section{MERETRIX, Lam.}

\section{? M. FRagilis, n. s.}

Pl. 30, Fig. 77.

SHell small, extremely thin, elongate; beaks placed about twofifths of the length from the anterior end, prominent and pointed in advance; anterior margin excavated under the beaks, broadly rounded below; posterior ends regularly but more narrowly rounded; cardinal margin sloping convexly; base convex, most prominent under the beaks. Surface marked by concentric striæ, in some cases as visible on the cast as externally. Pallial sinus deep, oblique.

Length, .8 inch; width, .65 inch; depth of single valve, .15 inch.

Rare in the Martinez Group at Martinez, associated with Meekia sella, Pugnellus hamulus, \&c.

Allied in form to $M$. arata of the Chico Group, but differs in the details of outline, in its marked thinness, and in the surface ornament.

\section{HoRnII, Gabb. -}

Pl. 30, Fig. 78.

(Meretrix Hornii, Gabb ; Pal. Cal., Vol. 1, p. 164, pl. 23, fig. 144.)

As I stated in a note to the description of this species (loc. cit.) the figure in the first volume was not good. I now avail myself of the opportunity of correcting the error by the publication of a more accurate illustration.

\section{CARYATIS, Roem.}

In a monograph of the Linnæan genus Venus, Dr. Roemer adopts Cytherea, Lam., as a sub-genus, and proposes a "section" Caryatis, to receive a large group of species characterized by a more or less trigonal form; a shallow or superficial lunule, bordered by an impressed line; a narrow, subelongate, immersed ligament; a triangular pallial sinus with an obtuse apex; a thin, smooth, internal margin; and with a hinge composed of three divergent cardinal teeth, the anterior thin and direct, the posterior large and oblique, and with a remote postcrior lateral. These

PAL. VOL. II. -25 
charaeters are certainly of suffieient value to separate the group as a distinct genus, which, in addition to the living speeies, comprises numerous fossils in the Eocene, and some in the Cretaceous.

C. NITIDA, Gabb.

Pl. 30, Fig. 79.

(Meretrix nitida, Gabb; Pal. Cal., Vol. 1, p. 165, pl. 23, fig. 145, 146.)

A fine shell, very variable in form in different stages of growth, and eharaeteristie of the Chieo Group. The present figure is from a young shell, from Martinez. The largest specimen $I$ have seen is nearly two inehes in length.

\title{
THETIS, Sby.
}

\author{
? T. Elongata, n. s. \\ Pl. 30, Fig. 80, $80 a$.
}

Sheld small, very thin, subquadrate, gibbous, elongate; beaks large, incurved, a fourth of the length from the anterior end; cardinal margin sloping and nearly straight; anterior end deeply excavated under the beaks, rounded below, and merging by a regular curve into the base, which is broadly convex, and most prominent in the middle; posterior end obliquely truncated; a strongly marked subangular ridge runs from the beaks to the posterior basal angle, behind which the surface is slightly concave. Surface marked by very minute lines of growth.

Length, .52 ineh; width, .44 ineh; diameter through both valves, .38 ineh.

Not rare at a single loeality in the vieinity of Cottonwood Creek, from either the Chieo or Shasta Group. Mr. Mathewson colleeted a eonsiderable number of speeimens in 1865, from a gray roek, different from any bed with which $I$ am aequainted, and which seems to have yielded no other speeies. The shell is of extraordinary thinness and delieaey, and is so transparent that, in some cases, its presenee ean only be deteeted by the opaeity of the thickened eardinal margin. On aecount of its extreme delieacy, I have not even attempted the futile task of exposing the hinge, and have referred it doubtfully to the above genus, from its general appearanee, rather than from any charaeter that it possesses. 


\title{
CARDIUM, Linn.
}

\section{C. (Lavicardium) annulatum, Gabb.}

\author{
Pl. 30, Fig. 81.
}

(C. (Lavicardium) annulatum, Gabb ; Pal. Cal., Vol. 1, p. 171, pl. 23, fig. 152.)

This beautiful and delicate shell was described and figured from a distorted specimen. Since the publication of Volume 1, numcrous specimens have been obtained by Mr. Mathewson at Martinez, and I am cnabled to correct a point in the description, and to give a more accurate figure of its form. The posterior side, instead of being regularly convex, is slightly truncated obliquely.

It is characteristic of the Chico Group, and seems peculiar to a series of bard beds of this group found at Martinez, south of Mount Diablo, and in Orestimba Cañon, in the same range, further south.

\section{C. (Protocardium) translucidum, n. s.}

Pl. 30, Fig. 82, $82 a$.

SheLL small, thin, gibbous, rounded subquadrate; beaks prominent, incurved; ends nearly equal, the anterior rounded, posterior rounded subtruncate; base broadly rounded, a little more prominent in advance than behind. Surface marked by a few small, regular, rounded, radiating ribs on the posterior side, and by concentric lines of growth.

Length, . 3 inch; width, .3 inch; diameter, .23 inch.

From the same bed as the preceding, Martinez.

Resembling C. (P.) Placerensis, this shell differs in its outline, being more quadrate, in the posterior radiating ribs being more regular and numerous, and in the entire absence of the large rib on the margin of the costate area, the marginal rib in this species differing in no respect from the others.

\section{CARDITA, Brug.}

\section{Hornit, Gabb.}

Pl. 30, Fig. 83, $a$.

(Cardita Hornii, Gabb; Pal. Cal., Vol. 1, p. 174, pl. 24, fig. 157.)

(Cardita planicosta, Con. (not Lam.); P. R. R. Rep., Vol. 5, p. 321, pl. 2, fig. 6.) 
This shell, called by Mr. Conrad "the finger-post of the Eocene," proves to be the "finger-post" of the Tejon Group. Like its ally of the Eocene, it is peculiar to the formation, and its geographical limits are coextensive with the group.

At the time that I described it I had a pretty large series of specimens from near Fort Tejon, Martinez, and Clayton. Since then it has been found in several other localities, and fine specimens have been obtained near New Idria, and west of Griswold's, on the road from the latter place to San Juan. I had, however, no specimens of the true planicosta for comparison, and was obliged to depend partly on figures, partly on my memory, as I mentioned in the note to the description. That description. should be slightly modified, so as to read-"Shell variable in shape, subtriangular to subquadrate," the subquadrate forms usually being the more adult, though some specimens, four and four and a half inches across, are as distinctly triangular as the typical planicosta. The number of the ribs is as constant as is usually the case in costate shells. I have before me one specimen with twenty-one, another with twenty-three ribs. I have compared my specimens with shells from the London Clay, and from the Alabama Eocene, and find that, except in the extrenie quadrate forms, they are absolutely identical in all characters save one. The hinges are so similar that $I$ despair of making an intelligible written description of their minute differenees, and should hardly feel willing to trust an artist with their delineation. But a character exists by which the merest tyro could separate the two species. It is in the shape of the cross-section of the ribs. The surface is well represented by the figure in the first volume, and I have only to append a series of outline cross-sections to illustrate this difference. Fig. 83 is a magnified section of the ribs in their normal state; $83 a$, is a section of these ribs after laving been corroded by atmospheric or other causes; while $83 b$ is a section of the ribs of $C$. planicosta. It will be observed that even when the ribs of $C$. IIornii liave been flattened by weathering, they are still different from the Eocene shell, in their slight elevation, and in the wide interspaces. Some old specimens of $C$. Hornii have the same peculiarity as some old specimens of $C$. planicosta, in the ribs becoming more flat, and even nearly obsolete towards the base. This is not a constant character in either species. Onc fine large shell of the latter form, from Claiborne, Ala., in the museum of the Philadelphia Academy, has the ribs as sharply defined at the base as towards the beaks.

\section{CLISOCOLUS, $N$. Gen.}

Pl. 30, Fig. 84.

SHeLl thin, Lucinæform, without a lunule; ligament external, lodged in a deep groove; hinge edentate, having in the right 
valve a slight thickening under the beak, and in the left valve an obsolete tubercle; pallial line unknown; internal margin entire.

I propose this genus on the shell described in the first volume of Pal. Cal., as Loripes dubia. The figures there given of the hinge are not good, the engraver not having faithfully followed my drawings. The tubercle in the left valve especially, is represented as too well-defined. The genus secms to be a member of the Lucinida, though from our ignorance of the shape of the mantle margin, the ultimate reference must yet remain an open question.

C. Dubius, Gabb.

(2. Loripes dubia, Gabb ; Pal. Cal., Vol. 1, p. 127, pl. 74, fig. 170, 171.)

Peculiar to the Chico Group.

\title{
LUCINA, Brug.
}

The references to the plates of $L$. nasuta and $L$. postice-radiata, in Vol. 1 , were transposed, by mistake, in the text. They should rcad, L. nasuta, Pl. 24, Fig, 159, and $L$. postice-radiata, Pl. 24, Fig. 158. By reading the descriptions, the error becomes at once obvious.

\section{CRASSATELLA, Lam.}

\author{
C. Grandis, Gabb.
}

(C. grandis, Gabb ; Pal. Cal., Vol. 1, p. 181, pl. 24, fig. 163.)

(C. alta, Con.; Pacific R. R. Report, Vol. 5, p. 321.)

(C. alta, Con.; Smithsonian Check List, Eocene, No. 104.)

(Not C. alta, Con.; Tert. Foss., p. 21, pl. 7.)

In my description of this species, I pointed out sufficiently the specifie differences between the two forms. Another character, however, has since becn discovered in the lunule, with which $X$ was then unacquainted. In $C$. alta it is deeply impressed, the sides being vertical, the base flat, and the sides forming right angles both with the adjoining portion of thesurface and with the floor of the depression. In $C$. grandis the lunule is equally deep and longer, but its margin is rounded, and the sides convergent, there being no flat space at the base. 


\section{COMPacta, n. s.}

Pl. 30, Fig. 85.

SHELL small, elongate subtrigonal, thiek; beaks about a third of the length from the anterior end; eardinal margin sloping with a slight eonvexity to the posterior end, whieh is narrowly truneated; anterior end sloping, nearly straight above, broadly eonvex and prominent below; base nearly straight posteriorly, rounding upwards regularly in advanee, and most prominent under the beaks; the lunule is almost entirely hidden in the matrix, but appears to be large and deeply impressed. Surfaee polished and marked by lines of growth in the middle, whieh develop into small ribs anteriorly; an angular ridge runs from the beaks to the posterior basal angle.

Length, .95 inch; width, .68 inch; height of single valve, .2 inch.

A single valve, from the Martinez Group, Martinez, given me by Mr. Mathewson.

This shell might be mistaken for the young of $C$. grandis, but it differs in being more elongate posteriorly, in the possession of the marked angular ridge, in the square posterior truncation, and in its greater convexity.

UNIO, Retz., Auct.

\section{U. Ifubbardir, n. s.}

Pl. 30, Fig. 86.

Sheld moderately large, thiek, obliquely subquadrate; beaks anterior, about a fourth of the length from the anterior end, somewhat prominent; posterior cardinal border straight (?) and slightly sloping (from lines of growth on the younger portions of the shell); anterior end regularly rounded and somewhat prominent; posterior end obliquely truneated; base nearly straight and sloping behind, most prominent under the beaks, and curving upwards regularly in advanee; a strong angular ridge runs obliquely downwards and backwards from the beaks to the posterior basal angle. Surface marked by eoarse, irregular lines of growth. 
Length, 3. inches; width, 1.8 inch; thickness through both valves, 1 inch.

A single specimen, from the Nanaimo Coal Mine, Vancouver Island, Chico Group; kindly loaned me by Mr. Samuel Hubbard, of the Pacific Mail Steamship Company of San Francisco, and to whom I dedicate the specics, in recognition of the unostentatious but valuable scrvices he has been rendering to science for a series of ycars past.

\title{
MYTILUS, Linn.
}

M. QUADRATUS, n. s.

Pl. 31, Fig. 87.

(Inoceramus Piochii (pars), Gabb; Pal. Cal., Vol. 1, pl. 25, fig. 174.)

SHELL moderate in size, thin, flattened, elongaté, rounded quadrate; beaks small, acute, terminal; anterior margin very slightly sinuous above, and broadly convex, nearly straight below; posterior side slightly convex and nearly parallel with the anterior; cardinal margin slightly sloping, and uniting by a broad curve with the posterior; base broadly convex, most prominent anteriorly. Surface slightly but regularly convex, except close to the anterior margin, where it falls more rapidly; ornamented by a few irregular lines of growth.

Figures, natural size.

From the Chico Group, at Martinez and Tuscan Springs.

In my original description of "Inoceramus" Piochii, I supposed this to be the smaller valve of that species, and accordingly included it. As will be seen further on, however, both valves of that shell are very different from the present one.

The present species can be at once distinguished from all other Mytili by its unusually square form. Its nearest ally is $M$. ascia, but that shell is arched, and the more slender form and acute anterior end will sufficiently separate them.

\section{MODIOLA, Lam.}

\author{
M. MAJOR, n. s.
}

Pl. 31, Fig. 88.

SHell very large, thick, slightly curved; beaks small, about an eighth of the length from the anterior end, which is narrowly 
rounded and produced; cardinal margin slightly arched; base concave in the middle, convex posteriorly; posterior end irregularly convex, most prominent below. .Surface marked by coarse, irregular lines of growth.

Length, 4.8 inches; width, 2.4 inches; diameter about 1.5 inch.

Common in the white limestone of the Shasta Group, east of Knoxville, Lake County; also found smaller in the same beds in Morgan Valley, south of Clear Lake, and at the Hot Sulphur Springs, east of Clear Lake, Colusa County.

A fine species, well characterized by its size, being the largest species of the genus, with which I am acquainted. Some specimens are over six inches in length.

\section{MELEAGRINA, Lam.}

M. Antiqua, 11. s.

Pl. 31, Fig. 89.

Shell small, flattened, rounded quadrate; beaks acute, produced, and terminal; cardinal margin straight, very slightly sloping; anterior margin strongly sinuous for a short distance under the beaks, continued in a regular curve, running into the base, which is a little the most prominent posteriorly; posterior margin nearly straight, uniting with the base by a more abrupt curve than that on the anterior side. Surface plain, or marked only by lines of growth. Hinge slender; muscular sear large, broadly reniform, placed a vcry little posterior to the middle.

Length, 1.3 inch ; width, 1.5 inch; thickness of two valves, .32 inch.

From Departure Bay, Nanaimo, Vancouver Island, associated with Trigonia Evansana, Pecten Traskii, and other fossils equally characteristic of the Chico Group.

The oldest known species of the genus, being from a deposit certainly older than the white chalk. It possesses all of the generic characters in a much more marked manner than we might fcel warranted in expecting, when we consider how far rcmoved the species is from the geological age in which the genus has obtained its greatest specific development. It is usually the case that, where a genus attains its greatest numerical specific development in any one geological age, those species which first appear, especially if in a remote formation, as well as those that mark 
its gradual decline before its final disappearance, are the most apt to present variations from the normal type of the genus.

\section{INOCERAMUS, Sby.}

\section{Elliotit, n. s.}

Pl. 31, Fig. $90 a$.

SheLl large, equivalve, subquadrate; beaks terminal; cardinal margin straight,(?) nearly as long as the shell; anterior cnd truncated, nearly straight; posterior end straight and sloping outwards above, rounded below, and, with the base, forming a nearly perfect semicircle. Surface marked by about a dozen large, sharp, concentric, imbricating ribs or lamellæ, with broad concave interspaces.

Figure, natural size; from a cast in sandstone: the accompanying outline section of the surface is restored from impressions in the matrix.

From the semi-metamorphosed Cretaceous sandstone on Alcatraz Island, San Francisco harbor. Presented to the survey cabinct by Major Gcorge H. Elliot, U. S. Engineers, by whom the first specimens were discovered. On subsequently visiting the locality, I found numerous casts of this and of several other bivalves, the latter in too inperfect a condition to be recognized. The species is of unusual interest, being the first incontestable proof discovered, of the Cretaceous age of the long-disputed "San Francisco Sandstone."

\section{WhitNeyi, n. s.}

Pl. 32, Fig. 91.

Shell large, elongate, subelliptical; beaks large, prominent, anterior, subterminal; cardinal margin straight; anterior end and base broadly, regularly, and continuously rounded; postcrior end forming a curve of longer radius, but also continuous with the base. Surface marked by about sixteen large concentric ribs, the basal margin being abruptly truncated at a nearly right angle with the remainder of the surface; details of surface ornament unknown.

PAL. VOL. II. -26 
Length, 3.3 inches; width, 2.8 inches; height of single valve, .8 inch

A single cast in a soft, gray, finc-grained sandstone, from near Folsom, and probably of the age of the Chico Group. Found by Professor Whitney. I have seen in this same matrix, Ammonites Chicoensis, A. Suciaensis, Baculites Chicoensis, and casts of some undetermined Gasteropods.

\section{AUCELLA, Keyserling.}

\section{A. Prochir, Gabb.}

Pl. 32, Fig. 92, $a, b, c$.

(Inoceramus Piochii, Gabb ; Pal. Cal., Vol. 1, p. 187, pl. 25, fig. 173 (exclus. fig. 174).)

This shell was originally described from two or three small specimens from near Mount Diablo; the Tuscan Springs specimens being the Mytilus, figured at 174, and which $I$ have separated in the present paper. During the last three or four years, the species has becn discovered in profusion in a large number of localitics, and proves to be of grcat valuc in the idcutification of one of the members of the Shasta Group. Its specific characters may be defined as follows:

Shell variable in outline, couvex, variably inequivalve ; symmetrical and obliquely ovate in the young state, when both valves have a small, narrow ear on the posterior side, which becomes obliterated as the shell grows larger. In the adult, large valve long, narrow, tapering, irregular in form; beak large, and strongly incurved, often overhanging the opposite valve. Smaller valve more or less triangular, anterior side usually somewhat truncated; beak small, acuminate and pointed in advance; posterior cardinal margin pretty regularly arched and sloping downwards rapidly; under the beak, on the anterior face, is a deep indented fold of the shell, forming an internal sinus (seen in figs. 92 , and $92 \mathrm{c}$ ). Surface of both valves very variable in convexity, and in the adult, covered by irregular wrinkles, besides fine, and in some cases pretty regular, concentric ribs.

Figures, natural size, except $92 c$, which is twice the size of the original.

Very characteristic of a series of shalcs of the Shasta Group, found from Mount Diablo, at various points along the cast face of the Coast Range to the north end of the Sacramento Valley. Two or three good spccimens from Washington Ter- 
ritory, east of Puget Sound, were presented by Mr. Samuel Hubbard to the California Academy of Natural Sciences. In Colusa County, east of Clcar Lakc, I found this shell forming almost the entire bulk of some bers, interstratified with the white limestones. The Mount Diablo specimens are rare, and always small, while some from Colusa and Lake Counties are nearly three inches in grcatest length. The only species that I have cver found associated in the samc strata with the present one, is Belemnites impressus, which I liave found at almost every locality of the Aucella, and which is also very characteristic of the most typical beds of the Shasta Group, in the vicinity of Cottonwood Creek.

\section{PINNA, Linn.}

\section{P. BreweriI, Gabb.}

P1. 32, Fig. 93.

(P. Brewerii, Gabb ; Pal. Cal., Vol. 1, p. 188, pl. 25, fig. 175.)

This is a rare species, and I have had the opportunity of examining but few specimens. A very fine one now before me, and for the loan of which I am indebted to the kindness of $\mathrm{Mr}$. Voy, of Oakland, exhibits the specific characters better than any other yet discovered. The angle of divergence of the sides in this case, is nearer $40^{\circ}$ than $20^{\circ}$. Near the middle of each valve is a strongly marked rib, on one side of which are six or seven fine, slightly elevated, radiating ribs; on the other sidc are two or three similar, obsolete radiations, and starting from the margin are some stronger, but irregular undulations, parallel with the midrib, which run downwards and enrve inwards towards the middle; from the lines of growth, the base of the shell seems to have been notched in the middle.

The figure is natural size. Found only in the Chico Group; the present specimen being from Shasta County. 


\section{TRIGONIA, Brug.}

T. IQUUICOSTATA, n. s.

(Indet.; Pal. Cal., Vol. 1, pl. 26, fig. 198.)

SHell small, trigonal, elongate; beaks prominent, anterior; cardinal margin sloping posteriorly, straight to slightly convex or concave; anterior end regularly rounded; base broadly rounded, most prominent in the middle; posterior end narrowly truncated. Surface marked by regular, square, concentric ribs, with equal, flat, interspaces; these ribs end abruptly in advance, and are continued by fine lines starting with an angle somewhat less than a right angle; posteriorly there is an angular ridge running from the beak to the posterior basal angle, and on crossing this the ribs curve upwards towards the margin. Internally, the surface is marked by four or five radiating ribs under the beak; this character is not always present; the hingc teeth are unusually large, arc coarsely ribbed, the anterior tooth being supported longitudinally by a vertical plate, starting from the upper edge of the muscular scar.

Figure in Vol. 1, natural size.

Common in the Chico Group at Orcstimba Cañon, Curry's, south of Mount Diablo, Jacksonville, Oregon, and found occasionally at Martinez, and Cottonwood Creck.

\section{AXIN $\mathbb{E A}$, Poli.}

A. sagittata, Gabb.

(A (? Limopsis) sagittata, Gabb ; Pal. Cal., Vol. 1, p. 197, pl. 31, fig. 267.)

At the time that this species was described, I was unable to decide finally as to its generic relations. I have since been enabled to expose the area, which is very narrow but characteristic, and which places it in the above genus. 


\title{
NUCULA, Lam.
}

\author{
N. (Acila) truncata, Gabb. \\ (Nucula truncata, Gabb; Pal. Cal., Vol. 1, p. 198, pl. 26, fig. 184.)
}

A very common species, found at almost every locality of the Chico, Martinez, and Tejon Groups. The specific identity of these fossils having been denied, I have re-investigated the subject with the utmost carc, and have failed to find even a varietal difference between specimens from the threc deposits. At the same time, in the Martinez Group, at Martincz, in addition to the normal form, and associated with it, is a larger variety, of which $I$ have three specimens. These 1 have compared, with the assistance of Mr. Conrad, both with the smaller varicty of the Martinez Group, and with others from the adjoining deposits, and we can find no difference except the size.

\section{N. SOLITARIA, n. s.}

Pl. 32, Fig. 94.

SHeLl small, rounded triangular; beaks small, rather prominent, subterminal, and inclined forwards; cardinal margin nearly straight, rapidly sloping posteriorly; anterior end subtruncated, a little excavated under the beaks, and very slightly convex below, united with the base by a pretty well-marked angle; base rounded, most prominent a little behind the middle; posterior end narrowly rounded. Surface marked by a few large and many minute lines of growth; hinge robust, pit small.

Length, .26 inch; width, .22 inch; height of single valve, $.04 \mathrm{inch}$.

Very rare in the Chico Group, Texas Flat, Placer County.

With the general form of $N$. (Acila) truncata, this shell is very much smaller, and the entirc absence of the radiating ornament will at once distinguish it. It is wider from beak to base in proportion to its length, the anterior end is much more convex, and the posterior is somewhat narrower.

\section{LEDA, Schum.}

\section{Gabbir, Coll. sp.}

(L. protexta? Gabb ; Pal. Cal., Vol. 1, p. 199, pl. 26, fig. 185.)

(Not L. protexta, Gabb; Jour. Phil. Acad., 2 Ser., Vol. 4, p. 303, pl. 48, fig. 23.)

(Nuculana Gabbii, Con.; Smithsonian Check List, No. 44.) 
On eomparing the Californian with the New Jersey speeies, I find that there are differenees in form suffeient to warrant their separation. Mr. Conrad having proposed a new name for the Western shell, I am obliged to adopt it, though I should have preferred his ehoosing some other appellation. The present species is broader in proportion to its length, than the casts in the New Jersey marl, whieh, though all more or less imperfeet, seem to have been remarkably long and straight.

\section{PECTEN, Brug.}

\section{P. Traskir, Gabb.}

Pl. 32, Fig. 95.

(Pecten Traskii, Gahb; Pal. Cal., Vol. 1, p. 200, pl. 26, fig. 187.)

This rare shell was originally known by but two spccimens from the loosegrained sandstones of Texas Flat, Plaeer County. A third has sinee been found by myself in the greenish roek on the north shore of Departure Bay, near Nanaimo, Vancouver Island, and which throws some additional light on the specifie eharaeters. The shell is very thin, and the surface, of which a fine fragment is preserved, has a slightly different ornament from the figure in the first voiume. The lines in the interspaces between the ribs, whieh I mentioned at the time as being represented too numerous in the tigure, are elevated threads, slightly irregular in their direction, and radiate from the middle. The figure above quoted represents their character near the side of the shell. The present figure represents the middle of the surfaee. The imbrieation of the ribs is produeed by these fine lines erossing them. So far as we know at present, the speeies is confined to the Chieo Group.

\section{P. Martinezensis, n. $\mathrm{s}$.}

Pl. 33, Fig. 96.

Sheld minute, thin, elongate, equivalve, equilateral; sides straight above, below forming with the base a regular eurve. Lower valve, right auricle deeply emarginate, narrow; left smaller. Upper valve, right auriele small; left large, margin slightly sinuous. Surface ornamented by numerous fine radiating ribs.

Length from beak to base, .34 inch.

Not common in the Martinez Group, Martinez.

From the other Pectens of the California Cretaeeous, this species can be distinguished by its small size and radiating ribs. I was formerly inclined to believe 
it to be the young of $P$. Traskii, but have now three specimens varying only in size one or two-hundredths of an inch, and consequently cannot resist the belief that it is a distinct species.

\section{P. COMPLEXICOSTA, n. s.}

Pl. 33, Fig. 97, 97 a.

Shell moderate in size, thin, equivalve, nearly equilateral; sides and base forming a regular curve, slightly elongated; the right side of the upper valve, and corresponding side of the lower, a little the most convex above; upper valve with the left ear a little concave on its lateral margin; shape of the right ear unknown; right ear of lower valve narrow, produced, and deeply emarginate. Surface marked by about twelve or fourteen radiating ribs, with sometimes an equal number of smaller ones intercalated; these are more strongly marked on the cast than externally; besides the ribs, the entire surface is closely sculptured by minute radiating lines, very variable in size.

Figure, natural size, and a magnified view of the surface.

Common in the white limestone of the Shasta Group in Morgan Valley, south of Clear Lake, collected by Professor Whitney. Although the species is abundantly represented at this locality, no entirc specimens have ever becn obtained, owing to the character of the matrix. By the study of a large number of fragments, I have been enabled to arrive at all the important specific characters, except the shape of the ear, which is represented broken in the figure, as it occurs in the best specimen. Two or three casts before me give the outline, and the details of the surfaee are drawn from impressions in the matrix.

\section{P. interradiatus, n. $\mathrm{s}$.}

Pl. 33, Fig. 98, $98 a$.

Shell small, subcircular, equivalve, equilateral, compressed, thin; upper valve, ears equal, moderately large; lower valve, right ear long, deeply and narrowly emarginate. Surface marked by very numerous fine radiating lines, and obscure lines of growth. Internal surface of both valves bearing eight straight, equidistant, large ribs, of variable length among themselves, extending 
from the beaks to near the middle of the shell, and ending abruptly.

Figures, natural size.

From a buff-colored shale east of New Idria, at or very near the summit of the Tejon Group, where $\mathbf{I}$ found it very abundant.

$P$. opereuliformis and $P$. Californicus of the California Cretaceous are marked by concentric lines only; the former is much larger than the present species, and the ears are of a very different shape; neither of those species has the internal ribs of this shell. The other species are longer, with smaller ears, and are markedly costate.

\section{NEITHEA, Drouet.}

N. grandicosta, n. s. Pl. 33, Fig. $99 a$.

Shell minute, very inequivalve, equilateral, elongated; lower valve, sides tapering a little concavely; general contour of the base semicircular, with six prominent angles and concave interspaces. Surface marked by six very large, round, equidistant ribs, the interspaces carrying a small rib, flanked on each side by one still smaller; in some cases the sides of the large ribs are faintly striate; crossing these ribs are minute, very regular lines of growth; ears very unequal, the right ear long and deeply emarginate.

Length from beak to base, .5 inch.

Rare in the Shasta Group, Cottonwood Creek, Shasta County.

This is one of the smallest, if not absolutely the smallest species yet described in the genus. It has some resemblance to $N$. duplicicosta, Roem. sp., of Texas, which has three intermediate ribs; but in that shell the large rib is very compound. It is most nearly allied to the French Neocomien species, N. atava, d'Orb. sp., but the ears are of a different shape; in that shell the right ear of the lower valve is much smaller, the interspaces are more concave, and the large ribs, instead of being simple, are made up of three smaller ribs. 


\section{LIMA, Brug.}

\section{Shastaensis, 11. s.}

Pl. 33, Fig. 100.

SHell small, conpressed, irregularly subelliptical, equivalve; anterior side and base forming a regular curve, the posterior margin of the base rounding up rather abruptly to the posterior side, which is straight; ears very small, the anterior triangular, the posterior narrow, almost linear; posterior umbonal slope very narrow. Surface ornamented by twenty or twenty-one prominent, straight, radiating ribs, with the interspaces of equal size.

Greatest length, from beak to base, .6 inch; antero-posterior diameter, $.45 \mathrm{inch}$; depth of single valve, .09 inch.

Rare in the Shasta Group, Cotton wood 'Creek, Shasta County.

I have two specimens of this shell, one of which is somewhat mutilated, but they fortunately show both valves. The species is less oblique than $L$. microtis, and the ornament of the surface is entirely different, as it is also from $L$. appressa. The latter species is narrower above than the present one, it is a straighter shell, and the anterior ear is larger.

\section{MULTiradiata, n. s.}

Pl. 33, Fig. 101.

There is but a single mutilated specimen of this fine shell in the collection, with its corresponding impression in the matrix. The lines of growth on the latter, together with some, less distinct, on the shell itself, supply the outline, exccpt of a small portion of the posterior margin, and of the ear on that side. The specific characters, so far as shown, are as follows:

Shell (right valve) large, oblique, compressed subelliptical; anterior side forming a regular curve to the middle of the base, very slightly sinuous above, under the ear; posterior portion of the basal margin much more narrowly rounded than in advance, uniting by a broad curve above, with the posterior side, the upper half of which seems to have been nearly straight; the anterior ear seems to have been long and narrow. Surface convex a little PAL. VOL. II. -27 
behind the middle, the anterior slope being broad and nearly flat; the entire surface covered by very numerous, slightly wavy, somewhat irregular, radiating ribs of small size, and with narrow interspaces; on the younger portion of the shell, the spaees between the ribs are mere impressed lines, the ribs being eonvex; near the base, however, the ribs become proportionally smaller, the spaces are wider than the ribs, and are eoneave, and the whole are crossed by minute subsquamose lines of growth.

Length from beak to base, of a broken speeimen, 3 inehes.

From the vicinity of Lower Lake Village, Lake County ; from a deposit intermediate between the Martinez and Tejon Groups.

This slell belongs to the type of Sowerby's Plagiostoma, a group of extinet species, well characterized by their peeuliar surface ornament. I am familiar with the external characters of a number of the speeies, but have never had the good fortune of being able to examine the hinge.

The present species is closely allied to $L$. microtis, nobis, but is proportionally mueh broader, the posterior side is straighter, the anterior side is mueh more regularly eurved, being most prominent in the middle or below, while in microtis the greatest prominence is just below the ear; the most marked differenee seems to be in the relative degree of obliquity of the two spceies. Another differenee is in the surfacc ornament; both have numerous, small ribs, but microtis has the interspaees punetate and the ribs flat, while the present shell has round ribs, and the grooves are perfeetly simple, except for the lines of growth.

\section{ANOMIA, Linn.}

\section{A. Vancouverensis, n. s.}

Pl. 33, Fig. 102.

ShelL eireular, thin; upper valve unknown; lower valve flat; marked by strong lines of growth and by very faint radiating lines; aperture elongated, oblique, oeeupying nearly a third of the diameter of the shell.

Diameter, 1. inch.

From the Chieo Group, Departure Bay, near Nanaimo, Vaneouver Island.

It is not impossible that this may prove to be the under valve of $A$. lineata, nob. (Vol. 1, p. 203, pl. 26, fig. 193), which belongs to the same group in the formation, and of whieh the lower valve was unknown. 


\title{
OSTREA, Linn.
}

\author{
O. Idriaensis, n. s. \\ Pl. 33, Fig. 103, $b, c, d$. \\ Pl. 34, Fig. 103, $103 a$.
}

Shell moderately large, oblique, often curved, heavy; lower valve usually deep, more oblique than the upper; hinge straight or deflected to the left, median groove pretty strongly marked; internal margins in the young shell, finely crenulated or pitted towards the beak, this character disappearing to a grcat extent in the adult. Surface of both valves roughly and very irregularly squamose. Muscular scar reniform to semicircular, and placed distinctly to one side of the middle.

Length, from three to six inches. The figures are all natural size, the greatest thickness of the specimen, fig. 103, is 1.25 ineh, the fragment, $103 a$, has about an equal thickness, indicating a shell when entire, perhaps three inches through both valves.

From the Tejon Group, on the same horizon as the beds at Marsh's, near Mount Diablo. Locality, about two miles east of the Hacienda at New Idria, where it is very abundant.

The obliquity of the hinge in this shell is quite a variable character. It is well represented by the figures 103 and $103 d$, in its greatest extent; figure $103 a$ is a transition form, while a specimen of an upper valve before me, has the hinge nearly straight.

\section{O. APPRESSA, n. . \\ Pl. 34, Fig. 104, $104 a$.}

SHeLc thin, flat, more or less equilateral, valves nearly equal, usually about two-fifths longer than wide. Surface covered by numerous, thin, squamose plates. Hinge flat, large; margins simple, sometimes subsquamose. Muscular sear small, oblique.

Figures, natural size. Some specimens were found seven inches long.

$I$ found this shcll forming a stratum several feet thick, adjoining a bed of coal, and in associated strata were familiar speeies eharacteristic of the Tejon Group. 
The locality is on one of the branches of Eel River, at the mouth of Salt Creek, soutlrwest of Round Valley, Mendocino County

The species is remarkablc for its thin, flat shell, often distorted, and for its broad, flat hinge.

\title{
B RACHIOPODA.
}

\section{RHYNCHONELLA, Fisch.}

\section{R. Whitneyi, Gabb.}

\author{
Pl. 34, Fig. 105, $a, b$.
}

(Terebratella Whitneyi, Gabb ; Pal. Cal., Vol. 2, p. 35, pl. 12, fig. 62, 62 a.)

(Compare R. peregrina, Von Buch sp., d'Orb.; Pal., Fr. Terr. Cret., Vol. 4, p. 16, pl. 193.)

When this species was described, it was supposed to be Tertiary, and the description and figures werc taken from immature specimens. In the latter part of 1866 I had an opportunity of studying the rocks from which it came, and found then intcrstratificd with shales containing Aucella Piochii and Belemnites impressus, thereby proving them to belong to the Shasta Group. The species is extremcly abundant in the white limestones of Lake and Colusa Counties, and I was fortunate in procuring the large specimen now figured. The specics is very closely allied to $R$. peregrina of the French Neocomien, and it is not impossible that they may yet prove identical. I shall, however, retain the specific name for the present, until the question of their identity can be definitely settled pro or con. The following points of difference exist between the specimen before me, and a fine example of the French shell in the Museum of the Philadelphia Academy. While the number and shapc of the ribs are the same in both specinens, the Californian shell is very much more convex, the beak is higher, and the deltidium is markedly narrower; in $R$. peregrina, all of the ribs which reach the lateral margins cause a strong zigzag serration of the margin when viewed directly; in the present shell, this effect is produced only below the middle, on account of the upper ribs becoming less distinct as they approach the edge.

Very numerous specimens of another Brachiopod have been found by Mr. Mathewson at Martinez, in a sandstone, probably of the Chico Group The valves are always separated, and $I$ have never succeeded in discovering a single generic character. The shell is very transverse, a third wider than long, and is closely covered by minute radiating ribs to beyond the middle, where, reversing the usual order, instead of branching they unite by twos or threes, forming a few largc angular folds on the lateral and basal margins. One valve appears to havie a broad 
shallow sinus at the middle of its base, bcaring thrce or four of these folds; a corresponding tongue or prolongution of the other valve meeting it, but without the usual median depression on the surface. Among between twenty-five and thirty specimens, not one shows all the surface of a valve, though by counting several, it seems that there are nearly sixty of the small ribs and about fifteen of the large marginal folds.

\section{R A D I A T A. \\ SMILOTROCHUS, E. \& H.}

? S. CURTUS, n. s.

Pl. 34, Fig. 106, 106 a.

Polypidom circular, low, base apparently rounded or very blunt, sides but slightly diverging. Internal septa short, numerous, leaving a well-marked cup-like cavity in the middle; their sides marked by numerous small, curved, raised lines, radiating from below upwards and inwards. External surface unknown.

Figures, natural size.

Rare near Martinez. Two specimens in my collection from either the Martinez or Chico Groups; found by Mr. Mathewson. This coral is easily distinguished by its short, robust, cup-like form. The surface sculpture is lost on both the specimens, being torn away by the hard matrix in which they were embedded. 



\title{
PALEONTOLOGY OF CALIFORNIA.
}

\author{
V 0 L. I I.
}

SECTION II.

C R E T A C E O US FO S S I S.

P A R T I I.

SYNOPSIS OF THE CRETACEOUS INVERTEBRATE FOSSILS OF CALIFORNIA. 



\section{CRUSTACEA.}

Callianassa, Leach. C. Stimpsonit, Gabb.

C. Stimpsonii, Gabb ; Pal. Cal., Vol. 1, p. 57, pl. 9, fig. 1, $a, b$, c.

Id., Gabb; Vol. 2, p. 127, pl. 19, fig. 3.

Chico Group, Chico Creek; Tejon Group, Clayton and Tejon.

GEN? SP. INDET.

A carapace from the Martinez (.?) Group, Martinez.

GEN? SP. INDET.

Hands of a Macrouran, from the Shasta Group, Cottonwood Creek, Shasta County.

\section{OLLUSCA.}

CEPHALOPODA.

PtIloteUthis, Gabb. P. Foliatus, Gabb.

Ptiloteuthis foliatus, Gabb ; Pal. Cal., Vol. 2, p. 128, pl. 19, fig. 4.

Shasta Group, Cottonwood Creek.

BELEMNITES, Cuv. B. IMPRESSUs, Gabb.

B. impressus, Gabb; Vol. 1, p. 58, pl. 9, fig. 2.

Shasta Group, Cottonwood Creek, Horsetown, Mount Diablo, Colusa County.

NAUTilus, Brug. N. Texanus,? Shum.

N. Texanus, Shum.; Trans. St. Louis Acad., 1860, p. 590.

Id., Gabb; Pal. Cal., Vol. 1, p. 59, pl. 9, fig. 3.

? Shasta Group, Cottonwood Creek, Alderson's Gulch, Mount Diablo.

PAL. VOL. II. -28 . 


\section{N. Campbelli, Meek.}

N. Campbelli, Meek; Proe. Phil. Aead., 1861, p. 318.

Comax, Vaneouver Island.

atdRIA, Bronn. A. Mathewsonit, Gabb.

A. Mathewsonii, Gabb; Vol. 1, p. 59, pl. 17, fig. 31.

Martinez Group, Martinez; Tejon Group, Clayton and Tejon.

\section{AMrimonites, Brug. A. BreweriI, Gabb.}

A. Brewerii, Gabb; Vol. 1, p. 62, pl. 10, fig. 7 .

Id., Gabb; Vol. 2, p. 130, pl. 20, fig. 5 ; pl. 19, fig. $5 a, b, 6 a$.

A. Newberryanus, Gabb, part. (not Meek); Vol. 1, pl. 10, fig. 6.

Shasta Group, Cottonwood Creek.

\section{A. Haydenit, Gabb.}

A. Haydenii, Gabb; Vol. 1, p. 62, pl. 10, fig. 8 .

With the preeding.

\section{A. Peruvianus,? Von Buch.}

A. Peruvianus, Von Bueh; Petrif. Ree. par Humboldt en Amer., p. 5, pl. 1, fig. 5-7.

A. Peruvianus? Gabb; Vol. 1, p. 63, pl. 10, fig. 9.

Id., Nareou; Geol. North Amer., pl. 5, fig. 1.

From Von Buch's original figure and deseription, it seems doubtful if his shell is really an Ammonite, but my single fragment is too imperfeet to permit more than a very doubtful determination.

Chico Group, Tusean Springs.

\section{A. Traskit, Gabb.}

A. Traskii, Gabb; Vol. 1, p. 63, pl. 11, fig. 10; pl. 12, fig. 11.

Id., Gabb ; Vol. 2, p. 131, pl. 19, fig. 7, $7 a, 7$ b.

Shasta Group, Cottonwood Creek, and Arbuekle's Diggings, Shasta County.

\section{A. Ramosus, Meck.}

A. (Scaphites) ramosus, Meek; Trans. Albany Inst., Vol. 4, p. 45.

A. ramosus, Gabb; Pal. Cal., Vol. 1, p. 65, pl. 11, fig. 12, $12 a$; pl. 12, fig. $12 b$. Shasta Group, Cottonwood Creek. 


\section{A. Hoffmannit, Gabb.}

A. Hoffmannii, Gabb; Vol. 1, p. 65, pl. 11, fig. 13, $13 a$; pl. 12, fig. $13 b$. $I d$., Vol. 2, p. 131, pl. 20, fig. 8, 8 a.

Shasta Group, Horsetown, and Cottonwood Creek.

\section{A. Rémondi, Gabb.}

A. Rémondii, Gabb; Vol. 1, p. 66, pl. 12, fig. 14, 14 a, 15. Shasta Group, Cottonwood Creek; Chico Group, Pence's.

\section{A. Batesir, Trask.}

A. Batesii, Trask ; Proc. Cal. Acad., 1855, Vol. 1, p. 40.

A. Batesii, Gabb; Vol. 1, p. 67, pl. 13, fig. 16, 16 a.

A. Batesii, Gabb; Vol. 2, p. 132, pl. 20, fig. 9, $9 a$; pl. 21, fig. 10, $a, b$.

Shasta Group, Cottonwood Creek, Chico Group, Benicia, and south of Mount

Diablo.

\section{A. Tehamaensis, Gabb.}

A. subtricarinatus, Gabb (not d'Orb.); Vol. 1, p. 60, pl. 10, fig. 4.

A. Tehamaensis, Gabb; Vol. 2, p. 132.

Shasta Group (?), Battle Creek, Tchama County.

\section{A. Chicoensis, Trask.}

A. Chicoensis, Trask; Proc. Cal. Acad., Vol. 1, p. 85, pl. 2, fig. 1. Id., Gabb; Vol. 1, p. 68, pl. 13, fig. 17, $17 a, 17 b$.

Chico Group, Chico Creek, Pence's, Kelly's, Siskiyou Mrountains, San Luis Gonzaga, Cottonwood Creek.

\section{A. Suciannsis, Meek.}

A. complexus var. Suciaensis, Meek; Proc. Phil. Acad., 1861, p. 317.

A. complexus? Gabb ; Vol. 1, p. 69.

A. Suciaensis, Gabb; Vol. 2, p. 133, pl. 21, fig. 11, a, b.

Chico Group, Folsom, near Mount Diablo, and Sucia Island, in the Gulf of Georgia.

\section{A. Vancouverensis, Meek.}

A. Vancouverensis, Meek; Proc. Phil. Acad., 1861, p. 317.

Comax, Vancouver Island. 


\section{A. JUGALIS, Gabb.}

A. jugalis, Gabb; Vol.:2, p. 133, pl. 22, fig. 12, a, b, 13 a.

A. Newberryanus, Gabb (not Meck); pars, Vol. 1, pl. 10, fig. 5 ; fig. $6 b$.

Martinez Group, Martinez, Benicia, Curry's, south of Mount Diablo; Tejon

Group, Martinez, and Clayton.

\section{A. Newberryanus, Meek.}

A. Newberryanus, Meek; Trans. Alb. Inst., Vol. 4, p. 47.

Id., Gabb, pars, Vol. 1, p. 61, pl. 27, fig. 199, a, b, c.

Chico Group, Vancouver Island.

\section{A. Whitneyi, Gabb.}

A. Whitneyi, Gabb; Vol. 2, p. 134, pl. 22, fig. 14, a, b. Shasta Group, Cotton wood Creek.

\section{A. Stoliczkanus, Gabb.}

A. Stoliczkanus, Gabb; Vol. 2, p. 135, pl. 23, fig. 16, 16 a. Shasta Group, Cottonwood Creek.

\section{A. Fraternus, Gabb.}

A. fraternus, Gabb ; Vol. 2, p. 137, pl. 23, fig. 15, a, b. Martinez Group, Benicia.

\section{? A. CooperiI, Gabb.}

? A. Cooperii, Gabb; Vol. 1, p. 69, pl. 14, fig. 23, 23 a. Chico Group,? San Diego.

Hamites, Park. H. (? Ancy loceras) Vancouverensis, Gabb.

Hamites Vancouverensis, Gabb; Vol. 1, p. 70, pl. 13, fig. 18.

Chico Group, Vancouver Island.

HELICOCERAS, d'Orb. ? H. vermicularis, Gabb.

? H. vermicularis, Gabb ; Vol. 1, p. 71, pl. 13, fig. 19, 19 a. Martinez Group, near Martinez. 
H. BreweriI, Gabb.

H. Brewerii, Gabb; Vol. 1, p. 72, pl. 14, fig. 22.

Chico Group, Pence's.

\section{H. DeCuIve, Gabb.}

II. declive, Gabb ; Vol. 1, p. 73, pl. 28, fig. 200, $200 a$.

Chico Group, Pence's Ranch.

TURRILItes, Lam. T. Oregonensis, Gabb.

T. sp. indet., Gabb; Vol. 1, p. 73, pl. 20, fig. 201.

T. Oregonensis, Gabb ; Vol. 2, p. 138.

Chico Group, Jacksonville, Oregon, and Crooked Creek of the Des Chutes, east of the Cascade Range.

ANCYIOCERAS, d'Orb. A. Rémondi, Gabb.

Crioceras (A. ?) Rémondii, Gabb; Vol. 1, p. 75, pl. 14, fig. $24 a$. Ancyloceras sp. ? Gabb; Vol. 1, p. 78, pl. 15, fig. 30.

A. Rémondii, Gabb; Vol. 2, p. 138, pl. 23, fig. 17.

Shasta Group, Cottonwood Creek, Arbuckle's.

\section{A. Percostatus, Gabb.}

Crioceras percostatus, Gabb ; Vol. 1, p. 77, pl. 16, fig. 26; pl. 17, fig. 26 a.

A. percostatus, Gabb; Vol. 2, p. 138, pl. 24, fig. 19.

Shasta Group, Cottonwood Creek, Arbuckle's, and a fragment (of this species ?) from the vicinity of Martinez.

\section{? A. quadratus, Gabb.}

Ptychoceras (? Hamites) quadratus, Gabb; Vol. 1, p. 74, pl. 15, fig. 21; pl. 14, fig. $21 a$.

Chico Group, Pence's Ranch.

\section{? A. Lineatus, Gabb.}

? A. lineatus, Gabb ; Vol. 2, p. 139, pl. 23, fig. 18, $a, b, c$.

Shasta (?) or Chico Group, Cottonwood Creek, and Chico Group near Folsom.

CRIOCERAS, d'Orb. C. LATUs, Gabb.

C. latus, Gabb; Vol. 1, p. 76, pl. 15, fig. $2525 a$; pl. 14, fig. $25 b$. Shasta Group, near Weaverville. 
IIELICANCYLUS, Gabb. H. AQUICOSTATUS, Gabb.

Ptychoceras aquicostatus, Gabb; Vol. 1, p. 74, pl. 13, fig. 20.

H. cqquicostatus, Gabb; Vol. 2, p. 141, pl. 25, tig. 20, a-g.

Shasta Group, Cottonwood Creck, Alderson's Gulch, and Eagle Creek, Shasta County.

DIPTYCHOCERAS, Gabb. D. LAVIS, Gabb.

D. lavis, Gabb; Vol. 2, p. 144, pl. 25, fig. 21, a, b. Shasta Group, Cottonwood Creck.

Baculites, Lam. B. Chicoensis, Trask.

B. Chicoensis, Trask ; Proc. Cal. Acad., 1856, p. 85, pl, 2, fig. 2.

B. Chicoensis, Gabb; Vol. 1, p. 80, pl. 17, fig. 27, $27 a$; pl. 14, fig. 27 b, 29, $29 a$. Chico Group, Chico, Pence's, Cottonwood, Orcstimba, and San Diego; Mar. tincz Group, Martincz.

B. occidentalis, Meek.

B. ovatus, ? Meek; Tr. Alb. Inst., Vol. 4, p. 48.

B. occidcntalis, Meck; loc. cit., P. 49.

Il., Meck; Proc. Phil. Acad., 1861, p. 316.

B. sp. indet., Gabb ; Vol. 1, p. 81, pl. 14, fig. 28 b; pl. 17, fig. $28,28 a$.

Chico Group, Vancouver Island.

B. inornatus, Meek.

B. inornatus, Mcck; Proc. Phil. Acad., 1861, p. 316.

Sucia Island, Gulf of Georgia.

\section{GASTEROPODA.}

TYPHIS, Montf. T. ANTIQuUs, Gabb.

T. antiques, Gabb; Vol. 1, p. 82, pl. 18, fig. 31 .

Tejon Group, Maritnez, Tejon.

FUSUS, Lam. F. Martinez, Gabb.

F. Martinez, Gabb; Vol. 1, p. 82, pl. 18, fig. 32.

Tejon Group, Martinez, Tejon. 
F. MathewsoniI, Gabb.

F. Mathewsonii, Gabb; Vol. 1, p. 83, pl. 18, fig. 33.

Martinez Group, Martinez and Curry's; Tejon Group, Martinez, Clayton and Cochrane's.

$$
\text { F. Averillit, Gabb. }
$$

F. Averillii, Gabb; Vol. 1, p. 83, pl. 18, fig. 34.

Chico Group, Tuscan Springs.

$$
\text { F. Diaboli, Gabb. }
$$

F. Diaboli, Gabb; Vol. 1, p. 84, pl. 18, fig. 35.

Tejon Group, vicinity of Mount Diablo, Tejon.

$$
\text { F. aratus, Gabb. }
$$

F. aratus, Gabb; Vol. 1, p. 84, pl. 28, fig. 202.

Group? (Martinez or Chico) Martinez.

$$
\text { F. Flexuosus, Gabb. }
$$

F. flexuosus, Gabb; Vol. 1, p. 85, pl. 21, fig. 109.

Martinez Group, Martinez.

$$
\text { F. KinciI, Gabb. }
$$

F. Kingii, Gabb; Vol. 1, p. 85, pl. 28, fig. 204.

Chico Group, Cottonwood Creek, Siskiyou County (not the creek of the same name in Shasta County).

\section{F. Californicus, Con., sp.}

F. Californicus, Gabb; Vol. 1, p. 85, pl. 28, fig. $205 a$.

2 Clavatula Californica, Con.; P. R. R. Report, Vol. 5, p. 322, pl, 2, fig. 11. Tejon Group, Clayton, Tejon; beds intermediate between the Tejon and Martinez Groups, Lower Lakc Village, Lake County.

$$
\text { F. tumidus, Gabb. }
$$

F. tumidus, Gabb ; Vol. 2, p. 145, pl. 26, fig. 22. Martinez Group, Martinez.

$$
\text { F. occidentalis, Gabb. }
$$

F. occidentalis, Gabb; Vol. 2, p. 146, pl. 26, fig. 23. Martinez Group, Martinez. 
NePtUnea, Bolt. N. (Tritonofusus) cretacea, Gabb.

Neptunea (Tritonofusus) cretacea, Gabb; Vol. 2, p. 146, pl. 26, fig. 24.

Tejon Group, Martinez.

N. Mucronata, Gabb.

N. mucronata, Gabb; Vol. 2, p. 147, pl. 26, fig. 25.

Martinez Group, Martincz.

? N. supraplicata, Gabb.

2. N. supraplicata, Gabb; Vol. 1, p. 89, pl. 18, fig. 40 .

Tejon Group, Clayton, and San Diego.

? N. Gracilis, Gabb.

N. gracilis, Gabb; Vol. 1, p. 90, pl. 18, fig. 42.

N. Curvirostris, Gabb.

N. curvirostris, Gabb, Vol. 1, p. 88, pl. 18, fig. 37.

Chico Group, Cow Creek.

PAL Fatractus, Gabb. P. CRassus, Gabb.

P. crassus, Gabb; Vol. 2, p. 148, pl. 26, fig. 26.

Shasta(?) Group, Colusa County, near Sulphur Springs.

ERIPACHYA, Gabb. E. Ponderosa, Gabb.

Neptunea ponderosa, Gabb ; Vol. 1, p. 88, pl. 18, fig. 38.

Eripachya ponderosa, Gabb; Vol. 2, p. 149.

Chico Group, Tuscan Springs, Pence's.

E. perforata, Gabb.

Neptunea perforata, Gabb; Vol. 1, p. 89, pl. 13, fig. 39 .

Eripachya perforata, Gabb; Vol. 2, p. 149.

Shasta Group, Cotton wood Creek.

E. HofFManNII, Gabb.

Neptunea Hoffmannii, Gabb; Vol. 1, p. 90, pl. 18, fig. 41 Eripachya Hoffmannii, Gabb; Vol. 2, p. 149.

Shasta Group, Cottonwood Creek. 
PERISSOLAX, Gabb. P. BRevirostris, Gabb.

P. brevirostris, Gabb; Vol. 1, p. 91, pl. 19, fig. 43.

Chico Group, Tuscan Springs, Pence's Ranch; Martinez Group, Martinez; beds intermediate between Martinez and Tejon Group, Lower Lake Village, Lake County.

$$
\text { P. Bhakei, Con., sp. }
$$

Busycon? Blakei, Con.; Pacific R. R.'Rep., Vol. 5, p. 322, pl. 2, fig. 13.

Lovifusus Blakei, Con.; Smithsonian Check List.

Perissolax Blakei, Gabb; Vol. 1, p. 92, pl. 21, fig. 110.

Id., Gabb; vol. 2, p. 149.

Tejon Group, Martinez, Clayton, Coehrane's, and Tcjon.

SURCULA, H. \& A. Ad. S. PReattenuata, Gabb.

S. procattenuata, Gabb; Vol. 2, p. 150, pl. 26, fig. 27.

Tejon Group, San Diego.

S. (Surculites) sinuata, Gabb.

Conus sinuatus, Gabb; Vol. 1, p. 123, pl. 29, fig. 227.

Surcula (Surculites) sinuata, Gabb; Vol. 2, p. 150, pl. 26, fig. 28.

Tejon Group, Tejon.

S. (Surculites) inconspicua, Gabb.

S. (S.) inconspicua, Gabb ; Voḷ. 2, p. 151, pl. 26, fig. 29.

Martinez Group, Martinez.

S. Claytonensis, Gabb.

Turris Claytonensis, Gabb ; Vol. 1, p. 92, pl. 18, fig. 46.

Tejon Group, Clayton, Tejon.

S. raricostata, Gabb.

Turris raricostata, Gabb; Vol. 1, p. 93, pl. 18, fig. 47.

(varicostata, typographical error.)

Surcula id., Con.; Sm. Check List, Eocene, No. 588.

Tejon Group, Clayton.

HETEROTERMA, Gabb. H. TrochoIdea, Gabb.

H. trochoidea, Gabb; Vol. 2, p. 152, pl. 26, fig. 30, 30 a.

Martinez Group, Martinez.

PAL. VOL. II.-29 
BELA, Leach. B. Clathrata, Gabb.

Bela clathrata, Gabb; Vol. 2, p. 152, pl. 26, fig. 31.

Tejon Group, Martinez.

CORDIERA, Rouault. C. Microptygma, Gabb.

C. microptygma, Gabb ; Vol. 1, p. 93, pl. 28, fig. 203.

Tejon Group, Tejon.

C. MITRAEFormis, Gabb.

C. mitrceformis, Gabb; Vol. 2, p. 153, pl. 26, fig. 32.

Shasta Group, Colusa County, near the Sulphur Springs.

TRITONIUM, Link. 'T. HoRnir, Gabb.

Tritonium Hornii, Gabb; Vol. 1, p. 94, pl. 28, fig. 208.

Tejon Group, Tejon and Cochrane's, near Mount Diablo.

T. Paucivaricatum, Gabb.

T. paucivaricatum, Gabb; Vol. 1, p. 95, pl. 28, fig. 209 a.

Tejon Group, Tejon.

T. Whitneyi, Gabb.

T. Whitneyi, Gabb; Vol. 1, p. 96, pl. 28, fig. $210 a$.

Tejon Group, Tejon, San Diego.

T. Californicum, Gabb.

Tritonium Californicum, Gabb; Vol. 2, p. 154, pl. 26, fig. 33.

Tejon Group, Tejon.

Tritonidm, (S. Gen. Trachytriton, Meek). T. (T.) Diegoensis, Gabb.

Tritonium Diegoensis, Gabb ; Vol. 1, p. 95, pl. 18, fig. 44.

Tejon Group, San Diego.

T. (T.) Tejonensis, Gabb.

T. (T.) Tejonensis, Gabb; Vol. 2, p. 154, pl. 26, fig. 34.

Tejon Group, Tejon. 
T. (T.) FUSIFor Me, Gabb.

T. (T.) fusiforme, Gabb; Vol. 2, p. 155.

Figure without name or description, Vol. 1, pl. 18, fig. 45.

Tejon Group, Tejon.

BRACHYSPHINGUS, Gabb. B. LIRATUS, Gabb.

Buccinum liratum, Gabb; Vol. 1, p. 96, pl. 28, fig. 211.

Brachysphingus liratus, Gabb; Vol. 2, p. 156.

Tejon Group, Martinez, Clayton, Marsh's.

B. sinuatus, Gabb.

B. sinuatus, Gabb; Vol. 2, p. 156, pl. 26, fig. 35.

Tejon Group, Martinez.

BUllia, Gray. (S. Gen. Molopophorus, Gabb.) B. (M.) striata, Gabb.

B. (M.) striata, Gabb; Vol. 2, p. 157, pl. 26, fig. 36.

Tejon Group, Tejon.

NASSA, Lam. N. CRetacea, Gabb.

N. eretacea, Gabb; Vol. 1, p. 97, pl. 18, fig. 49.

Tejon Group, Martinez, Tejon, and ten miles west of Griswold's.

N. ANTIQUata, Gabb.

N. antiquata, Gabb; Vol. 1, p. 97, pl. 18, fig. 50.

Tejon Group, Martinez.

HAYDENIA, Gabb. H. IMPRESSA, Gabb.

Haydenia impressa, Gabb ; Vol. 1, p. 98, pl. 18, fig. 51.

Chico Group, Tuscan Springs, and Pence's.

PSEUdoliva, Swains. P. LIneata, Gabb.

P. lineata, Gabb; Vol. 1, p. 99, pl. 18, fig. 52.

Tejon Group, Martinez. 
P. volut жformis, Gabb.

P. volutaformis, Gabb; Vol. 1, p. 99, pl. 28, fig. 212.

Tejon Group, Tejon.

OIIVELla, Swains. O. Mathewsonir, Gabb.

O. Mathewsonii, Gabb; Vol. 1, p. 100, pl. 18, fig. 53.

Tejon Group, Martinez, Tejon, Clayton, and Griswold's.

AHCILLARIA, Lam. A. elongata, Gabb.

A. elongata, Gabb ; Vol. 1, p. 100, pl. 18, fig. 54.

Tejon Group, Clayton, Cochrane's, and San Diego.

Fasciolaria, Lam. ? F. Leviuscula, Gabb.

? F. laviuscula, Gabb; Vol. 1, p. 100, pl. 18, fig. 55 .

Beds intermediate between the Martinez and Tejon Groups at Clayton, and near Lower Lake Village.

F. sinuata, Gabb.

F. sinuata, Gabb; Vol. 1, p. 101, pl. 28, fig. $213 a$.

Tejon Group, Tejon, San Diego.

$$
\text { F. Io, Gabb. }
$$

? F. Io, Gabb; Vol. 1, p. 101, pl. 28, fig. 214.

Tejon Group, Tejon.

TURBINELla, Lam. T. CRAssitesta, Gabb.

Turbinella crassitesta, Gabb ; Vol. 2, p. 157, pl. 26, fig. 37.

Martinez Group, Martinez.

VOLUTILITHES, Swains. V. NAVArroensis, Shum.?

V. Navarroensis, Shum.; Proc. Bost. Soc. N. Hist., 1861, Vol. 8, p. 192.

Id., ? Gabb; Vol. 1, p. 102, pl. 19, fig. 56.

Chico Group, Tusean Springs, Pence's, Jacksonville, Oregon, Siskiyou Mountains, Chico Creek, Cow Creek. 
MitRA, Lam. M. CRetacea, Gabb.

Mitra cretacea, Gabb; Vol. 1, p. 103, pl. 28, fig. 215.

Id., Gabb ; Vol. 2, p. 158.

Tejon Group, Martinez.

WHITNEYA, Gabb. W. FICUS, Gabb.

Whitneya ficus, Gabb; Vol. 1, p. 104, pl. 28, fig. 216.

Tejon Group, Tejon.

FicUs, Bolt. F. mamiliatus, Gabb.

F. mamillatus, Gabb; Vol. 1, p. 211, pl. 32, fig. 276.

Tejon Group, Tejon.

FICOPSIS, Con. F. Rémondi, Gabb.

Fusus (Hemifusus) Rémondii, Gabb; Vol. 1, p. 87, pl. 18, fig 36.

Tejon Group, Martinez, Clayton, Griswold's, Tejon.

$$
\text { F. HornII, Gabb. }
$$

Fusus (Hemifusus) Hornii, Gabb; Vol. 1, p. 86, pl. 28, fig. 206.

Tejon Group, Tejon.

$$
\text { F. Cooperit, Gabb. }
$$

Fusus (Hemifusus) Cooperii, Gabb; Vol. 1, p. 86, pl. 28, fig. 207.

Tejon Group, Clayton, and San Diego.

UROSYCA, Gabb. U. CAUdata, Gabb

U. caudata, Gabb; Vol. 2, p. 159, pl. 27, fig. 38.

Martinez Group, Martinez

SYCODES, Gabb. S. CYPR EOIDES, Gabb.

? Ficus cyprooides, Gabb ; Vol. 1, p. 105, pl. 19, fig. 58.

Sycodes cyprcooides, Gabb ; Vol. 2, p. 160.

Chico Group, Tuscan Springs, Texas Flat.

Natica, Brug. N. Uvasana, Gabb.

Natica Uvasana, Gabb; Vol. 1, p. 212, pl. 32, fig. 277.

Tejon Group, Tejon. 
LUNATIA, Lam. L. avellana, Gabb.

L. avellana, Gabb; Vol. 1, p. 105, pl. 19, fig. 60 .

Shasta Group, Cottonwood Creek.

\section{Shumardiana, Gabb.}

L. Shumardiana, Gabb; Vol. 1, p. 106, pl. 19, fig. 61 .

Martinez Group, Martinez; intermediate beds forming transition to the Tejon

Group, Lower Lake, Lake County.

\section{HornII, Gabb.}

L. Hornii, Gabb; Vol. 1, p. 106, pl. 29, fig. 217.

Tejon Group, Tejon.

\section{NUCIFormis, Gabb.}

L. nuciformis, Gabo; Vol. 1, p. 107, pl. 28, fig. 218.

Tejon Group, Clayton, Tejon, and (?) San Diego.

GYRoDes, Con. G. Conradiana, Gabb.

Lunatia (? Gyrodes) Conradiana, Gabb ; Vol. 1, p. 107, pl. 29, fig. 219.

Chieo Group, Paeheeo's Pass.

\section{G. Expansa, Gabb.}

Gyrodes expansa, Gabb; Vol. 1, p. 108, pl. 19, fig. 62, $a, b, c$.

Chieo Group, Penee's, Cottonwood Creek, Tusean Springs, Texas Flat, Siskiyou Mountairs, and Jaeksonville; Martinez Group, Martinez; and in the transition beds, Lower Lake.

NEVERITA, Risso. N. SEcta, Gabb.

N. secta, Gabb; Vol. 1, p. 108, pl. 29, fig. $220 a$.

Tejon Group, Tejon.

N. globosa, Gabb.

Neverita globosa, Gabb; Vol. 2, p. 161, pl. 27, fig. 39.

Tejon Group, Griswold's, and New Idria. 
NATICINA, Gray. N. obliqua, Gabb.

N. obliqua, Gabb; Vol. 1, p. 109, pl. 21, fig. 112.

Catinus obliquus, Con.; Sm. Check List, No. 470.

Tejon Group, Martinez, Tejon.

AMAUROPSIS, Mörch. A. oviformis, Gabb.

A. oviformis, Gabb; Vol. 1, p. 109, pl. 19, fig. 63.

Chico Group, Tuscan Springs.

EUspira, Agas. E. Alveata, Con., sp.

Natica alveata, Con.; Pacific R. R. Rep., Vol. 5, p. 321, pl. 2, fig. 8.

Amauropsis alveata, Gabb; Vol. 1, p. 110, pl. 19, fig. 59; pl. 21, fig. 111.

Ampullina alveata, Con.; Sm. Check List, No. 459.

Chico Group, Curry's, south of Mount Diablo; Martinez Group, Martinez; intermediate beds, Lower Lake; Tejon Group, Martinez, Clayton, Griswold's, Tejon, and San Diego.

AMPULlina. A. striata, Gabb.

A. striata, Gabb ; Vol. 2, p. 161, pl. 27, fig. 40.

Martinez Group, Martinez.

MORI0, Montf. M. (Sconsia) tuberculatus, Gabb.

M. (Sconsia) tuberculatus, Gabb ; Vol. 1, p. 104, pl. 19, fig. 57.

Tejon Group, Martinez, Clayton, Griswold's, Tejon, and San Diego.

SCALARIA, Lam. S. (Opalia) Mathewsonit, Gabb.

Scalaria (Opalia) Mathewsonii, Gabb; Vol. 1, p. 212, pl. 32, fig. 278.

Tejon Group, Martinez.

TEREBRA, Brug. T. Californica, Gabb.

T. Californica, Gabb; Vol. 2, p. 162, pl. 27, fig. 41.

Tejon Group, Martinez.

Chemnitzia, d'Orb. C. plandlata, Gabb.

C. Spillmani, Gabb (not Con.); Vol. 1, p. 115, pl. 19, fig. 70.

'C. planulata, Gabb; Vol. 2, p. 162.

Chico Group, Pence's. 
INIS0, Risso. N. Polita, Gabb.

Niso polita, Gabb; Vol. 1, p. 116, pl. 21, fig. 113.

Tejon Group, Martinez, and Tejon.

CERITHIOPSIS, F. \& H. C. alternata, Gabb.

Cerithiopsis alternata, Gabb; Vol. 1, p. 116, pl. 21, fig. 114, 114 a.

Tejon Group, Martinez, and east of Mount Diablo.

\section{ARCHItectonica, Bolt. A. Veatchi, Gabb.}

A. Veatchii, Gabb; Vol. 1, p. 116, pl. 19, fig. 71.

Chico Group, Tuscan Springs.

A. cognata, Gabb.

A. cognata, Gabb; Vol. 1, p. 117, pl. 20, fig. 72, $a, b, c, d, e$ Tejon Group, Martinez, Clayton, Tejon

\section{A. Hornil, Gabb.}

A. Hornii, Gabb; Vol. 1, p. 117, pl. 29, fig. 224, $a, b$.

Tejon Group, Tejon.

A. inornata, Gabb.

A. inornata, Gabb; Vol. 1, p. 118, pl. 20, fig. 73.

Chico Group, Tuscan Springs; Martinez Group, Martinez.

Discohelix, Dkr. D. Leana, Gabb.

- Discohelix Leana, Gabb; Vol. 1, p. 119, pl. 20, fig. 75.

Chico Group, Texas Flat, Placer County.

STRAPAROLLUS, Montf. S. Paucivolvus, Gabb.

S. paucivolvus, Gabb; Vol. 1, p. 120, pl. 20, fig. 76 .

Chico Group, Texas Flat.

S. LeNs, Gabb.

Straparollus lens, Gabb; Vol. 1, p. 120, pl. 20, fig. 77, a-d. Chico Group, Texas Flat. 
conUs, Linn. C. Rémondi, Gabb.

Conus Rémondii, Gabb; Vol 1, p. 122, pl. 20, fig. 79.

Volutilithes Californica, Con.; Pacific R. R. Rep., Vol. 5, p. 322, pl. 2, fig. 9.

Tejon Group, Martinez, Clayton, Cochrane's, Tejon, San Dicgo.

$$
\text { C. Hornir, Gabb. }
$$

C. Hornii, Gabb ; Vol. 1, p. 122, pl. 29, fig. 226.

Tejon Group, Tejon.

PUGNellus, Con. P. hamulus, Gabb.

P. hamulus, Gabb; Vol. 1, p. 124, pl. 20, fig. 81 ; pl. 18, fig. 48.

Pugnellus hamulus, Gabb; Vol. 2, p. 162, pl. 27, fig. 42, 42 a.

Martinez Group, Martinez.

$$
\text { P. (Gymnarus) manubriatus, Gabb. }
$$

Pugnellus manubriatus, Gabb; Vol. 1, p. 125, pl. 29, fig. 229 a.

P. (Gymnarus) manubriatus, Gabb ; Amer. Jour. Conch., 1868, p. 139.

Id., Gabb; Vol. 2, p. 163.

Chico Group, Cottonwood Creek, Siskiyou County.

RIMELIA, Agas. R. canalifera, Gabb.

Rostellaria (Rimella) canalifera, Vol. 1, p. 123, pl. 29, fig. 228.

Rimella canalifera, Gabb; Proc. Cal. A. N. S., 1866.

Tejon Group, Martinez and Tejon.

\section{R. Simplex, Gabb.}

Rostellaria (Rimella) simplex, Gabb; Vol. 1, p. 124, pl. 20, fig. 80.

Rimella simplex, Gabb; Proc. Cal. A. N. S., 1866.

Tejon Group, Clayton, San Diego.

CYPREA, Linn. C. (Luponia) Bayerquei, Gabb.

, Cyproa Bayerquei, Gabb; Vol. 1, p. 129, wood cuts.

c. (L.) Bayerquei, Gabb; Vol. 2, p. 163, pl. 27, fig. 43, $a, b, c$.

'Tejon Group, Martinez, Clayton, Tejon.

$$
\text { C. (Epona) Matuewsonit, Gabb. }
$$

C. (E.) Mathewsonii, Gabb; Vol. 2, p. 164, pl. 27, fig. 44, $a, b$.

Tejon Group, Martinez.

PAL. VOL. II. -30 . 
ANCHURA, Con. A. Falciformis, Gabb.

Aporrhais,falciformis, Gabb ; Vol. 1, p. 127, pl. 20, fig. 83.

Anchura falciformis, Gabb; Amer. Jour. Conch., 1868, p. 145

A. falciformis, Gabb; Vol. 2, p. 165.

Chico Group, Tuscan Springs, Chico, Pence's, Texas Flat.

A. angulata, Gabb.

Aporrhais angulata, Gabb; Vol. 1, p. 128, pl. 20, fig. 84.

Martinez, and (?) Huling Creck, Shasta County.

\section{A. Californica, Gabb.}

Aparrhais Californica, Gabb; Vol. 1, p. 128, pl. 29, fig. 230, a, b.

Chico Group, Orestimba, Martinez, Puer to Cañon, and Siskiyou Mountains.

A. Exilis, Gabb.

Aporrhais exilis, Gabb; Vol. 1, p. 129, pl. 29, fig. 231.

Martinez (?) Group, Martinez.

A. transversa, Gabb.

A. transversa, Gabb; Vol. 2, p. 165, pl. 27, fig. 45.

Martinez Group, Martinez.

\section{? A. Carinifera, Gabb.}

2. A. carinifera, Gabb; Vol. 2, p. 166, pl. 28, fig. 46. Martinez Group, Martinez.

helicadlaX, Gabb. H. Bicarinata, Gabb.

II. bicarinata, Gabb; Vol. 2, p. 166, pl. 27, fig. 47. Shasta Group, Cottonwood Crcek.

$$
\text { H. costata, Gabb. }
$$

H. costata, Gabb ; Vol. 2, p. 167, pl. 28, fig. 48. Martinez Group, Martinez.

TESSAROLAX, Gabb. T. Distorta, Gabb.

T. distorta, Gabb; Vol. 1, p. 126, pl. 20, fig. 82, a, b.

Chico Group, Tuscan Springs. 
IOXOTREMA, Gabb. L. TURRITA, Gabb.

Loxotrema turrita, Gabb ; Amer. Jour. Conchology, 1868, p. 147.

$L$ turrita, Gabb; Vol. 2, p. 168, pl. 28, fig. 49.

Tejon Group, near Griswold's.

Potamides, Brogn. P. diadema, Gabb.

Potamides diadema, Gabb ; Vol. 1, p. 130, pl. 20, fig. 85.

Shasta Group, Cottonwood Creek.

$$
\text { P. tenuis, Gabb. }
$$

Potamides tenuis, Gabb ; Vol. 1, p. 130, pl. 20, fig. 86. Chico Group, Pence's.

IITTORINA, Ferr. L. compacta, Gabb.

? Littorina compacta, Gabb; Vol. 1, p. 131, pl. 20, fig. 89.

Chico Group, Texas Flat, Placer County.

ATRESIUS, Gabb. A. LIRAtUS, Gabb.

A. liratus, Gabb ; Vol. 2, p. 169, pl. 28, fig. 50.

Shasta Group, Colusa County.

TURRITELLA, Lam. T. infralineata, Gabb.

T. infralineata, Gabb; Vol. 1, p. 131, pl. 20, fig. 87.

Chico Group, Cottonwood Creek, and Orestimba.

T. Infragranulata, Gabb.

T. infragranulata, Gabb ; Vol. 1, p. 212, pl. 32, fig. 279.

Martinez (?) Group, Martinez.

T. seriatim-granulata, Roem.

T. seriatim-granulata, Roem; Kreid. Tex., p. 39, pl. 4, fig. 12.

T. planilateralis, Con.; Emory's Rep., Mex. Boundary, p. 158, pl. 14, fig 1.

T. irrorata, Con.; Proc. Phil. Acad., 1855, p. 268.

Chico Group, Tuscan Springs, Cottonwood Creek, Shasta County, and Cot. tonwood Creek, Siskiyou County. Also found in Texas, and at Arivechi, Sonora. 
T. Veatchir, Gabb.

T. Veatehii, Gabb; Vol. 1, p 133, pl. 20, fig. 90. Chieo Group, Tusean Springs.

\section{T. Chiconasis, Gabb.}

T. Chicocnsis, Gabb ; Vol. 1, p. 133, pl. 21, fig. 91.

Chieo Group, Chieo Creek.

\section{T. Uvasana, Con.}

Turritella Uvasana, Con.; Paeifie R. R. Rep., Vol. 5, p. 321, pl. 2, fig. 12. Id., Gabb ; Vol. 1, p. 134, pl 21, fig. 92.

Tejon Group, Martinez, Tejon, Clayton, and Griswold's.

\section{T. SAFFordi, Gabb.}

T. Saffordii, Gabb; Jour. Phil. Aead., 2 Ser., Vol. 4, p. 392, pl. 68, fig. 12. Ill., Gabb; Vol. 1, p. 135, pl. 21, fig. 93.

Compare 'T. (Torcula) dispassa, Stol.; Pal. Ind., Gast., pl. 16, figs. 13, 14. Martinez Group, Martinez, and near Suisun; beds between Martinez and Tejon Groups, near Lower Lake Village. Also in the Ripley Group in Tennessee and New Jersey (and India?).

T. robusta, Gabb.

Turritella robustu, Gabb; Vol. 1, p. 135, pl. 21, fig. 94.

Chieo Group, Tusean Springs.

T. Martinezensis, Gabb.

T. Martinezensis, Gabb; Vol. 2, p. 169, pl. 28, fig. 51.

Beds intermediate between the Martinez and Tejon Groups, Martinez.

GALERUS, Humph. G. excentricus, Gabb.

G. excentricus, Gabb; Vol. 1, p. 136, pl. 20, fig. 95; pl. 29, fig. 232.

Tejon Group, Martinez. Clayton, San Diego, Tejon, \&e.

SPIROCRYPTA, Gabb. S. PILEUn, Gabb.

Crypta (Spirocrypta) pilcum, Gabb ; Vol. 1, p. 137, pl. 29, fig. 233, a, b. Tejon Group, Tejon, New Idria.

NERITA, Linn. N. DEFormis, Gabb.

N. deformis, Gabb; Vol. 1, p. 137, pl. 21, fig. 96.

Sbasta Group, Cottonwond Creek, Shasta County. 
N. cuneata, Gabb.

N. cuneata, Gabb; Vol. 1, p. 137, pl. 21, fig. 97. Chico Group, Tuscan Springs.

N. ('Themiostyla) triangulata, Gabb.

$N$. (T.) triangulata, Gabb; Vol. 2, p. 170, pl. 28, fig. 62 a.

Tejon Group, New Idria.

LYSIS, Gabb. L. DUPLICosta, Gabb.

Lysis duplicosta, Gabb; Vol. 1, p. 138, pl. 21, fig. 98.

Chico Group, Texas Flat.

Calliostoma, Swains. C. radiatum, Gabb.

C. radiatum, Gabb ; Vol. 2, p. 170, pl. 28, fig. 53.

Chico Group, Texas Flat.

ATAPHRUS, Gabb. A. CRAssus, Gabb.

Ataphrus crassus, Gabb; Vol. 2, p. 171, pl. 28, fig. 51.

Martinez (?) Group, Martinez.

angaria, Bolt. A. ornatissima, Gabb.

A. ornatissima, Gabb ; Vol. 1, p. 121, pl. 20, fig. 78 .

Chico Group, Texas Flat, and Tuscan Springs.

Margaritella, Meek and Hayden. M. crenulata, Gabb.

M. crenulata, Gabb; Vol. 1, p. 118, pl. 20, fig. 74.

Tejon Group, San Diego.

M. gLobosa, Gabb.

M. globosa, Gabb ; Vol. 1, p. 119, pl. 29, fig. 225.

Chico (?) Group, Benicia.

$$
\text { M. ANGUlate, Gabb. }
$$

M. angulata, Gabb; Vol. 2, p. 172, pl. 28, fig. 65.

Chico Group, Martinez. 
Dentalium, Limn. D. Nanaimoensis, Meek.

D. Nanaimoensis, Meek; Tr. Alb. Inst., 1854, p. 44.

Chico Group, Nanaimo, Vaneouver Island.

$$
\text { D. Cooperit, Gabb. }
$$

D. Cooperii, Gabb ; Vol. 1, p. 139, pl. 21, fig. 100.

Chieo Group, Curry's, south side of Mount Diablo; Martinez Group, Martinez; Tejon Group, Martinez, Tejon, and San Diego.

D. stramineum, Gabb.

D. stramineum, Gabb; Vol. 1, p. 139, pl. 21, fig. 101.

Assoeiated with the preeding at eaeh of the above loealities.

GADUS, Rang. G. pusiluus, Gabb.

Dentalium (Ditrupa ?) pusillum, Gabb; Vol. 1, p. 139, pl. 21, fig. 99.

Tejon Group, Martinez, Tejon.

EMARGINUIA, Lam. E. RAdiata, Gabb.

E. radiata, Gabb; Vol. 1, p. 140, pl. 21, fig. $102 a$. Chieo Group, Texas Flat.

PATElla, Linn. P. Traskit, Gabb.

P. Traskii, Gabb; Vol. 1, p. 140, pl. 21, tig. 103.

Chieo Group, Texas Flat.

ACMEA, Esch. ?A. Tejonensis, Gabb.

? A. Tejonensis, Gabb; Vol. 2, p. 172, pl. 28, fig. 56 .

HELCION, Montf. ? H. circularis, Gabb.

II. circularis, Gabb; Vol. 1, p. 141, pl. 29, fig. $234 a$. Martinez Group, Martinez.

H. ріснотома, Gabb.

H. dichotoma, Gabb ; Vol. 1, p. 141, pl. 21, fig. 104.

Chieo Group, Texas Flat. 
ANISOMYON, M. \& H. A. MeEKri, Gabb.

A. Meekii, Gabb ; Vol. 1, p. 142, pl. 21, fig. 105.

Shasta Group, Cottonwood Crcek.

CINULIA, Gray. C. obliqua, Gabb.

Cinulia obliqua, Gabb; Vol. 1, p. 111, pl. 19, fig. 64, a, b, c.

Chico Group, Tuscan Springs, Martinez, Pcnce's, Chico Creek, Texas Flat, Cottonwood Creck (Shasta County), and Cottonwood Creek (Siskiyou County).

\section{Mathewsonir, Gabb.}

C. Mathewsonii, Gabb; Vol. 1, p. 111, pl.19, fig. 65.

Chico Group, Martinez, Orestimba.

RINGICULA, Desh. R. varia, Gabb.

Ringicula varia, Gabb; Vol. 1, p. 112, pl. 29, fig. 222, a, b.

Chico Group, Cow Creek, Shasta County.

RINGINELLA, d'Orb. R. Polita, Gabb.

R. polita, Gabb ; Vol. 2, p. 174, pl. 28, fig. 60 .

Shasta Group, Colusa County.

$$
\text { R. PINGuis, Gabb. }
$$

Cinulia pinguis, Gabb ; Vol. 1, p. 112, pl. 29, fig. 221, a, ๖.

? Martinez Group, Martinez.

NERINEA, Defr. N. DIspaR, Gabb.

Nerinea dispar, Gabb; Vol. 1, p. 113, pl. 19, fig. 66 a.

Shasta Group, Cottonwood Creek.

ACT EONINA, d'Orb. A. PUPOIDEs, Gabb.

2 A. pupoides, Gabb; Vol. 1, p. 113, pl. 19, fig. 67.

A. pupoides, Gabb; Vol. 2, p. 173, pl. 28, fig. 57.

Shasta Group, Cottonwood Crcek.

\section{A. Californica, Gabb.}

A. Californica, Gabb; Vol. 1, p. 114, pl. 19, fig. 68.

Chico Group, (?) Martinez, Benicia, and near Yreka. 
ACT EEONELLA, d'Orb. A. OVIFORMIS, Gabb.

A. oviformis, Gabb; Vol. 2, p. 173, pl. 28, fig. 58.

Chieo Group, (?) Cottonwood Creek.

ACT EE0N, Montf. A. IMPRessus, Gabb.

A. impressus, Gabb ; Vol. 1, p. 142, pl. 21, fig. 106.

Shasta Group, Cotton wood Creek.

LIOCIUM, Gabb. L. PUnCTATUM, Gabb.

Liocium punctatum, Gabb; Vol. 1, p. 174, pl. 28, fig. 59.

Shasta (?) Group, Colusa County.

GLOBICONCHA, d’Orb. G. (Pinasianella?) Rémondi, Gabb.

G. (Phasianella ?) Rêmondii, Gabb ; Vol. 1, p. 114, pl. 19, fig. 69.

Chico (?) Group, Benieia.

CYLINDRITES, M. \& L. C. BREVIS, Gabb.

Cylindritcs brevis, Gabb ; Vol. 1, p. 115, pl. 29, fig. 223.

Martinez Group, Martinez.

BULLA, Brng. B. Hornit, Gabb.

Bulla Hornii, Gabb ; Vol. 1, p. 143, pl. 29, fig. 235.

Tejon Group, Tejon.

CYLIChNA, Lovén. C. costata, Gabb.

Cylichna costata, Gabb ; Vol. 1, p. 143, pl. 21, fig. 107.

Chico Group, Texas Flat, Penee's, Martinez; Martinez Group, Martinez; Tejon Group, Martinez, Clayton, Cochrane's, Tejon, and San Diego.

Megistostoma, Gabb. M. Striatum, Gabb.

Megistostoma striatum, Gabb; Vol. 1, p. 144, pl. 21, fig. 108 a.

Tejon Group, Martinez. 


\section{ACEPHALA.}

MARTESIA, Leach. M. clausa, Gabb.

Martesia clausa, Gabb; Vol. 1, p. 145, pl. 22, fig. 115.

Chico Group; Pcnce's, Texas Flat, Tuscan Springs; Martinez Group, Martinez; Tejon Gioup, Martinez, Tejon, and ten miles west of Griswold's.

TURNUS, Gabb. T. Plenus, Gabb.

Turnus plenus, Gabb; Vol. 1, p. 146, pl. 22, fig. 116.

Shasta Group, Cottonwood Creek; (? Chico Group, Pacheco's Pass).

SOLEN, Linn. S. Parallelus, Gabb.

Solen parallelus, Gabb; Vol. 1, p. 146, pl. 22, fig. 117.

Plectosolen parallelus, Con.; Sm. Check List, No. 241.

Tejon Group, Martinez, Marsh's and Tejon.

S. (Hypogella) cuneatus, Gabb.

S. (Hypogella) cuneatus, Gabb; Vol. 2, p. 175, pl. 29 , fig. 61. Martinez Group, Martinez.

S. (Hypogelia) Diegoensis, Gabb.

S. (Solena) Diegoensis, Gubb; Vol. 1, p. 213, pl. 32, fig. 280.

Plectosolen Diegoensis, Con.; Sm. Check List, No. 240.

S. (Hypogella) Diegoensis, Gabb; Vol. 2, p. 176.

Tejon Group, San Diego.

PHARELIA, Gray. P. Alta, Gabb.

Pharella alta, Gabb; Vol. 1, p. 147, pl. 22, fig. 118.

Martinez Group, Martinez.

SILIQUA, Mublf. S. Oregonensis, Gabb.

Siliqua Oregonensis, Gabb; Vol. 1, p. 147, pl. 29, fig. 237.

Chico Group, Siskiyou Mountains.

CORBULA, Brug. ? C. PRimorsa, Gabb.

2 C. primorsa, Gabb ; Vol. 1, p. 148, pl. 22, fig. 120.

Tejon Group, Corral Hollow.

PAL. VOL. II.-31 
C. Traskit, Gabb.

Corbula Traskii,, Gabb; Vol. 1, p. 149, pl. 22, fig. 121 a.

Chieo Group, Texas Flat, Tusean Springs, and Penee's Raneh.

C. cultriformis, Gabb.

Corbula cultriformis, Gabb; Vol. 1, p. 149, pl. 22, fig. 122.

Martinez Group, Martinez.

\section{HorniI, Gabb}

Corhula Hornii, Gabb; Vol. 1, p. 149, pl. 29, fig. 238.

Corbula Hornii, Gabb; Vol. 2, p. 176, pl. 29, fig. 62, $a, b$.

Tejon Group, Tejon.

$$
\text { C. PARILIS, Gabb. }
$$

Corbula parilis, Gabb; Vol. 1, p. 150, pl. 29, fig. 239 a.

Tejon Group, Martinez, Clayton, Cochrane's, Marsh's, Griswold's, and San Diego.

$$
\text { C. ALæFORMIS, Gabb. }
$$

Corbula alceformis, Gabb; Vol. 2, p. 177, pl. 29, fig. 63.

Beds intermediate between the Martinez and Tejon Groups, Lower Lake, Lake County.

anatina, Lam. A. Tryoniana, Gabb.

Anatina Tryoniana, Gabb; Vol. 1, p. 150, pl. 29, fig. 240.

Chico Group, Martinez.

A. inequilateralis, Gabb.

Anatina inequilateralis, Gabb; Vol. 1, p. 151, pl. 29, fig. 241

Chieo Group, Siskiyou Mountains.

? A. Lata, Gabb.

9. Anatina lata, Gabb; Vol. 1, p. 151, pl. 22, fig. 126.

Chico Group, Penee's.

A. quadrata, Gabb.

Anatina quadrata, Gabb; Vol. 2, p. 177, pl. 29, fig. 64.

Chieo Group, Nanaimo, Vaneouver Island. 
THRACIA, Leach. ? T. occidentalis, Meck.

? Thracia occidentalis, Meek; Trans. Alb. Inst., Vol. 4, p. 43.

Chico Group, Nanaimo, Vancouver Island.

\section{? T. SUbtruncata, Meek.}

? Thracia subtruncata, Meek; loc. cit., p. 44.

With the preceding.

PHOLAdOMYa, Sowb. P. subelongata, Meek.

Pholadomya subelongata, Meek; Tr. Alb. Inst., Vol 4, p. 42. Chico Group, Nanaimo, Vancouver Island.

\section{P. Brewerri, Gabb.}

Pholadomya Brewerii, Gabb; Vol. 1, p. 152, pl. 22, fig. 123.

Chico Group, Pence's Ranch.

$$
\text { P. Nasuta, Gabb. }
$$

Pholadomya nasuta, Gabb ; Vol. 1, p. 152, pl. 30, fig. 124. Martinez Group, Martinez.

\section{P. Oregonensis, Gabb.}

Pholadomya Oregonensis, Gabb; Vol. 2, p. 178, pl. 29, fig. 65.

Chico Group, Siskiyou Mountains.

GONIOMYA, Agas. G. Borealis, Meek.

Pholadomya (Goniomya) borealis, Meek ; Tr. Alb. Inst., Vol. 4, p. 41.

Chico Group, Nanaimo, Vancouver Island.

PLeUromya, Agas. P. Papyracea, Gabb.

Pleuromya papyracea, Gabb; Vol. 2, p. 178, pl. 29, fig. 66.

Shasta Group, Cotton wood Creek.

ARCOMYA, Agas. A. undulata, Gabb.

Arcomya undulata, Gabb; Vol. 2, p. 179, pl. 29, fig. 67.

Chico or Shasta Group, Indian Creek, Butte County. 
HOHOMYA, Agas. H. concentrica, Gabb.

Panopcea concentrica, Gabb; Vol. 1, p. 148, pl. 22, fig. 119.

Homomya concentrica, Gabb; Vol. 2, p. 179.

Chico Group, Tuscan Springs, Cottonwood Creek, and Martinez.

NEARA, Gray. N. DOLABRAFormis, Gabb.

Neara dolabroformis, Gabb ; Vol. 1, p. 153, pl. 22, fig. 125.

Tejon Group, Martinez.

MACTRA, Linn. ? M. tenuissima, Gabb.

2. Mactra tcnuissima, Gabb; Vol. 1, p. 179, pl. 29, fig. 68.

Martinez Group, Martinez.

\section{Gibbsana, Meek.}

Mactra Gibbsana, Meek ; Proc. Phil. Acad., 1861, p. 315.

Cret. ?, 1sland in Gulf of Georgia.

CYHBOPHORA, Gabb. C. Ashburnerit, Gabb.

Mactra Ashburnerii, Gabb; Vol. 1, p. 153, pl. 22, fig. 127.

Cymbophoru Ashburnerii, Gabb; Vol. 2, p. 181, pl. 29, fig. 69.

Chico Group, Pence's, Chico Creek, Texas Flat, Martinez, Iuscan Springs, Orestimba, Benicia, and San Luis Gonzaga; Martinez Group, Martinez and Benicia; Tejon Group, Martinez, Marsh's, Clayton, Griswold's, and Tejon.

LUTRARIA, Lam. L. truncata, Gabb.

Lutraria truncata, Gabb; Vol. 1, p. 154, pl. 22, fig. 128.

Chico Group, l'ence's and Chico Creek.

ASAPhis, Modeer. A. undulata, Gabb.

Asaphis undulata, Gabb; Vol. 1, p. 154, pl. 22, fig. 129.

Chico Group, Texas Flat.

A. multicostata, Gabb.

Asaphis multicostata, Gabb; Vol. 2, p. 181, pl. 29, fig. 70.

Chico Group, Crooked River of Des Chutes, Eastern Oregon. 
GARI, Schum. G. TEXTA, Gabb.

2 Gari tcxta, Gabb ; Vol. 1, p. 155, pl. 22, fig. 130.

Tejon Group, Martinez.

TELLINA, Linn. T. LONGA, Gabb.

Tellina longa, Gabb ; Vol. 1, p 155, pl. 22, fig. 131.

Tejon Group, Martinez, Marsh's, Clayton, Tejon.

\section{T. Rémondit, Gabb.}

Tellina Rêmondii, Gabb ; Vol. 1, p. 156, pl. 22, tig. 132. Id., Gabb ; Vol. 2, p. 182, pl. 29, fig. 71.

Tejon Group, Martinez, Clayton, Cochrane's, Tejon.

\section{T. Hofmmanniana, Gabb.}

Tellina Hoffmanniana, Gabb; Vol. 1, p. 156, pl. 22, fig. $133 a$.

Tellina Hoffmanniana, Gabb; Vol. 2, p. 182, pl. 30, fig. 72.

Chico Group, Pence's ; Martinez Group, Martinez; Tejon Group, Griswold's, and Martinez.

T. honilifera, Gabb.

Tellina monilifera, Gabb; Vol. 1, p. 157, pl. 22, fig. $134 a$. Chico Group, Texas Flat.

T. ooIdes, Gabb.

Tellina ooides, Gabb; Vol. 1, p. 157, pl. 22, fig. $135 a$. Chico Group, Martinez, Pcnce's.

T. Mathewsonit, Gabb.

Tellina Mathewsonii, Gabb ; Vol. 1, p. 158, pl. 23, fig. 136. Chico Group, Martinez.

T. Decurtata, Gabb.

Tellina decurtata, Gabb; Vol. 1, p. 158, pl. 23, fig. 137. Chico Group, Pence's.

? T. QUadrata, Gabb.

? Tellina quadrata, Gabb; Vol. 1, p. 159, pl. 23, fig. 138.

Chico Group, Tuscan Springs. 
T. Ashburnerit, Gabb.

Tellina Ashburnerii, Gabb; Vol. 1, p. 159, pl. 23, fig. 139.

Chieo Group, Pence's.

T. PARILIS, Gabb.

Tellina parilis, Gabb; Vol. 1, p. 160, pl. 30, fig. 243.

Chico Group, Martinez.

\section{T. Hornil, Gabb.}

Tellina Hornii, Gabb; Vol. 1, p. 160, pl. 30, fig. 244

Tejon Group, Tejon, Clayton.

T. Californica, Gabb.

Tellina Californica, Gabb; Vol. 1, p. 161, pl. 30, fig. 245.

Tejon Group, Marslı's, Tejon.

\section{RQUALIS, Gabb.}

Tellina aqualis, Gabb; Vol. 2, p. 182, pl. 29, fig. 73.

Martinez Group, Martinez.

$$
\text { ? T. undulifera, Gabb. }
$$

? Tellina undulifera, Gabb; Vol. 2, p. 183, pl. 30, fig. 74.

Martinez Group, Martinez.

T. (? Sanguinolaria) Whitneyi, Gabb.

Tellina (? Sanguinolaria) Whitneyi, Gabb; Vol. 1, p. 160, pl. 30, fig. 242. Chieo Group, Jaeksonville, Oregon.

DONAX, Linn. D. IATUS, Gabb.

Donax latus, Gabb; Vol. 2, p. 183, pl. 30, fig. 75.

Tejon Group, ten miles wcst of Griswold's.

VENUS, Linn. V. Veatchi, Gabb.

Venus V'eatchii, Gabb; Vol. 1, p. 162, pl. 23, fig. 142.

Chico Group, Tusean Springs. 
V. Lenticularis, Gabb.

Venus lenticularis, Gabb; Vol. 1, p. 162, pl. 30, fig. 246.

Chico Group, Benicia.

V. TETrAhedra, Gabb.

Venus tetrahedra, Gabb; Vol. 1, p. 163, pl. 30, fig. 247.

Chico Group, Martinez.

V. RQUILATERALIS, Gabb.

Venus aquilateralis, Gabb; Vol. 2, p. 184, pl. 30, fig. 76.

Tejon Group, San Diego.

CHIONE, Mühlf. ? C. Angulata, Gabb.

? Chione angulata, Gabb; Vol. 1, p. 213, pl. 32, fig. 281.

Martinez Group, Martinez.

\section{Varians, Gabb.}

Venus (Mercenaria ?) varians, Gabb ; Vol. 1, p. 161, pl. 23, fig. 140, 141.

Dione varians, Con.; Sm. Chock List, No. 188.

Chico Group, Jacksonville, Siskiyou Mountains, Pence's, Tuscan Springs, Cow Creek, Chico Creek, Folsom, Texas Flat, Benicia, Martinez, Curry's, and Orestimba; Martinez Group, Martinez, and Benicia.

\section{MERETRIX, Lam. M. Uvasana, Con.}

Meretrix Uvasana, Con.; P. R. R. Rep., Vol. 5, p. 320, pl. 2, fig. 3.

Id., Gabb ; Pal. Cal., Vol. 1, p. 163, pl. 30, fig. 248.

Dione Uvasana, Con.; Sm. Check List, No. 187.

Tejon Group, Martinez, Clayton, Cochrane's, Tejon, New Idria, Griswold's, San Diego, and clsewhere.

M. LENS, Gabb.

Meretrix lens, Gabb ; Vol. 1, p. 164, pl. 23, fig. 143.

Chico Group, Chico Creek.

\section{Hornit, Gabb.}

Meretrix Hornii, Gabb ; Vol. 1, p. 164, pl. 23, fig. 144.

$I d .$, Gabb; Vol. 2, p. 185, pl. 30, fig. 78.

Tejon Group, Tejon. 
M. LONGA, Gabb.

Meretrix longa, Gabb; Vol. 1, p. 165, pl. 23, fig. 147.

Chico Group, Texas Flat. (?)

M. ARAta, Gabb.

Meretrix arata, Gabb; Vol. 1, p. 166, pl. 30, fig. 250.

Chico Group, Orcstimba, Siskiyou Mountains, and Cottonwood Creek, Shasta

County.

M. ovalis, Gabb.

Meretrix ovalis, Gabb; Vol. 1, p. 166, pl. 30, fig. 251.

Tejon Group, 'Tejon.

\section{Californica, Con.}

Meretrix Californica, Con.; P. R. R. Rep., Vol. 5, p. 320, pl. 2, fig. 4.

Dione Californiana, Con.; Sm. Check List, No. 174.

Tcjon Group, Tejon.

? M. FraGilis, Gabb.

? Meretrix fragilis, Gabb; Vol. 2, p. 185, pl. 30, tig. 77.

Martinez Group, Martinez.

CARYATIS, Roem. C. Nitida, Gabb.

Meretrix nitida, Gabb; Vol. 1, p. 165, pl. 23, fig. 145, 146.

Caryatis nitida, Gabb ; Vol. 2, p. 186, pl. 30, fig. 79.

Chico Group, Martincz, Orestimba, Chico and Cow Creeks.

Dosinia, Scopoli. D. elevata, Gabb.

Dosinia elevata, Gabb; Vol. 1, p. 167, pl. 30, fig. 252.

D. alta, Con. ; (non Dkr.) P. R. R. Rep., Vol. 5, p. 320, pl. 2, fig. 2.

Dosiniopsis alta, Con.; Sm. Check List, No. 162.

Not Dosinia alta, Con.; Proc. Phil. Acad., 1856, p. 315.

Tejon Group, Tejon.

? D. Tenuis, Meek.

? Dosinia tenuis, Meek; Proc. Phil. Acad., 1861, p. 315.

Chico Group, Vancouver Island. 
D. pertendis, Gabb.

Dosinia pertenuis, Gabb ; Vol. 1, p. 167, pl. 30, fig. 253.

Chico Group, Siskiyou Mountains.

D. Gyrata, Gabb.

Dosinia gyrata, Gabb; Vol. 1, p. 168, pl. 28, fig. 148.

Lucina gyrata, Con.; Sm. Check List, No. 139.

Tejon Group, Martinez, Clayton, Marsh's, Griswold's, Tejon, and San Diego.

D. INfLata, Gabb.

Dosinia inflata, Gabb ; Vol. 1, p. 168, pl. 23, fig. 149.

Chico Group, Chico Creek.

TAPES, Meger]e. T. Conradiana, Gabb.

Tapes Conradiana, Gabb ; Vol. 1, p. 169, pl. 32, fig. 282.

Dione Conradiana, Con.; Sm. Check List, No. 173

Tejon Group, Martinez, Clayton, Tejon, Griswold's ; transition beds between the Tejon and Martinez Group, Lower Lake, Lake County.

\section{? T. QUADRATA, Gabb.}

2 Tapes quadrata, Gabb; Vol. 1, p. 169, pl. 30, fig. 249.

Tejon Group, Martinez, Clayton, Tejon.

\section{? T. cRetacea, Gabb.}

2 Tapes cretacea, Gabb ; Vol. 1, p. 214, pl. 32, fig. 283.

Tejon Group, Corral Hollow.

Thetis, Sby. ? T. elongata, Gabb.

2. Thetis elongata, Gabb; Vol. 2, p. 186, pl. 30, fig. 80 .

Shasta Group, Cottonwood Creek.

TRAPEZIUM, Mühlf. T. CARINATUM, Gabb.

T. carinatum, Gabb; Vol. 1, p. 170, pl. 23, fig. 150.

Chico Group, Texas Flat.

PAL. VOL. II.-32. 
DIODUS, Gabb. D. tenuis, Gabb.

* Cyprinella tenuis, Gabb ; Vol. 1, p. 170, pl. 23, fig. 151.

Tejon Group, Corral Hollow.

\section{CARDIUM, Linn. C. Rémondianum, Gabb.}

Cardium Rémondianum, Gabb; Vol. 1, p. 172, pl. 23, fig. 153.

Chico Group, Benicia, and Wright's Gulch, Shasta County.

\section{Cooperit, Gabb.}

Cardium Cooperii, Gabb; Vol. 1, p. 172, pl. 24, fig. $154 a$.

Tejon Group, Martinez, Tejon, and San Diego.

$$
\text { C. scitulum, Meek. }
$$

C. scitulum, Meek; Trans. Alb. Inst., Vol. 4, p. 40.

Chico Group, Nanaimo, Vancouver Island.

\section{Breweriı, Gabb.}

Cardium Brewerii, Gabb ; Vol. 1, p. 173, pl. 24, fig. 155.

Tejon Group, Martinez, Clayton, Griswold's. and Tejon.

\section{C. (Levicardium) hinteum, Con.}

Cardium linteum, Con.; Pacific R. R. Rep., Vol. 5, p. 320, pl. 2, fig. 1.

Lavicardium linteum, Con.; Sm. Check List, No. 152.

Tejon Group, Tejon.

\section{C. (Lavicardium) annulatum, Gabb.}

Cardium (Lovicardium) annulatum, Gabb; Vol. 1, p. 171, pl. 23, fig. 152. Id., Gabb; Vol. 2, p. 187, pl. 30, fig. 81.

Chico Group, Martinez, Curry's, Orestimba.

\section{C. (Protocardium) Placerensis, Gabb.}

Cardium (Protocardium) Placerensis, Gabb; Vol. 1, p. 173, pl. 24, fig. 156. Chico Group, Texas Flat.

* Cyprinella being preoccupied in ichthyology, I propose to substitute the present vame for the genus. 
C. (Protocardium) translucidum, Gabb.

Cardium (Protocardium) translucidum, Gabb; Vol. 2, p. 187, pl. 30, fig. 82 a. Chico Group, Martinez.

CARDITA, Brug. C. HornIt, Gabb.

C. planicosta, Con. (not Lam.); P. R. R. Rep., Vol. 5, p. 321, pl. 2, fig. 6.

Cardita Hornii, Gabb; Vol. 1, p. 174, pl. 24, fig. 157.

C. Hornii, Gabb; Vol. 2, p. 187, pl. 30, fig. 83 a.

Tejon Group, Martinez, Clayton, Griswold's, New Idria, and Tejon.

C. VENERIFORMIS, Gabb.

Cardita veneriformis, Gabb; Vol. 1, p. 215, pl. 32, fig. 285 a.

Martinez Group, Martinez.

CLIs0colds, Gabb. C. Dubius, Gabb.

Loripes dubia, Gabb ; Vol. 1, p. 177, pl. 24, fig. 170, 171.

Clisocolus dubius, Gabb; Vol. 2, p. 189, pl. 30, fig. 84.

Chico Group, Texas Flat, Chico Creek, and Tuscan Springs.

IUCINA, Brug. I. nasuta, Gabb.

Lucina nasuta, Gabb; Vol. 1, p. 175, pl. 24, fig. 159.

Martinez Group, Martinez.

L. Postice-Radiata, Gabb.

Lucina postradiata, Gabb ; Vol. 1, p. 175, pl. 54, fig. 158.

Chico Group, Texas Flat.

L. SUbcircularis, Gabb.

Lucina subcircularis, Gabb ; Vol. 1, p. 176, pl. 24, fig. 160.

Chico Group, Texas Flat.

L. oumulata, Gabb.

Lucina cumulata, Gabb ; Vol. 1, p. 176, pl. 24, fig. 254.

Tejon Group, Tejon.

? L. cretacea, Gabb.

? Lucina cretacea, Gabb, Vol. 1, p. 177, pl. 30, fig. 255.

Tejon Group, Clayton to Marsh's. 
MYSIA, Leach. ? M. POLITA, Gabb.

? Mysia polita, Gabb; Vol. 1, p. 178, pl. 30, fig. 256.

Tejon Group, Martinez, Clayton, New Idria, and Tejon.

ASTARTE, Sowb. A. Conradiana, Gabb.

Astarte Conradiana, Gabb ; Vol. 1, p. 178, pl. 24, fig. 161. Chico Group, Texas Flat.

\section{A. Mathewsonit, Gabb.}

Astarte Mathewsonii, Gabb ; Vol. 1, p. 179, pl. 30, fig. 258. Chico Group, Martinez.

\section{A. Tuscana, Gabb.}

Astarte Tuscana, Gabb; Vol. 1, p. 179, pl. 30, fig. 257.

Chico Group, Tuscan Springs, Pence's.

ERIPHYLA, Gabb. E. umbonata, Gabb.

Eriphyla umbonata, Gabb; Vol. 1, p. 18, pl. 24, fig. $162 a$.

Chico Group, Cow Creek, and south of Mount Diablo.

CRASSATELla, Lam. C. Grandis, Gabb.

Crassatella alta, Con.; P. R. R. Rep., Vol. 5, p. 321.

C. alta, Con.; Smithsonian Check List, Eocene, No. 104.

Not C. alta, Con.; Tert. Foss., p. 21, fig. 7.

C. grandis, Gabb; Vol. 1, p. 181, pl. 24, fig. 163.

C. grandis, Gabb; Vol. 2, p. 189.

Tejon Group, Clayton, Tejon. Intermediate beds, Lower Lake.

$$
\text { C. compacta, Gabb. }
$$

Crassatella compacta, Gabb; Vol. 2, p. 190, pl. 30, fig. 85 . Martinez Group, Martinez.

\section{Uvasana, Con.}

Crassatella Uvasana, Con.; P. R. R. Rep., Vol. 5, p. 320, pl. 2, fig. 5. Crassatella Uvasana, Gabb; Vol. 1, p. 214, pl. 32, fig. 284.

Tejon Group, Tejon. 
ANTHONYA, Gabb. A. CULTRIFORMis, Gabb.

Anthonya cultriformis, Gabb; Vol. 1, p. 182, pl. 30, fig. 236 a.

Chico Group, Martinez.

UNI0, Retz., Auct. U. Pendutimus, Gabb.

Unio penultimus, Gabb ; Vol. 1, p. 182, pl. 24, fig. 164.

Tejon Group, Coal Mines, Clayton.

\section{U. Hubbardit, Gabb}

Unio Hubbardii, Gabb ; Vol. 2, p. 190, pl. 30, fig. 86.

Chico Group, Coal Mines, Nanaimo, Vancouver Island.

MYTILUS, Linn. M. PaUperculus, Gabb.

Mytilus pauperculus, Gabb; Vol. 1, p. 183, pl. 25, fig. 165.

Martinez Group, Martinez.

M. Ascia, Gabb.

Mytilus ascia, Gabb ; Vol. 1, p. 183, pl. 30, fig. 259.

Tejon Group, Tejon.

M. Humerus, Con.

Mytilus humerus, Con.; P. R. R. Rep., Vol. 5, p 321, pl. 2, fig. 10.

Tejon Group, Tejon.

M. QUAdratus, Gabb.

Mytilus quadratus, Gabb; Vol. 2, p. 191, pl. 31, fig. 87.

Inoceramus Pinchii, Gabb (pars); Vol. 1, pl. 25, fig. 174.

Chico Group, Martinez, and Tuscan Springs

MODIOLA, Lam. M. Siskiyouensis, Gabb.

Modiola Siskiyouensis, Gabb; Vol. 1, p. 184, pl. 30, fig. 260.

Chico Group, Siskiyou Mountains, and Jacksonville.

M. ornata, Gabb.

Modiola ornata, Gabb; Vol. 1, p. 184, pl. 24, fig. 166.

Tejon Group, Martinez, Clayton, Cochrane's, Marsh's, Griswold's, New Idria, and Tejon. 
M. CYLindRica, Gabb.

Modiola cylindrica, Gabb ; Vol. 1, p. 185, pl. 25, fig. 167.

Chico Group, Pence's, Tuscan Springs, Martinez.

\section{MAJOR, Gabb.}

Modiola major, Gabb; Vol. 2, p. 191, pl. 31, fig. 88.

Shasta Group, Lake and Colusa Counties.

\section{LITHOPHAGUS, Mühlf. L. oviformis, Gabb.}

Lithophagus oviformis, Gabb; Vol. 1, p. 185, pl. 25, fig. 168.

Chico Group, Cow Creek.

SEPTIFER, Recluz. S. рIснотомus, Gabb.

Septifer dichotomus, Gabb; Vol. 1, p. 186, pl. 30, fig. 261.

Tejon Group, Tejon.

STALAGMIUM, Con. S. Concentricum, Gabb, sp.

Crenella concentrica, Gabb; Vol. 1, p. 186, pl. 24, fig. 169.

Stalagmium concentricum, Con.; Sm. Check List, No. 96.

Tejon Group, Martinez.

AVicula, Lam. A. Pellucida, Gabb.

Avrcula pellucida, Gabb; Vol. 1, p. 186, pl. 25, fig. 172

Tejon Group, Martinez, Griswold's, Tejon; transition beds, Lower Lake; Martinez Group, Martinez; Chico Group, Siskiyou Mountains, San Luis, Gonzaga.

MELEAGRINA, Lam. M. ANTiqua, Gabb.

Meleagrina antiqua, Gabb; Vol 2, p. 192, pl. 31, fig. 89.

Chico Group, Nanaimo, Vancouver Island.

INOCERAMUS, Sowb. I. ELLIOTII, Gabb.

Inoceramus Elliotii, Gabb; Vol. 2, p. 193, pl. 31, fig. $90 a$.

Chico (?) Group, Aleatraz Island. 
I. Whitneyi, Gabb.

Inoceramus Whitneyi, Gabb; Vol. 2, p. 193, pl. 31, fig. 91.

Chico Group, Folsom.

I. subundatus, Meek.

Inoceramus subundatus, Meek; Proc. Phil. Acad., 1861, p. 315.

Chico Group, Vancouver Island.

AUCELla, Keys. A. Piochir, Gabb.

Inoceramus Piochii, Gabb; Vol. 1, p. 187, pl. 25, fig. 173 (exclus. fig. 174).

Aucella Piochii, Gabb; Vol. 2, p. 194, pl. 31, fig. 92, a-c.

Shasta Group, Mitchcll Cañon, north side of Mount Diablo; Knoxville, Puta

Creek, and clsewhcre in Lake County; near thc Simmons' Springs, and elsewhere, Colusa County, and at numerous other spots along the eastern face of the northern Coast Range; also southwest of San Jose, below the Almaden mines; and from Washington Territory.

PINNA, Linn. P. BreweriI, Gabb.

Pinna Brewerii, Gabb ; Vol. 1, p. 188, pl. 25, fig. 175 a.

Id., Gabb; Vol. 2, p. 195, pl. 32, fig. 93.

Chico Group, Curry's, south of Mount Diablo, Martincz, Cottonwood Creek, Shasta County, and Cottonwood Creek, Siskiyou County.

Trigonia, Brug. T. Tryoniana, Gabb.

Trigonia Tryoniana, Gabb; Vol. 1, p. 188, pl. 25, fig. 176.

Cbico Group, Tuscan Springs.

\section{T. Evansana, Meek.}

Trigonia Evansana, Meck; Trans. Albany Inst., Vol. 4, p. 42.

T. Evansii, Gabb; Vol. 1, p. 189, pl. 25, fig. 177.

One of the most characteristic fossils on the West Coast, peculiar to the Chico

Group, and found in California, at Pacheco's Pass, south of Mount Diablo, Orcstimba, Martinez, Benicia, Tuscan Springs, Chico Creek, Pencc's, Cottonwood Creek, Shasta County, Cottonwood Creek, Siskiyou County, Texas Flat, and Rag Cañon; in Oregon, at Jacksonville, on the Siskiyou Mountains, and on the Crooked River of the Des Chutes; and finally, on Vancouver Island, at Nanaimo, whence it was described by Mr. Meek. 


\section{T. Gibboniana, Lea.}

Trigonia Gibboniana, Lea ; Trans. Am. Phil. Soe., 1840, p. 255, pl. 9, fig. 7.

T. Hondaana, Lea; loc. cit., p. 256, pl. 9, fig. 9.

T. Hondaana, d'Orb. ; Prod. Pal. Strat., Vol. 2, p. 106, No. 771.

T. Gibboniana, Gabb; Vol. 1, p. 190, pl. 17, fig. 178; pl. 31, fig. 262.

Chieo Group, Martinez, Jaeksonville, and Crooked Creek of the Des Chutes.

\section{T. AQUicostata, Gabb.}

Trigonia axquicostata, Gabb; Vol. 2, p. 196.

Indet., Vol. 1, p. 209, pl. 26, fig. 198.

Chieo Group, Orestimba, south of Mount Diablo, Martinez, Cottonwood Creek, Shasta County, and Jaeksonville, Oregon.

MEEKIA, Gabb. M. SELLA, Gabb.

Meekia sella, Gabb; Vol. 1, p. 191, pl. 25, fig. 179.

Martinez Group, Martinez; Chieo Group, Tusean Springs, and Siskiyou Mountains.

M. RAdiata, Gabb.

Meekia radiata, Gabb; Vol. 1, p. 192, pl. 25, fig. 179 a.

Chieo Group, Paeheeo's Pass, Orestimba, Tusean Springs, Siskiyou Mountains, and Jaeksonville, Oregon.

M. NAvis, Gabb.

Meekia navis, Gabb; Vol. 1, p. 192, pl. 25, fig. 180.

Chieo Group, Martinez, Chieo Creek, and Penee's.

ARCA, Lam. A. Breweriana, Gabb.

Arca Breweriana, Gabb; Vol. 1, p. 193, pl. 25, fig. 181.

Chieo Group, Cottonwood Creek, Tusean Springs.

A. Gravida, Gabb.

Arca gravida, Gabb; Vol. 1, p. 194, pl. 30, fig. 264.

Chico Group, Paeheeo's Pass, and Rag Cañon, Napa County.

\section{A. Decurtata, Gabb.}

Arca decurtata, Gabb; Vol. 1, p. 195, pl. 31, fig. 265 a.

Chieo Group, Rag Cañon. 


\section{A. Hornit, Gabb.}

Arca Hornii, Gabb; Vol. 1, p. 194, pl. 30, fig. 263.

Tejon Group, Tejon.

\section{A. Vancouverensis, Meek.}

Arca Vancouverensis, Meek; Trans. Alb. Inst., Vol. 4, p. 40.

Chico Group, Nanaimo, Vancouver Island.

Barbatia, Gray. B. Morser, Gabb.

Barbatic Morsei, Gabb; Vol. 1, p. 216, pl. 32, fig. 286.

Tejon Group, San Dicgo.

CUCUlläa, Lam. C. Mathewsonit, Gabb.

Cucullcea Mathewsonie,-Gabb; Vol. 1, p. 195, pl. 31, fig. 266.

Intermediate beds between the Tejon and Martinez Groups, Clayton, and Lower Lake; Martinez Group, Martinez.

\section{C. truncata, Gabb.}

Cuculloen truncata, Gabb; Vol. 1, p. 196, pl. 25, tig. 182.

Chico Group, Pacheco's Pass, Orestimba, south of MIount Diablo, Martinez, Benicia, Texas Flat, Tuscan Springs, and Jacksonville, Oregon.

$$
\text { C. equilateralis, Meek. }
$$

Arca (Cucullcea) equilateralis, Mcek ; Trans. Alb. Inst., Vol. 4, p. 39.

Chico Group, Nanaimo, Vancouver Island.

aXINeA, Poli. A. Veatchi, Gabb.

Axincea Veatehii, Gabb; Vol. 1, p. 197, pl. 25, fig. 183 a.

Beds intermediate betwcen the Tejon and Martinez Groups, Lower Lake;

Martinez Group, Martinez; Chico Group, Tuscan Springs, Texas Flat, Pence's, Cow Creek, Orestimba, and San Diego.

A. sagittata, Gabb.

Axincea (Limopsis?) sagittata, Gabb; Vol. 1, p. 197, pl. 31, fig. 267 a.

Axincea sagittata, Gabb; Vol. 2, p. 196.

Tejon Group, Tejon, Griswold's, and Martinez.

PAL. VOL. II. -33 


\section{A. Cor, Gabb.}

Axinœa cor, Gabb; Vol. 1, p. 198, pl. 31, fig. 268 a.

Tejon Group, Martinez.

NUCUIA, Lam. N. Traskana, Meek.

Nucula Traskana, Meek; Trans. Alb. Inst., Vol. 4, p. 39.

Chico Group, Nanaimo, Vancouver Island.

\section{N. solitaria Gabb.}

Nucula solitaria, Gabb; Vol. 2, p. 197, pl. 32, fig. 94.

Chico Group, Texas Flat.

$$
\text { N. (Acila) truncata, Gabb. }
$$

Nucula truncata, Gabb; Vol. 1, p. 198, pl. 26, fig. 184, a, b.

N. (Acila) truncata, Gabb; Vol. 2, p. 197.

Chico Group, Pence's, Tuscan Springs, Chico Crcek, Texas Flat, Pacheco's

Pass; Martinez Group, Martinez; Tejon Group, Martinez, and Tejon.

\section{IEDA, Schum. T. Gabbir, Con., sp.}

Leda protexta ?, Gabb; Vol. 1, p. 199, pl. 26, fig. 185.

Not L. protextu, Gabb; Jour. Phil. Acad., '2 Ser., Vol. 4, p. 303, pl. 48, fig. 23. Nuculana Gabbii, Con.; Smithsonian Check List, No. 44.

Martinez Group, Martinez; 'I'jon Group, Martincz, Clayton, Griswold's, Tejon, and San Emidio.

$$
\text { I. Translucida, Gabb. }
$$

Leda translucida, Gabb ; Vol. 1, p. 199, pl. 30, fig. 269.

Chico Group, Cov Crcek.

\section{LIMOPSIS, Sassi. I. transversa, Gabb.}

Limopsis transversa, Gabb; Vol. 1, p. 200, pl. 26, fig. 186.

Chico Group, Texas Flat.

\section{peCten, Brug. P. Traskit, Gabb.}

Pecten Traskii, Gabb; Vol. 1, p. 200, pl. 26, fig. 187 a. Il., Gabb ; Vol. 2, p. 198, pl. 32, fig. 95.

Chico Group, Texas Flat, and Nanaimo, Vancouver Island. 


\section{P. operculiformis, Gabb.}

Pecten operculiformis, Gabb; Vol. 1, p. 201, pl. 26, fig. 188.

Chico Group, Cottonwood and Huling Creeks, and south of Mount Diablo.

\section{P. Californicus, Gabb.}

Pecten Californicus, Gabb; Vol. 1, p. 201, pl. 31, fig. 270.

Chico or Shasta Group, Cottonwood Creek, Shasta County.

\section{P. Martinezensis, Gabb.}

Pecten Martinezensis, Gabb ; Vol. 2, p. 198, pl. 33, fig. 96. Martinez Group, Martinez.

$$
\text { P. complexicosta, Gabb. }
$$

Pecten complexicosta, Gabb; Vol. 2, p. 199, pl. 33, fig. $97 a$. Shasta Group, Morgan Valley, Lake County.

\section{P. interradiatus, Gabb.}

Pecten interradiatus, Gabb; Vol. 2, p. 199, pl. 33, fig. 98 a. Tejon Group, New Idria.

NEITHEA, Dronet. N. Grandicosta, Gabb.

Neithea grandirosta, Gabb ; Vol. 2, p. 200, pl. 33, fig. 99 a. Shasta Group, Cottonwood Creek.

\section{LIMA, Brug. L. Microtis, Gabb.}

Lima microtis, Gabb; Vol. 1, p. 202, pl. 26, fig. 189.

Chico Group, Cottonwood Creek (and Texas Flat?).

L. APPRESSA, Gabb.

Lima appressa, Gabb; Vol. 1, p. 203, pl. 31, fig. 271.

Chico Group, Pacheco's Pass.

\section{Shastaensis, Gabb.}

Lima Shastaensis, Gabb; Vol. 2, p. 201, pl. 33, fig. 100. Shasta Group, Cottonwood Creek. 
L. mulitiradiata, Gabb.

Lima multiradiata, Gabb ; Vol. 2, p. 201, pl. 33, fig. 101.

Transition beds between Tejon and Martinez Group, Lower Lake, Lake County.

pitcatula, Lam. P. variata, Gabb.

Plicatula variata, Gabb; Vol. 1, p. 203, pl. 26, fig. 190.

Shasta Group, Battle Creck, Shasta County.

ANOMIA, Lino. A. Lineata, Gabb.

Anomia lineata, Gabb; Vol. 1, p. 203, pl. 26, fig. 193.

Chico Group, Texas Flat, Pence's, Chico Crcek.

A. Vancouverensis, Gabb.

Anomia Vancouverensis, Gabb; Vol. 2, p. 202, pl. 33, fig. 102.

Chico Group, Nanaimo, Vancouver Island.

PLacUnanomia, Brod. P. inornata, Gabb.

Placunanomia inornata, Gabb; Vol. 1, p. 217, pl. 32, fig. 288 a.

Tejon Group, Corral Hollow, and San Diego.

ostrea, Linn. U. Brewerit, Gabb.

Ostrea Brewerii, Gabb; Vol. 1, p. 204, pl. 26, fig. 191.

Chico Group, Cow Creek.

O. MALleiformis, Gabb.

Ostrea malleiformis, Gabb ; Vol. 1, p. 204, pl. 31, fig. 272.

Chico Group, Cotton wood Creek, Siskiyou County, and Jacksonville, Oregon.

\section{O. IDRIAensis, Gabb.}

Ostrea Idriaensis, Gabb; Vol. 2, p. 203, pl. 33, fig. 103, b, c; pl. 34, fig. $103 a$. Tejon Group, New Idria.

$$
\text { O. appressa, Gabb. }
$$

Ostrea appressa, Gabb; Vol. 2, p. 203, pl. 34, fig. 104 a.

Tejon Group, Mendocino County. 
GRYPH EA, Lam. G. VESICULARIS, Lam., sp.

Ostrea vesicularis, Lam.; An. de Mus., Vol. 8, p. 160, pl. 22, fig. 3.

Gryphcea vesicularis, Bronn; Leth. Geog., pl. 32, fig. 1.

Chico (?) Group, San Diego.

EXogYRA, Say. E. Parasitica, Gabb.

Exogyra parasitica, Gabb; Vol. 1, p. 205, pl. 26, fig. 192, $\alpha, b$; pl. 31, fig. $273 \alpha$.

Chico Group, Texas Flat, Folsom, Cottonwood Creek.

\section{BRACHIOPODA.}

TEREBRATELLA, d'Orb. 'T. oBesa, Gabb.

Terebratella obesa, Gabb ; Vol. 1, p. 205, pl. 26, fig. 194.

Chico Group, Texas Flat.

RHYNCHONELLA, Fisch. R. WIIITNEYI, Gabb.

Terebratella Whitneyi, Gabb; Vol. 2, p. 35, pl. 12, fig. $62 \alpha$.

Rhynchonella Whitneyi, Gabb; Vol. 2, p. 204, pl. 34, fig. 135, a, b.

Shasta Group, Lake and Colusa Counties.

\section{ZOOPHYTA.}

FLABELLUM, Lesson. F. Rémondianum, Gabb.

Flabellum Rémondianum, Gabb; Vol. 1, p. 207, pl. 26, fig. 199.

Tejon Group, Clayton.

SMILOTROCHUS, E. \& H. ? S. curtus, Gabb.

Smilotrochus curtus, Gabb; Vol. 2, p. 205, pl. 34, fig. 106 a.

Martinez or Chico Group, Martinez.

Trochosmilia, E. \& H. T. (Acrosmilia) striata, Gabb.

T. (A.) striata, Gabb; Vol. 1, p. 207, pl. 26, fig. 195.

Tejon Group, Clạyton. 
T. (Ellipsosmilia) granulifera, Gabb.

T. (E.) granulifera, Gabb; Vol. 1, p. 208, pl. 26, fig. $196 a$. Chico Group, Chico Creek.

ASTRocenia, E. \& H. ? A. PETrosa, Gabb.

2 Astroconia petrosa, Gabb ; Vol. 1, p. 208, pl. 31, fig. 274 a. Martinez Group, Martinez. 


\title{
PALEONTOLOGY OF CALIFORNIA.
}

\author{
V 0 L. II.
}

SECTION III.

DESCRIPTION OF CRETACEOUS FOSSILS FROM MEXICO. 



\title{
NOTES ON SOME
}

\section{MEXICAN CRETACEOUS FOSSILS,}

\author{
WITH DESCRIPTIONS OF NEW SPECIES,
}

THE following fossils were collected by my lamented friend, RÉmond, several years ago, in the Sierra de las Conchas, near Arivechi, Sonora. When they were first received, I published a short notice of them, in the Proceedings of the California Academy of Natural Sciences, accompanied by some extracts from M. RÉmond's letter, and mentioned the close relationship that obviously exists between this deposit and the Cretaceous of Texas; at the same time giving a list of the species identified, in hastily glancing over the collection. Although sufficiently accurate for the purpose, the list contained one or two errors in identification of species, due to the imperfect character of some of the specimens, in the first instalment received. In the present paper I have endeavored to correct the errors, and believe that I have correctly determined all the previously known species.

The occurrence of the Texan Cretaceous fauna on the western face of the Sierra Madre, is a matter of great interest, since it proves conclusively that during that era there must have been a water communication between the great Cretaceous sea, that covered so much of what is now the central portions of our continent, on the one side, and the Pacific on the other. It is the more remarkable when taken in connection with the fact, that of the more than three hundred species now known in the Californian Cretaceous, barely one per cent. is found in common on the two sides of the continent. From the occurrence in California of Gryphoea vesicularis and Turritella seriatim-granulata, determined 
with eertainty, and of Nautilus Texanus and Volutilithes Navarroensis, yet open to a doubt, it seems that there was not a continuous land barrier between the two basins. Three out of the four speeies enumerated, are from the Texan fauna, and are not known so far north as Nebraska, though beds of equivalent age are found in that Territory. It is very probable that future explorations in the yet unknown region between the Saskatelawan and the Pacific, north of our boundaries, will develop a more or less continuous series of Cretaeeous deposits, showing a similar link on the north. The presence of Ammonites complexus on Vancouver Island, and in California, and the known existenee of Cretaceous beds in Eastern Oregon, and northwest of the great lakes, render this hypothesis not improbable.

I need say but little about the age of this deposit. Dr. Roemer, in his admirable work, "Kreidebildungen von Texas," has diseussed the subjeet in so able a manner as to leave me nothing to add. He eonsiders it to be on, or near, the horizon of the Lower Chalk; an opinion in whieh I fully coneur.

So far as I am aware, this is only the second locality of Cretaeeous roeks reported in the whole area of Mexieo. In 1839, GaLEotti published a paper in the Bulletin of the Brussels Aeademy, on some Jurassic fossils from Tehuaean, in the state of Puebla, some of whieh, at least, seem to belong to the Cretaeeous, rather than to the Jurassie formation.

\section{AMMONITES, Brug.}

\section{A. Pedernalis, Von Bueh.}

Pl. 35, Fig. 1, 1 a.

(A. Pedernalıs, Von Buch; Ueber Cerat., p. 31, pl. 6, fig. 8-10.)

(Id., Roem; Kreid. Tex., p. 34, pl. 1, fig. 3, a-c.)

(Id., Con. ; Emory's Report, pl. 15, fig. 1.)

(Id., Gabb ; Synop. Cret., Philos. Proe.; 1861, p. 14.)

(A. pleurisepta, Con.; Emory's Report, p. 159, Pedernalis on plate.)

A peculiar variety of this species, differing both from the figures in Kreid. 
Texas, and that by Conrad in the Mexican Boundary Report, in having the dorsum distinetly channelled or flattened, and bordered by two angles, and in baving the sides variously undulate or even subcostate. I have seen the flattened dorsum in Texan specimens; and the undulations of the sides, on the specimens beforc me, show by their variability that this is a character of no spccific value. The septum is identical with the published figures.

\section{FUSUS, Lam.}

\section{F. Mexioanus, n. s.}

Pl. 35, Fig. 2.

SHeLl small, elongate fusiform; spire high, whorls five or six, angulated, top sloping; suture undulated. Surface ornamented by about ten large radiating ribs, crossed by numerous prominent revolving lines with broad interspaces; the longitudinal ribs commence at the suture and, on the body whorl, disappear about the middle. Aperture broad and biangular posteriorly, narrowed in advance; outer lip simple, inner lip incrusted; canal produced.

Length (both ends broken), .75 inch.

A peculiar specics, having no near allies either in California or Texas.

\section{LUNATIA, Lam. \\ L. Pedernalis, Roem., sp.}

Pl. 35, Fig. 3.

(Natica Pedernalis, Roem.; Kreid. Tex., p. 43, pl. 4, fig. 1.)

Dr. Poemer scems to have been acquainted with this species only through internal casts, one of which he figures as above. I am fortunate in having lad the opportunity of examining some half a dozen specimens retaining all the shell. The species is very thin, and nearly all have suffered more or less distortion. The specific characters are as follows.

SHELL large, ovate, thin; spire moderately produced; whorls five and a half, rounded; suture distinct. Aperture elongate ovate, acute behind, rounded in advance; outer lip simple, inner 
lip broadly incrusted above, thiekened in front of, and below the umbilicus. Umbilicus subperforate, a thin striate incrustation running up into it, and extending below, parallel with the anterior portion of the labium. Surfaee marked by faint lines of growth.

This is by far the largest Naticoid in the American Cretaceous, and, apart from its size, can be distinguisled from all the other species by its elongate form and the striate umbilicus.

\section{EUSPIRA, Agas.}

E. tabulata, n. s.

Pl. 35, Fig. 4.

SheLl thick, robust, pyriform; spire elevated turrited; whorls five; upper surface directly or obliquely truneated; suture faint. Bodly whorl flat above, concave immcdiately below the angle, and broadly expanded towarls the base. Surfaee plain. Aperture biangular behind, rapidly widening, and round in front; outer lip simple, but rather thick; inner lip inerusted narrowly above, thickened over, and in front of the umbilieus. Umbilieus imperforate, a small angular rilge revolving from the umbilicus, nearly parallel with the margin of the inner lip.

Figure, natural size.

A rare shell, and entirely unlike any other in the formation. It seems to be most closely allied to the Oolitic genus Euspira, and al together looks much more like a Jurassic than like a Cretaeeous species.

CHEMNITZIA, d'Orb.

C. ZeBrA, n. s.

Pl. 55, Fig. 5.

SHELL elongate, slender-conieal; spire high, whorls numerous, flattened, or very slightly convex on the sides; suture linear, impressed; body whorl flattened above, rounded below. Surface 
plain, marked only by obsolete lines of growth, and ornamented by more or less sinuous, broken, irregular bands of color, longitudinally disposed, and sometimes entire, sometimes zigzag, branching, anastomosing and running out. Aperture subelliptical; outer lip sinuous; inner lip heavily incrusted.

Figure, natural size.

Easily recognized by its elongate form and nearly flat sides, and by the unusual circumstancc of the retention of its color. Every spccimen I have scen shows the colored pattern when the surface is wet, and most of them retain it so strongly as to show it even when dry.

Considerable confusion has arisen from a want of a clear understanding of the relations of d'Orbigny's genus Chemnitzia to Turbonilla of Risso. I believe Mr. Conrad was the first author to call attention to the fact, that good points of difference exist between the two. Chemnitzia is essentially a genus of comparativcly large shells, all fossil, and which seem to be confined to Secondary rocks. They have thick shells, and are usually more or less angulated, with heavily incrusted inner lips. Turbonilla seems to have originated in the later Tertiaries, and attains itsmaximum development in the present seas. It contains only small shells, many of them being almost microscopic; they are usually thin, and the inner lip is little or not at all incrusted.

\author{
C. Texana, Roem., sp. \\ (Scalaria Texana, Roem.; Kreid. Tex., p. 39, pl. 4, fig. 11.)
}

A smaller shell than the preceding; characterized by rounded whorls, strong longitudinal ribs, and smaller revolving ribs. It secms to bc a rather rare shell.

\title{
TYLOSTOMA, Sharpe. 1849.
}

Varigera, d'Orb. 1850.

T. MUTABILIS, n. s.

Pl. 35, Fig. 6, $a, b, c$.

Snelu very variable in shape, according to age. In the very young, subglobose or subovate; becoming more elongate in the adult, and compressed in the very old; apex elevated in average specimens; whorls seven to seven and a half, rounded on the sides, and usually more or less converging; suture deeply im- 
pressed; transverse seetion subcireular to subelliptieal, the two variees being nearly linear or obsolete in the young; and rough, prominent, and sometimes imbricating in the adult. Aperture elongate, acute behind, narrowly rounded in advanee; outer lip simple or slightly thiekened. In one very small specimen, the inner surfaee of the outer lip shows a minute erenulation, that I have been unable to deteet in any other speeimens of a large series. Inner lip slightly inerusted. Surface marked by lines of growth, and by the variable varices.

Figures 6 and $6 c$, natural size. Figure 6 , from an average speeimen, $6 c$, from an old shell, a top view to show the extreme form of the variees; $6 a$ and $6 b$, front and top views, magnified, from a young shell, showing the in ternal erenulation and the symmetrieal form.

One of the most common fossils at Ariveehi.

\section{ANCIIURA, Conrad.}

A. MONilifera, n. s.

Pl. 35, Fig. 7.

SHeLl small, elongate, slender, fusiform; spire elevated; whorls numerous (six or seven?), rounded on the sides; body whorl regularly rounded, never angulated except in some instances near the origin of the large lip, where a faint cariua starts and runs out in the middle of the lip. Surface ornamented by numerous, slightly flexuous, rounded ribs, with equal, eoncave interspaces, ribs and spaces alike crossed by about a dozen sharply elevated revolving threads. Outer lip unknown; the posterior angle of the mouth reaches almost to the penultimate suture, and both posteriorly and on the inner lip the aperture is borderer by a rather broad and somewhat flattened plate. Anterior canal unknown.

Although this shell is quite eommon; among perhaps fifty speeimens, there was not a single one showing more of the outer lip, or of the eanal, than is illustrated in the figure. 


\section{CERITHIUM, Brug.}

\section{Mexicanum, n. s.}

P1. 35, Fig. 8.

SHell elongate, slender; whorls numerous, rounded on the sides; suture linear, impressed. Surface marked by numerous, slightly curved longitudinal ribs, about twenty-tive to a volution; these are crossed by tive or six revolving ribs, which produce a small node at each crossing of the longitudinal ribs; between each pair are two or three minute revolving lines. On each volution there is one longitudinal rib, larger than the others, which seems to be a regular varix; these are irregularly placed, but on six whorls there are but six of these varices. They are not constant in size, and on the upper whorls, where the longitudinal ribs are less numerous, and proportionally larger, they assimilate them more nearly in appearance. Aperture broad, inner margin somewhat thickened and flattened.

Length of fragment, less anterior portion of aperture and several apical whorls, 1.1 inch.

Rare. But two or three specimens were found.

\section{TURRITELLA, Lam.}

\section{T. Seriatim-Granulata, Roem.}

Under this head, among the Californian fossils, I have already given the synonymy of this species: see the enumeration (ante). The shell is very common, and is extremely variable in the details of its ornament, both in the number and in the arrangement of its ribs. Its apical angle is also somewhat variable. 
ANGARIA, Bolt.

Delphinula, Lam.

A. cingulata, n. s.

Pl. 35, Fig. 9.

SHell moderate in size, compact, whorls all in close contact; spire moderately elevated; upper whorls rounded; body whorl angulated, flattened above and on the sides, internally rounded; umbilicus broad, bordered by a sharp angle. Surface ornamented on the sides by large, oblique, longitudinal ribs or undulations, and on the top, sides, and bottom by numerous small revolving ribs; two, larger than the others, on the umbilical margin. Aperture large, subangulated, rounded in advance; inner lip sinuous.

Figure, natural size.

Rare. A single specimen in the collection of Mr. F. L. A. Pioche, of San Francisco. Well characterized by the whorls being all in close contact, by its subangular form, and by the obliquely undulated surface.

\section{CINULIA, Gray. Avellana, d' Orb. \\ C. Rectilabrum, n. s. Pl. 35, Fig. 10, $10 a$.}

Shell small, compact, solid, subglobose ; spire moderately elevated; whorls four, rounded; suture impressed. Surface marked by numerous large revolving ribs, in the interspaces of which can be sometimes detected a few obsolete punctations. Aperture elongate, narrow behind, rounded in advance; outer lip broad, thick, nearly straight; inner lip heavily incrusted, and bearing in advance two large, prominent folds; anterior margin of the mouth slightly emarginate. 
Length, .4 inch; greatest width, . 32 incle; lesser, . 24 inch.

This species is allied to $C$. Mathewsonii of the California rocks, but differs in its less compressed form, the presence of but two folds in the mouth, placed in a different manner, and in the details of the sculpturc.

\section{PHOLADOMYA, Sowb.}

\section{P. Sonorensis, n. s.}

Pl. 36, Fig. 12.

Shell elongate, suboval, very oblique, gibbous; beaks onefourth of the length from the anterior end, which slopes nearly straight from the beaks to the middle, and is narrowly rounded or subtruncate below; cardinal margin concave; base irregularly convex, most prominent at, or a little posterior to the middle; posterior end regularly rounded. Surface marked by uumerous, prominent, rounded, irregularly disposed, concentric ribs, crossed by four or five faint radiations, dividing the surface into as many obsolete plancs. In some cases, the spaces between the radiating lines are faintly concave.

This shell is quite rare. I have seen but two or threc specimens. It is not unlike P. Oregonensis, supra (Pl. 29, fig. 65), but the bcaks arc less anterior, the cardinal margin is more convex, the posterior end is more regularly rounded, and the concentric ribs are strong and prominent, instead of being nearly obsolete, as in that species.

\section{TAPES, Megerle.}

T. Hilgardi, Shum.

Pl. 36, Fig. 13.

(Tapes Hilgardi, Shumard; Trans. St. Louis Acad., 1860, p. 601.)

The prcsent shell agrees with Dr. Shumard's description, and with a sketch which Mr. A. R. Roessler, late assistant in the Texas Survey, was kind enough to lend me, and which he made from the original type. Following Dr. Shumard, I refer the species to Tapes, though the hinge is not yet known, and I am confident that a thorough revision will bc required among the numcrous species referred to the various genera of Veneridce, where generic distinctions are based so exclusively on the binge, and where external form is of so little value. The recent PAL. VOL. II. -35 
researehes of Dr. Roemer and Mr. Conrad, have demonstrated the existence of several well-eharaeterized and peeuliar genera in the Cretaeeous and Tertiary, among forms, until recently, referred to modern genera; and were our material more perfect, I am eonvinced that still further separations would be required. lalæontologieal eonchology is shamefully in the rcar, in the matter of generie division, as eompared with that portion of the seienee which relates to living forms; and although palæontologists have a good exeuse for thcir eonservatism, in the very frequent total or partial absence of claraeters, yet when the material under eonsideration does retain all of its distinctive eharaeters, they are too apt to allow their fear of radiealism, on the side of exeessive subdivision, to drive them into the no less blameworthy fault of "old fogyism" on the other extreme.

It is in view of this failing of my eo-laborers in the ficld of fossil conehology, that I have proposed so many new genera and sub-genera in the present work. I hardly lope that, for the present, all of them will be adopted, though they are certainly as distinct as the average of the modern genera, universally aeknowledged by the students of the existing malaeological fauna.

\section{CARDIUM, Linn.}

\section{GRANOCARDIUM, New S. Gen.}

I propose the present subdivision of Cardium to reccive a wellmarked, though small group of shells in the genus, characterized by a peculiar style of surface ornament. They seem to be peculiar to the upper portions of the Cretaccous formation, the oldest species with which I am aequainted being found in the upper Green Sand. The subgenus may be described as follows: Shell nearly equilateral, usually longer than wide; valves elosed all round; surface ornamented by two series of radiating ribs, large ribs bearing spines, tubercles, or grains, and smaller ribs occupying the interspaccs between the larger, and also granulate.

This is one of the most highly ornamented groups in the genus, and is represented in Europe by C. productum, Sby., C. Moutonianum, d'Orb, and C. Carolinum, d'Orb, and in Ameriea by C. Tippanum, Conrad, and the present speeies. Tippanum is from the equivalent of the uppcr clalk, sabulosum is on the horizon of the lower elialk, and the three European species quoted, are from the upper Green Sand, (Cenomanien, d'Orb.).

The ornaments of the present species are more minute than on any of the others in the subgenus, with whieh I am aequainted. 
C. (G.) sabulosum, n. s.

Pl. 36, Fig. 14

Shell convex, nearly equilateral, rounded, subquadrate; beaks central, slightly pointed in advance; umbones very prominent; cardinal margins equally and very slightly sloping; anterior end and base regularly convex; posterior end rounded, subtruncate. Surface marked by from twenty to thirty radiating ribs bearing small, elongate tubercles; between these ribs are broad, flat, interspaces, each one with two or three fine, linear ribs, minutely grauulate.

Figure, natural size; transverse diameter, 1.15 inch.

\section{C. (Protocardium) granuliferum, n. s.}

Pl. 36, Fig. 15.

Shell large, thin, subquadrate, subcompressed. Beaks central, strongly pointed in advance, umbones moderate; cardinal margins sloping; base broadly and regularly rounded; anterior end rounded, sometimes rounded subtruncate; posterior end obliquely truncate. Surface marked by from fifteen to eighteen radiating ribs, which occupy the posterior face to beyond the posterior basal angle; some of these ribs, those nearest the outer margin of the shell, carry small granulations, irregularly disposed along their leugth. The remainder of the surface is covered by irregular lines of growth.

Figure, natural size of an avcrage sjccimen. One finc example before me measures length and width each, 3 inches, diametcr of two valves, 1.8 inch. $\Lambda$ small specirncn, less than an inch in length, has the beaks much less prominent than in the figure, the cardinal margins much more sloping, and the wholc outline below forming a regular curve. On comparing the specimens with the figures of $C$. (P.) multistriatum, Shum., in Emory's Report, pl. 6, fig. $4 a-c$, the present form scems to be more truncated posteriorly, and I can detect no indications of the granules on the radiating ribs either in the figures or in the descriptions of Conrad or Shumard. By comparing with some very imperfect specimens of Shumard's 
species, I find a great difference in the convexity; that form having a far greater transverse diameter. I have never been so fortunate as to see a good example of the Texan shell; and have been obliged to rely on the descriptions and figures in Marey's and Emory's Reports, almost entirely. It is not impossible that the two species may prove identical, though I hardly think it possible that, had it existed, so marked a character as the granules on the ribs would have been overlooked by two such students as Conrad and Shumard.

\section{CARDITA, Brug.}

\section{?C. Alticosta, Il. s.}

Pl. 36, Fig. 16.

Sinell oblique, subtrigonal, gibbous; beaks prominent, subterminal; anterior end deeply excavated under the beaks, broadly and prominently rounded below; cardinal margin rapidly sloping, nearly straight; posterior end narrow, rounded; base regularly convex. Surface marked by about twenty-five high, narrow ribs with concave interspaces. In some cases these ribs are smooth, in others they are crossed by lines of growth, developed into imbricating plates. Lunule small, broad, cordate.

Length, .8 to .9 inch.

Common. Although I have becn unable to expose the hinge of this shell, I have very little doubt of the correctness of the gencrie reference. Externally it possesses every character of Cardita. The shell is invariably crystalline, and the matrix is a tough amorphous limestone, which never yiclds under the chisel until the shell is completely shattered; I liavc, in consequenec, been obliged to trust to external resemblanees, rather than to the more certain evidence furnished by the internal characters.

\section{PINNA, Linn.}

\section{P. SP. INDET.}

Fragments of a long slender Pinna are not rare in the collection. They indicate a species not unlike $P$. Brewerii nob., but are in too fragmentary a condition to warrant description. 


\title{
TRIGONIA, Brug.
}

\author{
T. Mooreana, Gabb.
}

(T. crenulata, Roem. (not Lam.); Krejd. Tex., p. 51, pl. 7, fig. 6.)

(T. Mooreana, Gabb; Synopsis Cret. Moll., p. 176.)

Shelc elongate subtrigonal, beaks small, terminal, incurved; anterior end slightly convex, rapidly sloping backwards; base forming a regular curve, continuous with the anterior margin; posterior cardinal margin broadly and slightly concave; posterior end unknown. Surface ornamented by about twenty-five moderately large and slightly sinuous ribs, very slightly crenulated, with broad concave interspaces. These ribs become obsolete on the border of the corselet, or cross it transversely as finc lines, continuing across the central arca as sharply defined, linear ribs, in a transverse direction, slightly curved forwards at their extremities. Corselet broad, nearly flat, with a narrow border, marked by a deep longitudinal groove.

Length of a broken specimen, less a portion of the posterior end, 2.4 inches, probable total length, 2.8 inches; width, 1.5 inch, height of single valve, .9 inch.

Not rare at Arivechi.

The present species belongs to a large group in the genus, all of which are more or less closely allied. It can be distinguished from $T$. crenulata, its nearest ally, and to which Dr. Rocmer refcrred it, by its more slender form, less prominent base, less strongly crenulated ribs, and by thc character of the corselet. In Lamarck's species, that area is comparatively smaller, and is bordercd by a wide margin, over which the ribs cross very obliquely backwards from the bcak, afterwards crossing the area itself almost transversely or obliquely forward, though in a less degree than on the margin. In our species, the border of the area is nurrow and deeply grooved, and the linear ribs are obsolete, except near the beaks, where they are transverse, instead of having the marked obliquity seen in crenulata.

It differs from T. Emoryi, Con., in being more clongate, in having a much narrower corselet, and in other minor details. From T. Evansana, Meek, it can be distinguished by its less prominent base, more retreating anterior end, the greater number of its ribs, and by the entirely different direction of the lines on the corselet. 


\section{REMONDIA, N. Gen.}

Shell compressed, elongate subquadrate, closed at the extremitics (or perhaps slightly gaping posteriorly). Ligament very short, external. Hinge composed of three radiating cardinal teeth in cach valve, and a long posterior tooth in the left, with a corresponding tooth in the right. The middle cardinal of the left valve is transversely striate, as in Trigonia, and is slightly grooved on its face, the anterior is linear and smooth, and the posterior is also smooth, at least on its posterior facc. The posterior lateral, and its corresponding eavity, are irregularly rugosc. In the right valve, the anterior tooth is as large as the middle, the posterior is linear; further details unknown.

This genus is evidently elosely allied to Trigonia, its quadrate form not being unlike many of the speeiss of that genus, and the transversely striate teeth showing a marked resemblanee. I am unable to say, even with a largeseries before me, whether the posterior end was closed or not. In every ease the extremity is more or less broken, and from the appearanee of the most perfect specimens, it is not improbable that there may have been a slight gaping of the lower portion of the posterior margin.

In giving the above name to this genus, I take great pleasure in at least fulfilling a promise, made three or four years ago, of dedicating it to my old friend Auguste Rémond de Corbineau, who was eut off almost at the commencement of a career, that promised more than ordinarily brilliant results, and whose untimely death was mourned by all who knew him.

\section{R. FURCATA, n. s. \\ Pl. 36, Fig. 17, $17 a$.}

SHELl thick, clongatc, subquadrate, compressed; beaks small, promincnt, placed not quite a third of the length from the anterior end, which is narrowly rounded in the middle and retreats rapidly, nearly straight above, and convex below; cardinal margin very slightly concave, nearly parallel with the base; base faintly sinuous, broadly convex in advance, straight to concave posteriorly; posterior end concave in the middle, prolonged into two blunt 
points, the lower a little the longest. Adjoining the hinge line, both in advance of and behind the beaks, the surface is concavely truncated, making two plain areas with converging sides, bordered by sharp angles, corrcsponding in advance to a lunule, and posteriorly to the corselet of a Trigonia. A strongly marked umbonal ridge runs from the beaks to the posterior basal angle, above which the surface is concave, and in front of which it is very slightly convex. Surface marked by very irregular concentric ridges, less distinct towards the base, and corresponding in direction with the lines of growth.

Length (restored), 2.5 inches; width, 1.3 inch; diameter of two valves, .65 inch.

\section{CUCULLAEA, Lam.}

\section{C. inermis, n. s.}

Pl. 36, Fig. 18.

SHelu large, thick, subquadrate, very convex, variable in outline. Beaks largc, incurved, distant, variable in position, placed sometimes but slightly in advance of the middle, somctimes not more than a third of the length from the anterior end. Anterior end nearly straight above, rounding into the base bclow; posterior end very variable, more or less produced, sloping above, narrowly rounded below; base nearly straight or slightly convex; area broad, from two-thirds to three-fourths of the length of the shell; umbonal ridge rounded, posterior to which the surface is nearly flat, and almost at a right angle to the portion in advance. Surface marked only by lines of growth; in one unusually perfect specimen, ninute radiating lines can be detected near the beaks, by the aid of a glass.

Dimensions of three specimens: length, 3.0 inches; width, 2.2 inches; diameter of two valves, 2.0 inches. Length, 2.5 inches; width, 2.1 inches; diameter of two valves, 2.0 inches. Length, 1.9 inch; width, 1.5 inch; diameter of two valves, 1.3 inch.

This shell is not unlike $C$. truncata, of the California rocks, but is easily dis- 
tinguished by its more anterior beaks, more rounded umbones, shorter hinge, less prominent anterior end, and straighter base. It is larger than C. Nebraseensis, and its more square form will distinguish it.

\section{GRYPHAA, Lam.}

Perhaps no fossil in the Cretaceous formation of North America has been the subjeet of more discussion, or has been more misunderstood than Gryphaa Pitcherii. One of the prineipal reasons for this, is the fact that Dr. Morton described the speeies from a very small specimell, and gave, in his "Synopsis," but a single figure, which was not over eharacteristic. Dr. Roemer, after studying the fossils of Texas, visited the Museum of the Philadelphia Academy to study Morton's types, but did not suceed in finding the original specimen of this species, it having been earelessly thrown aside, in a drawer full of duplieates and worthless fragments, from which I had the good fortune to disinter it in 1860 or 1861, after years of eoncealment. Failing to obtain more reliable information than that furnished by Morton's short description and only passable figurc, Dr. Roemer applied the name to a form very common in Texas, but which, as will be seen below, I believe to be distinet from the true Pitcherii.

In Emory's report of the Mexican Boundary Survey, Mr. Conrad indicated a variety with strongly carinated and deflccted beaks, under the name of G. Pitcherii var. navia. This is now universally considered by American Palæontologists as a distinet species.

Professor Mareou, having advanced the theory of the Jurassic age of a large portion of the roeks of the Southwest, and being inclined to bolster it up by foreing evidence to its support, in 1855, in the Bulletin of the Geological Society of France, and again in his Geology of North America, seleeted the "variety" navia as Morton's, so ealled, typical form, and said the true Pitcherii was merely a variety of the Jurassic dilatata, calling it the "variety Tucumcarii." In the latter publication he gives excellent figures of navia under the name of Pitcherii, and illustrates both the more common and the most dilated form of the true Pitcherii, under the name of "var. Tucumcarii." My attention has been eonstantly directed to the question for the last eight or nine years, and $I$ have made it a point to study eritically, all the reputed speeimens of Pitcherii, and of the allicd forms that have come within my reach, and have arrived at the conelusion that there have been three well-marked and distinet species included under this name, as follows:

\section{G. Pitcherit, Morton.}

(Gryphaen Pitcherii, Morton; Synopsis, p. 55, pl. 15, fig. 9.)

(G. Pitcherii, Con.; Emory's Report, p. 155, pl. 21, fig. $3 a, b$ (exclus. c, $d$ ).)

(Id., Con. ; loc. cit., pl. 10, fig. 2.) 
(Ostrea vesicularis, d'Orb. (not Lam.); Prod. Pal. Strat., Vol. 2, p. 256, Etage 22, No. 925.)

(G. dilatata, var. Tucumcarii, Marcou; Bull. Soc. Géol. de France, Vol. xi, pl. 21, fig. 3.)

(Id., Marcou; Geol. N. A., p. 43, pl. 4, fig. 1, $a, b, 2,3$. )

(G. Pitcherii, Gabb; see remarks Proc. Phil. Acad., 1861, p. 22.)

SHell moderately thick, more or less rounded trigonal, expanded. Lower valve variably and unequally bilobate, the large lobe forming about two-thirds of the bulk of the valve, separated from the smaller lobe by a radiating depression, most strongly marked towards the base. Beaks small, incurved, or very slightly deflected towards the side of the small lobe; umbones large, prominent, and rounded; no umbonal ridge. Sides sloping nearly straight, or slightly convex, downwards from the hinge margin, unequal, the side of the small lobe being always the most prominent. Basal margin more or less strongly sinuous. Hinge triangular, short, broad, and straight. Upper valve nearly flat, or a little convex near its straightest margin, caused by a greater thickness at that point; rounded, trigonal to suborate, or almost subcircular. Surface smooth or sliglitly imbricated.

This description is based principally on Dr. Morton's original specimens, somewhat amplified by the assistance of additional material.

\section{G. Navia, Con.}

(Gryphaea Pitcherii, var. navia, Con.; Emory's Report, Mexican Boundary, p. 155, pl. 7 , fig. $3, c, l$ (cxclus. $a, b)$ ).

(G. Pitcherii, Marcou (not Morton); Geol. N. A., p. 38, pl. 4, fig. 5, a, b, fig. 6.)

(G. navia, Gabb ; Proc. Phil. Acad., 1861, p. 22.)

SheLL very thick, elongate, narrow subtrigonal. Lower valve thick, very indistinctly or not at all bilobate; beaks acute, prominent, flattened laterally, and deflected entirely beyond the left hand margin; umbones prominent, angulated, a sharp umbonal ridge running from the beak to the basal margin; sometimes a slightly marked, rounded ridge occupies the position of the smaller PAL. YOL. II. -36 
lobe of Pitcherii. Base entire; sides sloping from the hinge line to the base at nearly equal angles, the right side a little eonvex, the left about equally eoneave. Surfaee more or less squamose, sometimes a series of very rough imbrications on the umbonal ridge. Hinge long, narrow, and eurved laterally with the defleetion of the beak. Upper valve small, thick, narrow, flat, and oblique.

For the resemblances and differences between the three forms, see notes following the description of the succecding species.

\section{G. mucronata, Gabb.}

(G. Pitcherii, Roem. (not Morton) ; Kreid. Tex., p. 73, pl. 9, fig. 1.)

(G. Pitcherii, Schiel ? (not Morton) ; Pacific Railroad Report, Vol. 2, p. 108, pl. 3, fig. 9.)

Sheul long, narrow, subtrigonal, very thick. Lower valve strongly bicarinate by two radiating ridges, one running from the umbones, the other near the opposite margin, dividing the surface rudely into three portions, two marginal planes and an intermediate one, often eoneave. Beaks very prominent, produeed, median and strongly ineurved; umbones very prominent and rounded. Hinge long, narrow, and straight. Sides nearly equal, diverging equally at a narrow angle; base slightly convex, entire. Upper valve long, narrow, thick, and straight. Surface marked by subsquamose lines of growth.

The differences between these three species are as follows: $G$. Pitcherii is dilated and very distinctly bilobate, the beaks are small and incurved, and the umbones are broadly rounded. G. navia is narrow triangular, the beaks are deflected excessively, and the umbonal ridge is acute-angular, sometimes not being more than forty to forty-five degrees. The hinge is also deflected. $G$. mucronata is also narrow, but the beaks, which are very long and incurved, in some cases into a prominent hook, are placed exactly in the median line, and the umbone and umbonal ridges are rounded, never even subangular.

Mucronata and Pitcherii agree in the median beaks, but differ in form. Navia and mucronata have the narrow trigonal form, but differ in the size, shape, and positions of the beaks. There is less resemblance between these latter on the one side, and Pitcherii on the other, than there is between some of the varieties of 
Pitcherii and vesicularis. At the samc time the bcaks will distinguish them between themselves.

G. mucronata occur's abundantly among the Mcxican fossils.

\section{EXOGYRA, Say.}

\section{E. plicata, Lam., sp.}

Pl. 36, Fig. 19, $19 a$.

(Gryphaea plicata, Lam.; 1819, An. S. Vert., Vol. 6, p. 199.)

(Exogyra plicata, Goldf. ; Petr. Germ., Vol. 2, p. 37, pl. 87, fig. 5.)

(E. plicata, Roem.; Kreid., p. 48.)

(E. flabellata, Goldf.; Petr. Germ., Vol. 2, p. 38, pl. 87, fig. 6 (young) ).

(Ostrea flabellata, d'Orb. ; Pal. Fr., Vol. 3, p. 717, pl. 475.)

(E. harpa, Goldf.; Petr. Germ., Vol. 2, p. 38, pl. 87, fig. 7.)

(Gryphoea harpa, Fbs.; Quart. Jour. Gcol. Soc., 1844, p. 250, pl. 3, fig. 12.)

(Ostrea Matheroniana, d'Orb; Pal. Fr., Vol. 3, p. 737, pl. 485.)

(E. Matheroniana, Con.; Emory's Rep., p. 154, pl. 8, fig. 11.)

(E. Boussingaultii, d'Orb. ; Foss. de Col., p. 57, pl. 3, fig. 10 ; pl. 5, fig. 8, 9.)

(E. Boussingaultii, d'Orb.; Am. Merid., p. 91, pl. 18, fig. 20.)

(Ostrea Boussingaultii, d'Orb. ; Pal. Fr., Vol. 3, p. 702, pl. 468.)

(E. subplicata, Roem.; Ool. Nach., tab. 18, fig. 17.)

(E. spinosa, Math.; Cat., p. 192, pl. 32, fig. 6, 7.)

(E. Texana, Roein.; Kreid. Tex., p. 69, pl. 10, fig. $1 a, b, c, d$. )

(E. Texana, Shum.; Marcy's Rep., Red. R., p. 205, pl. 5, fig. 1, 2.)

Quite common in Texas and at Arivechi, as well as at all or most of the intermediate localities. I cannot believe that, in an oyster, the number or size of the plications, within reasonable limits, can be depended upon as of specific value, and if is on these alone that the above long list of names has bcen proposed.

\section{OSTREA, Linn.}

\section{O. SP. INDET.}

A small, thin oyster occurs, incrusting many of the larger fossils, and growing in clusters. It is rarely more than an inch or an inch and a quarter long, is closely attached by the whole lower surface, and, where not crowded out of shapc, is irregularly trigonal, a little oblique, and narrows regularly towards the beaks. 


\author{
RADIATA. \\ PYRINA, Desmoul. \\ P. Parryi, Hall. \\ (Pyrina Parryi, Hall; Emory's Rep., p. 144, pl. 1, fig. 1 a-d.)
}

Rare; but three or four specimens were found.

\title{
CYPHOSOMA, Agas. \\ C. Texanum, Roem.
}

(Diadema Texana, F. Roem.; Texas, p. 392.)

(Cyphosoma Texanum, Roem.; Kreid. Tex., p. 82, pl. 10, fig. 6.)

(Id., Hall; Emory's Report, p. 145, pl. 1, fig. 3 a-c.)

More common than the preceding.

\section{TURBINOLIA, Lam.}

\section{? T. Texana, Con.}

(Turbinolia Texana, Con.; Emory's Report, p. 144, pl. 2, fig. 3 a, b.)

Very common.

\section{UNDETERMINED.}

Two or three specics of incrusting tubes occur in the collection, perhaps all Serpulidee. They are all, with one exception, so weathered that all distinctive characters arc oblitcrated. The exceptional case is the one of which a somewhat magnified view is given on Pl. 36, Fig. 11. It may be a Serpula, or not impossibly one of the Vermetidce. It is closely attached by its lower surface, which is expanded laterally into a subsquamose plate; the upper surface is longitudinally convex; with a slight ridgc or offset on each side, and is transversely striate or rugosc. The aperture is transversely subelliptical in section. This form is very rare. I have seen but a single specimen. 


\section{INDEX OF GENERA AND SPECIES.}

AcIla, 102, 197, 250.

Castrensis, 102.

truncata, 197, 250.

ACMÆA, 51, 86, 172, 230.

marmorea, 87.

mamillata, 87 .

mitra, 87.

patina, 52, 86.

pelta, 52.

persona, 52, 86 .

rudis, 51 .

scabra, 86.

scurra, 87.

spectrum, 52, 86.

Tejonensis, 172, 230.

Traskii, 173.

Acrosmilia, 253.

striata, 253.

AстжоN, 115, 232.

ellipticus, 115.

impressus, 232.

Act aonella, 173, 232.

oviformis, 173, 232.

ACTAONINA, 173, 231.

Californica, 231.

pupoides, 173, 231.
Agasoma, 46.

gravida, 46, 72 .

sinuata, 46, 72 .

Akera, 88.

culcitella, 88.

Alia, 10,76 .

Richthofeni, 10, 76.

A Mauropsis, 223 .

alveata, 223.

oviformis, 223.

Amiantis, 96.

callosa, 56, 96.

Ammonites, 130, 210, 258.

Archiacianus, 136.

Batesii, 132, 211.

Brewerii, 130, 210.

Chicoensis, 211.

complexus, 133, 211.

Cooperii, 212.

fiaternús, 137, 212.

Haydeni, 210.

Hoffmannii, 131, 137, 211.

jugalis, 133, 212.

Michelinianus, 136.

Newberryanus, 210, 212.

Pederualis, 258. 
Ammonites Peruvianus, 210.

pleurisepta, 258.

ramosus, 210.

Rémondii, 211.

Stoliezkanus, 135, 212.

subtricarinatus, 132, 211.

Suciaensis, 133, $2 \mathrm{L1}$.

Tehamaensis, 132, 211.

'Traskii, 131, 137, 210.

tuberculatus, 136.

Vancouverensis, 211.

Whitneyi, 134, 212.

Amphidesma, 94.

decisa, 94.

pulchra, 94.

Amphissa, 74 .

corrugata, 74 .

Ampullina, 161, 223.

alveata, 160, 223.

striata, 161, 223.

Anycla, 76.

carinata, 76 .

gausapata, 76 .

Anatina, 177, 234.

inequilateralis, 234.

lata, 234.

quadrata, 177, 234.

Tryoniana, 234.

Anchurs, 165, 226, 262.

angulata, 226.

Californica, 226.

earinifera, 166, 226 .

exilis, 226.

filciformis, 165,226 .

monilifera, 262 .

transversa, 165, 226.

Ancillaria, 9, 76, 220.
Ancillaria elongata, 220.

Fishii, 9, 76.

Ancyloceras, 138, 213.

lineatus, 139, 213.

percostatus, 138, 213.

quadratus, 213.

Rémondii, 138, 213.

Angaria, 229, 264.

cingulata, 264.

ornatissima, 229

Angulus, 93.

Gouldii, 93.

Anisomyon, 231.

Meekii, 231.

Anomalocardia, 121.

devincta, 121.

A Nomia, 105, 202, 252.

lampe, 106.

lineata, 202, 252.

subeostata, 105.

Vancouverensis, 202, 252.

Anthonya, 244.

eultriformis, 244.

Aporrhais, 165, 226.

angulata, 226.

Californica, 226.

exilis, 226.

falciformis, 165, 226.

Arca, 31, 121, 248.

Breweriana, 249.

eongesta, 121.

decurtata, 248.

devincta, 121.

equilateralis, 249.

gravida, 248.

Hornii, 249.

mierodonta, 121. 
Arca Obispoana, 121.

sulcicosta, 31, 124.

Vancouverensis, 249.

ArchitectonicA, 224.

cognata, 224.

Hornii, 224.

inornata, 224.

Veatchii, 224.

Arсомy , 179, 235.

undulata, 179, 235.

Arcopagia, 92, 118.

medialis, 92.

unda, 118.

Artemis, 97.

ponderosa, 97.

Asaphis, 181, 236.

multicostata, 181, 236.

undulata, 236.

Astarte, 244.

Conradiana, 244.

Mathewsonii, 244.

Tuscana, 244.

Asterias, 37, 110.

Rémondii, 37, 110.

Astrocenia, 254.

petrosa, 254.

Astrodapsis, 37, 110.

Antisellii, 110.

tumidus, 37, 110.

Whitneyi, 37, 110.

Ataphrus, 171, 229.

crassus, 171, 229.

Atractus, 146.

Atresius, 168, 227.

liratus, 169, 227.

Atdria, 69, 210.

Mathewsonii, 210.
Aturia Vanuxemi, 69. ziczac, 69.

Aucella, 194, 247.

Piochii, 194, 247.

Avellana, 264.

A vicula, 246.

pellucida, 246.

AxINÆA, 102, 196, 249.

Barbarensis, 102.

cor, 250.

patula, 102.

sagittata, 196, 249.

Veatchii, 249.

Baculites, 145, 214.

Chicoensis, 214.

inornatus, 214.

occiden talis, 145, 214.

ovatus, 145, 214.

Balanus, 107.

Estrellanus, 61, 107.

Barbatia, 249.

Morsei, 249.

BeLA, 152, 218. clathrata, 152, 218.

BeLennites, 129, 209. impressus, 129, 209.

Bittium, 12, 79.

armillatum, 79.

asperum, 12, 79 .

rugatum, 79 .

Brachysphingus, 155, 219.

liratus, 156, 219.

sinuatus, 156, 219.

Buccinanops, 157.

moniliferum, 157. 
Buccinum, 74, 112, 156, 219.

corrugatum, 74.

devinctum, 112.

elegans, 74 .

fossatum, 47.

liratum, 156, 219.

Bulda, 87, 115, 116, 232.

Adamsi, 87.

culcitella, 88.

Hornii, 232.

jugularis, 116.

nebulosa, 87.

petrosa, 115.

Bullia, 156, 219.

moniliferum, 157.

striata, 157, 219.

Busycon, 111, 149, 217.

Blakei, 149.

Oregonensis, 111.

CAEUM, 81.

Cooperii, 81.

Cæsia, 47.

Caldianassa, 111, 127, 209.

Oregonensis, 111.

Stimpsonii, 127, 209.

Calliostoma, 17, 83, 170, 229.

annulatum, 83.

canaliculatum, 83.

costatum, 83 .

radiatum, 170, 229.

tricolor, 17, 83 .

Callista, 24, 96.

Voyi, 24, 96.

Calyptræa, 81.

spinosa, 81.
Cancellaria, 11, 50, 79, 114.

altispira, 50, 79 .

gracilior, 50, 79 .

Oregonensis, 114.

tritonidea, 11, 79 .

vetusta, 12, 79 .

Cancer, 1, 69.

Brewerii, 1, 69.

Cantharus, 147.

Cardita, 100, 187, 243, 268.

alticosta, 268.

IIornii, 187, 243.

monilicosta, 101.

occidentalis, 101.

planicosta, 187, 243.

subtenta, 101.

veneriformis, 243.

ventricosa, 100.

CARDIU M, 27, 98, 120, 187, 242, 266.

annulatum, 187, 242.

Brewerii, 242.

Californianum, 98.

centifilosum, 99.

Cooperii, 242.

corbis, 98 .

elatum, 99.

Gabbii, 21, 92.

granuliferum, 267.

linteum, 242.

Meekianum, 27, 99.

modestum, 120.

Nuttallii, 98.

Panamense, 99.

Placerensis, 187, 242.

Rémondianum, 242.

sabulosum, 267.

scitulum, 242. 
Cardium substriatum, 99. translucidum, 187, 242.

Caryatis, 56, 95, 185, 240.

Barbarensis, 56, 95. nitida, 186, 240.

Cassidea, 113.

bilirata, 113 .

petrosa, 113.

Catinus, 50, 114, 223.

obliquus, 223.

scopulosus, 114.

Cellepora, 107.

Bellerophon, 107.

Californiensis, 107.

Cemoria, 115.

crucibuliformis, 115 .

Cerithidea, 79.

Californica, 79 .

sacrata, 79 .

Cerithiopsis, 114, 224.

alternata, 224 .

Oregonensis, 114.

Cerithiu m, 79, 112, 263.

Californicum, 79.

mediale, 112.

sacratum, 79 .

Mexicanum, 263.

Cheninitzia, 114, 162, 223, 260.

papillosa, 114.

planulata, 162, 223.

Spillmanni, 162, 223.

Texana, 261.

zebra, 260.

Chione, 23, 55, 94, 118, 239.

angulata, 239.

angustifrons, 118.

Lordii, 96.
Cbione Mathewsonii, 23, 95.

Oregona, 95.

pertenuis, 55, 95 .

succincta, 94 .

varians, 239.

vespertina, 118.

Whitneyi, 23, 95.

Chiton, 87.

Stellcrii, 87.

amiculatus, 87.

Californicus, 87.

chlamys, 87.

Sitkensis, 87.

Chlorostoma, 84.

brunneum, 84 .

funcbrale, 84 .

Pfeifferii, 84.

Chrysodomus, 3, 71.

tabulatus, 71 .

Cinulia, 231, 264.

Mathewsonii, 231.

obliqua, 231.

pinguis, 231.

reetilabrum, 264.

Clatiuurelia, 7, 73.

Con radiana, 7, 73.

Clavatula. 215.

Californica, 215.

Clavella, 4,46 .

gravida, 4, 46, 72.

sinuata, 5, 46, 72 .

Clidophora, 54, 90.

punctata, 54, 90.

Cuisocolus, 188, 243.

dubius, 189, 243.

Clypeaster, 36, 109.

Gabbii, 36, 109.

PAL. VOL. II. -37 
Cly pidelua, 86, 124.

Vimaculata, 86, 124.

callomarginata, 86, 124.

Columbella, 10, 76 .

carinata, 76.

gausapata, 76.

Richthofeni, 10, 76 .

Colus, 111.

arctatus, 111.

Cominella, 74.

corrugata, 74.

Conchocere, 27, 99.

bisecta, $27,99$.

disjuneta, 28,99 .

Conus, 78, 150, 225.

Californieus, 78.

Hornii, 225.

ravus, 78.

Rémondii, 225.

sinuatus, $150,217$.

Corbula, 116, 176, 233.

alæformis, 177, 234.

cultriformis, 234.

Diegoana, 116.

Evansana, 117.

Hornii, 176, 234.

parilis, 234.

primorsa, 233.

Traskii, 234.

Cordiera, 153, 218.

microptygma, 218.

mitræformis, 153, 218.

Crassatella, 29, 100, 189, 244.

alta, 189, 244.

eollina, 29, 100.

compacta, 190, 244.

grandis, 189, 244.
Crassatella Uvasana, 244.

Crenella, 246.

concentrica, 246.

Crepidula, 81.

adunca, 82.

dorsata, 82.

lingulata, 82 .

navicelloides, 82.

prarupta, 81.

rostriformis, 82.

Crioceras, 138.

latus, 213.

percostatus, 138, 213.

Rémondii, 138, 213.

Crucibulum, 81.

spinosum, 81 .

Crypta, 81, 115, 228.

adunca, 82 .

dorsata, 82.

grandis, 81.

lingulata, 82.

navicelloides, 82 .

pileum, 228.

prarupta, 81.

princeps, 82.

rostralis, 82, 115.

rostriformis, 82, 115.

Cryptochiton, 87.

Stellerii, 87.

Cryptomya, 90, 116.

Californica, 90, 116.

ovalis, 90.

Cucullas, 249, 271.

equilateralis, 249.

inermis, 271.

Matbewsonii, 249.

truncata, 249. 
Cuma, 9, 75 .

biplicata, 9, 75 .

Cumingia, 94.

Californica, 94.

Cyclas, 100, 120.

acutilineatus, 100.

Estrellana, 120.

fibrosa, 120.

permacra, 120.

Crlichna, 115, 232.

costata, 232.

Oregona, 115.

petrosa, 116.

Cylindrites, 232.

brevis, 232.

Cymbophora, 179, 236.

Ashburnerii, 180, 236.

Cyphosoma, 276.

Texanum, 276.

Cypraa, 78, 163, 225.

Bayerquei, 163, 225.

Mathewsonii, 164, 225.

spadicea, 78 .

Cyprina, 99.

bisecta, 99 .

Cyprinella, 242.

tenuis, 242.

Crrena, 26, 98.

Californica, 98.

Cytherea, 95, 96, 118.

callosa, 96.

crassatelloides, 96.

gigantea, 97 .

Oregona, 95.

solidissima, 96 .

vespertina, 118.

Defrancia, 7 .
Delphinula, 264.

Dentalium, 86, 115, 230.

Cooperii, 230.

hexagonum, 86.

Nanaimoensis, 230.

pusillum, 230.

stramineum, 230.

substriatum, 115.

Diadema, 276.

Texana, 276.

Diadora, 115.

crucibuliformis, 115.

Drodus, 242.

tenuis, 212.

Dione, 95, 118, 239, 241.

angustifrons, 118.

brevilineata, 95.

Conradiana, 241.

Oregona, 95.

Uvasan: 239.

varians. 239.

vespertina, 118.

Diptychoceras, 143, 214.

lævis, 144, 214.

Disconelix, 224.

Leana, 224.

Doliopsis, 113.

biliratus, 113.

petrosus, 113.

Dolium, 113.

petrosum, 113.

Donax, 89, 96, 183, 238.

latus, 183, 238.

protexta, 89 .

stultorum, 96.

Dosinia, 24, 96, 97, 119, 240.

alta, 25, 97, 240. 
Dosinia callosa, 96.

Conradi, 25, 97.

Dunckerii, 57.

clevata, 240.

gyrata, 241.

inflata, 241.

longula, 119.

Mathewsonii, 57, 97.

montana, 119.

pertenuis, 241.

ponderosa, 57, 97.

Staleyi, 24, 57, 97.

subobliqua, 119.

tenuis, 240.

Dosiniopsis, 240.

alta, 240.

Drillia, 72.

inermis, 72.

perversa, 73 .

Voyi, 7, 73.

Ecimnaracinnius, 36, 109.

Brewerianus, 36, 109.

Ellipsosmilia, 254.

granulifera, 254.

Emarginula, 230.

radiata, 230.

Ensis, 116.

curtus, 116.

Entalophora, 109.

punctulata, 109.

Epona, 164, 225.

Nathewsonii, 164, 225.

Eripacina, 148, 216.

Hoffmannii, 149, 216.

perforata, 149, 216.
Eripachya ponderosa, 149, 216.

Eriphy LA, 244.

umbonata, 244.

Euclia, 10, 79.

tritonidea, 11, 79.

Eusprat, 160, 223, 260.

alveata, 160, 223.

tabulata, 260 .

ExogYra, 253, 275.

Boussingaultii, 275.

flabellatı, 275.

harpa, 275.

Matheroniana, 275.

parasitiea, 253.

plicata, 275.

spinosa, 275.

subspinosa, 275 .

Texana, 275.

Fasciolaria, 220.

Io, 220 .

læviuscula, 220.

sinuata, 220.

Ficopsis, 11:, 158, 221.

Cooperii, 159, 221.

IIoriii, 158, 221.

modesta, 113.

Rémondii, 158, 221.

Ficus, 48, 77, 113, 221.

cypraoides, 160, 221.

Dussumieri, 48.

mamillatus, 221.

modestus, 113.

nodiferus, 48,77

Ocoyanus, 113.

pyriformis, 48,77 . 
Fissurella, 86. aspera, 85. crenulata, 85 . volcano, 86 .

F'LABELlum, 253.

Rémondianum, 253.

Fusus, 70, 73, 111, 145, 214, 259. ambustus, 71 .

aratus, 215.

arctatus, 111.

Averillii, 215.

Barbarensis, 111.

Californicus, 215.

cancellatus, 73.

Cooperii, 159, 221.

corpulentus, 112.

Diaboli, 215.

flexuosus, 215.

geniculus, 71 .

Hornii, 158, 221.

Kingii, 215.

Martinez, 146, 214.

Mathewsonii, 215.

Mexicanus, 259.

occidentalis, 146, 215.

Oregonensis, 73, 111.

Orpheus, 70.

Rémondii, 158, 221.

robustus, 111.

rugosus, 111.

tumidus, 145, 215.

Gadus, 230.

pusillus, 230 .

Galeodea, 112.

petrosa, 112.
Galerites, 123.

Oregonensis, 123.

Galerus, 228.

cxcentricus, 228.

GARI, 21, 92, 237.

alata, 21, 92.

texta, 237 .

Gloobiconcha, 232.

liémondii, 232.

Gizchmeris, 89.

abrupta, 90.

Estrellanus, 90.

generosa, 89.

Gryphis, 85.

aspera, 85 .

Goniomya, 235.

borealis, 235 .

Granocardium, 266.

sabulosum, 267.

GryPh $2 A$, 253, 272, $27 \mathrm{~s}$.

dilatata, 272.

harpa, 275.

mucronata, 274 .

navia, $273,274$.

Pitcherii, 272, 273, 274.

plicata, 275.

Tucumcarii, 272.

vesicularis, 253, 274.

Gymnarus, 163, 225.

manubriatus, 163, 22e.

Gyrodes, 222.

Conradiana, 222.

expansa, 222.

Haliotis, 85.

rufescens, 85 . 
IIA Mites, 139, 212. annulifer, 143. quadratus, 139, 213. Vancouverensis, 212.

Haydenia, 219. impressa, 219.

Helcion, 230. circularis, 230. dichotoma, 230.

Helicancylus, 140, 214. xquicostatus, 141, 214.

HeLiCA ULAX, 166, 226. bicarinata, 166, 226. costata, 167, 2:6.

Helicoceras, 212. vermicularis, 212. Brewerii, 213. declive, 213.

HeLIX 88. facta, 88 .

Hillebrandi, 88. Mormonum, 88. 'Tryonii, 88. tudiculata, 88 .

Hemifusus, 158, 221. Cooperii, 159, 221. Hornii, 158, 221. Rémondii, 158, 221.

Hemiactra, 19, 5 400. lenticularis, 19, 90. oecidentalis, 54, 91 .

Here, 29, 100.

Richthofeni, 100.

IEteroterma, 151, 217. troehoidea, 151, 217.

Hinnites, 105. crassa, 105.

Hinnites giganteus, 105.

Poulsoni, 105.

Hipponyx, 82.

Grayanus, 82.

Hippurites, 63.

Honomya, 179, 236. coneentriea, 179, 236.

Hypogella, 89, 175, 233. cuneatus, 175, 233.

Diegoensis, 176, 233. protexta, 89 .

IDMONEA, 109.

Californiea, 109.

Inoceranius, 193, 246.

Elliotii, 193, 246.

Piochii, 191, 194, 245, 247.

subundatus, $2+7$.

Whitneyi, 193, 246.

JANIRA, 104.

bella, 105.

dentata, 104.

LiACUNA, 80.

carinata, 80 .

solidula, 80.

Lævicardium, 99, 187, 242.

annulatum, 187, 242.

elatum, 99.

linteum, 242.

substriatum, 99.

LEDA, 103, 121, 197, 250. cælata, 103. 
Leda cuneata, 103.

Gabbii, 197, 25 .

Oregona, 121 .

penita, 121.

protexta, 197, 250.

translucida, 250.

Willamettensis, 122.

Leptonyx, 85.

bacula, 85 .

sanguinea, 85.

LEPTothyra, 85.

bacula, 85 .

sangruinea, 85.

Lichenopora, 109.

Californica, 109.

Lima, 201, 251.

appressa, 201, 251.

microtis, 201, 202, 251.

multiradiata, 201, 252.

Shastaensis, 251.

LIMOPSIS, 121, 192, 249.

nitens, 121.

Oregonensis, 121.

sagittata, 196, 249.

transversa, 250.

Liocardium, 99.

elatum, 99.

substriatum, 99.

Liocium, 174, 232.

punctatum, 174, 232.

Lioconcha, 96.

Neweombiana, 96 .

Liropecten, 105.

crassical'do, 105.

Estrellanus, 105.

Veatchii, 105.

volæformis, 105.
Litilasia, 13, 80.

antiqua, 13, 80 .

Lithoph $\boldsymbol{A g U s ,} 246$.

oviformis, 246.

Lit'torina, 14, 80, 227.

compacta, 227.

patula, 80.

Pedroana, 80.

planaxis, 80 .

Rémondii, 14, 80.

Loripes, 100, 189, 243.

dubius, 189, 243.

parilis, 100 .

Lottia, 52, 86.

gigantea, 52.

pallida, 87.

scabra, 86 .

spectrum, 86 .

Loxotrema, 168, 227.

turrita, 168, 227.

LUCAPINA, 85.

crenulata, 85.

Lucina, 28, 100, 120, 189, 243.

acutilineata, 100.

borealis, 100 .

Californica, 100.

eretacea, 243.

cumulata, 243.

Estrellana, 120.

fibrosa, 120.

gyrata, 241.

nasuta, 189, 243.

Nuttallii, 100.

permacra, 120.

postice-radiata, 189, 243.

postradiata, 189, 243.

Richthofeni, 29, 100. 
Lucina subcircularis, 243. tetrica, 100.

Lunatia, 77, 114, 222, 259. avellana, 222.

Conradiana, 222.

Hornii, 222.

Lewisii, 77.

nueiformis, 222 .

Oregonensis, 114.

Pedernalis, 259.

Shumardiana, 222 .

Luponia, 78, 163, 225.

Bayerquei, 163, 225.

spadicea, 78.

Lutraria, 91, 95, 117, 236.

capax, 91.

maxima, 91.

Nuttallii, 91.

transmontana, 117.

Trastii, 56, 95.

truncata, 286.

Lutricola, 92.

alta, 92.

viridotineta, 92 .

Lysis, 229.

duplieosta, 229.

MaCh Ra, 89.

patula, 89 .

Macoma, 93, 118.

arctata, 118.

calcarea, 124.

edulis, 93 .

expansa, 93.

inconspicua, 94, 124.

inquinata, 93.
Macoma nasuta, 93.

Oeoyana, 118.

Pedroana, 94, 124.

yoldiformis, 93.

Mactra, 91, 117, 179, 236.

albaria, 91.

Ashburnerii, 180, 236.

Diegoana, 91.

falcata, 92.

Gabiotensis, 117.

Gibbsana, 236.

maxima, 91.

planulata, 91.

tenuissima, 179, 236.

Mangelia, 73.

variegata, 73.

Margarita, 85.

acuticostata, 85 .

salmonca, 85, 123.

Margaritella, 172, 229.

angulata, 172, 229.

erenulata, 229.

globosa, 229.

Marginella, 76.

Jewettii, 76 .

Martesia, 175, 233.

clausa, 175, 233.

Meeis ia, 248.

navis, 248.

radiata, 248 .

sella, 248.

Megistostoma, 232.

striata, 232.

Melania, 13, 80.

Taylori, 13, 80.

Meleagrina, 192, 246.

antiqua, 192, 246. 
Membranipora, 108.

Barbarensis, 109.

Californica, 108.

Mercenaria, 22, 55, 94, 239.

Kennerleyi, 22, 55, 94.

perlaminosa, 22, 55, 94 .

varians, 239.

Meretrix, 56, 118, 185, 239.

arata, 240.

Californica, 240.

decisa, 118.

fragilis, 185, 240.

Hornii, 185, 239.

lens, 239.

longa, 240.

nitida, 186, 240.

ovalis, 240.

Traskii, 56, 95.

Tularana, 119.

uniomeris, 118.

Uvasana, 239.

Mesalia, 114.

arenicola, 114.

Metula, $3,7 \cdot$.

Rémondii, 3, 72.

Mitra, 158, 221.

cretacea, 158, 221 .

Modelia, 80.

striata, 80.

Modiola, 30, 101, 120, 191, 245.

capax, 101.

contracta, 120.

cylindrica, 245.

flabellata, 101.

major, 191, 246.

multiradiata, $30,101$.

ornata, 245.

PAL. VOL. II. -38
Modiola recta, 101.

Siskiyouensis, 245.

Molopophorus, 156, 219.

striata, 157, 219.

Monoceras, 75.

engonatum, 75 .

lugubris, 75 .

unicarinatum, 75 .

Morio, 223.

tubereulatus, 223.

Morrisia, 36, 124.

Hornii, 36, 124.

Mulinea, 19, 20, 91.

densata, 20.

densata, 19, 91.

Murex, 69, 111.

Californicus, 69.

festivus, 70.

fragilis, 111.

multicostatus, 70 .

Muricidea, 43, 69.

Barbarensis, 69.

Californica, 69.

paucivarieata, 43,69 .

My a, 90, 116.

abrupta, 90 .

Montereyana, 116.

subsinuata, 116.

Mrsia, 100, 243.

parilis, 100 .

polita, 243.

Mytilimeria, 90.

Nuttallii, 90.

Mrtilus, 30, 101, 120, 191, 245.

aseia, 191, 245.

bifurcatus, 101.

Californianus, 101. 
Mytilus humerus, 245.

Inezensis, 120.

Mathewsonii, 30, 101.

pauperculus, 245.

Pedroanus, 120.

quadratus, 191, 245.

MyUrella, 78.

simplex, 78.

Nacella, 87.

insessa, 87.

Narica, 115.

Diegoana, 115.

NAsSa, 47, 74, 219.

antiquata, 219.

Cooperii, 47, 74.

cretacea, 219.

fossata, 74 .

mendica, 47,74 .

perpinguis, 47,75 .

tegula, 74 .

Natica, $7 \bar{\imath}, 113,221,259$.

alvcata, 160, 223.

clausa, 77 .

genieulata, 113.

Herculea, 77.

Inezana, 77.

Lewoisii, 77.

Ocoyana, 77.

Pedernalis, 259.

Recluziana, 77.

saxea, 77.

Uvasana, 221.

Naticina, 223.

obliqua, 223.

Nautilus, 209.

Nautilus angustatus, 69.

Campbelli, 210.

Texanus, 209.

ziczac, 69 .

NEARA, 236.

dolabræformis, 236.

NeILo, 121, 122.

abrupta, 122.

Oregona, 121.

penita, 121.

Willamettensis, 122.

Neithea, 200, 251.

atava, 200.

duplicicosta, 200.

grandicosta, 200, 251.

Neptunea, 3, 44, 71, 146, 216.

altispira, 44, 71 .

cretaeca, 146, 216.

decemcostata, 45, 123.

gracilis, 149, 216.

Hoffmannii, 149, 216.

humerosa, 45, 71 .

lirata, 45, 123.

mueronata, 147, 216.

perforata, 149, 216.

ponderosa, 149, 216.

reeurva, 3,71 .

supraplicata, 149, 216.

tabulata, 71 .

Nerinea, 231.

dispar, 231.

Nerita, 170, 228.

euneata, 229.

deformis, 228.

triangulata, 229.

Neverita, 10, 77, 161, 222.

callosa, 10, 77 . 
Neverita globosa, 161, 222.

Recluziana, 77.

saxea, 77 .

secta, 222.

Niso, 224.

polita, 224.

Nucula, 102, 122, 197, 250.

abrupta, 122.

decisa, 102.

divaricata, 102.

Castrensis, 102.

Conradi, 102.

calata, 102.

impressa, 58, 102.

solitaria, 197, 250.

Traskana, 250.

truncata, 197, 250.

Nuculana, 121.

Oregona, 121.

penita, 121.

Willamettensis, 122.

OCINebra, 70.

interfossa, 70.

lurida, 70 .

Olivella, 75, 220.

biplicata, 75 .

botica, 75 .

Mathewsonii, 220.

Pedroana, 75.

Opalia, 78, 223.

crenatoides, 78 .

var. insculpta, 78 .

Matñcusonii, 223.

Ostrea, 60, 106, 123, 203, 252, 272. appressa, 203, 252.
Ostrea Attwoodii, 33, 106.

Bourgeoisii, 33, 106.

Brewerii, 252.

Boussingaultii, 275.

Cerrosensis, 35, 106.

flabellata, 275.

gallus, 106.

Heermannii, 107.

Idriaensis, 203, 252.

lurida, 106.

malleiformis, 252.

Panzana, 123.

subjecta, 123.

Tayloriana, 34, 106.

Titan, 107.

Veatchii, 34, 60, 106.

vesicularis, 253, 272.

vespertina, 107.

PaChydesma, 96, 119.

crassatelloides, 96.

Inczana, 119.

Pachypoma, 15, 83.

biangulatum, 15, 83 .

gibberosum, 83 .

Palmatractus, 147, 216

crassus, 148, 216.

Pallium, 105.

crassicardo, 105.

Estrellanum, 105.

Pandor.a, 18, 90, 117.

- bilirata, 117.

punctata, 54, 90.

scapha, 18, 90 .

Panopœa, 179, 236.

concentrica, 179, 236 .

Parapholas, 88. 
Parapholas penita, 88.

Patella, 86, 87, 230. insessa, 87.

scabra, 86 .

scurra, 87.

spectrum, 86 .

Traskii, 230.

Pecten, 32, 59, 86, 103, 122, 198, 250.

altiplicatus, 104 .

Californicus, 200, 251.

complexicosta, 199, 251 .

catilliformis, 103.

Cerrosensis, 32, 103

Coosensis, 122.

dentatus, 104.

desertii, 122 .

diseus, 122.

excavatus, 103.

Fabricii, 104.

hastatus, 104 .

hericeus, 104.

Icelandicus, 104.

interradiatus, 199, 251.

Islandicus, 104.

magnolia, 122 .

Martinezensis, 198, 251.

Meekii, 122.

Nevadanus, 12:.

operculiformis, 200, 251.

Pabloensis, 103.

Pealii, 104.

Peekhami, 60, 104.

Pedroanus, 60, 104.

propatulus, 103, 122.

Traskii, 198, 250.

tumidus, 104.

Peeten Veatchii, 32, 105.

ventricosus, 104.

Pectunculus, 98, 102, 121.

corbis, 98.

nitens, 121.

patulus, 100, 102.

Pelagus, 69.

Tanuxemi, 69.

Penitella, 88.

Conradi, 88.

penita, 88 .

spleea, 88.

Perissolax, 149, 217.

Blakei, 149, 217.

brevirostris, 217.

Gabbii, 150.

Perna, 120.

montana, 120 .

Petricola, 119.

Pedroana, 119.

Pitarella, 233.

alta, 233.

Phasianella, 82, 232.

compta, 82 .

perforata, 82.

Rémondii, 232.

Phidolopora, 108.

labiata, 108.

Pholadomya, 178, 235, 265.

borealis, 235.

Brewerii, 235.

nasuta, 235.

Oregonensis, 178, 235.

Sonorensis, 265.

subelongata, 178, 235 .

Pholas, 88.

penita, 88. 
Phyllonotus, 43, 69 .

paucivaricatus, 43, 49.

Pinna, 195, 247, 268.

Brewerii, 195, 247, 268.

indet, 268.

Pisania, 71.

forlis, 71 .

Plagiostoma, 60, 104.

annulata, 60, 104.

Pedroana, 60, 104.

truncata, 60, 104.

Plectosolen, 89, 116, 176, 233. curtus, 116.

Diegoensis, 176, 233.

parallelus, 233.

protexta, 89.

Pleuromya, 178, 235.

papyracea, 178, 235 .

Pleurotola, 5, 47, 112.

Carpenteriana, 5, 72

inermis, 72.

perversa, 6, 73 .

transmontana, 112.

Tryoniana, 6, 47, 72 .

Voyi, $7,73$.

Plicatula, 252.

variata, 252.

Polyplex, 7 .

gracilis, 70 .

Pomaulax, 83.

undosus, 83 .

Potamides, 227.

diadema, 227.

tenuis, 227.

Potamis, 79.

pullatus, 79.

Priene, 73.
Priene Oregonensis, 73.

Priscofusus, 71, 112.

corpulentus, 112.

devinetus, 112.

geniculus, 71 .

medialis, 112.

nodiferus, 112.

Oregonensis, 112.

Protocardium, 187, 24:, 267. granuliferum, 267.

Placerensis, 242.

translucidum, 187, 242.

Psammocola, 21, 92.

alata, 21, 92.

Psephis, 96.

Lordii, 96.

tantillus, 96.

Pseudocardium, 20, 55, 92.

Gabbii, 21, 92.

Pseudoliva, 219.

lineata, 219.

volutæformis, 220.

Pteronotus, 70.

festivus, 70 .

Ptiloteuthis, 128, 209.

foliatus, 128, 209.

Prychoceras, 142, 143, 214.

aquicostatus, 142, 214.

Dupinianum, 143.

Forbesianum, 143, 144.

Ptychostylis, $16,84$.

eaffea, 16,84 .

Pugneluus, 162, 225.

contortus, 163.

hamulus, 162, 225.

manubriatus, 163, 225.

Purlura, 75, 112. 
Purpura attenuata, 75.

canaliculata, 75 .

marginata, 75 .

lapillus, 75 .

ostrina, 75 .

petrosa, 112.

saxicola, 75 .

Pyrina, 276.

Parryi, 276.

Pyrula, 113.

modesta, 113.

Radiolites, 107.

gregaria, 61, 107.

Mortoni, 63.

Ranelea, 8, 73.

Californica, 73.

Mathewsonii, 8, 74 .

triquetra, 73.

Rémondia, 270.

furcata, 270 .

Reptescharelia, 107.

IIeermannii, 107.

plana, 108.

Reptescilarellina, 108.

rornuta, 108.

disparilis, 108.

Heermannii, 108.

Reptoporina, 108.

eustomatil, 108.

Rhynchonella, 123, 204, 253.

nitens, 123.

peregrina, 204.

Whitneyi, 204, 253.

RIMELLA, 2:5.

canalifera, 225.

simplex, 225 .

Ringicula, 231.

varia, 231.

Ringinella, 174, 231.

pinguis, 175, 231.

polita, 174, 231.

Rostellaria, 76, 225.

canalifera, 225.

indurata, 76.

simplex, 225.

Sanguinolaria, 94, 238.

Californica, 94, 124.

Whitneyi, 238.

Saxicava, 116.

abrupta, 116 .

Saxidonius, 58, 98.

aratus, 58, 98.

gibbosus, 58, 98.

gracilis, 58, 98 .

Nuttallii, 58, 98.

Scalaria, 78, 223, 261.

bellastriata, 78 .

crebricostata, 78.

Mathewsonii, 223.

subcornata, 78 .

Texana, 261.

Scaphites, 210.

ramosus, 210.

Schizodesma, 20, 91.

abscissa, 20, 91.

Schizopyga, 47, 74.

Californica, 74.

Schizothœrus, 91.

Nuttallii, 91.

Sconsia, 223.

tuberculata, 223. 
Scrobicularia, 92.

viridotincta, 92.

Scurria, 87.

mitra, 87.

Scutella, 37, 109.

Gibbsii, 37, 109 .

interlineata, 110.

striatula, 110.

Semele, 94.

decisa, 94.

pulchra, 94.

Semicassis, 113.

biliratus, 113.

petrosus, 113.

Semitubigera, 109.

tuba, 109.

Septifer, 101, 246.

bifurcatus, 101.

dichotomus, 246.

Serpula, 276.

Sigaretus, 49, 78, 114.

scopulosus, 114.

Siliqua, 89, 233.

Californica, 89.

Oregonensis, 233.

patula, 89.

Siliquaria, 53, 89. edentula, 53,89 .

Srnum, 49, 78, 114.

planicostum, 49, 78 .

scopulosum, 114.

Siphonella, 108.

multipora, 108.

Surlotrochus, 205, 253. curtus, 205, 253.

Solecurtus, 89.

Nuttallii, 89.
Solecurtus subteres, 89.

Solemya, 89.

ventricosa, 89.

Solen, 88, 116, 175, 233.

cuncatus, 175, 233.

curtus, 116.

Diegoensis, 176, 233.

maxima, 89.

patula, 89.

parallelus, 233.

protexta, 89 .

rosaceus, 88 .

Solena, 89, 176, 233.

Diegoensis, 176, 233.

protexta, 89.

Solenoceras, 143.

Sphœnia, 90.

Californica, 90.

SPIROCRYPTA, 228.

pilcum, 228.

Spondylus, 105.

Estrellanus, 105.

Stalagmium, 246.

concentricum, 246.

Standella, 91.

falcata, 92 .

planulata, 92.

Stramonita, 112.

petrosa, 112.

Straparoluus, 224.

lens, 224.

paucivolvus, 224.

Strephona, 75.

Pedroana, 75 .

Struthiolaria, 168.

Surcula, 5, 47, 72, 150, 217.

Carpenteriana, 5, 72. 
Sureula Claytonensis, 217.

inconspicua, 151, 217.

praattenuata, 150, 217.

rarieostata, 217.

sinuata, $150,217$.

Tryoniana, 6, 47, 72.

Sycodes, 160, 221.

cypracoides, 160, 221.

Sycotypus, 111, 113.

modestus, 113.

Ocoyanus, 113.

Oregonensis, 111.

Tamiosoma, 61, 107.

gregaria, 61, 107.

TAPEs, 25, 57, 97, 119, 241, 265.

Conradiana, 241.

cretacea, 241.

diversum, 97.

gracilis, 98.

Hilgardi, 265.

Inezensis, 119.

lintcatum, 97 .

montana, 119.

quadrata, 241.

Staleyi, 57, 98.

staminea, 97 .

tenerrima, 97.

truncata, 25, 98.

Telinna, 92, 117, 182, 237.

xqualis, 182, 238.

albaria, 117.

alta, 92.

arctata, 118.

Ashburnerii, 238.

bitruncata, 117.
Tellina Bodegensis, 92.

calcarca, 124.

Californica, 238.

congesta, 92 .

deeurtata, 237.

Diegoana, 117.

emacerata, 92.

Gouldii, 93.

Hoffmanniana, 182, 237.

Hornii, 238.

inconspicua, 94, 124.

inquinata, 93.

lata, 124.

ligamentina, 93.

longa, 237.

Mathewsonii, 237.

monilifera, 237.

nasuta, 93.

obruta, 117.

Ocoyana, 118.

ooides, 237.

Oregonensis, 93.

parilis, 238.

Pcdroana, 93, 124.

proxima, 124.

quadrata, 237.

Rémondii, 182, 237.

secta, 93 .

sordida, 124.

tenera, 124.

tersa, 93 .

undulifera, 183, 238.

Whitneyi, 238.

Terebra, 162, 223.

Californica, 162, 223.

Terebratelia, 35, 204, 253.

obesa, 253 . 
Terebratella Whitneyi, 35, 204, 253.|Trigonia Evansana, 247, 269.

Terebratula, 123. nitens, 123.

Teredo, 115. substriata, 115.

TESSAROLAX, 226. distorta, 226 .

Thalotia, 84 . caffea, 84 .

Theliostyla, 170, 229. triangulata, 170, 229.

Thetis, 186, 241. elongata, 186, 241.

Thracia, 90, 117, 235. mactropsis, 117. occidentalis, 235. subtruncata, 235 . trapezoides, 90.

Tornatella, 115. elliptica, 115.

Tornatina, 88. culcitella, 88.

Trachytriton, 154, 218. Diegoensis, 218. fusiforme, 219. Tejonensis, 218.

Trapezium, 241. carinatum, 241.

Tresus, 91. maximus, 91.

Trigona, 96. crassatelloides, 96. tantilla, 96.

Trigonia, 196, 247, 269. æquicostata, 196, 248. crenulata, 269.

Emoryi, 269. PAL. VOL. II.-39
Evansii, 247.

Gibboniana, 247.

Hondaana, 247.

Mooreana, 269.

Tripter $, 1,69$. clavata, 1, 69 .

Triton, 73.

Oregonensis, 73.

Tritonidea, 148.

Tritonium, 73, 154, 218.

Californicum, 154, 218.

Diegoensis, 218.

fusiforme, 155, 219.

Hornii, 218.

luridum, 70.

Oregonensis, 73.

paucivaricatum, 218.

Tejonensis, 154, 218.

Whitneyi, 218.

Tritonofusus, 146, 147, 216.

cretacea, 146, 216.

Trochiscus, 85.

convexus, 85 .

Norrisii, 85.

Trochita, 15, 51, 81, 115.

costellata, 81.

Diegoana, 115.

filosa, 15,81 .

inornata, 51, 81 .

Trochosmilia, 253.

granulifera, 254.

striata, 253.

Trochus, 83.

Adamsianus, 84 .

annulatus, 83.

aureotinctus, 84 . 
'Trochus balanarum, 83.

brunneus, 84.

canaliculatus, 83.

costatus, 83.

gibberosus, 83.

inequalis, 83.

marcidus, 84 .

ochraceus, 83.

Pfeifferii, 84.

undosus, 83.

Trophon, 2, 14, 70.

multicostatus, 70 .

Orpbens, 70.

ponderosus, 2,70 .

squamulifer, 44, 70, 123.

tenuisculptus, 70 .

Truncaria, 74 .

corrugata, 74 .

'Turbinella, 157, 220.

crassitesta, 157, 220.

Turbinolia, 276.

Texana, 276.

Turbonilla, 12, 79, 114.

aspera, 12, 79.

papillosa, 114.

Turcica, 16, 84 .

Turnus, 233.

plenus, 233 .

Turrilites, 138, 213.

Oregonensis, 138, 213.

Turris, 5, 112, 217.

Claytonensis, 217.

raricostata, 217 .

transmontana, 112.

varicostata, 217.

Turritella, 14, 80, 114, 169, 227, 263.
Turritella Chicoensis, 228.

Cooperii, 80 .

dispassa, 228.

Hoffmannii, 14, 80.

Inezana, 81 .

infragranulata, 227.

infralineata, 227.

irrorata, 227.

Jewettii, 80.

Martinezensis, 169, 228

Ocoyana, 114.

planilateralis, 227.

robusta, 228.

Saffordii, 228.

seriatim-granulata, 227, 263.

Uvasana, 228.

variata, 80 .

Veatchii, 228.

Tylostoma, 261.

mutabilis, 261.

Typhis, 214.

antiquus, 214.

Unio, 190, 245.

Hubbardii, 190, 245.

penultimus, 245 .

UrosycA, 159, 221.

caudata, 159, 221.

VANIKORO, 115.

Diegoana, 115.

Varigera, 261.

Venericardia, 101.

borealis var. ventricosa, 101

Venus, 22, 55, 98, 118, 184, 238.

xquilateralis, 184, 239. 
Venus angustifrons, 118.

bisecta, 28, 99.

brevilineata, 95 .

Californica, 94.

callosa, 96.

Kennerlyi, 22, 55, 94, 95.

lamellifera, 95, 97.

lenticularis, 239.

maxima, 98.

Nuttallii, 95.

Pajaroensis, 118.

pertenuis, 22, 55, 95.

rhysomia, 96.

rigida, 97.

securis, 95.

succincta, 94 .

tantilla, 96.

tetrahedra, 239.

varians, 239.

Veatchii, 238.

Vola, 104.

dentata, 104.

Volutilithes, 76, 220 .

Californica, 225.

indurata, 76.

Navarroensis, 220.

Yoldia, 31, 58, 103.

Cooperii, 31, 59, 103.

impressa, 58, 103.

nasuta, 58, 103.

Zirph eA, 18, 52, 88.

crispata, 88.

dentata, 18, 124.

Gabbii, 52, 88.

Zizyphinus, 17, 83, 170, 229.

annulatus, 83.

canaliculatus, 83 . 



\section{I 14.11}

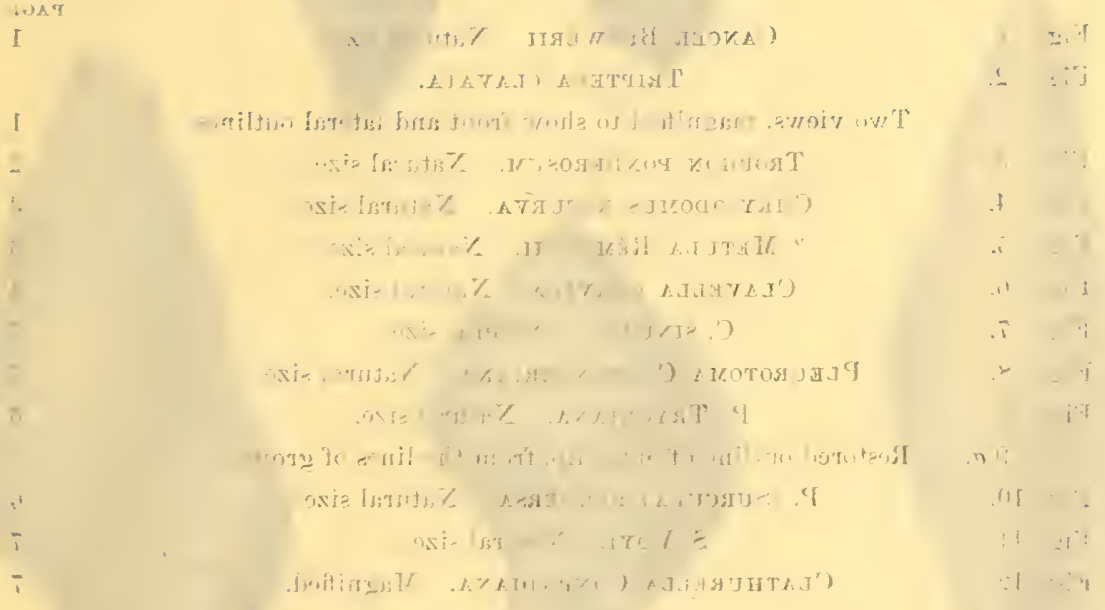




\section{P L A T E I.}

Fig. 1.

Fig. 2.

Fig. 3.

Fig. 4.

Fig. 5.

Fig. 6.

Fig. 7 .

Fig. 8.

Fig. 9.

$9 a$

Fig. 10.

Fig. 11.

Fig. 12.
Cancer Brewerit. Natural size.

PAGE

Triptera clavata.

Two views, magnified to show front and lateral outlines.

Trophon ponderosum. Natural size.

Chrysodomus recurva. Natural size.

? Metula Rémondi. Natural size.

Clavella gravida. Natural size.

C. sinuata. Natural size.

Pleurotoma Carpenteriana. Natural size.

P. Tryoniana. Natural size.

Restored outline of outer lip, from the lines of growth.

P. (Surcula) Perversa. Natural size.

S. VoYi. Natural size.

Clathurella Conradina. Magnified.
1

3

3

4

5

5

6

6

7

7 


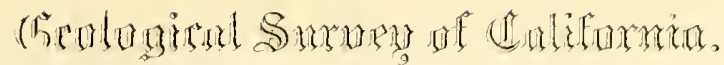

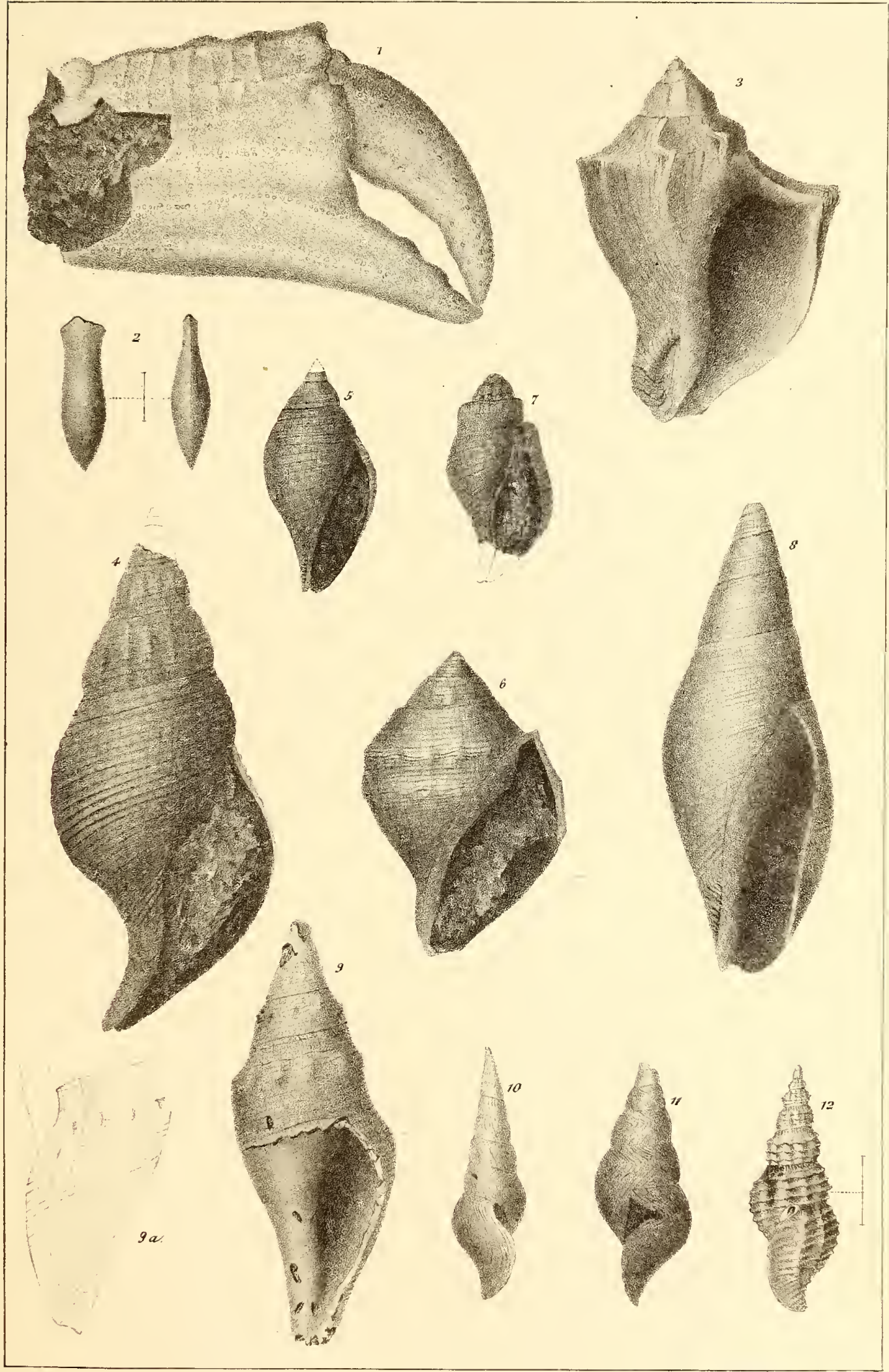



$\begin{array}{llll}1106 & 11\end{array}$

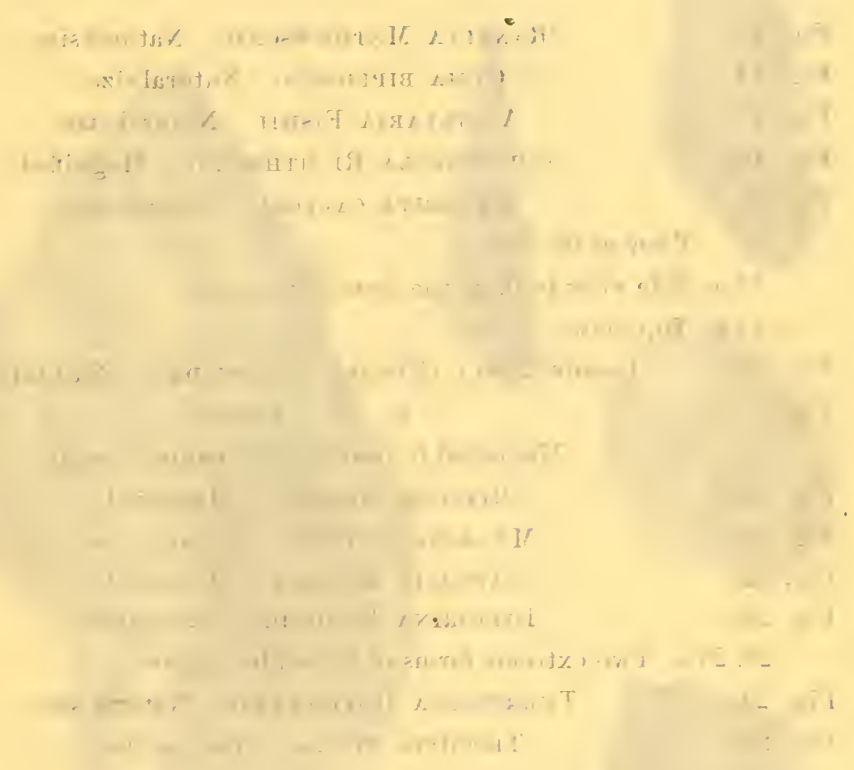




\section{P L A T E I I.}

Fig. 13.

Fig. 14.

Fig. 15.

Fig. 16.

Fig. 17.
Ranella Mathewsonir. Natural size.

Cuna biplicata. Natural size. Ancillaria Fishir. Natural size.

Columbella Richthofeni. Magnified.

Nevertta Callosa. Natural size.
PAGE

8

9

9

10

10

View of the base.

17 a. Side view to show the form of the callus.

17 b. Top view.

Fig. 18.

Cancellaria (Euclia) tritonidea. Natural size.

Fig. 19.

C. (E) Vetusta.

12

Magnified to nearly twice natural length.

Fig. 20. Bittuim asperum. Magnified.

Malania Ta rlori. Natural size.

13

Fig. 21.

Fig. 22.

Fig. 23.

Lithasia antiqua. Magnified.

Littorina Rémondi. Magnified.

13

14

23,23 a. Two extreme forms of a variable species.

Fig. 24.

Turritella Hoffmanni Natural size.

Fig. 25.

Trochita filosa. Natural size.

25 a. Side view. 


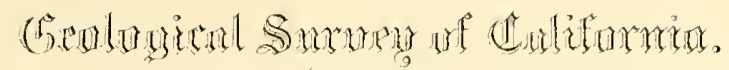
PATIEDETOLDLY. VDL。II.

(Tertiary)

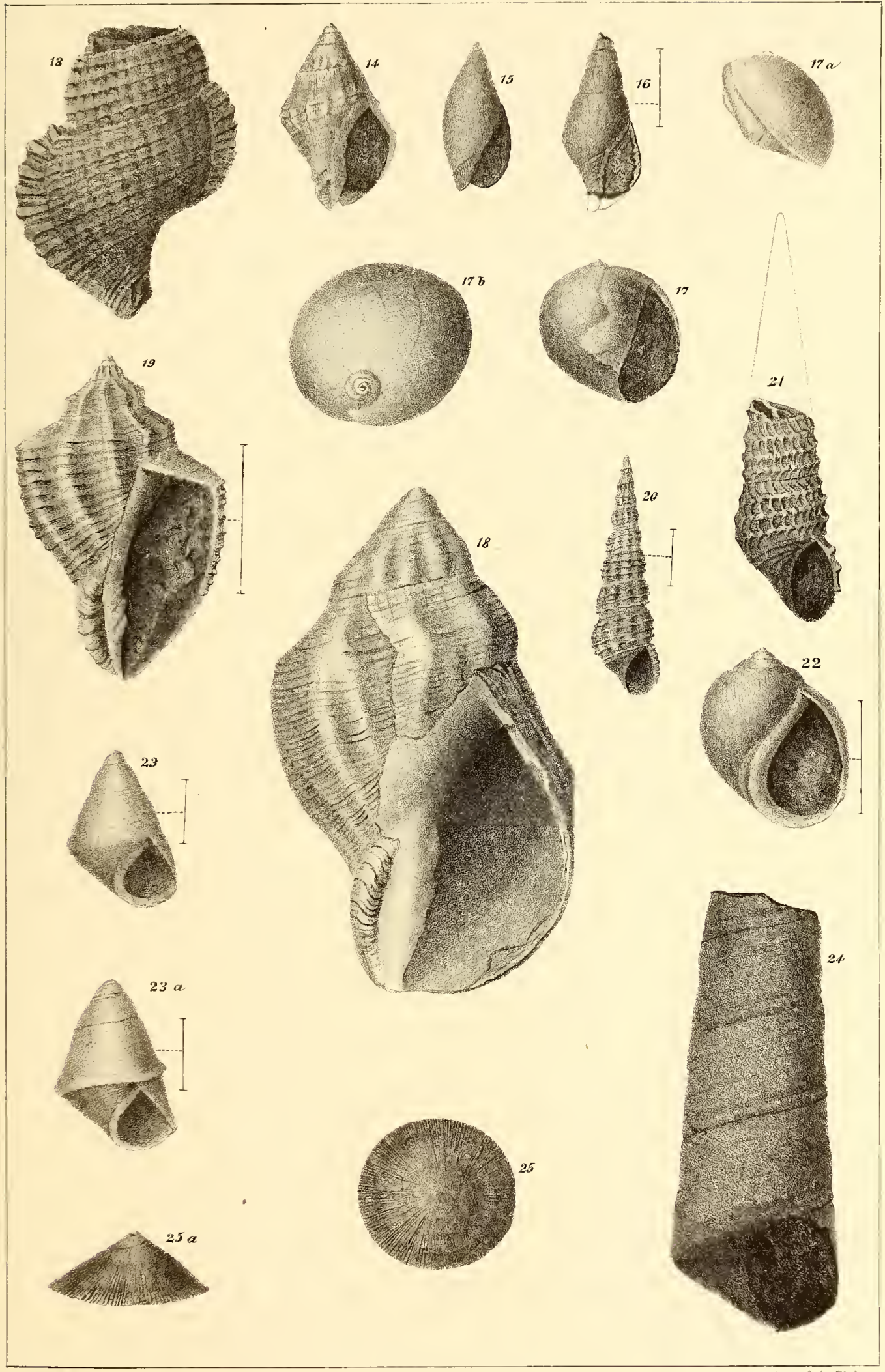

ristis a di

Tas Sunciair \& Son Inthe Phala 

s.

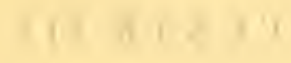

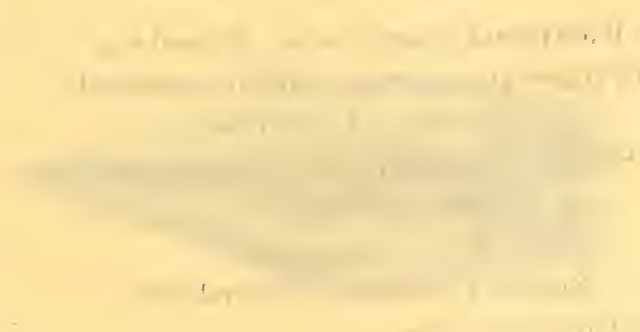

$\checkmark$ 


\section{P L A T E I II.}

Fig. 26.

Fig. 27.

Fig. 28.

Fig. 29.

Fig. 30.

Fig. 31.
Pachypoma biangulata. Natural size.

Ptychostylis caffea. Slightly magnified.

Calliostoma tricolor.

Slightly magnified from a recent specimon.

Indet. Natural size.

Indet. Natural size.

ZIRPHA DENTATA. Natural size.
PAGE

31 a. Top view of the dorsal plate. 


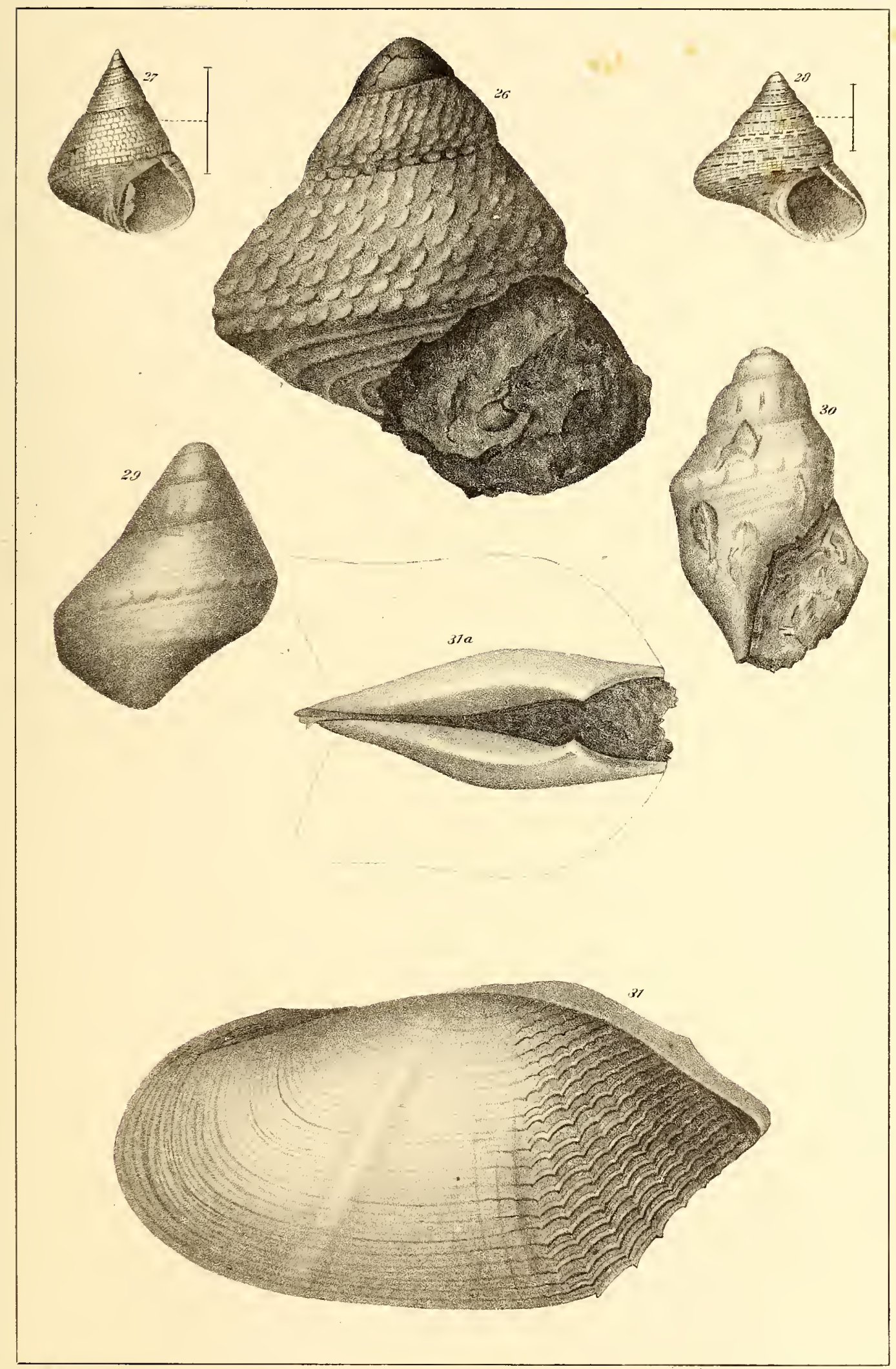

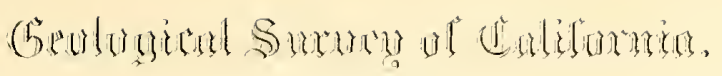

PATEEDADEDFY, YOH, LH,

('lertiary )

PTANTI:

T. Sinciarr lith. Philadeipha. 




\section{P L A T E I V.}

Fig. 32.

Fig. 33.

Fig. 34 .
Pandora scapha. Magnified. Hemimactra lenticularis. Natural size. Schizodesma abscissa. Natural size.
PAGE

18

19

20

$34 a$. View of the posterior end. 


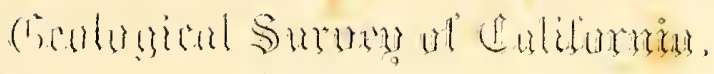







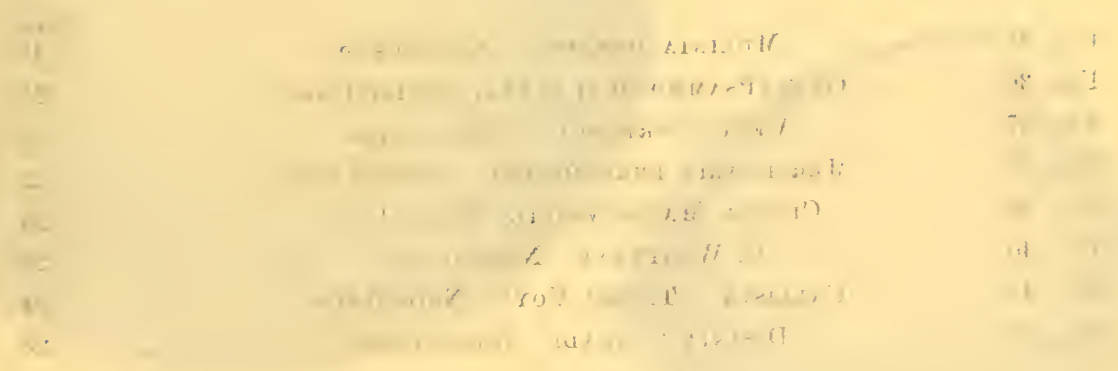




\section{PLATE V.}

Fig. 35.

Fig. 36.

Fig. 37.

Fig. 38.

Fig. 39.

Fig. 40.

Fig. 41.

Fig. 43.
Mulinia densata. Natural size. Gari (Psammocola) alata. Natural size. Venus pertenuts. Natural size. Mercenaria perlaminosa. Natural size. Chione Mathewsonit. Natural size. C. Whitneyi, Natural size. Callista (? Tapes) Voyt. Natural size. Dosinia Conradi. Natural size.
PAGE 


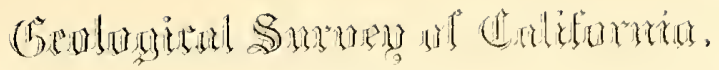

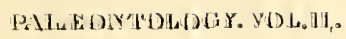

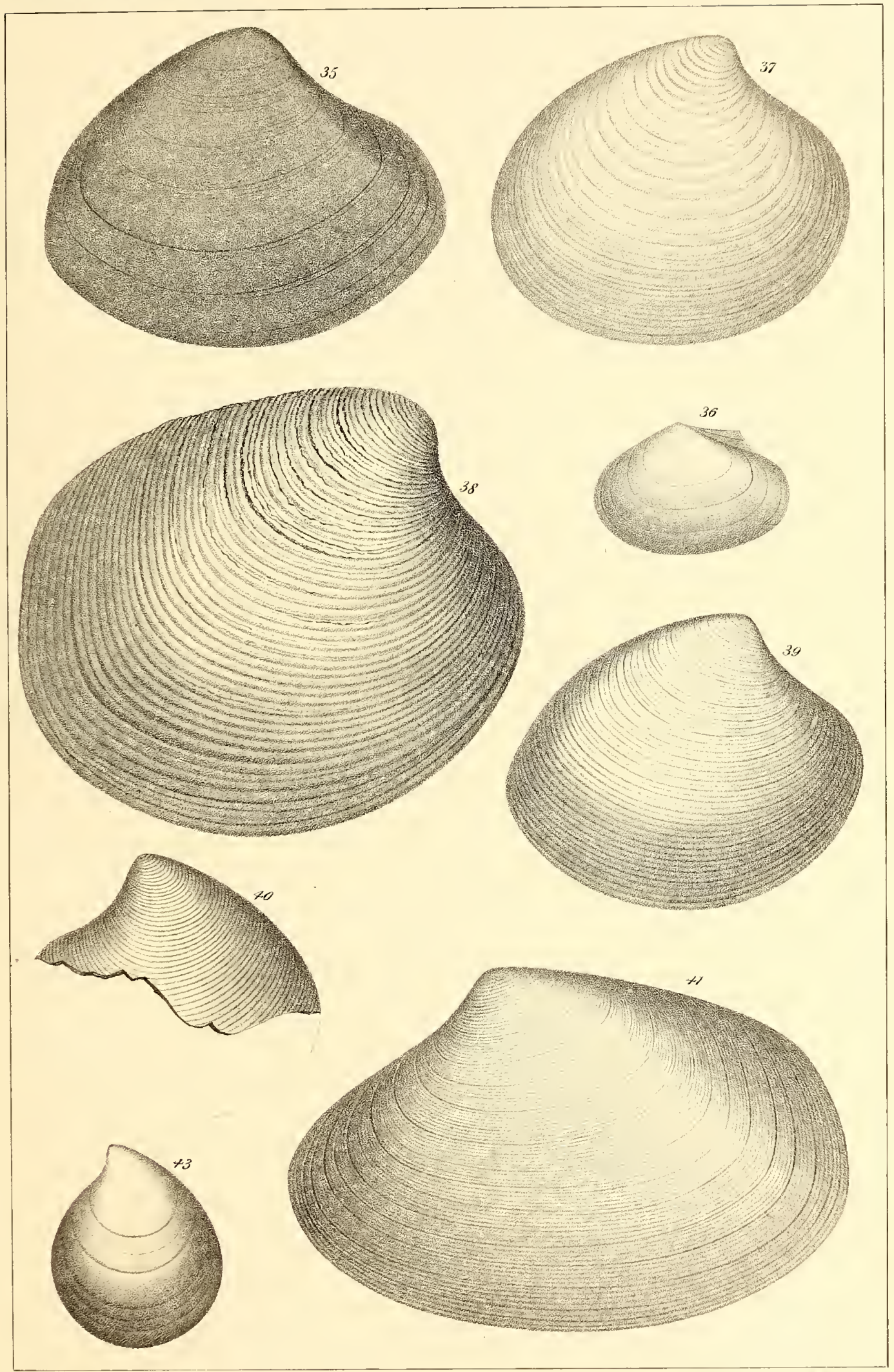




PLA T E V I.

Fig. 47.

Pseudocardium Gabbir. Natural size.

PAGE

47 a. End view.

47 b. c. Hinge of the two valves.

$47 d$. Side view to show the prominence of the enormous lateral teeth. 


$$
0^{a}
$$





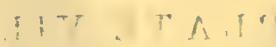
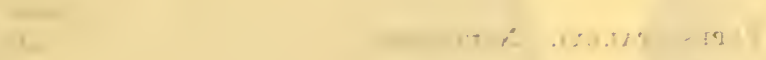

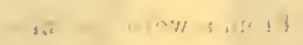

\begin{tabular}{|c|c|c|c|}
\hline- & $\rightarrow 1=1011+26$. & 1 & 4 \\
\hline 7 & $1.1 .1 .7 \%$ & $\prime$; IA & : \\
\hline 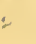 &.$\quad \%$ & 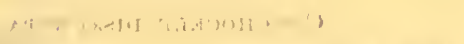 & 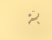 \\
\hline
\end{tabular}

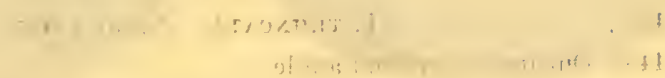




\section{P L A T E VII.}

Fig. 42.

Fig. 44.

44 a. Outline of cardinal angle.

Fig. 45.

Fig. 46.

Fig. 48.
Tapes Staleyi. Natural size.

$$
\text { From a worn specimen. }
$$

?T. TRUnCAta. Natural size.

Cyrena Californica. Natural size.

Cardium Meerianum. Natural size.

Conchocele Disjuncta. Natural size.
PAGE

24

25

26

27

28

$48 a$. View from above.

$48 \mathrm{~b}$. Hinge. 


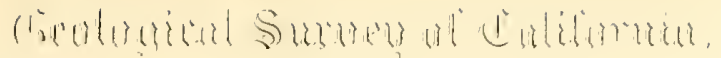

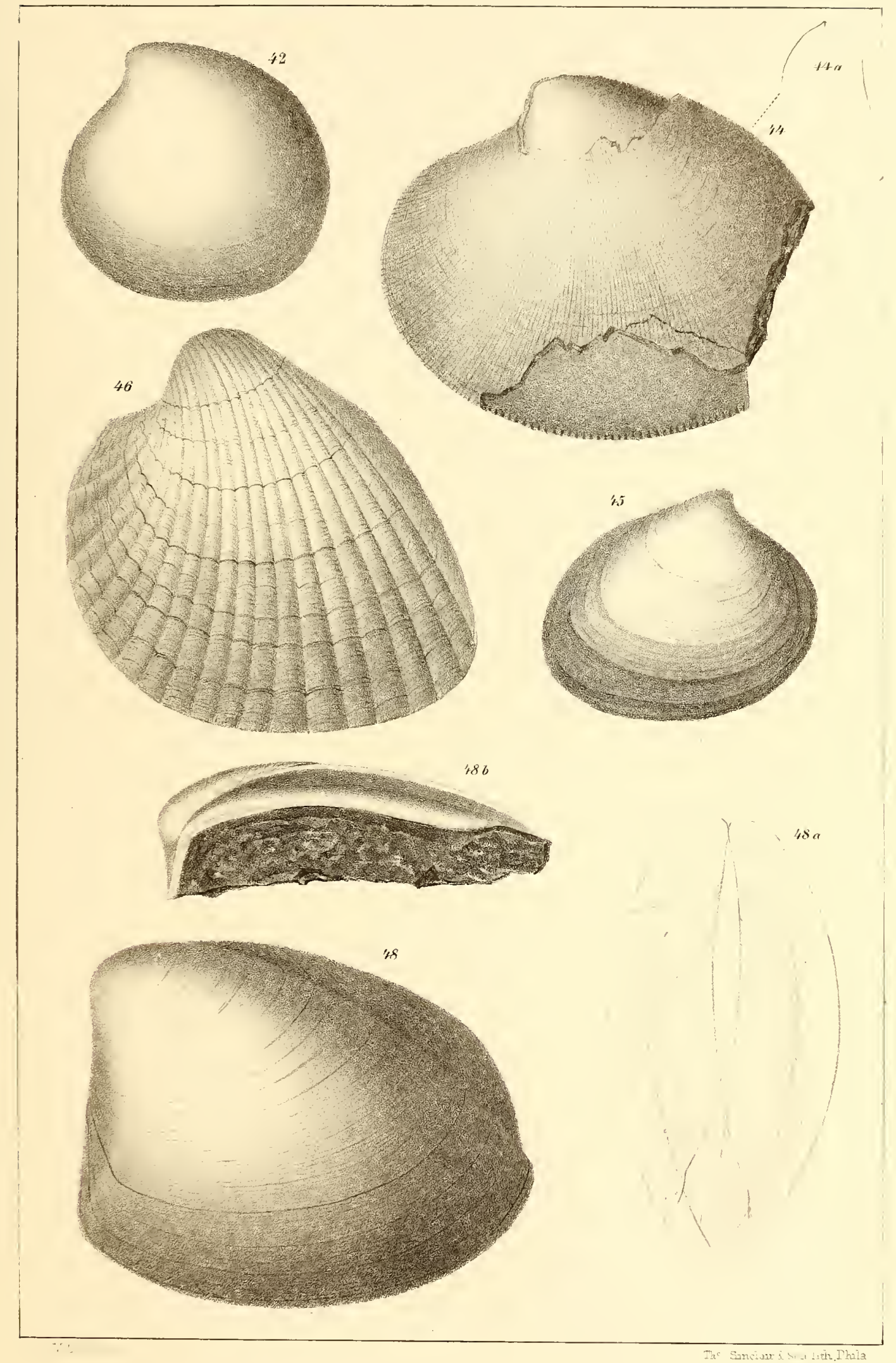






\section{PLA T E VIII.}

Fig. 49. Lucina (Here) Richthofeni. Magnified.

PAGE

$49 a$. Side view showing the convexity and the position of the lunular pit. $49 \mathrm{~b}$. Hinge.

Fig. 50 .

Crassatella collina. Natural size.

29

Fig. 51 .

Mrtilus Mathewsonir. Natural size.

30

Fig. 52.

Modiola multikadiata. Natural size.

30 


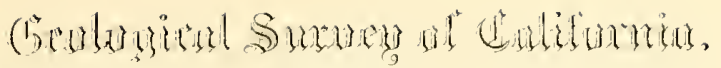

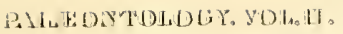

('lerijary)

PTANTHE HS

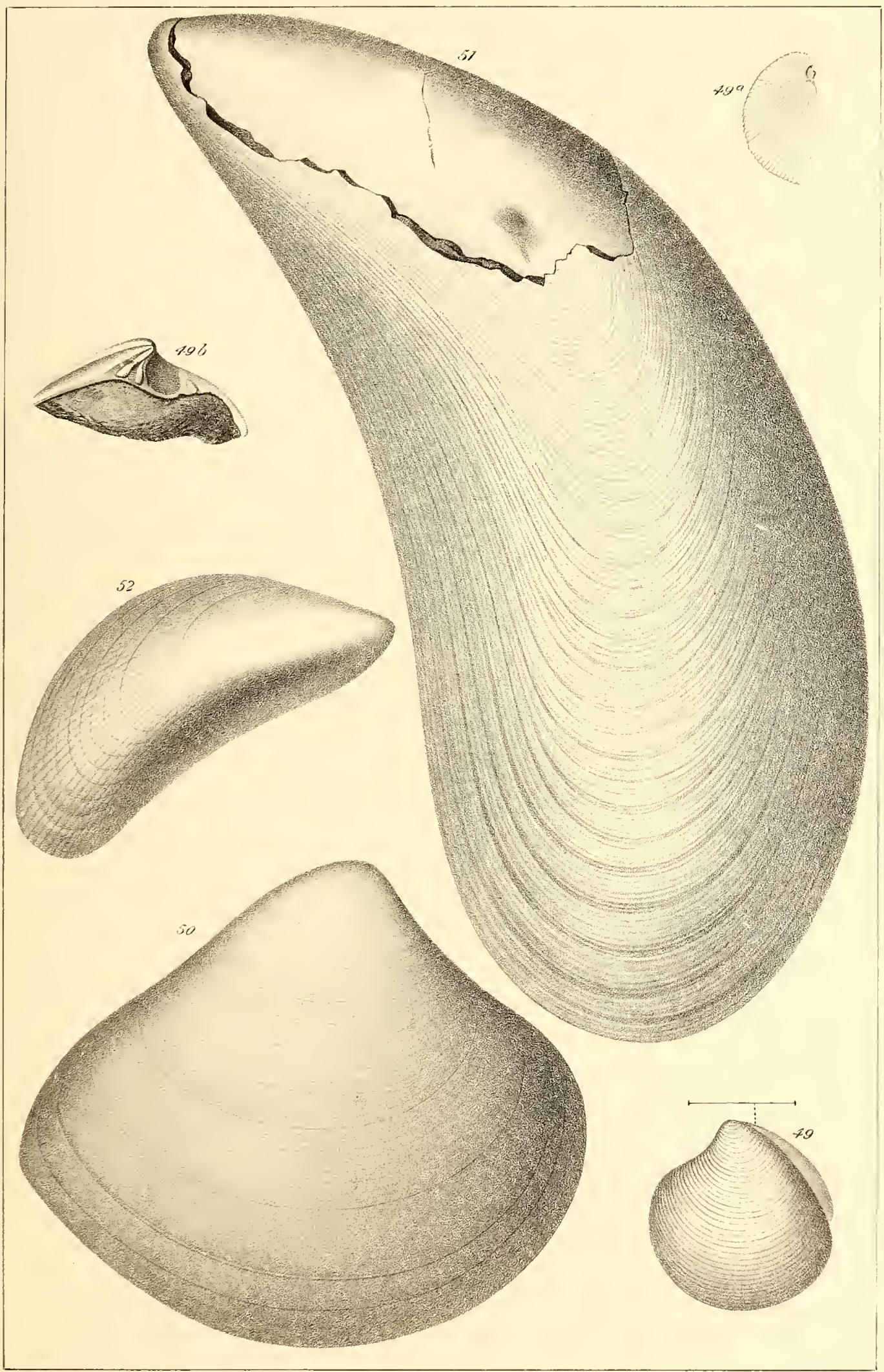

Wir Gate del

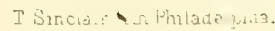





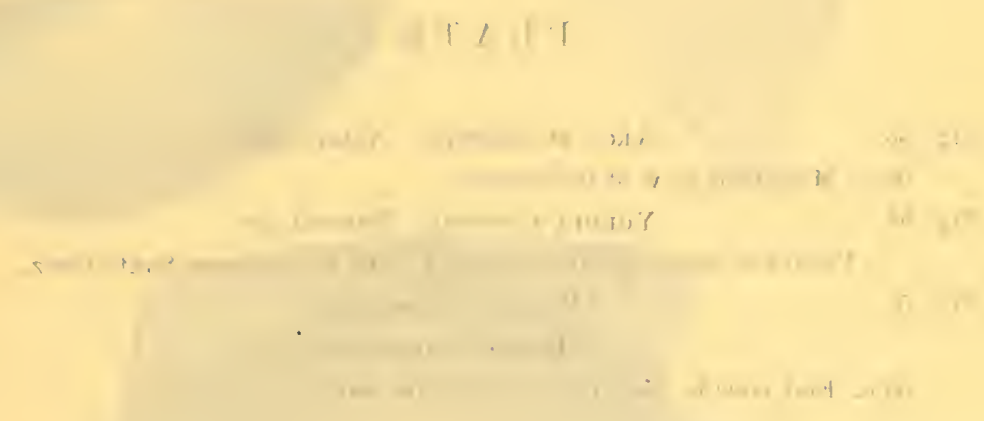




\section{P L A T E IX.}

Fig. 53. Arca sulcicosta. Natural size.

PAGE

53 a. Magnified view of the surface.

Fig. 54 .

Yoldia Cooperi. Natural size.

From the recent specimen found by Dr. Cooper near Santa Cruz.

Fig. 55 .

Pecten Cerrosensis.

One-half natural size.

$55 a$. End view to show convexity of the valves. 


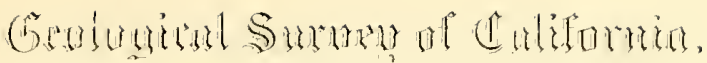

PIL EDYTDLDGY. TOH. IT

('Terting)

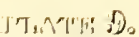
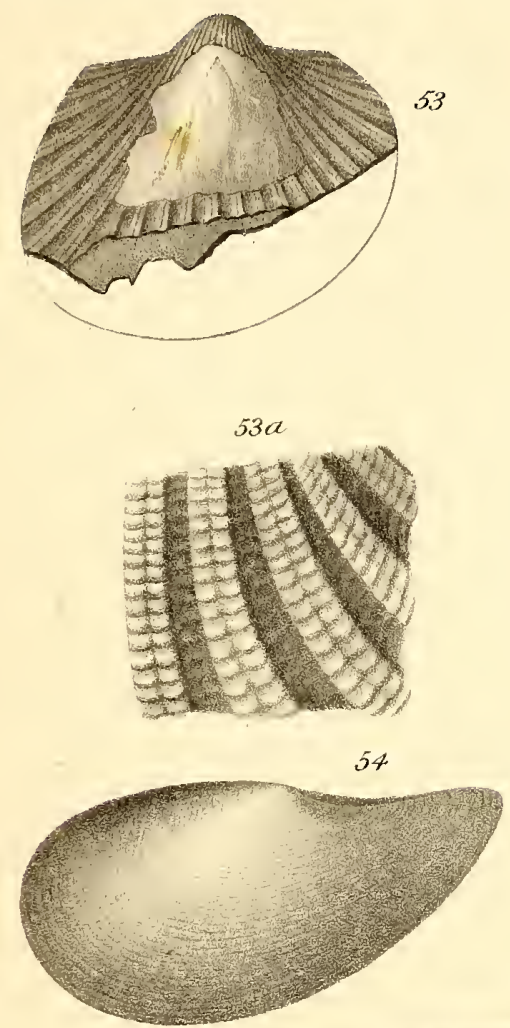
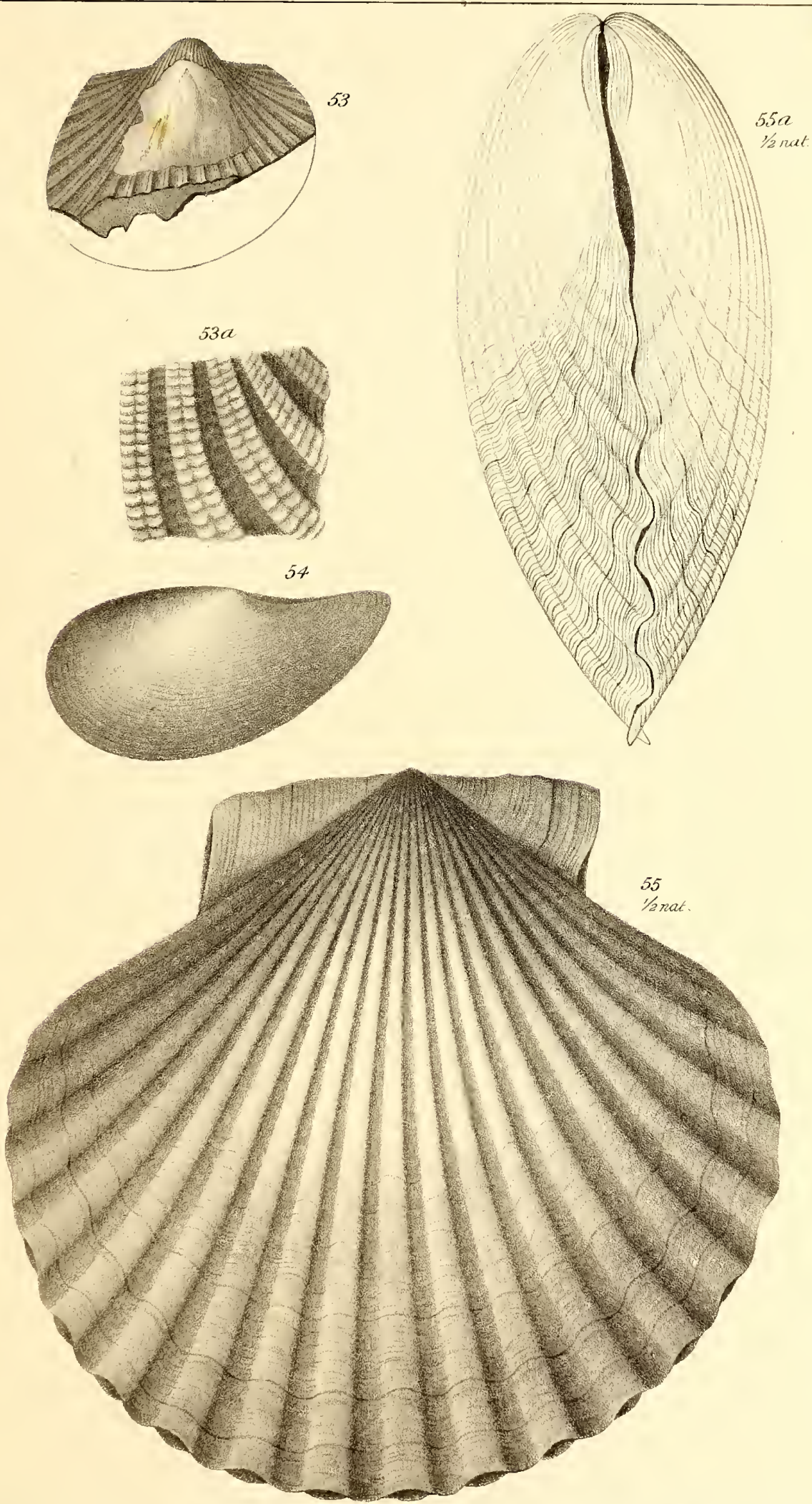



$$
+1+, 1
$$

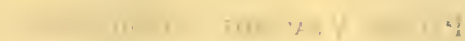
$\therefore$

$$
\begin{aligned}
& \text { n }, \cdots,+,
\end{aligned}
$$

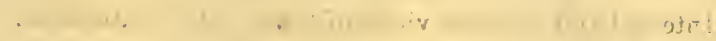




\section{P L A T E X.}

Fig. 56.

Fig. 58, $58 \alpha$.
Pecten Veatchis. Natural size.

Ostrea AtTwoodit.
PAGE

32

33

Internal and external views of lower valve, natural size. 


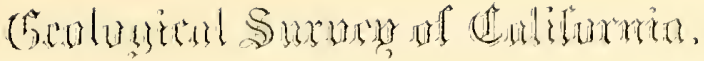

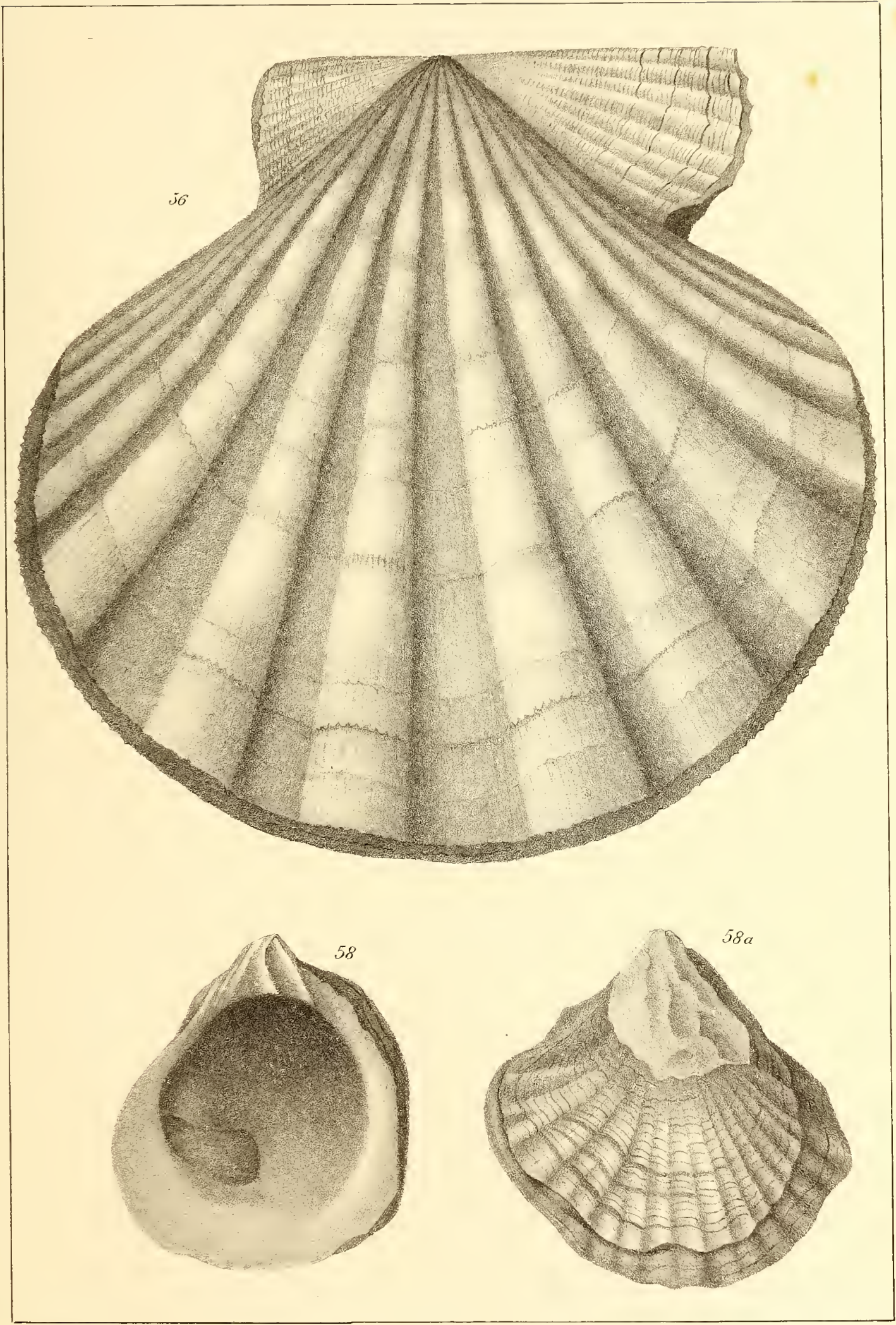

Uat ael

-... S-mciair \& Ser intiv Phila 



$$
1+4141
$$

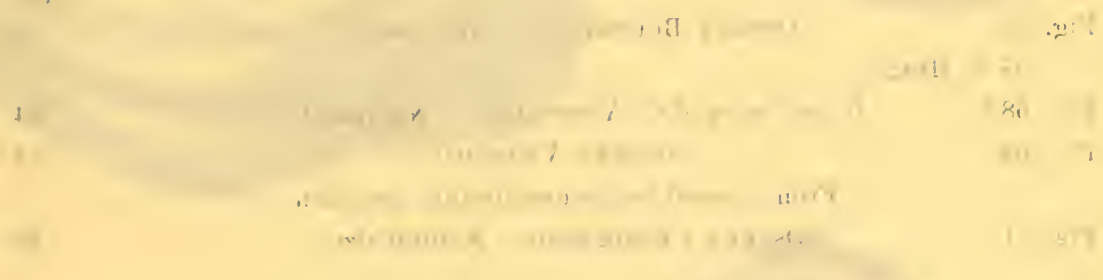




\section{P L A T E X I.}

Fig. 57.

$57 a$. Hinge.

Fix $58 b$.

Upper valve of O. Aтtwoodir? Natural size.

34

Fig. 59 .

Ostrea Veatchit.

34

From a small and unusually thin specimen.

Fig. 61.

Ostrea Cerrosensis. Natural size. 


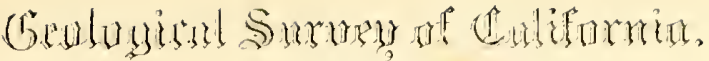

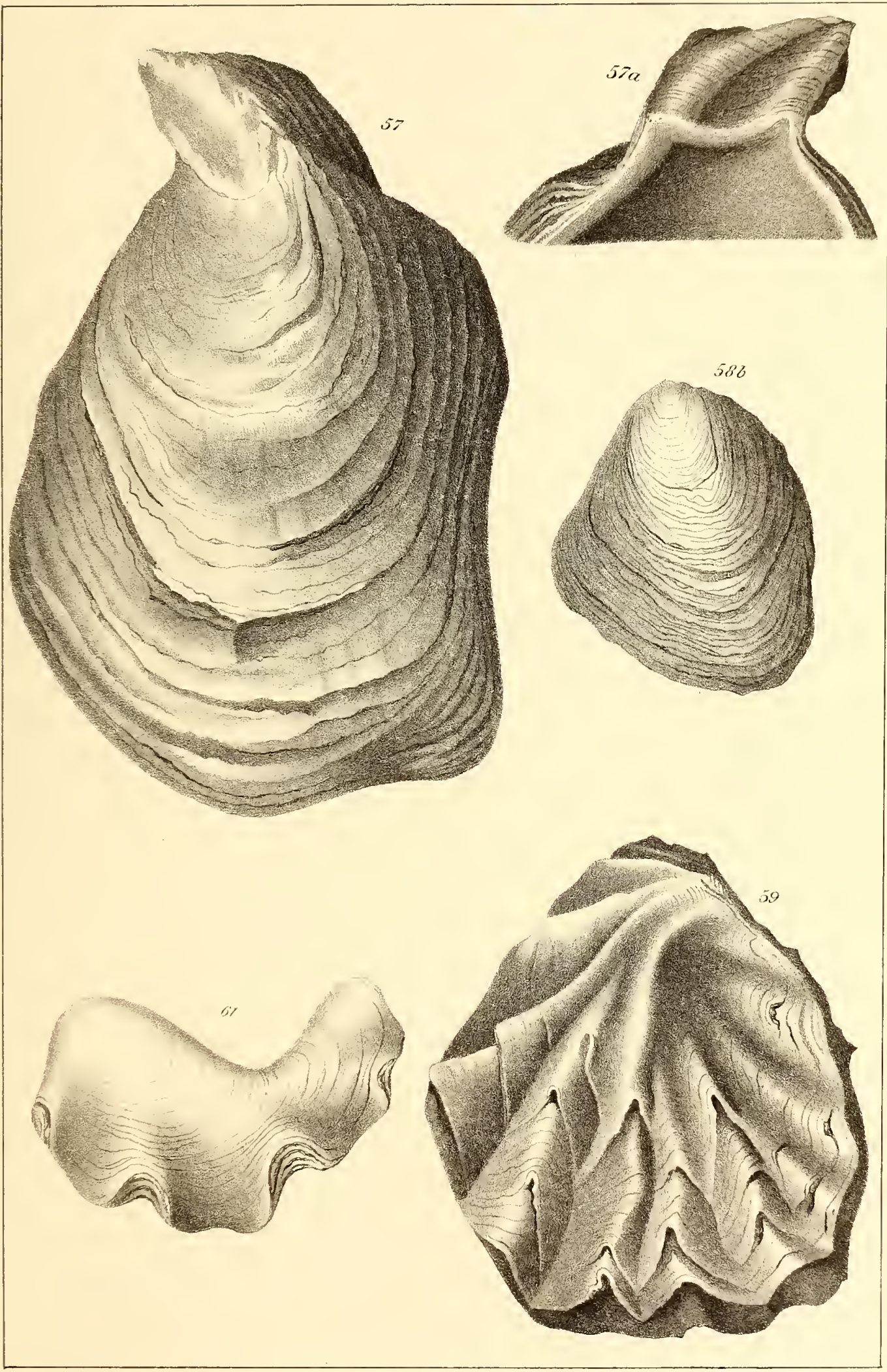




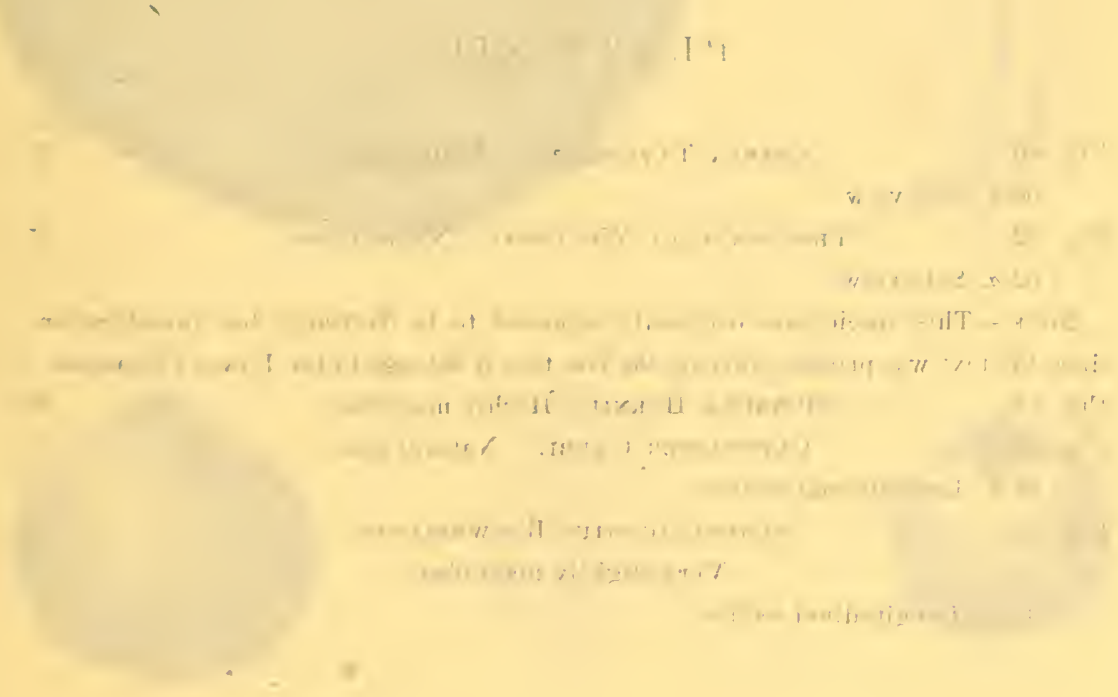

. 


\section{PI A T E XII.}

Fig. 60 .

Ostrea Tayloriana. Natural size.

fio $a$. Side view.

Fig. 62. Terhebatella Whitemi. Natural size.

6. a. Side view.

Noтk.-This species was originally supposed to be Tertiary; but investigations since the text was printed, develop the fact that it belongs to the Lower Cretaceous.

Fig 63. Morrisia Hornir. Highly magnified.

36

Fig. 64. C'lypenster Gabbir. Natural size.

36

64 a. Longitudinal section.

Fig. 65 .

fichinahachnius Brewerianus.

36

Very slightly magnified.

(35) a. Longitudinal section. 


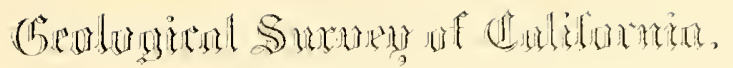
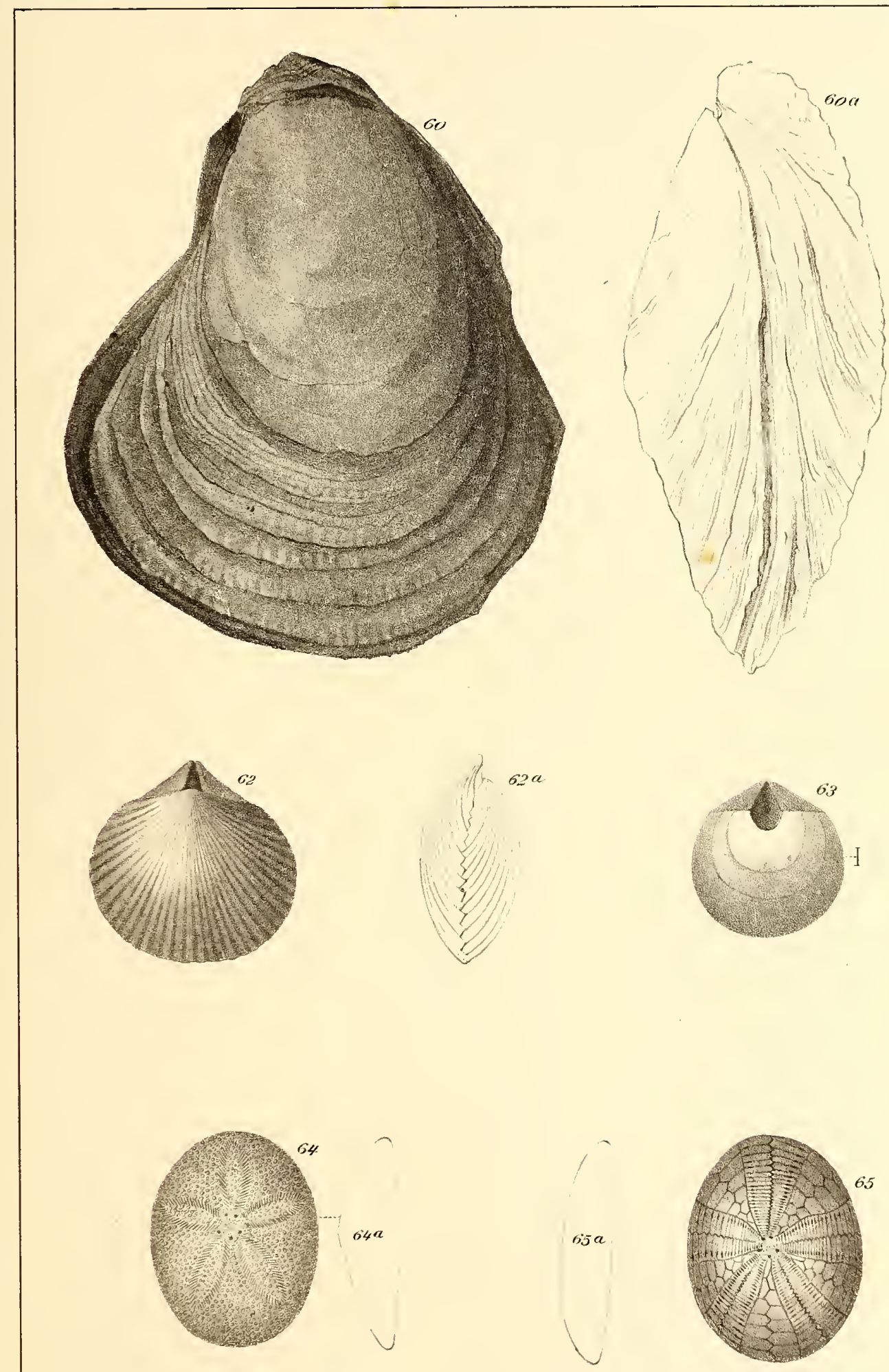




\section{P L A T E X I I I.}

Fig. 66.

66 a. Section

Fig. 67 67 a Elevation.

Fig. 68. $68 a$. Side outline.
Scutella Gibbsit. Natural size.

Astrodapsis Whitneyi. Natural size.

Astrodapsis tumidus. Natural size. Asterias Remondit. Natural size. View of one of the arms. 37 
(b)
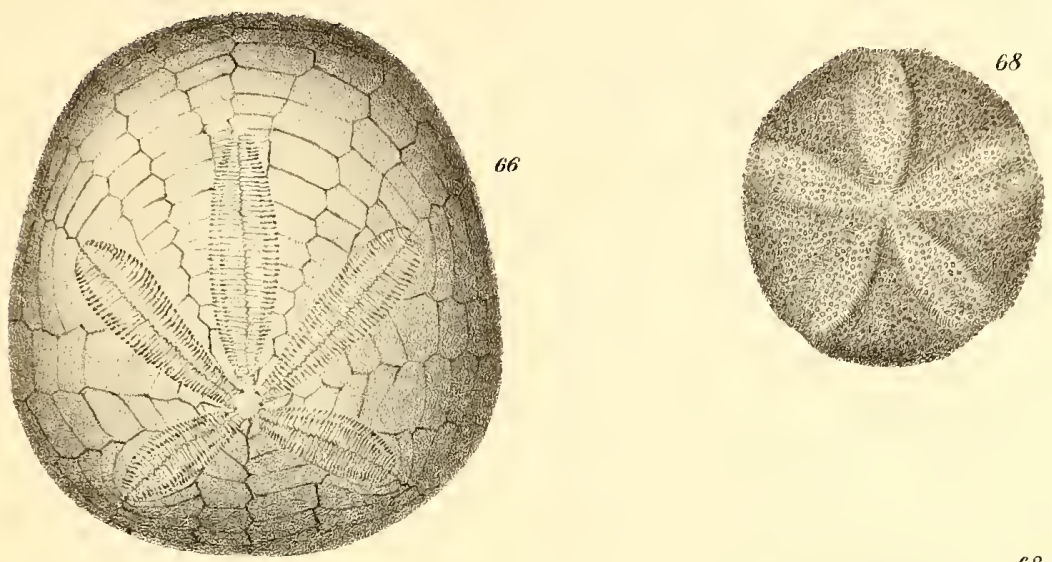

$68 a$
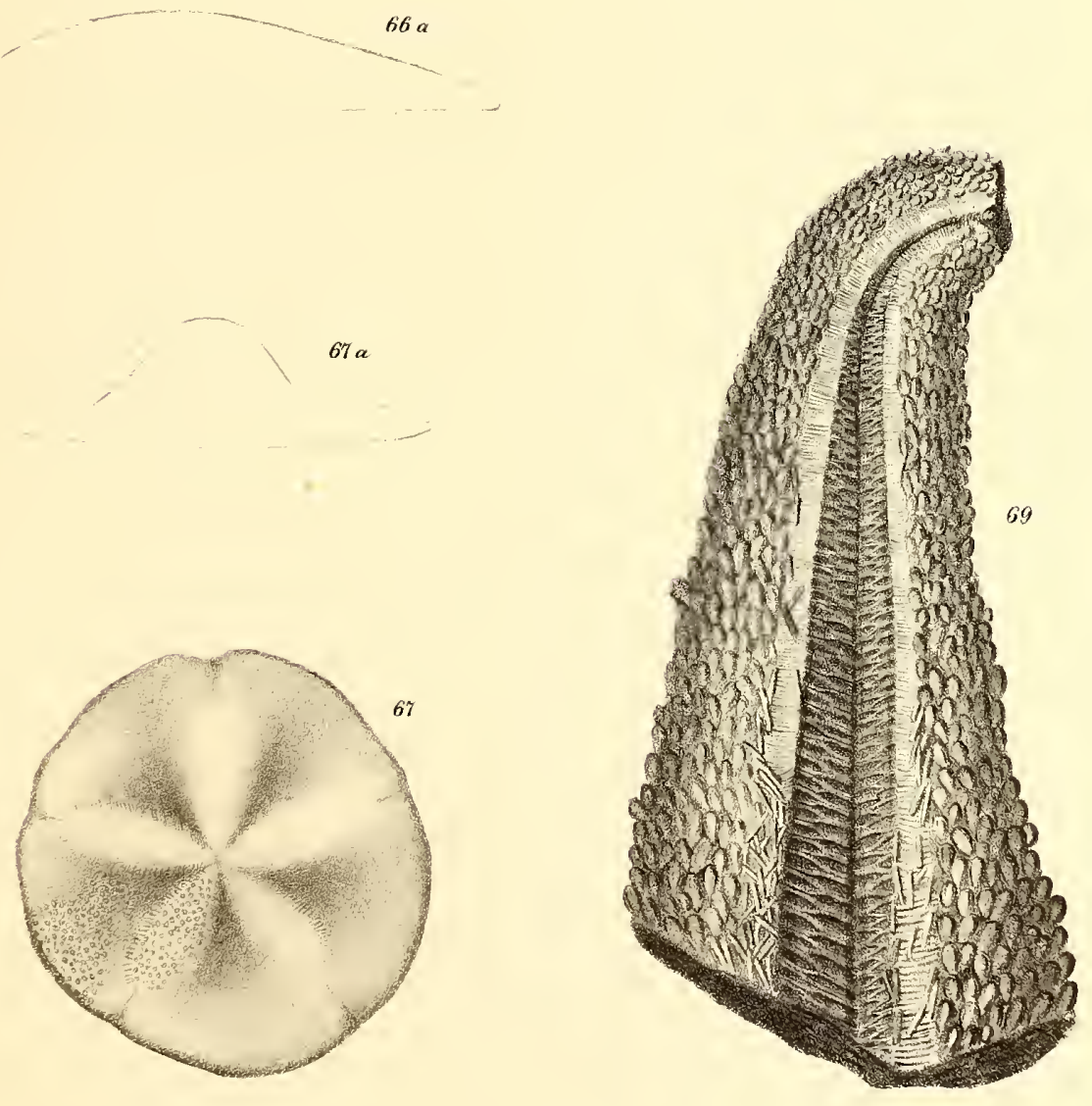

N" LI Gabt del 




\section{PLATE XIV.}

Fig. 1.

Fig. 2.

Fig. 3.

Fig. 5.

Fig. 6.

Fig. 7.

Fig. 8.

$8 \alpha$. Side view.

Fig. 9.

9 a. Side view.
Muricidea paucivaricata.

Neptunea altispira.

N. HUMERosa.

45

The apex is restored from smaller speeimens.

Ficus PyRIFORMIS. $\quad 48$

F. Nodiferus. 48

SiNuM PLANicostum. $\quad 49$

Cancellaria altispira. $\quad 50$

Trochita INORNATA.

ACMraA RUDIS.

51 


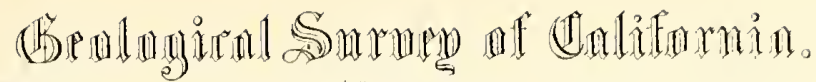

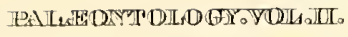
('Tertiary)

PInATE 74.

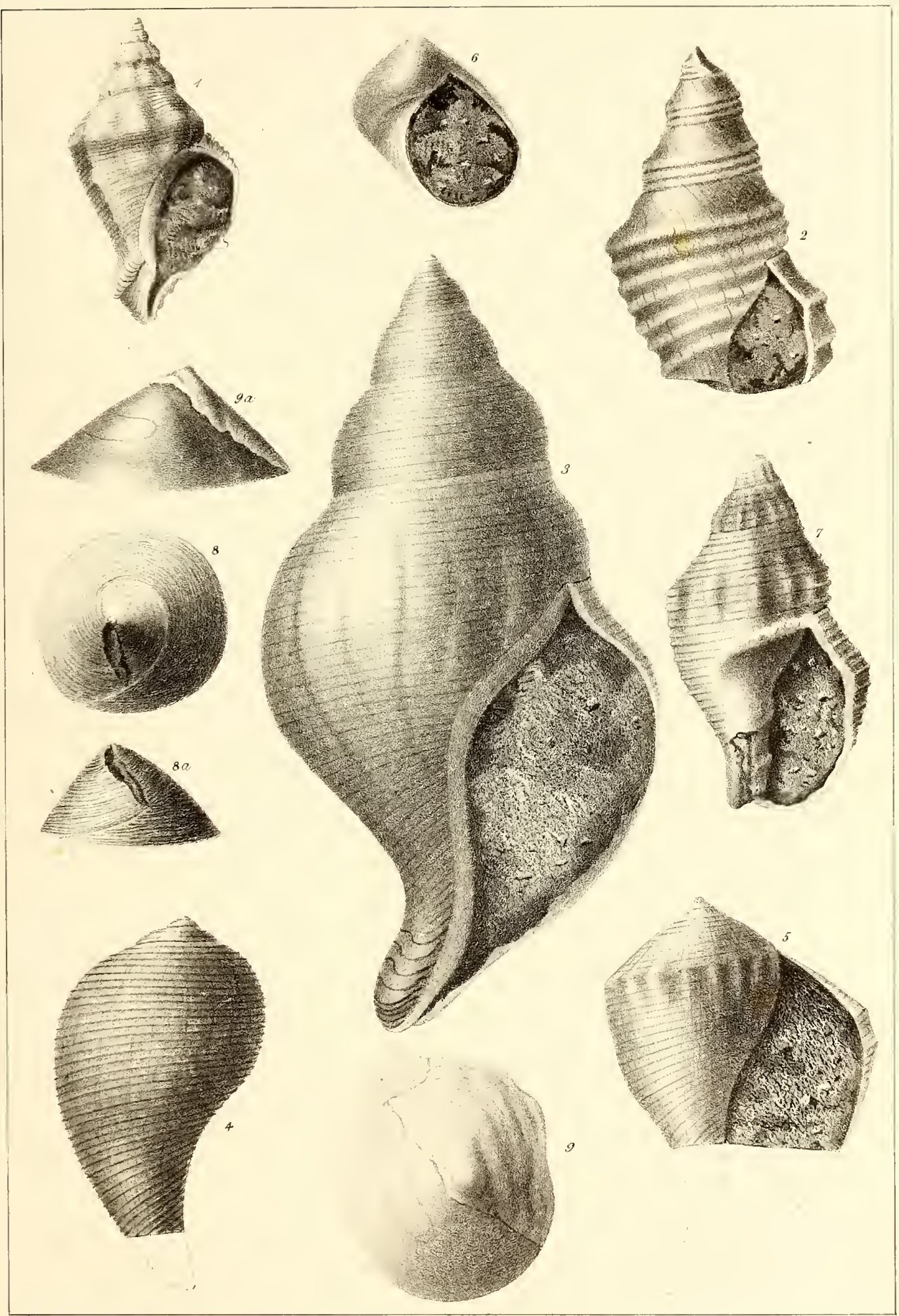

W. M. Gabb, del

Bowen\& Co Iith. Philada 




\section{PLATE XV.}

Fig. 10.

Fig. 11.

Fig. 12.

Fig. 13, $13 a$.

Fig. 14.

Fig. $15,15 \alpha$.

Fig. 16.
Zirphea Gabbil.

? Siliquaria edentula.

Clidophora punctata.

? Hemimactra occidentalis.

Mericenaria perlaminosa.

Caryatis Barbarensis.

Dosinia Mathewsoonif.
PAGE

52

53

54

54

55

56

57 


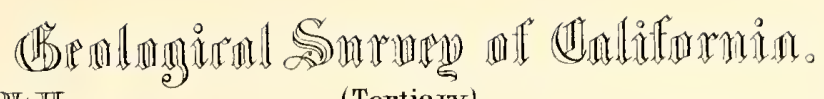

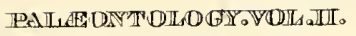

(Tertiary)

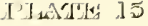

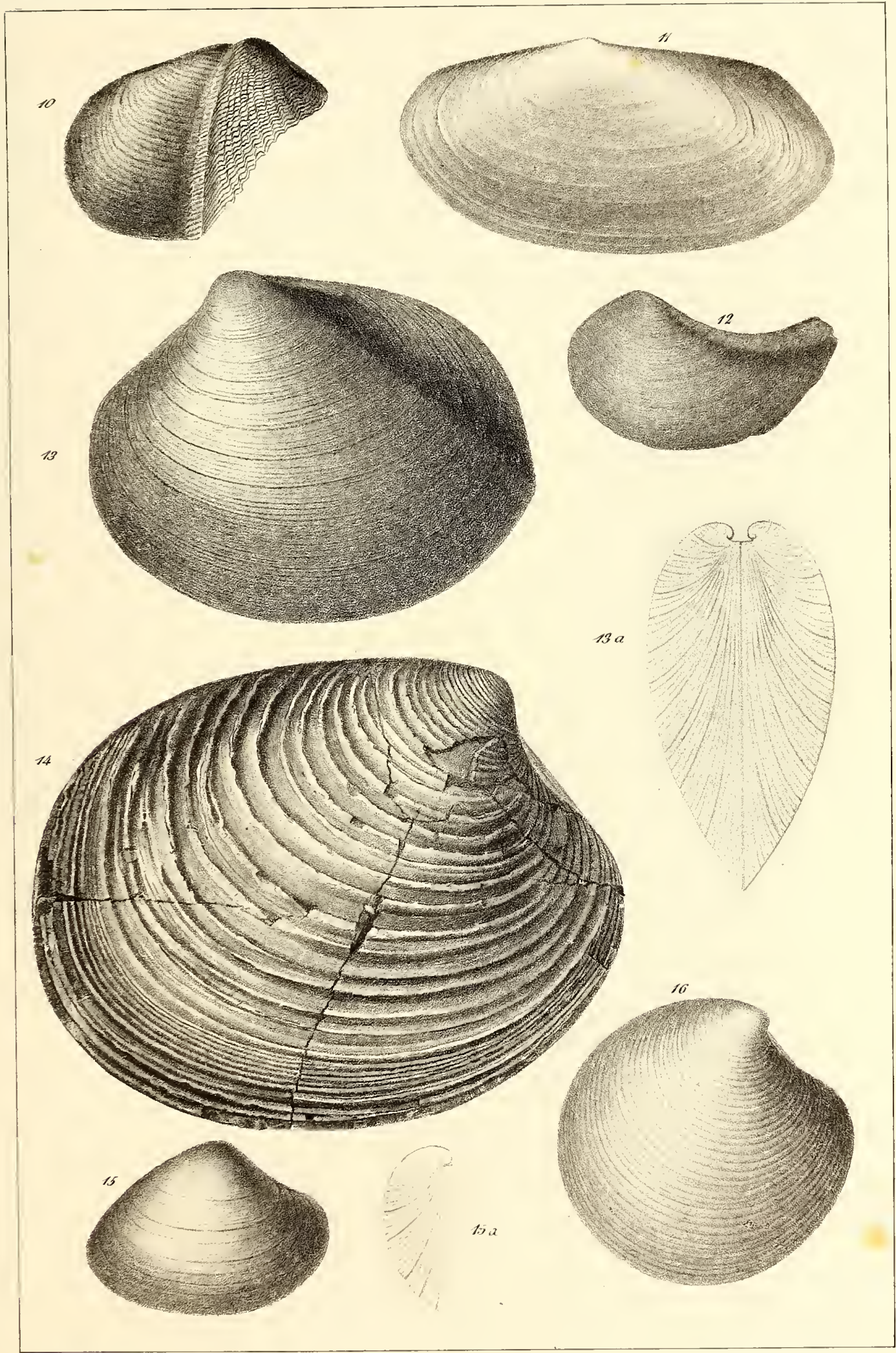

W. M Gabb, del 




\section{PLATE X VI.}

Fig. 17.

17 a. Hinge.

Fig. 18.

$18 \alpha$. End view.

$18 b$. Hinge.

Fig. 19.

Pecten Peckilami.

$19 \alpha$. Lower valve, to slow the emarginate ear.

Tapes Staleyi.

Saxidonus gibbosus.

Fig. 20.

JANIRA BELLA.
59

58

PAGE

57

105 


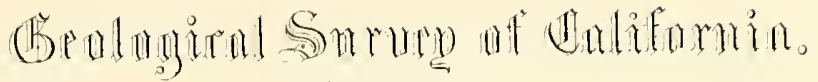
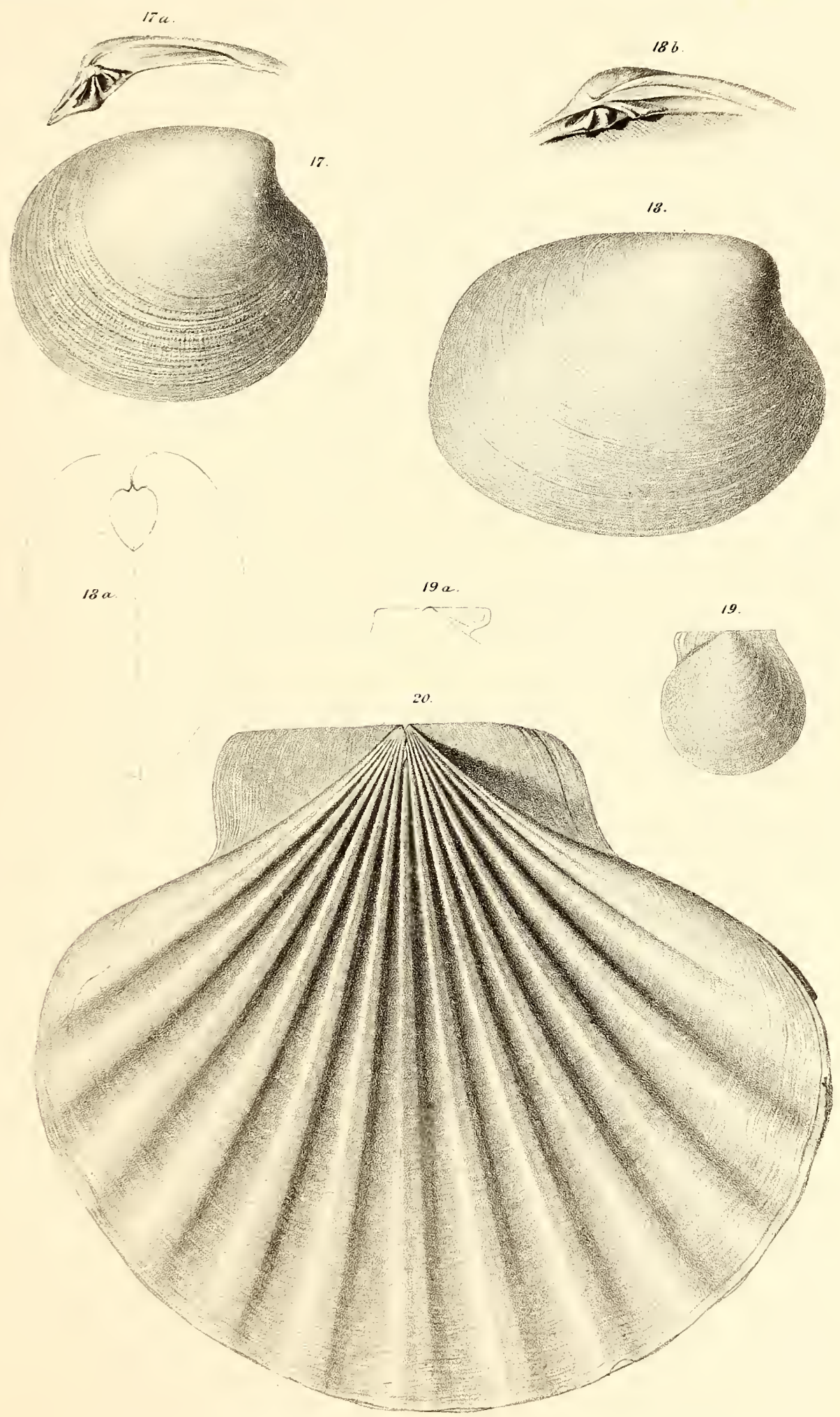




\section{PLATE XVII.}

Fig. 21.

Ostrea Veatchin.

PAGE

21 a. IIinge. 


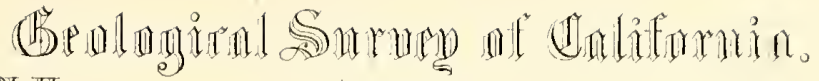

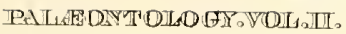
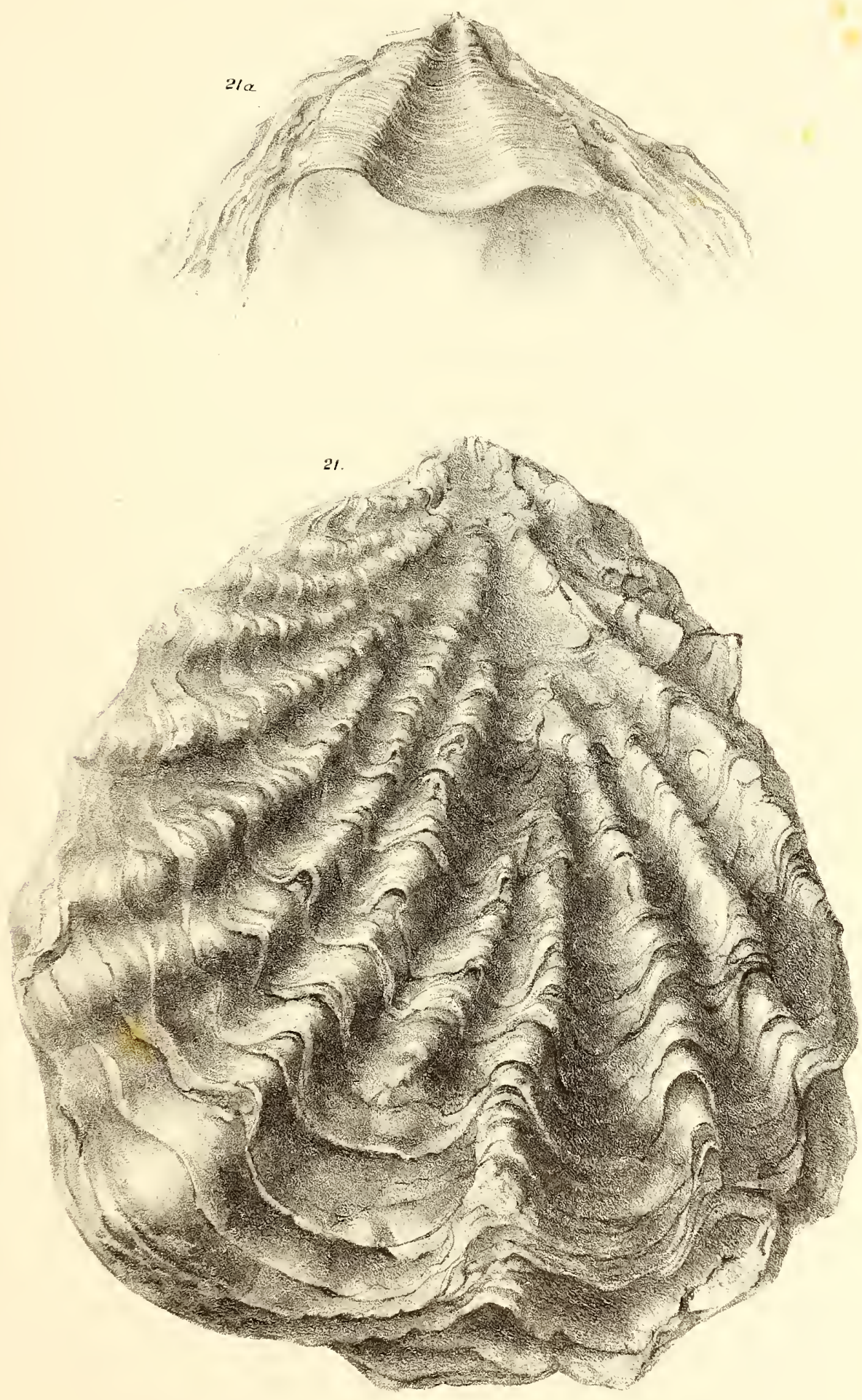




\section{PLATE XVIII.}

Fig. 22.

TAMIOSOMa gRegaria.

22 $u$. View of Mr. Conrad's original specimen, showing the internal plates.

226 . End view of the same, showing the thickness of the walls and the surface of the septa.

22 c. Magnified view of a section of the walls and of the origins of the transverse septa.

$22 d$. Magnified view of a portion of the internal surface with the remains of septa.

$22 \varepsilon$. Section of the wall of Radiolites SQUAMOSA, to exhibit the similarity of the columnar structure. 


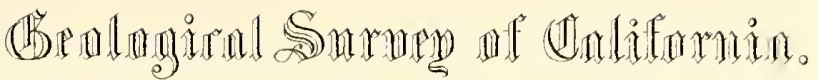

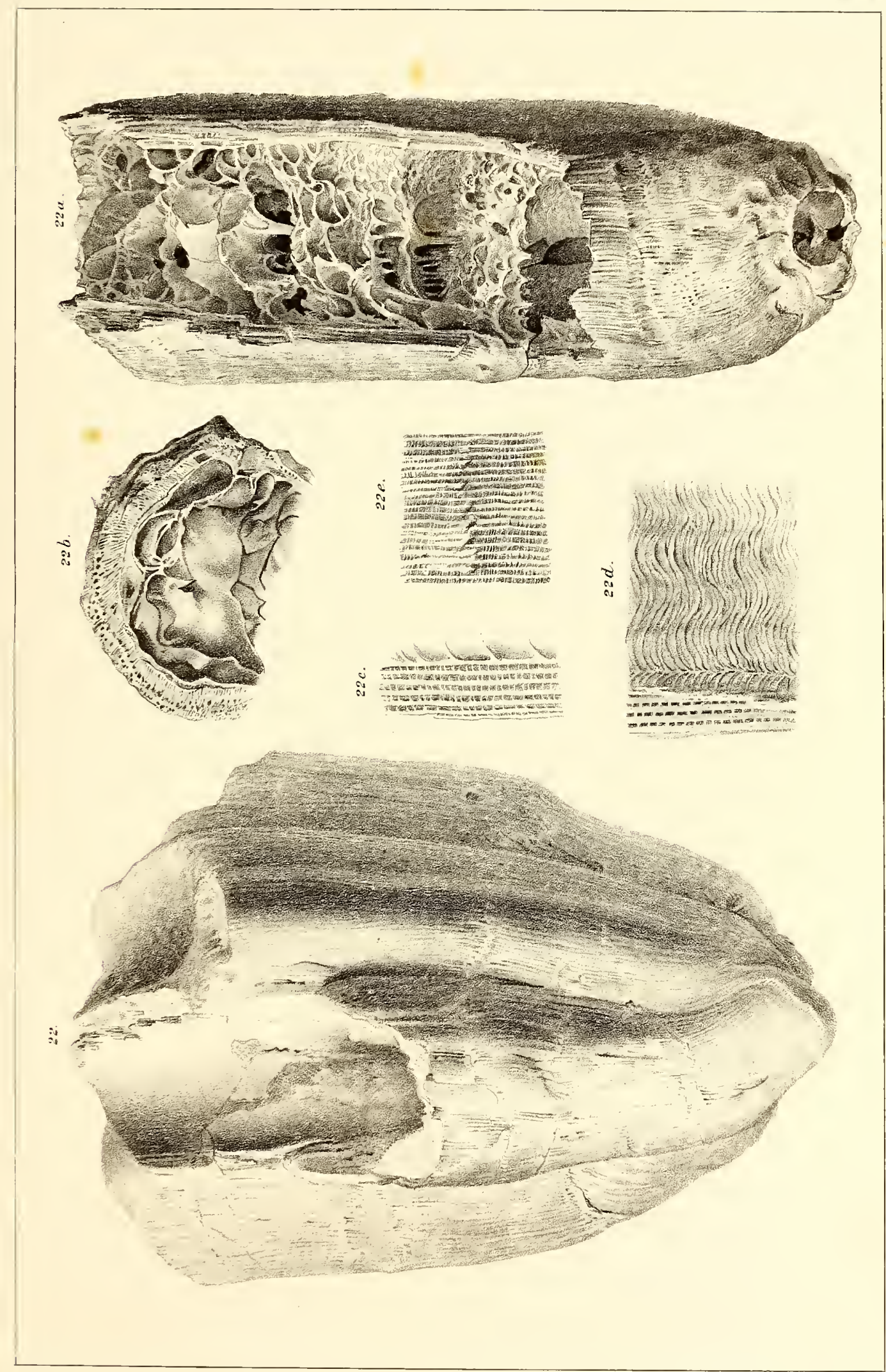






\section{PLATE XIX.}

Fig. 1.

Fig. $2 a-f$.

Fig. 3.

Fig. 4.
Crustacean. Indet.

Crustacean claws. Indet.

Callianassa Stimiponit.

Ptilotevthis foliates.
PAGE

127

127

127

128

130

Fig. 6. $6 a$.

A. Brewerir. Young.

Fig. 7 .

A. Triskir. Young.

7 u. Outline of body volution.

7b. Still younger specimen. 


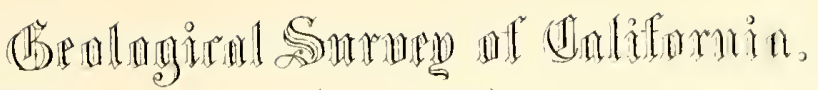

PAI ARDNT DLO WXVDU AIT. (Crelareous.)

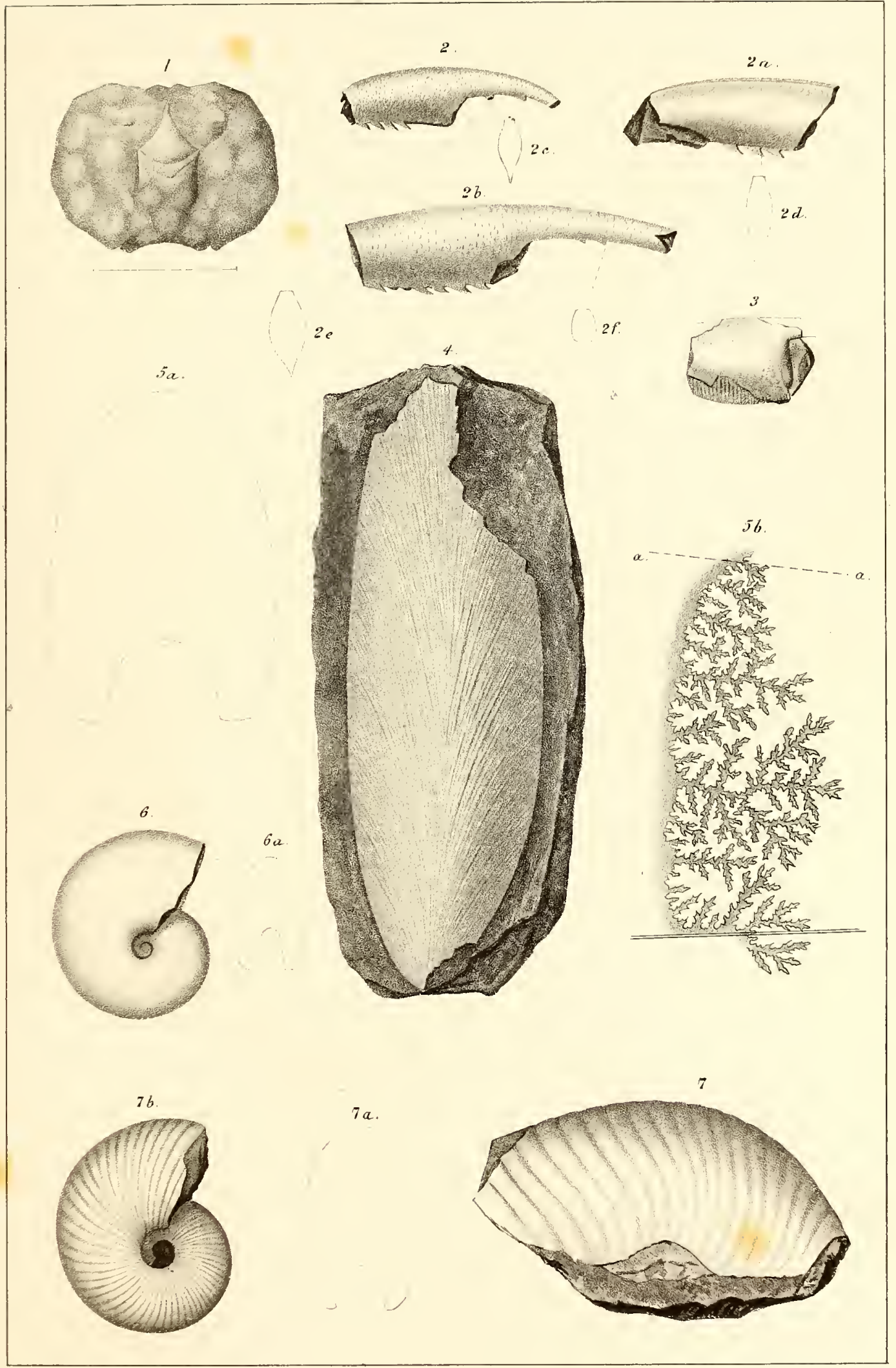






\section{PLA TE X X.}

Fig. 5.

Fig. 8 .
Amonites Breweri.

A. HoffmanniI. Variety.
PAGE

130

131

$8 a$. Front riew of the same.

Fig. $9 . \quad$ A. Batesir. Angulated variety.

132

$9 a$. Front view of the same. 


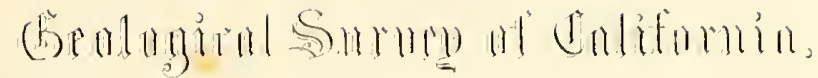

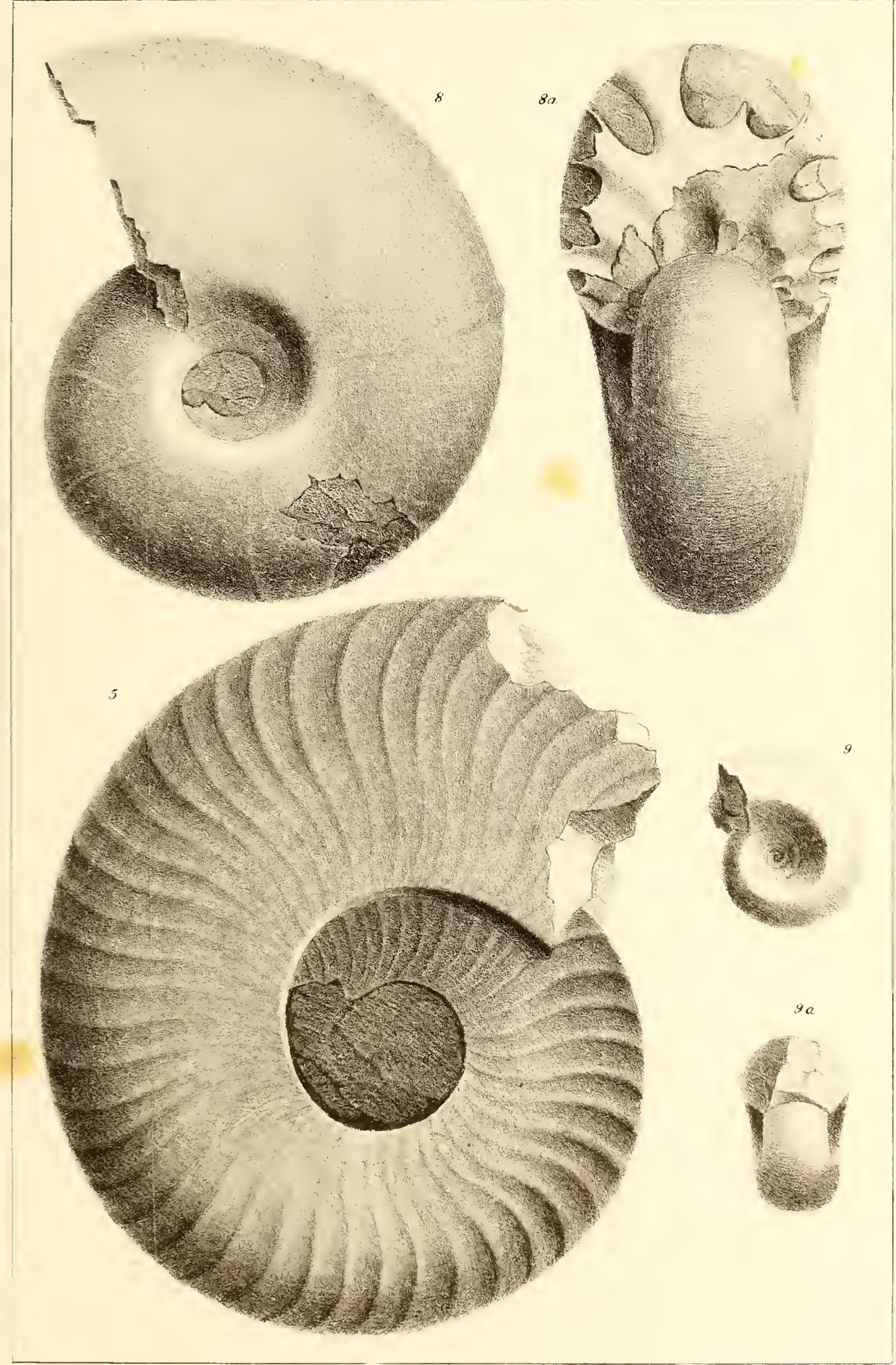






\section{PLATE XXI.}

PAGE

Fig. 10. Ammonites Batesil. Showing the gradation from the angulated whorls of the young state of this variety, to the rounded whorls in the older shell.

10a. Cross section of shell.

10 b. Septum.

Fig. 11.

A. Suciaensis.

$11 a$. Cross section of body whorl.

$11 b$. Septum. 


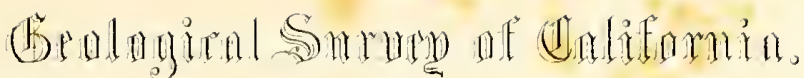

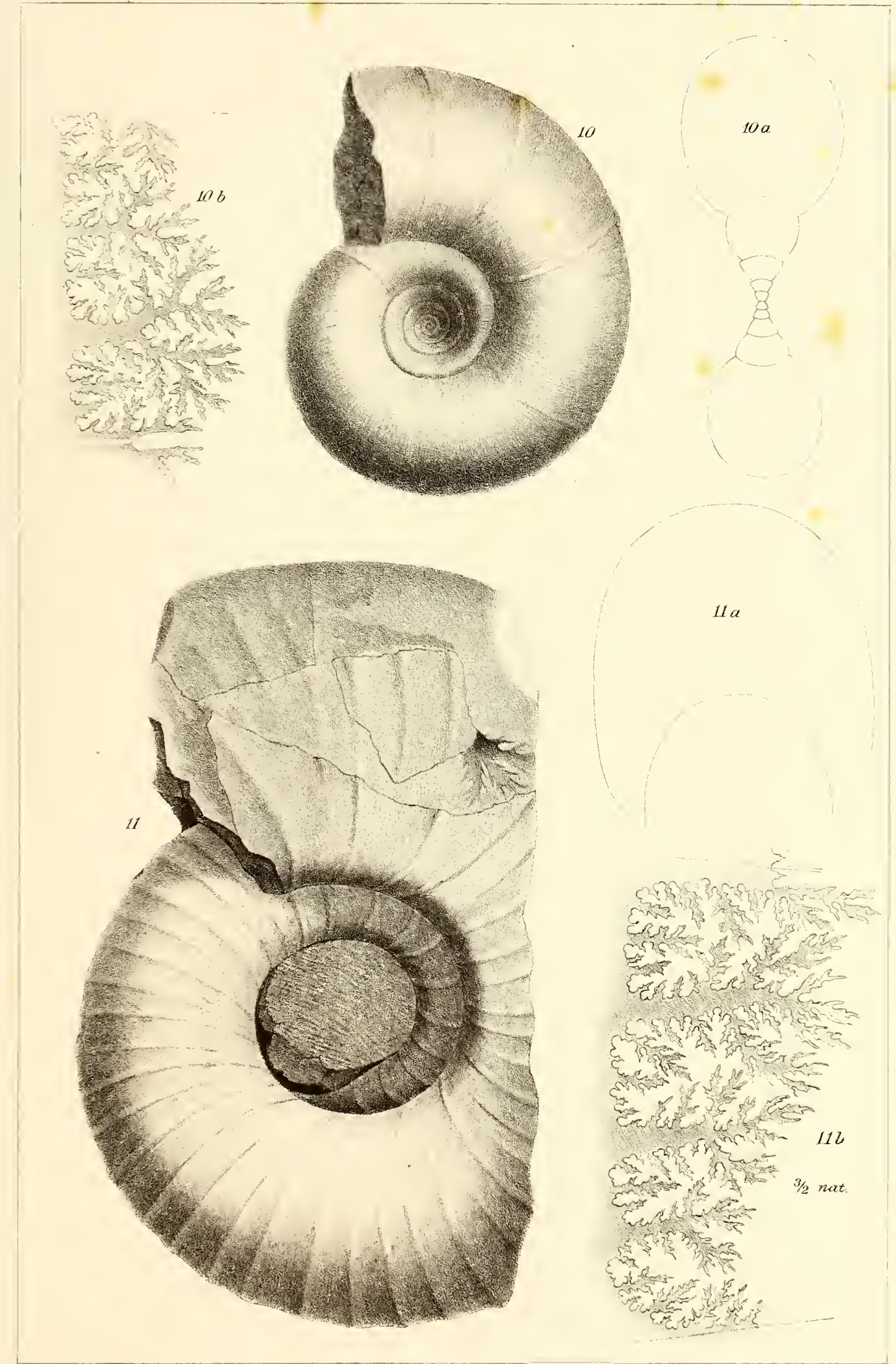






\section{PLATE XXII.}

Fig. 12. Ammonites JUGılis, from the Martinez Group at Benicia.

12a. Cross section of body whorl.

12b. Septum from same specimen.

Fig. 13. A specimen of the same from Martinez.

13 a. Front view of same.

Fig. 14.

A. Whiteley.

$14 a$. Front view of the same.

14b. Septum of the same. 


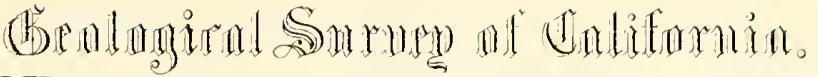
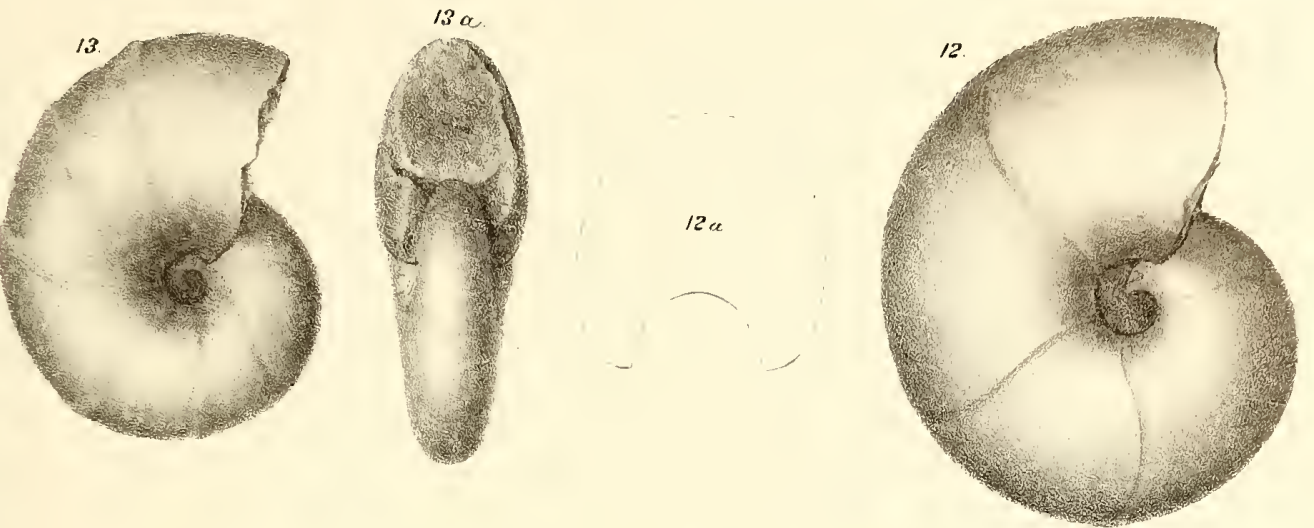

14-6.

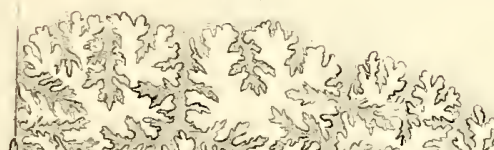

fिभ

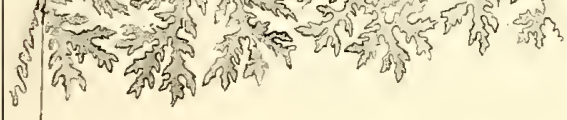

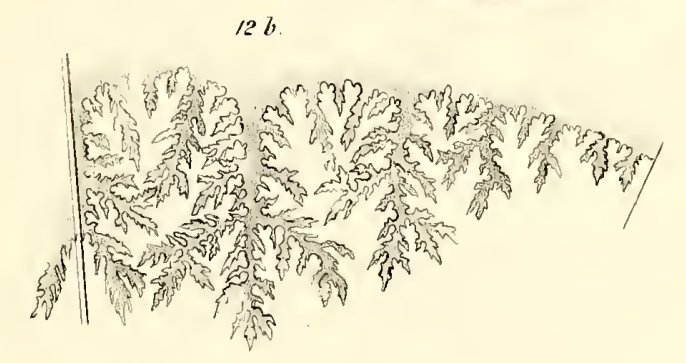

3.

1 (1)
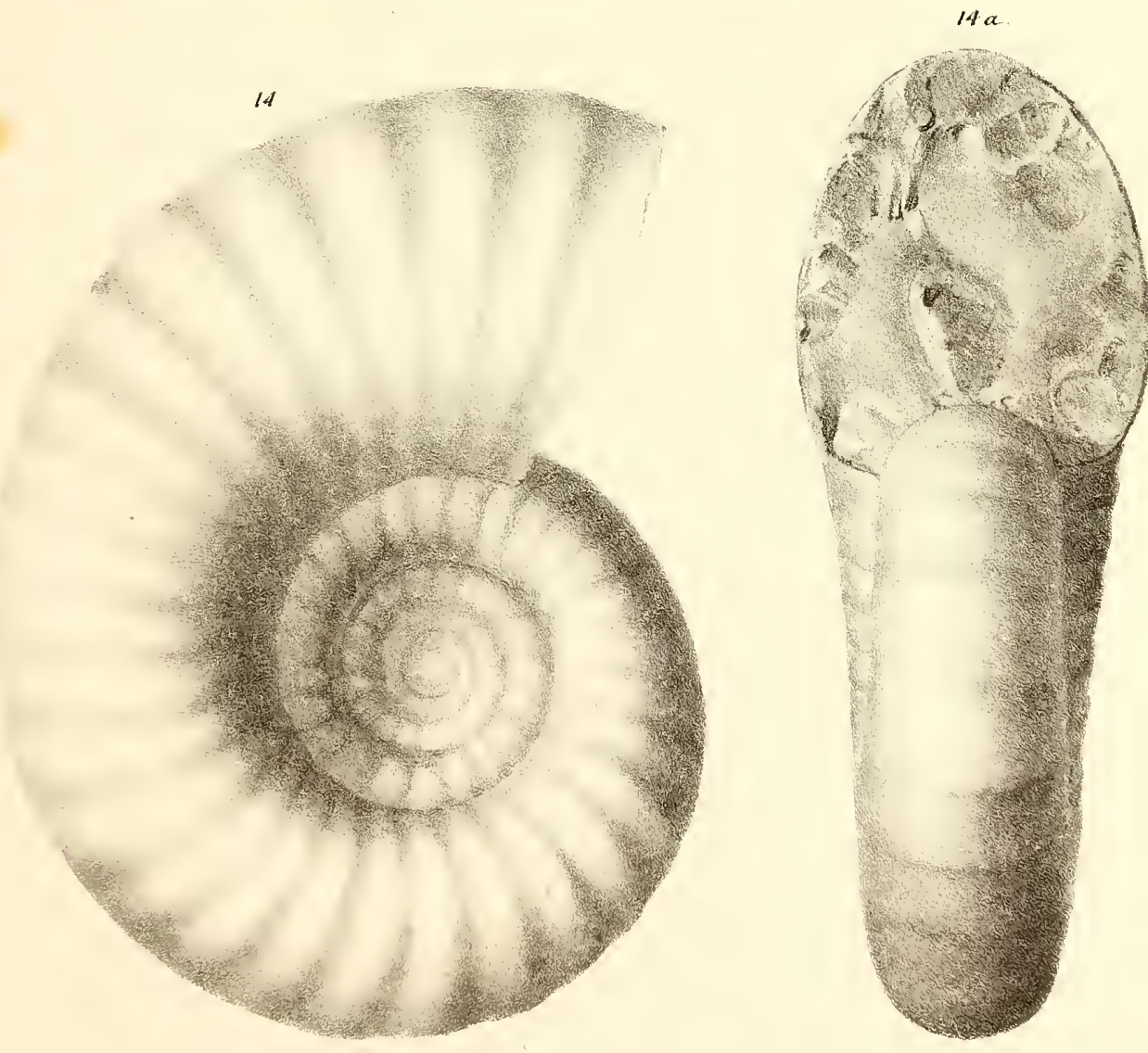




\section{PLATE XXIII.}

Fig. 15.

Amonites fraterieds.

PAGE

$15 a$. Cross section of last volution.

15b. Septum.

Fig. 16.

A. Stoliczkanus.

$16 a$. Front view of the same.

Fig. 17.

Septum of Axcyloceras Rérondi.

Fig. 15. ? Axcyloceras lineatus. Side view of straight limb.

$18 \alpha$. Dorsal view of same.

$18 \mathrm{~b}$. Cross section of same.

18 c. Septum. 


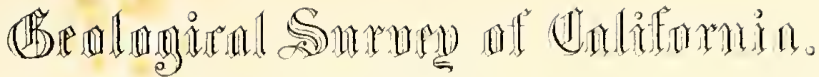

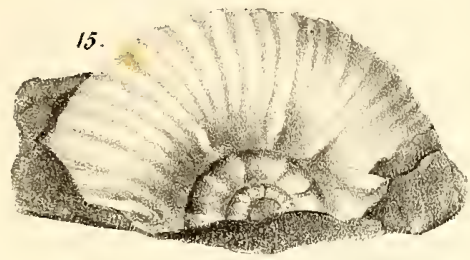

156.

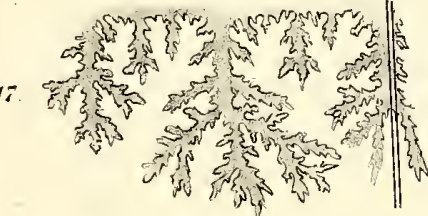

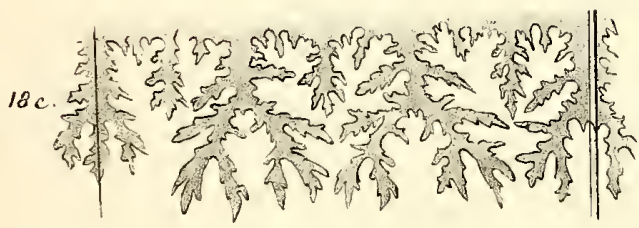
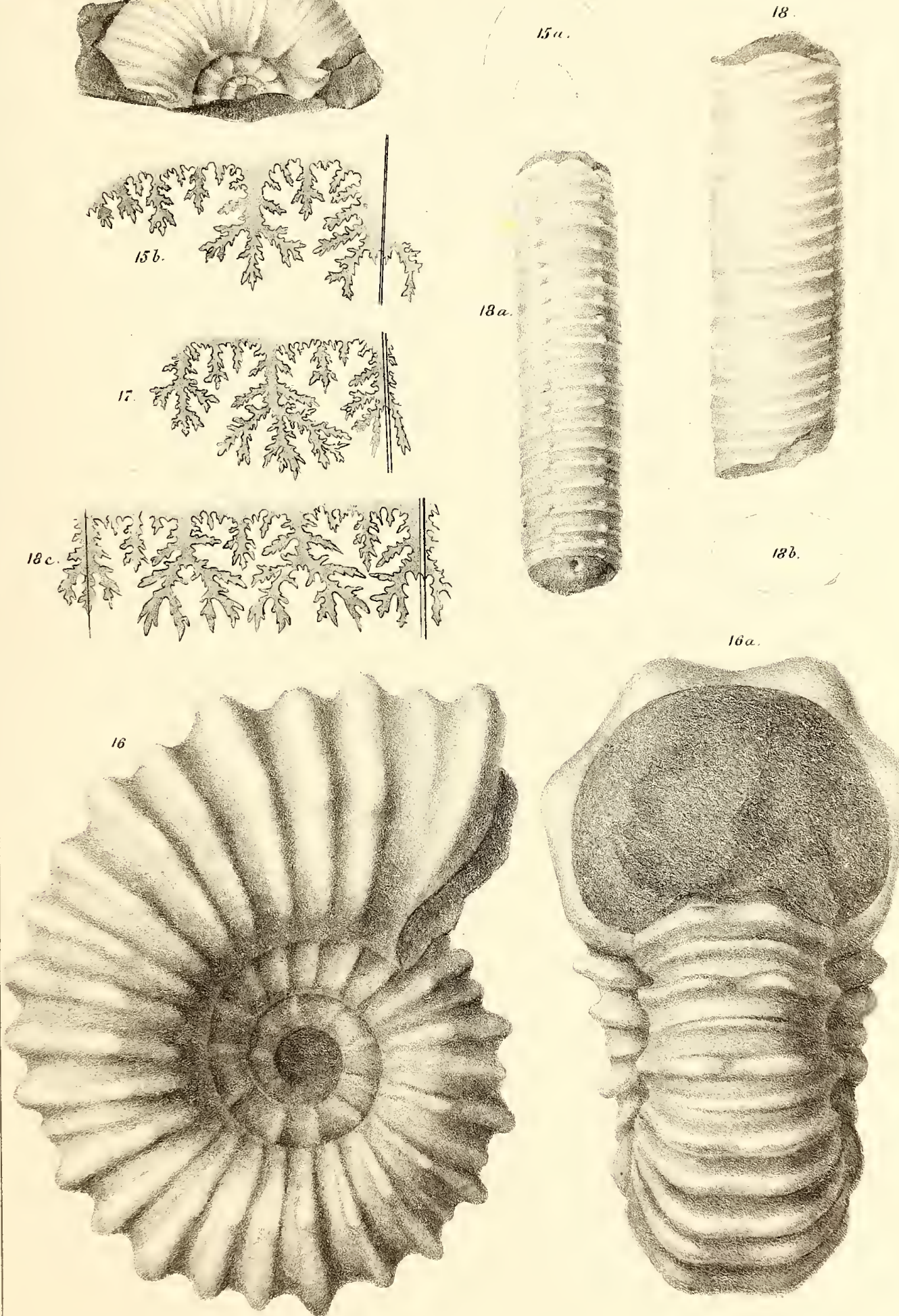




\section{PLA'TE XXIV.}

Fig. 19.
Axcyloceras percostatus.

PAGR

138 


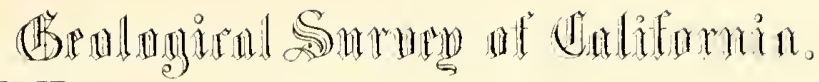

PALUFOTYPDLO GYOVDU ज्ञा.

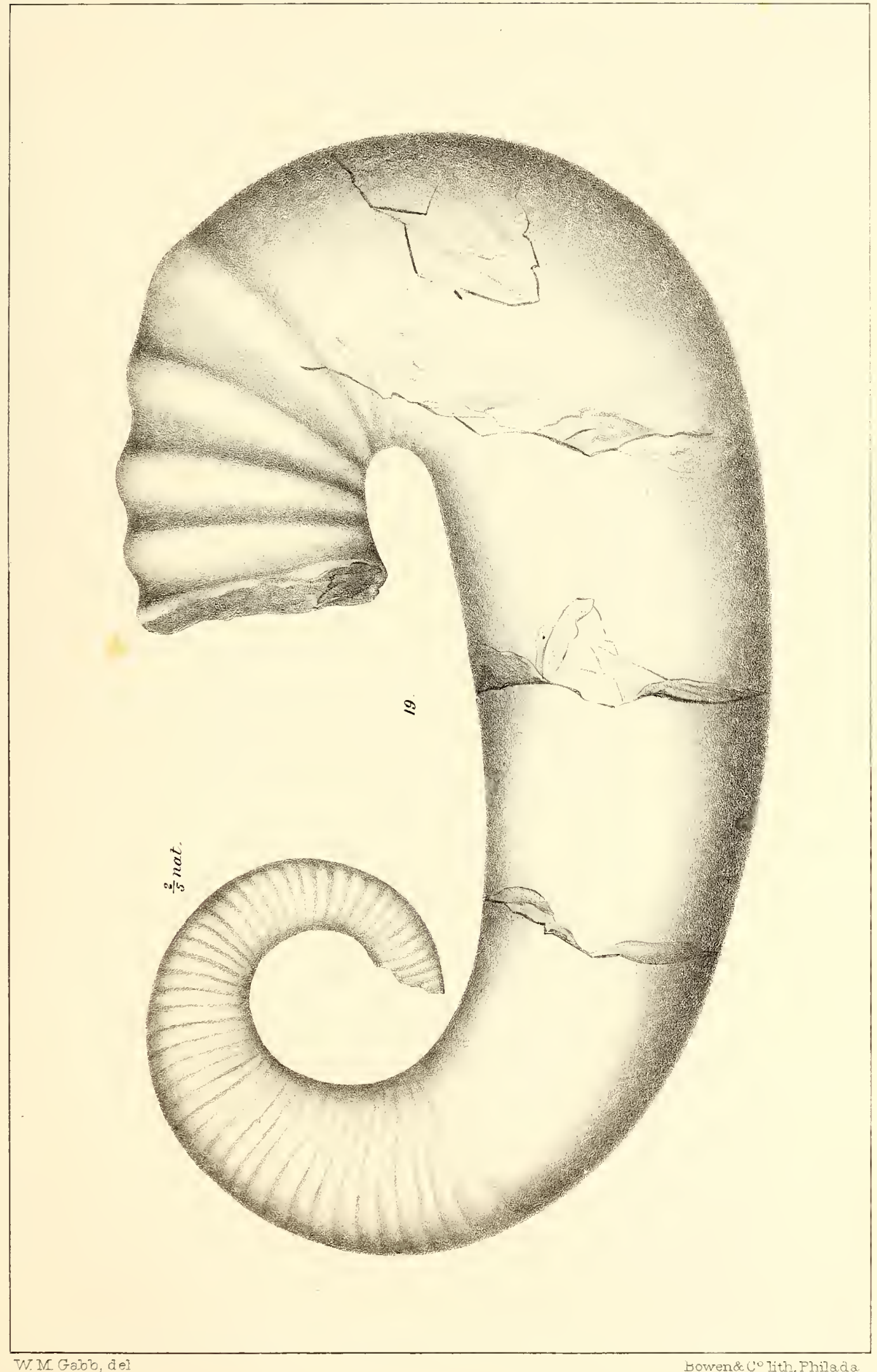






\section{PLATE XXV.}

Fig. 20. Helicancylus equicostatus. Top view of the spiral.

$20 \alpha$. Front view of the spiral.

$20 b, c, d$. Three views of the straight limb.

20 e. Side view of the largest limb.

$20 \mathrm{f}$. Cross section of aperture.

$20 \mathrm{~g}$. Septum.

Fig. 2 I.

DIPTYCHOCERAS LEVIS.

144

$21 a$. Cross section of the two smaller limbs, at the point of fracture of the specimen.

21 b. Septum. 


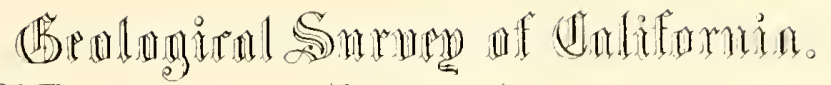

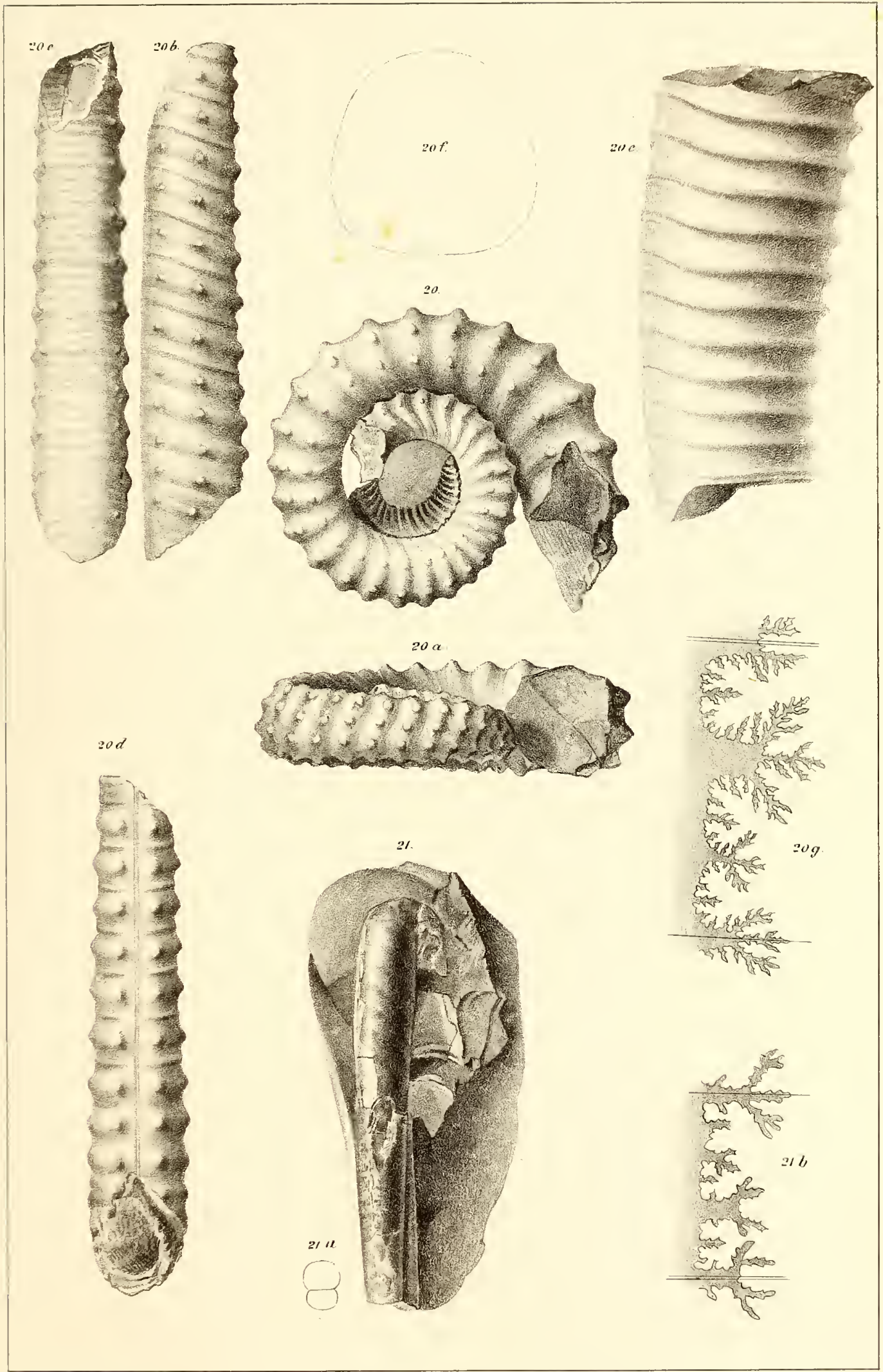






\section{PLATE XXVI.}

Fig. 22.

Fig. 23.

Fig. 24.

Fig. 25.

Fig. 26.

Fig. 27.

Fig. 28.

Fig. 29.

Fig. 30 .
Fusus rumidus. Magnified.

F. occidentalis. Magnified.

Neptunea (Tritonofusus) cretacea.

N. mucronata. Natural size.

Paleatractus crassus. Magnified.

Surcula preattenuata. Natural size.

S. (Surculites) sinuata. Natural size.

S. (Sunculites) inconsficua. Magnified.

Heteroterma trochoidea. Magnified. 


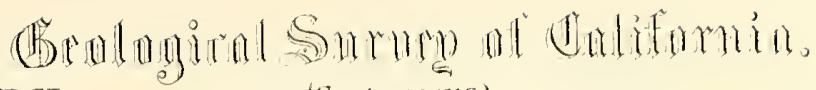
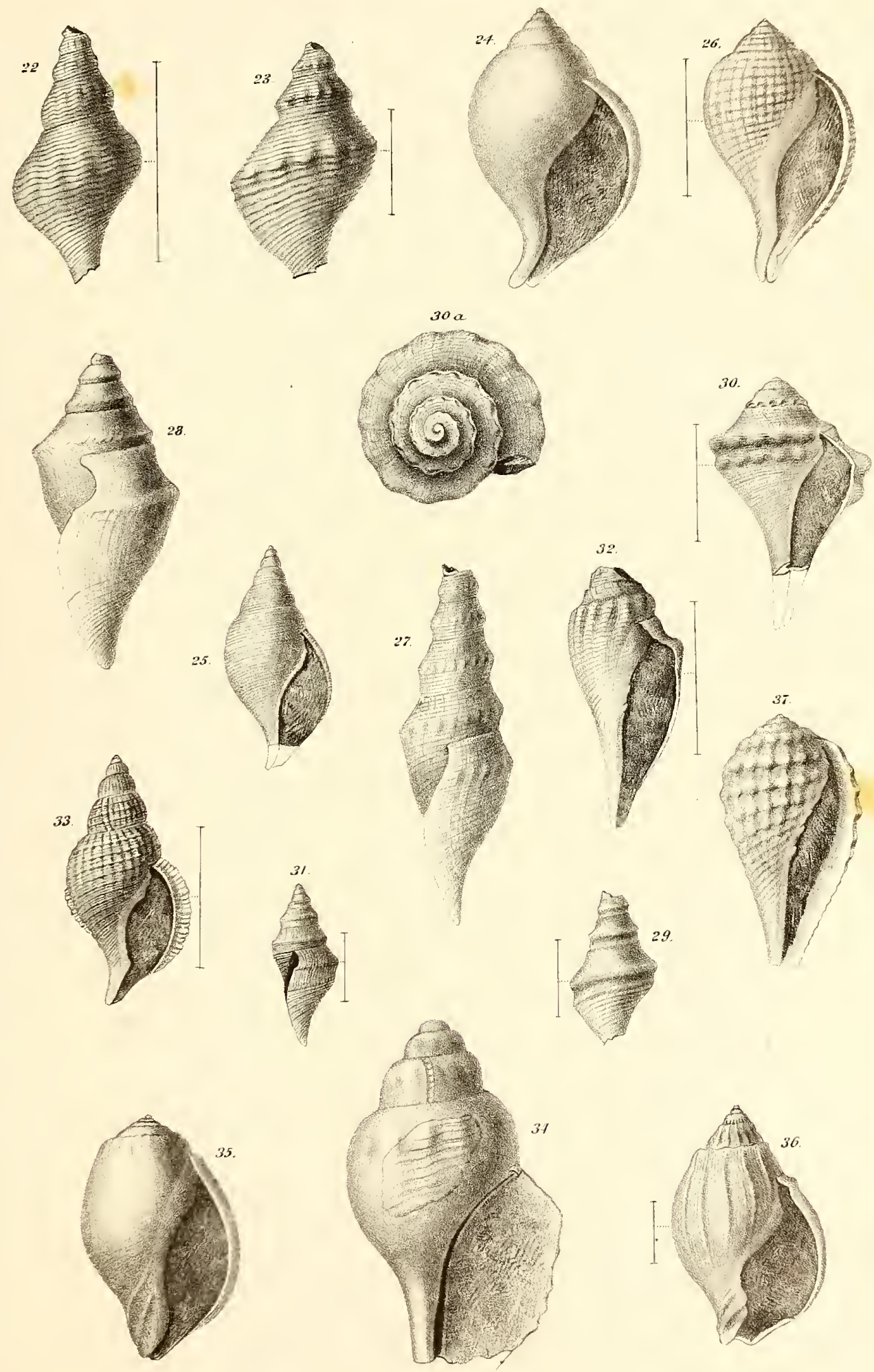




\section{PLATE X X VII.}

Fig. 38 .

Urosyca caudata. Natural size.

Figr. 39.

Neverita globosa. Magnified.

Fig. 40.

Ampullina striata. Magnified.

Fig. 41.

Terebra Califorica. Magnified.

Fig. 42 .

Pugrellus hanelus. Natural size.

Front view of an old and very heavily incrusted individual.

42a. Lateral view of the same.

Fig. 43. Cyprea (Luponia) Bayerquei. Side view.

$43 \alpha$. Top view.

$43 \mathrm{~b}$. View of the base.

43 c. A young specimen from Tejon.

Fig. 44. Cyprea (Epona) Mathewsonit. Magnified. Top view.

$44 a$. Side view.

$44 b$. View of the base.

Fig. 45 .

Anchura transversa. Magnified.

Fig. 47.

Helicaulax bicarinata. Natural size. 


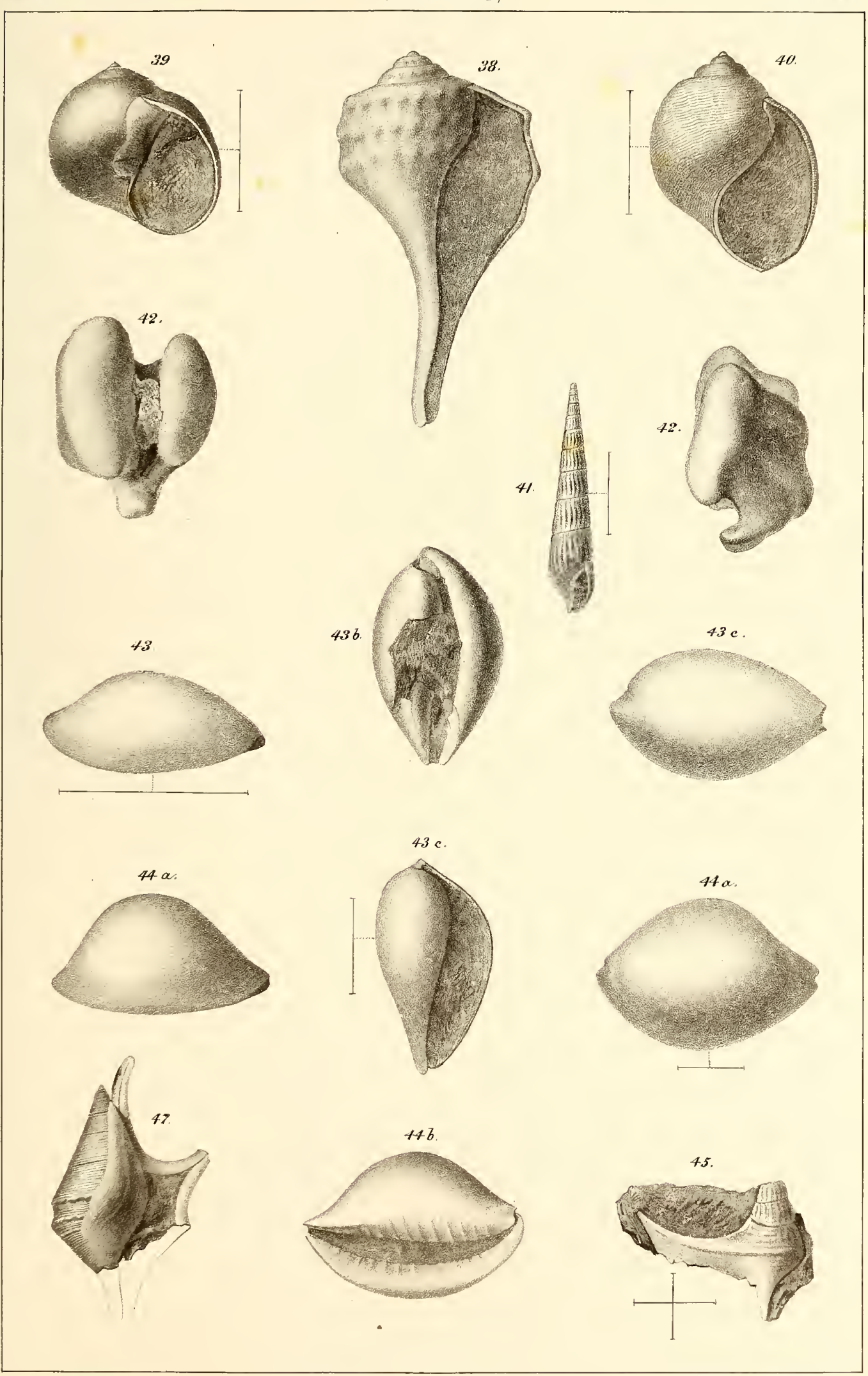

PIATHE 27 。 




\section{PLATE XX VIII.}

Fig. 46.

Fig. 48 .

Fig. 49.

Fig. 50.

Fig. 51.

Fig. 52.
? ANChura Carinifera. Magnified.

Helicadlax costata. Magnified.

Loxotrema tUrrita, Natural size.

Atresius liratus. Magnified.

Turritella Martinezexsis. Natural size.

Nerita (Theliostyla) triangulata.

Magnified view of the back.
PAGE

166

167

168

169

159

170

52 a. Front view.

Fig. 53.

Calliostoma radiatum. Magnified.

170

Fig. 54.

Ataphrus crassus. Magnified.

171

Fig. 55.

Margaritella angulata. Magnified.

172

Fig. 56.

Acmea Tejonexsis. Magnified.

172

Fig. 57.

Acteonixa pupoides. Magnified.

173

Fig. 58.

Acteonella oviformis. Natural size.

173

Fig. 59.

Liocium puxctatum. Magnified.

174

Fig. 60 .
Ringinella polita. Magnified. 


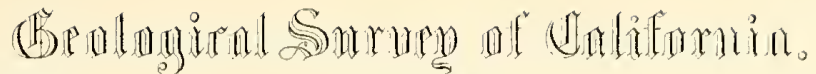

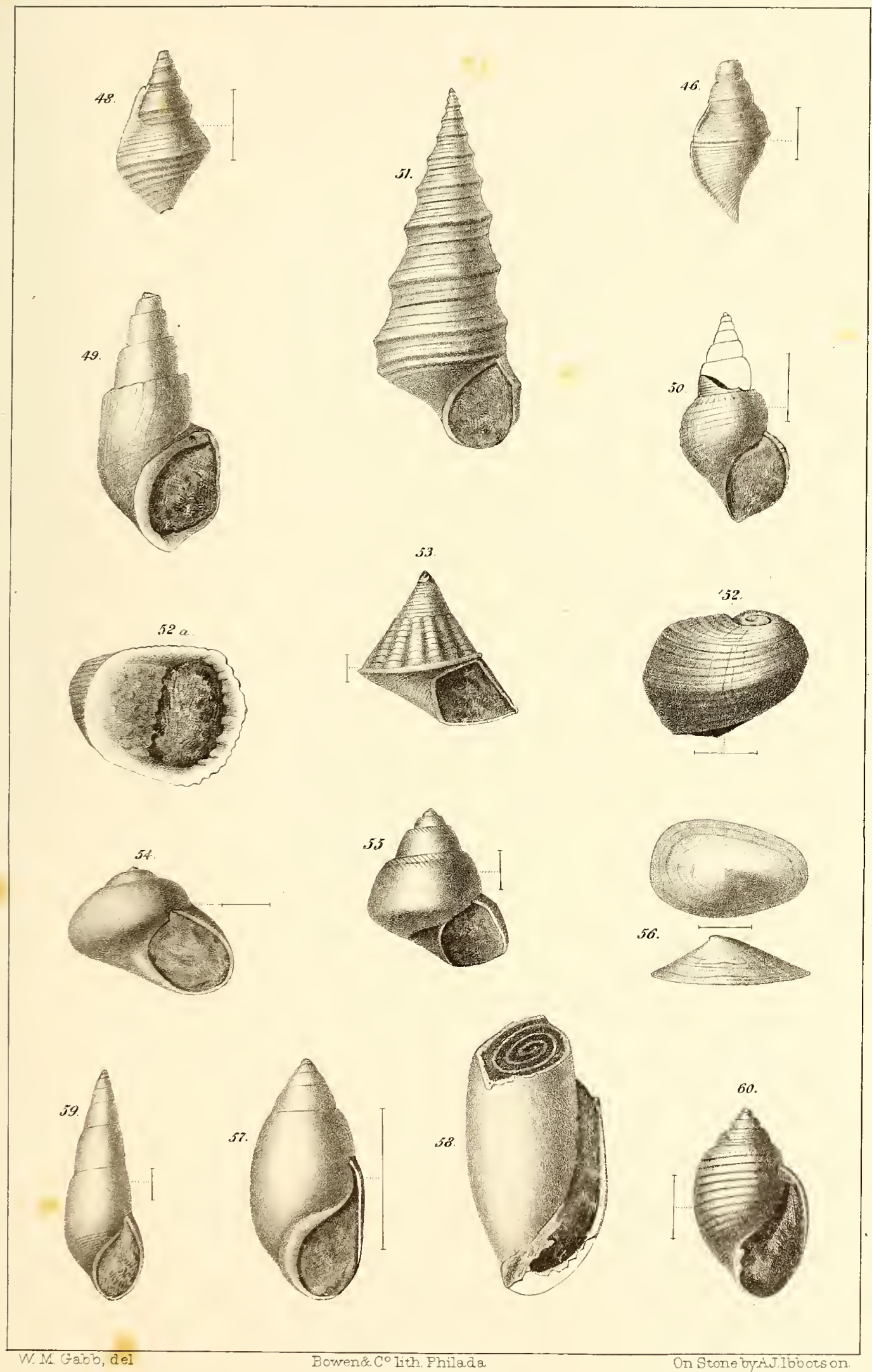






\section{PLATE X XIX.}

Fig. 61.

Solen (Hypogella) cuneatus. Natural size.

Corbula Hornit. Natural size.
PAGE

175

176

62. Left valve.

62a. Right valve.

62 b. Anterior end.

Fig. 63.

C. Alæforais. Natural size.

Fig. 61.

Anatina Quadrata. Natural size.

177

Fig. 65 .

Pholadomya Oregonexsis. Natural size.

178

Fig. 66.

Plecromya papyracea. Natural size.

178

Fig. 67 .

Arcomya undelata. Natural size.

179

Fig. 68.

? Mactra texussima. Natural size.

179

lig. 69. $69 a$.

Hinge of Cramophora Ashburneit.

180

69. Right valve. $69 a$. Left valve.

Fig. 70.

Asapilis multicostata. Magnified.

181

Fig. 71 .

Teluina Remondi. Natural size.

182

Fig. 73.

T. Equalis. Natural size. 


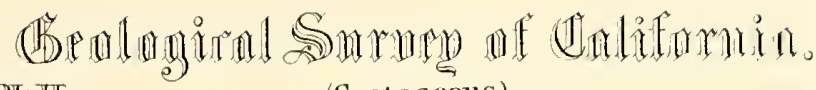

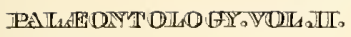
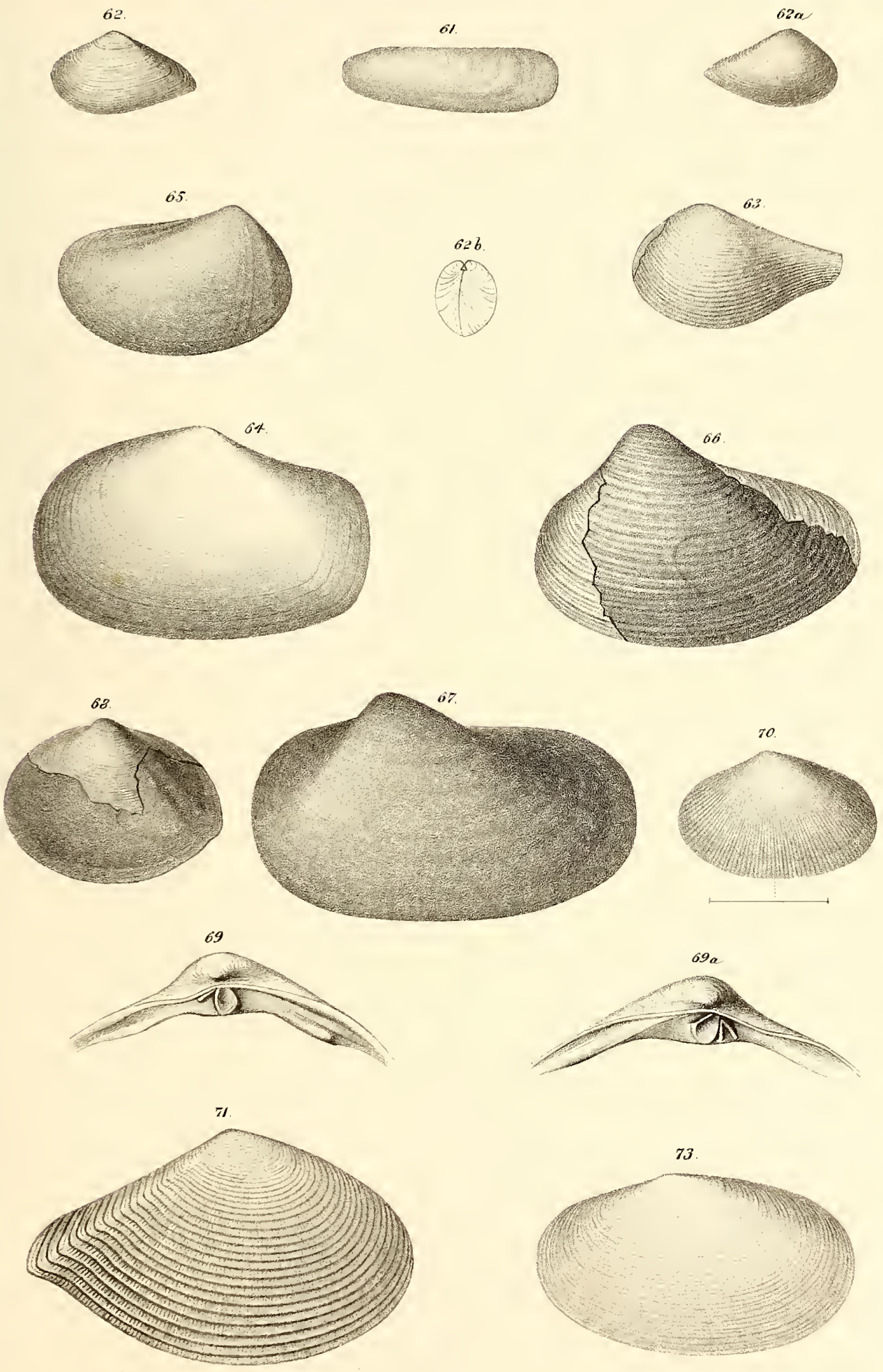




\section{PLATE XXX.}

Fig. 72.

Fig. 74.

Fig. 75.

Fig. 76.

Fig. 77 .

Fig. 78.

Fig. 79 .

Fig. 80 .
Teldica Hoffmanniana. Natural size.

? T. Undulffera. Natural size.

Dorax Latus. Natural size.

Venus fquilateralis. Natural size.

? Meretrix fragilis, Natural size.

M. Hornil. Natural size.

Caryatis nitida. Natural size.

? Thetis elongata. Magnified.
$80 a$. End view.

Fig. 81. Cardium (Levicardium) annulatum. Natural size.

Fig. 82.

C. (Protocardium) translucidum. Magnified.

Fig. 83. Section of ribs of Cardita Holixi when perfect.

$83 \alpha$. The same when weathered.

83 b. Section of ribs of C. PLANicosta for comparison.

Fig. 84.

Hinge of Clisocolus dubius. Right valve.

Fig. 85 .

Crassatella compacta. Natural size.

Fig. 86.

Unio Hubbaidil. Natural size.

situ. End view. 


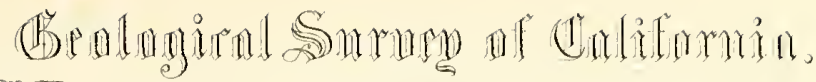

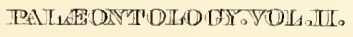

(Cretaceous.)

PTA THE








\section{PLATE XXXI.}

Fig. 87.

Fig. 88 .

Fig. 89 .

Fig. 90 .
Mrtilus Quadratus. Natural size.

Modiola majon. $\Lambda$ rather small specimen.

Meleagrina antigua, Natural size.

Inoceramus Ellotif. A cast.
PAGE 191

191

192

193

$90 \mathrm{a}$. Form of the ribs from the impressions in the matrix. 


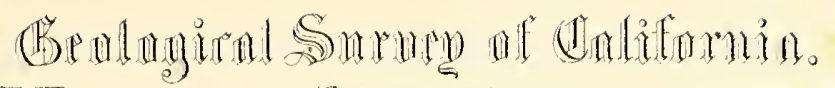
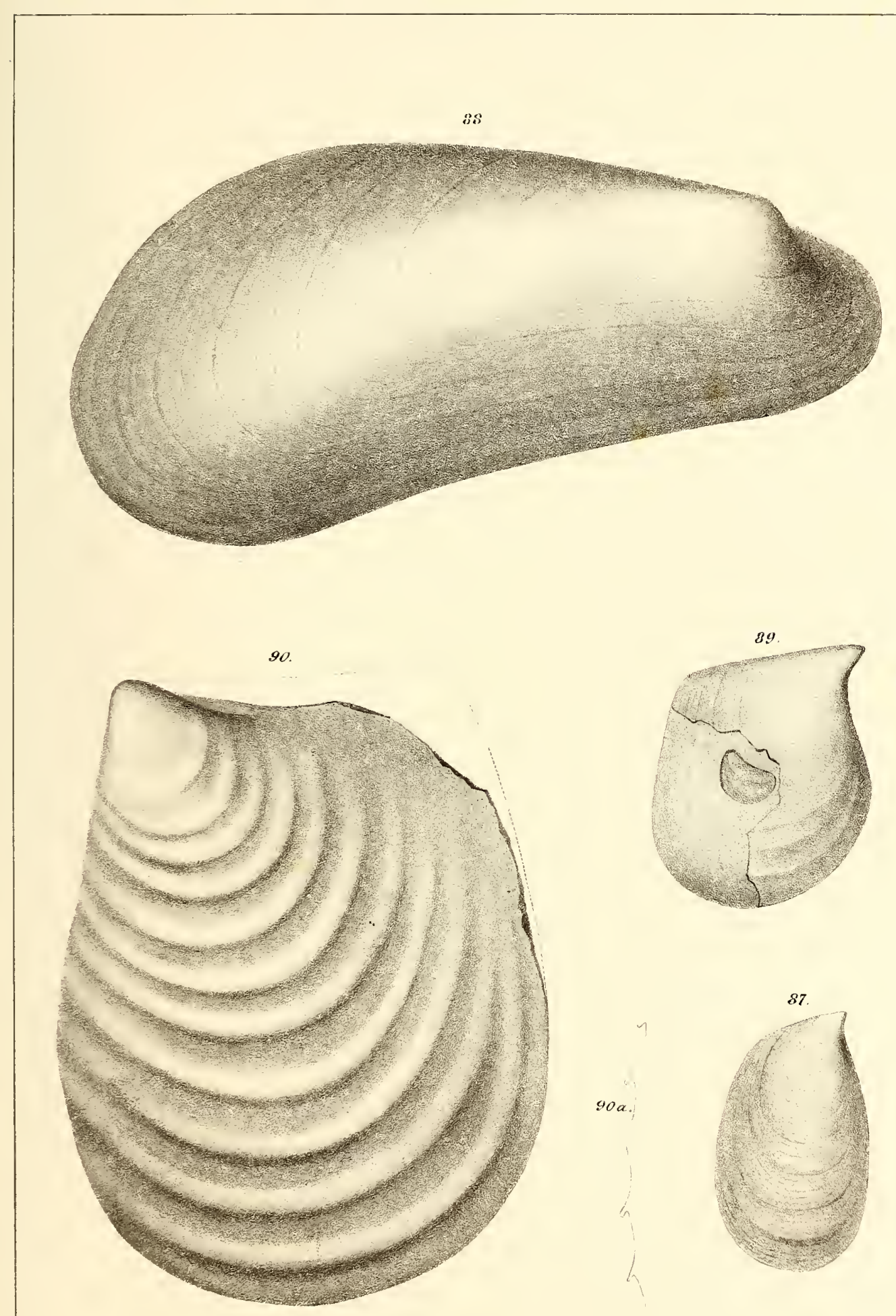

$90 a$. 




\section{PLATE XXXII.}

Fig. 91.

Iroceramus Whitneyi. From a cast.

PAGE

Fig. 92.

Aucella Piochin. Anterior view. Natural size.

193

194

$92 a$. Back view of large valve.

$92 b$. Small valve of a smaller individual.

92 c. View of interior of the small valve of a young individual, about twice natural size.

Fig. 93 .

Pinca Bueweril. Natural size.

Fig. 91.

Nucula solitaria. Magnified.

Fig. 95. Magnified view of the surface of Pectex Traskir, from a specimen from Vancouver Island. 


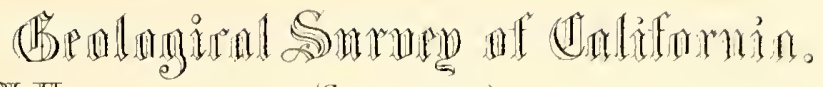

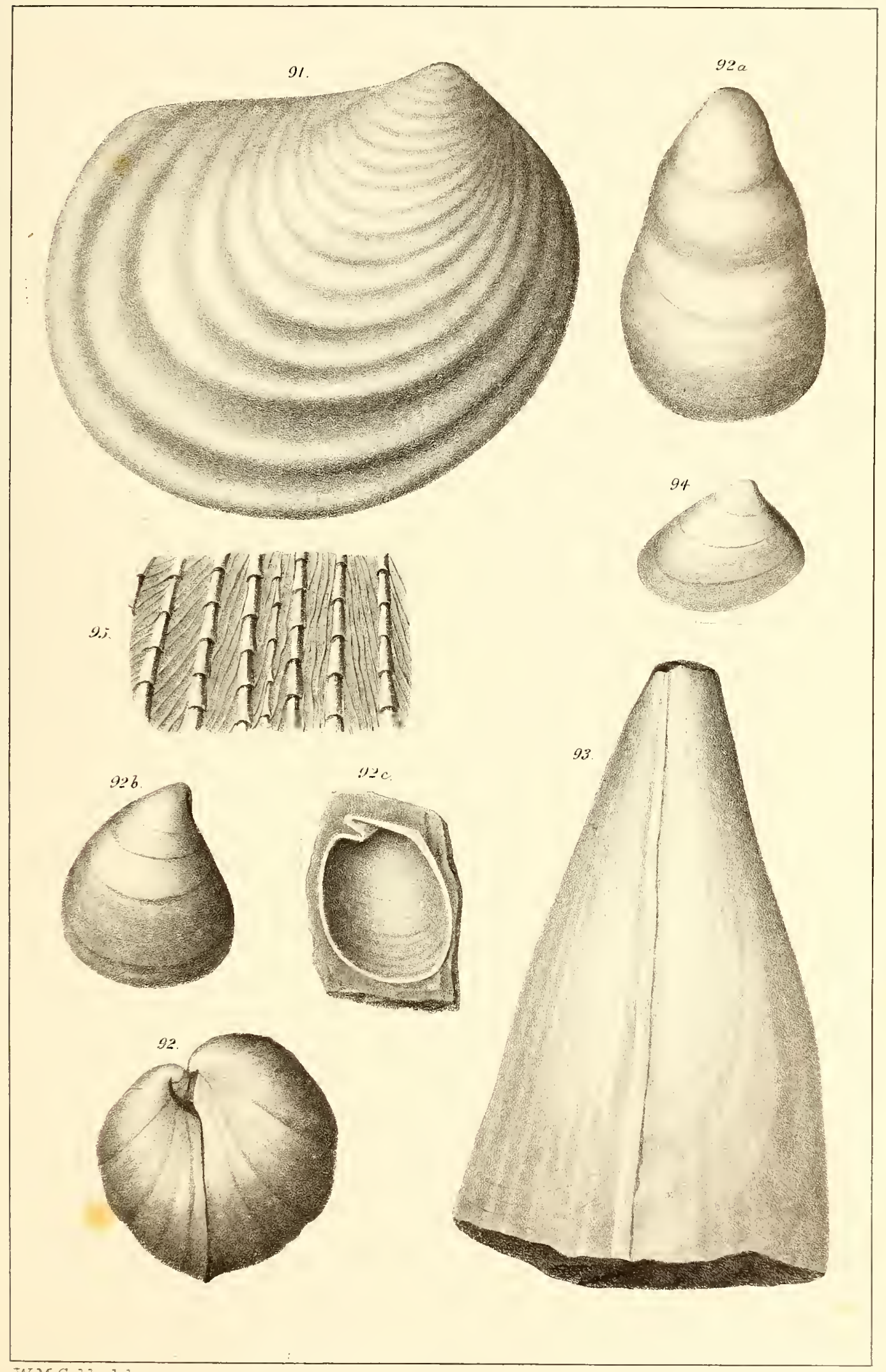






\section{PLATE XXXIII.}

Fig. 96 .

Pecten Malinezensis. Magnified.

PAGE

Fig. 97.

P. Complexicosta. Natural size.

198

199

$97 a$. Magnified view of the surface.

Fig. 98. $98 a$.

P. inter-radita. Natural size.

199

Fig. 99.

NeItuea GRAxDicosta. Magnified.

200

99 a. Section of the ribs.

Fig. 100.

Lima Shastaensis. Magnified.

201

Fig. 101.

L. mutimadita. Natural size.

201

Fig. 102.

Axomia Vaxcouverexsis. Natural size.

202

Fig. $103 b, c, d$.

Ostrea Idraessis. Young.

203 


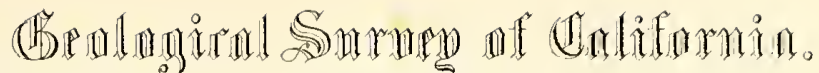

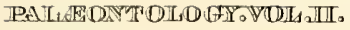
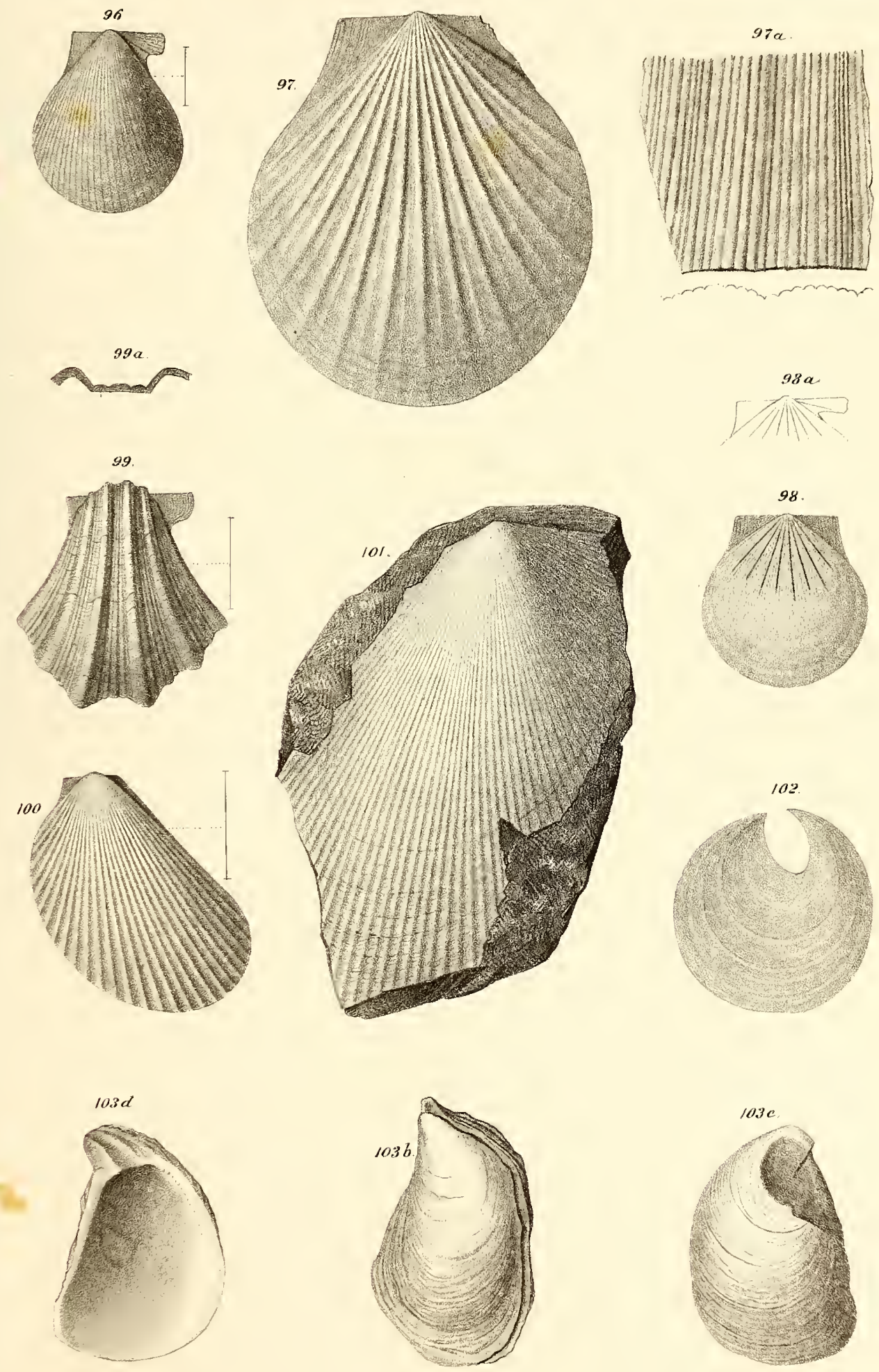



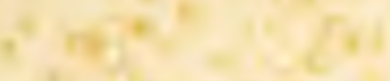

(20)

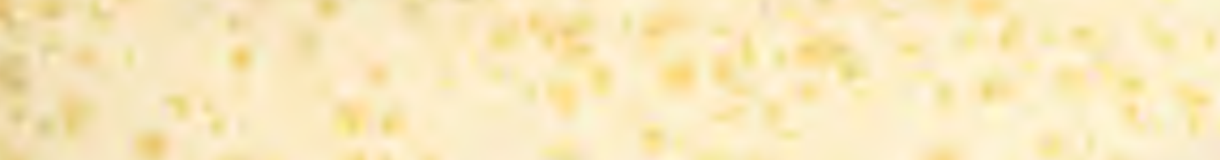

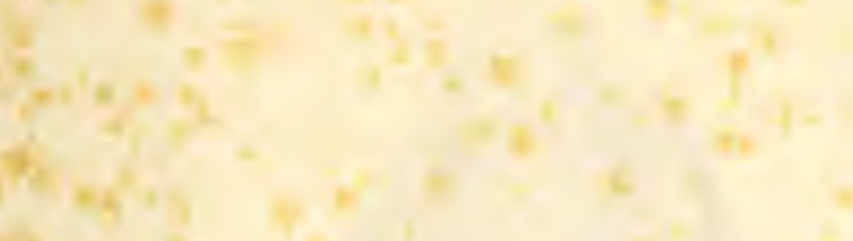

$\sqrt{2}$

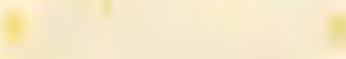

1

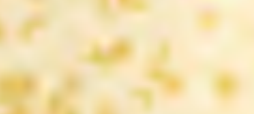

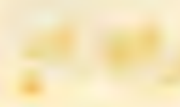

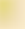
(an $=9$

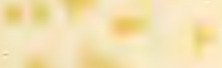

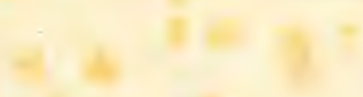

(12 


\section{PLATE XXXIV.}

Fig. 103. Ostrea Idriaensis. Natural size.

$103 a$. Hinge of lower valve from a very large specimen.

Fig. 104. Ostrea Appressa. Natural size.

$104 a$. Hinge.

Fig. 105. Rhychoselda Whitseyt. Natural size.

$105 a$. Sille view.

$105 b$. Outline of the ribs.

Fig. 106. 2 Smlotrochus curtus. Top view.

106 «. Longitudinal section. 


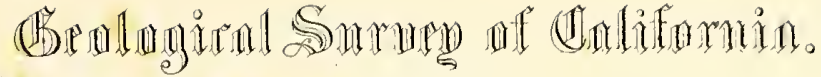

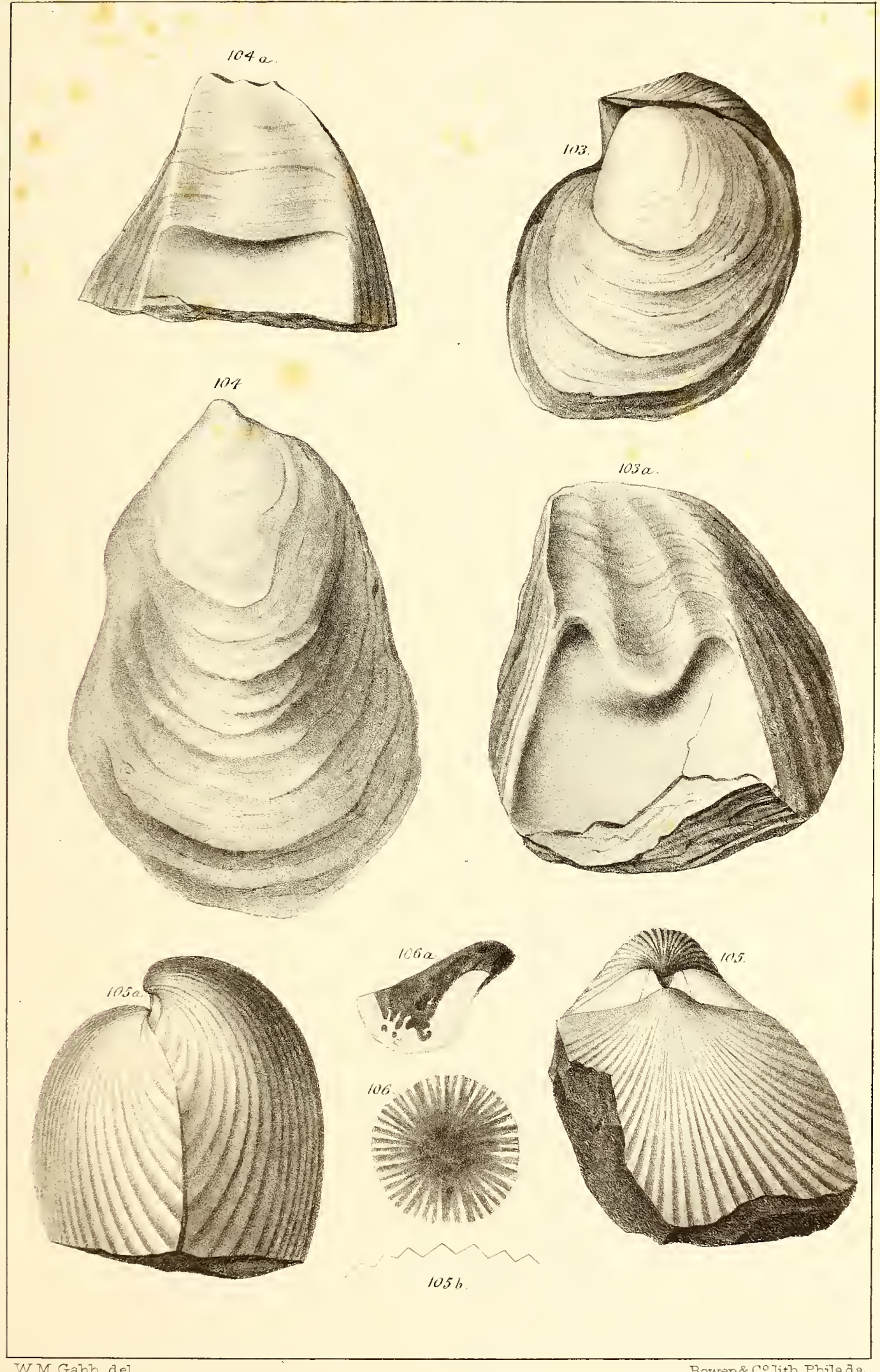

Bowend Conith Phitade 




\section{PLATE XXXV.}

Fig. 1. Amonites Pedernalis. Natural size. 258

1 a. Cross section showing the peculiar form of the dorsum.

Fig. 2.

Fusus Mexicanus. Magnified.

259

Fig. 3.

Lunatia Pederalis. Natural size.

259

Fig. 4.

Euspira tabulata. Natural size.

260

Fig. 5.

Chemintzia zebra. Natural size.

260

Showing the colored markings on the surface.

Fig. 6.

Trlostoma mutabilis. Natural size.

261

6 $a$. Magnified front view of a small specimen, showing the crenulated outer lip.

6b. Top view of the same.

6 c. Top view of an unusually large and rough specimen.

Fig. 7. Anchura monilifera. Magnified.

262

Fig. 8.

Cerithium Mexicanum. Magnified.

263

Fig. 9.

Angaria cingulata. Natural size.

264

Fig. 10. $10 a$.

Cixulia rectilabrum. Magnified.

264 


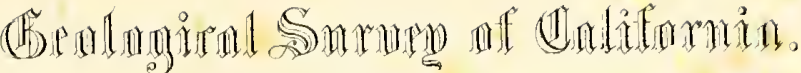

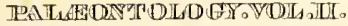
(Cretaceous.)

J

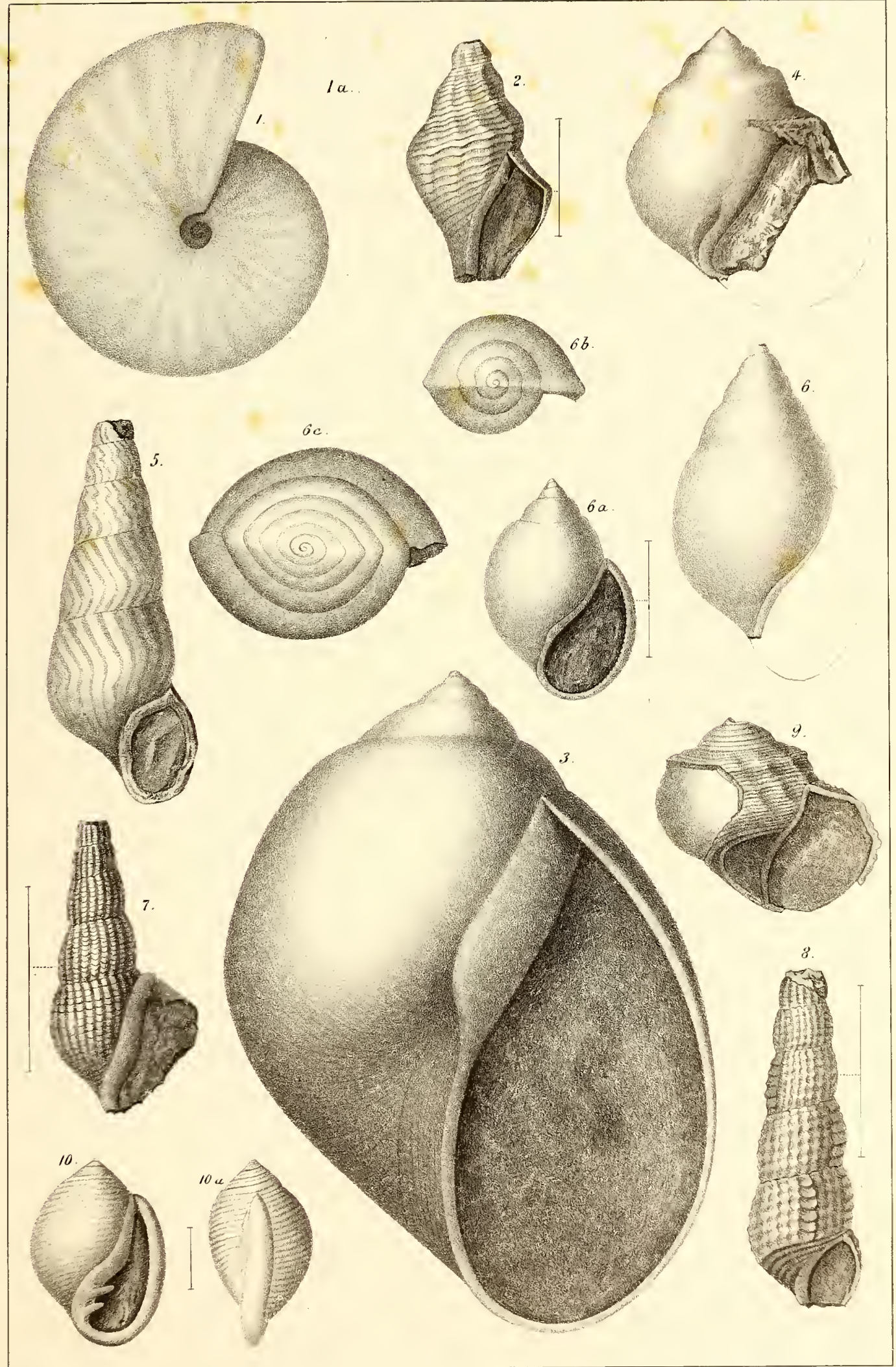

W.M. Gabb, del 




\section{PLATE XXXVI.}

Fig. 11.

UNDETERIINED.

PAGE

Fig. 12.

Pholadoniya Soxorensis. Slightly magnified.

276

Fig. 13.

Tapes Hilgardi. Natural size.

265

Fig. 14. Cardium (Graxocardium) sabulosum. Natural size.

Fig. 15. Cardium (Protocardium) granuliferum. Natural size.

Fig. 16.

? Cardita alticosta. Magnified.

267

Fig. 17.

Rejonda Furcata. Natural size.

17 a. Hinge of left valve.

Fig. 18. Cucullea inermis. Natural size.

$18 a$. End view of same.

Fig. 19. Exogrra Plicata. Natural size.

$19 a$. Edge view of same. 


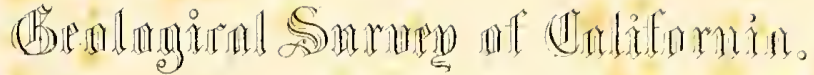

TEATARDNT OLDGY, VOI, III. (Cretaceous.)

PTARTES B

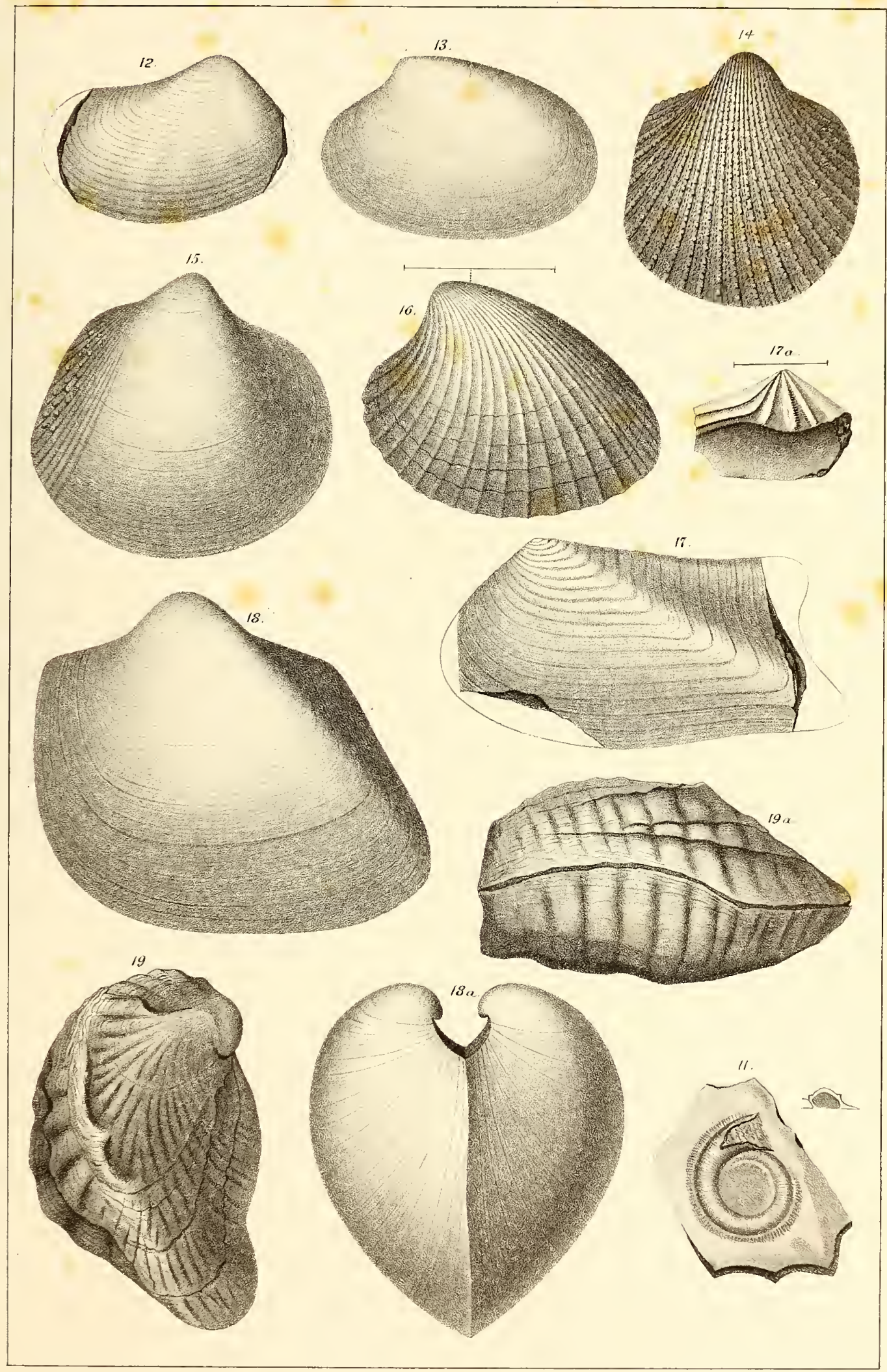








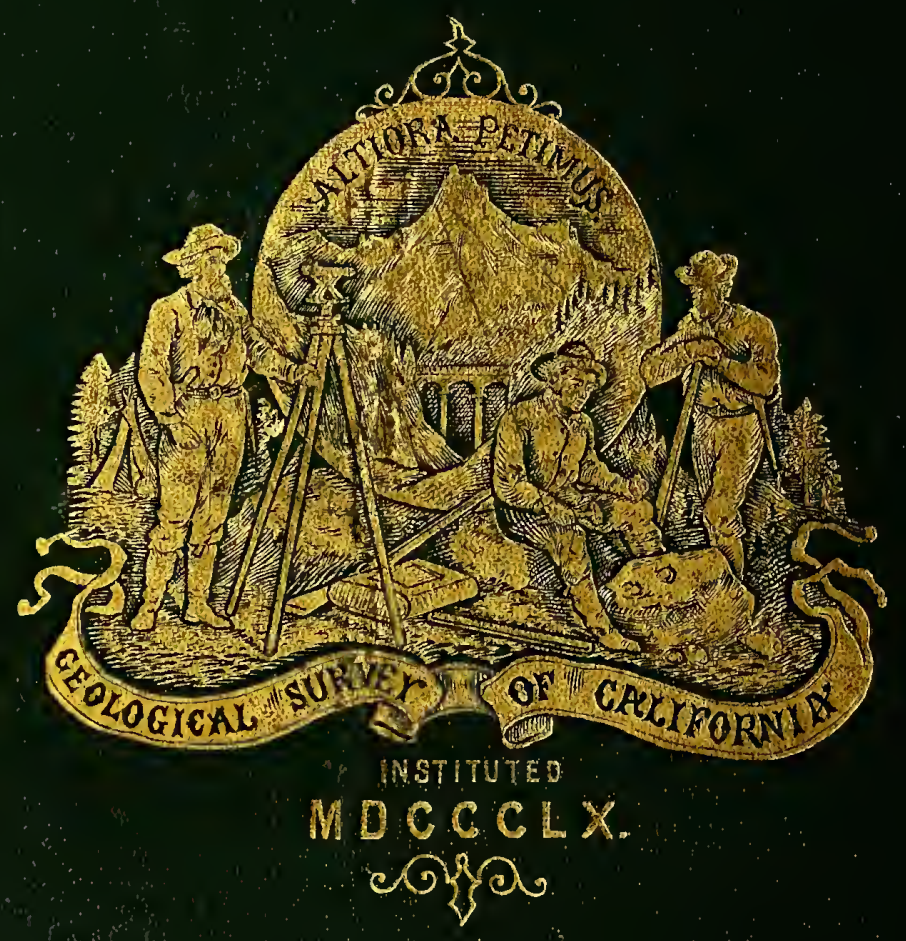

